SANDIA REPORT

SAND96-1491

Unlimited Release

Printed September 1996
RECEI:DD

OCT 231996

OSTI

\title{
Modeling Acute Health Risks Associated with Accidental Releases of Toxic Gases
}

F. Eric Haskin, Chuanyi Ding, Kenneth J. Summa, Mary Young

Prepared by

Sandia National Laboratories

Albuquerque, New Mexico 87185 and Livermore, California 94550

for the United States Department of Energy

under Contract DE-AC04-94AL 85000

$$
\text { s.ting }
$$

Approved for public release; distribution is unlimited.

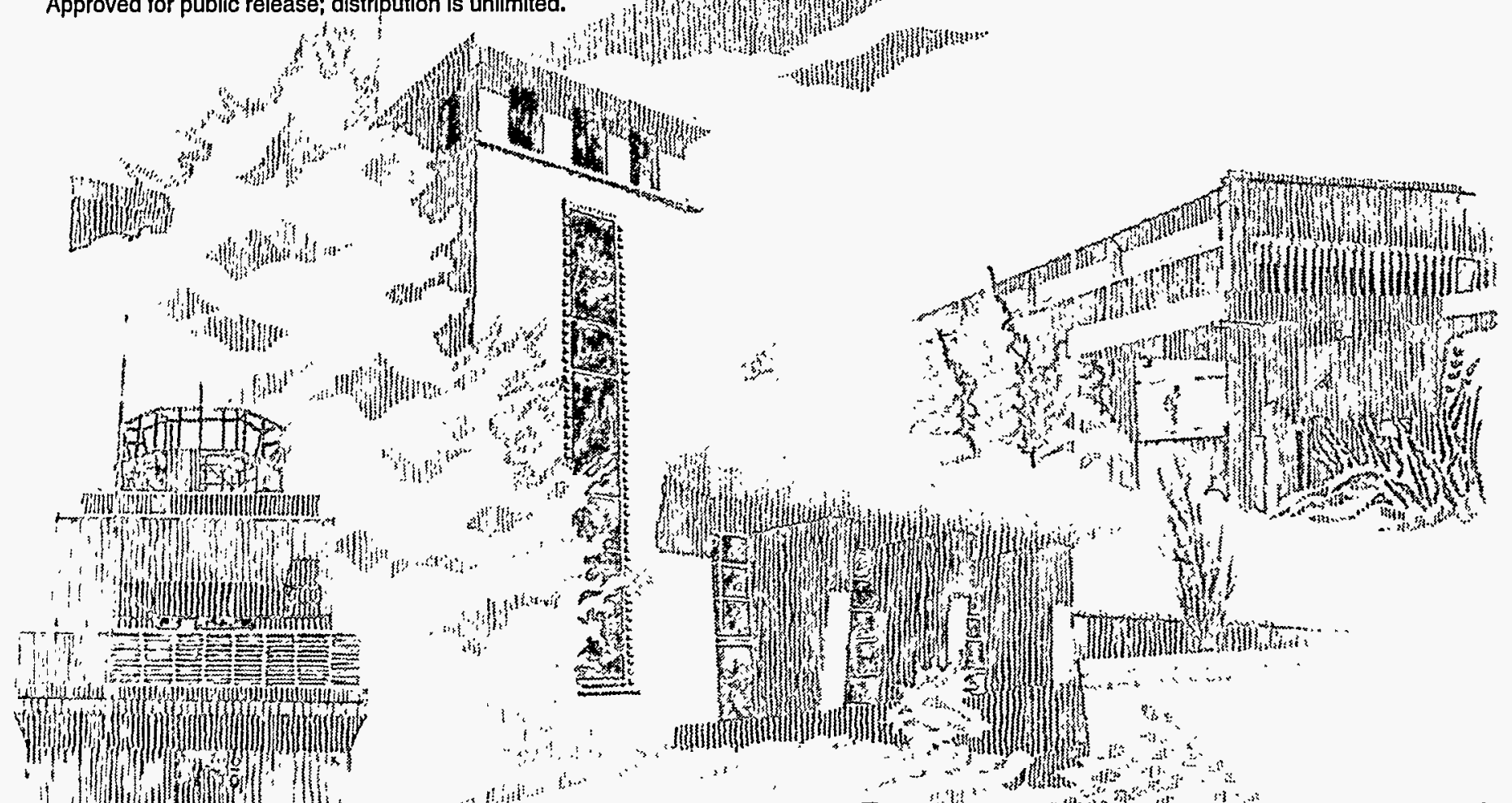
itm SF2900Q(8-81) 
Issued by Sandia National Laboratories, operated for the United States Department of Energy by Sandia Corporation.

NOTICE: This report was prepared as an account of work sponsored by an agency of the United States Government. Neither the United States Government nor any agency thereof, nor any of their employees, nor any of their contractors, subcontractors, or their employees, makes any warranty, express or implied, or assumes any legal liablity or responsibility for the accuracy, completeness, or usefulness of any information, apparatus, product, or process disclosed, or represents that its use would not infringe privately owned rights. Reference herein to any specific commercial product, process, or service by trade name, trademark, manufacturer, or otherwise, does not necessarily constitute or imply its endorsement, recommendation, or favoring by the United States Government, any agency thereof or any of their contractors or subcontractors. The views and opinions expressed herein do not necessarily state or reflect those of the United States Government, any agency thereof or any of their contractors.

Printed in the United States of America. This report has been reproduced directly from the best available copy.

Available to DOE and DOE contractors from Office of Scientific and Technical Information PO Box 62

Oak Ridge, TN 37831

Prices available from (615) 576-8401, FTS 626-8401

Available to the public from

National Technical Information Service

US Department of Commerce

5285 Port Royal RD

Springfield, VA 22161

NTIS price codes

Printed copy: A11

Microfiche copy: A01 
SAND96-1491

Unlimited Release

Printed September 1996

\title{
MODELING ACUTE HEALTH RISKS ASSOCIATED WITH ACCIDENTAL RELEASES OF TOXIC GASES
}

\author{
F. Eric Haskin, Chuanyi Ding, and Kenneth J. Summa \\ Department of Chemical and Nuclear Engineering \\ University of New Mexico \\ Albuquerque, NM 87131 \\ Mary Young \\ Accident Analysis and Consequence Assessment Department \\ Sandia National Laboratories \\ Albuquerque, NM 87185-0748
}

\begin{abstract}
CHEM_MACCS has been developed from the radiological accident consequence code, MACCS, to perform probabilistic calculations of potential off-site consequences of the accidental atmospheric release of hazardous chemicals. The principal phenomena considered in CHEM_MACCS are atmospheric transport, mitigative actions based on dose projection, dose accumulation by a number of pathways, and early and latent health effects. CHEM_MACCS provides the following capabilities: (1) statistical weather sampling data (8760 hourly data points per year), (2) population dose and health effect risk calculations based on site-specific population data, (3) health effects calculations including the consideration of potential site specific mitigative actions (evacuation and shielding), and (4) modeling of multiple release segments. Three different sample problems are contained in this report to show how to use CHEM_MACCS. Three test problems are run to compare CHEM_MACCS and D2PC. The doses versus the downwind centerline distances from the source for the given doses are in very close agreement.
\end{abstract}




\section{DISCLAIMER}

Portions of this document may be illegible in electronic image products. Images are produced from the best available original document. 



\section{CONTENTS}

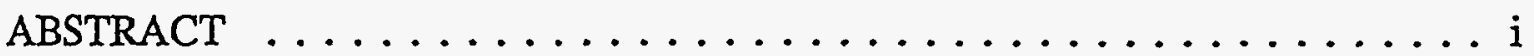

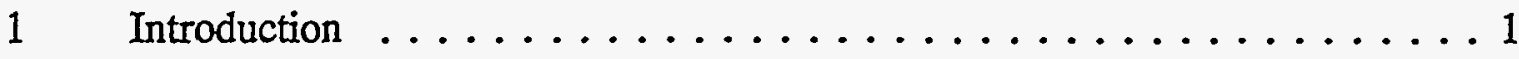

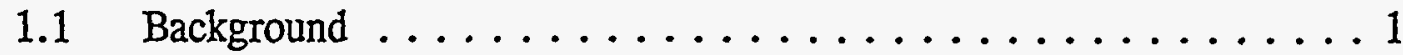

1.2 Model Overview ...................... 2

1.2.1 Input Data and Quantities Calculated by CHEM_MACCS . . . 3

1.2.2 Atmospheric Transport . . . . . . . . . . . . 4

1.2.3 Deposition, Weathering, Resuspension, and Decay .....5 5

1.2 .4 Weather Data .................. 5

1.2 .5 Dose . . . . . . . . . . . . . . . 5

1.2.6 Health Effects . . . . . . . . . . . 6

1.3 User's Overview . . . . . . . . . . . . . . 6

1.3.1 CHEM_MACCS Structure $\ldots \ldots \ldots \ldots \ldots \ldots$

2 Atmospheric Dispersion and Transport $\ldots \ldots \ldots \ldots \ldots \ldots$

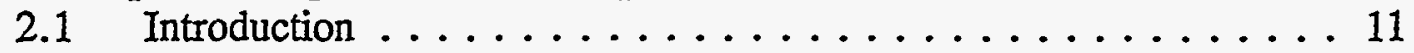

2.2 Release Specification $\ldots \ldots \ldots \ldots \ldots \ldots \ldots \ldots \ldots$

2.3 Weather Data ...................... 11

2.3.1 Weather Sequence Selection . . . . . . . . . 12

2.3.2 Weather Sequence Categorization . . . . . . . . 12

2.3 .3 Boundary Weather . . . . . . . . . . . . 12

2.4 Risk-Dominant Plume . . . . . . . . . . . . . . 13

2.5 Initial Plume Dimensions . . . . . . . . . . . . 13

2.6 Representative Weather Point . . . . . . . . . . . 14

2.7 Downwind Transport $\ldots \ldots \ldots \ldots \ldots \ldots \ldots \ldots \ldots \ldots$

2.8 Plume Rise . . . . . . . . . . . . . . 16

2.8.1 Liftoff Criterion ................. 16

2.8 .2 Plume Rise Equations . . . . . . . . . . . . . 16

2.8 .3 Mixing Height $\ldots \ldots \ldots \ldots \ldots \ldots \ldots \ldots \ldots$

2.9 Dispersion . . . . . . . . . . . . . . . . . . 19

2.9.1 Gaussian Plume Equations . . . . . . . . . . . . 19

2.9.2 Dispersion Parameters . . . . . . . . . . . 21

2.9.3 Surface Roughness . . . . . . . . . . . . . 24

2.9 .4 Plume Meander . . . . . . . . . . . . . 25

2.10 Plume Depletion . . . . . . . . . . . . . 26

$2.10 .1 \quad$ Dry Deposition . . . . . . . . . . . 26

$2.10 .2 \quad$ Wet Deposition . . . . . . . . . . . . 29

2.11 Centerline Air and Ground Concentrations .......... 31

2.12 Results Calculated by ATMOS ............. 33

3 Exposure Pathways . . . . . . . . . . . . . . . 35

3.1 Off-Centerline Correction Factors . . . . . . . . . . 35

iii 
3.2 Acute Exposure Pathways and Doses . . . . . . . . . 38

3.2.1 Plume Vapor Inhalation (PVIN) Pathway . . . . . . 38

3.2.2 Plume Vapor Skin (PVSK) Pathway . . . . . . . . . . . 39

3.2.3 Liquid-Skin (LSK) Pathway . . . . . . . . . . . . . . 40

3.2.4 Resuspended Vapor Inhalation (RVIN) Pathway . . . . . . 40

3.2.5 Resuspended Vapor Skin (RVSK) Pathway ... . . . . . 41

3.3 Cancer-Related Doses . . . . . . . . . . . . . . . . . . 41

3.3.1 Continuous Daily Dose for Plume Inhalation Pathway . . . . 41

3.3.2 Continuous Daily Dose for Resuspended Vapor Inhalation . . 42

4 Mitigative Actions and Dose Accumulation $\ldots \ldots \ldots \ldots \ldots$

4.1 Evacuation . . . . . . . . . . . . . . . 44

4.2 Population Sheltering $\ldots \ldots \ldots \ldots \ldots \ldots \ldots \ldots \ldots$

4.3 Dose Accumulation of Emergency Phase $\ldots \ldots \ldots \ldots \ldots$

$5 \quad$ Risks of Health Effects . . . . . . . . . . . . . . . . . . 49

5.1 Risks of Early Health Effects . . . . . . . . . . . . . 49

5.2 Combining Acute Doses from Different Chemicals ....... 50

5.3 Latent Cancer Risks . . . . . . . . . . . . . . 52

6 Comparison of CHEM MACCS and D2PC Results $\ldots \ldots \ldots \ldots 3$

6.1 CHEM MACCS Input $\ldots \ldots \ldots \ldots \ldots \ldots \ldots \ldots$

6.1.1 Dispersion Equations and Constants . . . . . . . 53

6.1 .2 Plume Meander Data . . . . . . . . . . . . . 55

6.1 .3 Release Description Data . . . . . . . . . . . . 55

6.1 .4 Meteorological Data . . . . . . . . . . . 55

6.2 D2PC Input Data . . . . . . . . . . . . . 55

6.3 Results ..................... 56

$7 \quad$ Summary and Recommendations . . . . . . . . . . . 59

7.1 MACCS and CHEM_MACCS .............. 59

7.2 CHEM MACCS Models . . . . . . . . . . . . . . . 59

$7: 3$ CHEM_MACCS Input and Output $\ldots \ldots \ldots \ldots \ldots \ldots$

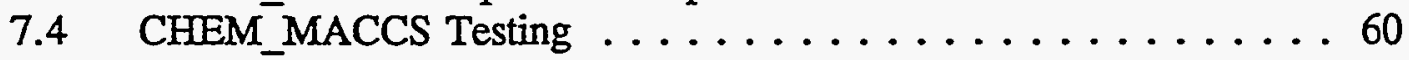

7.5 Recommendations $\ldots \ldots \ldots \ldots \ldots \ldots \ldots \ldots \ldots \ldots \ldots \ldots$

REFERENCES .......................63

APPENDIX A: User Input Processing $\ldots \ldots \ldots \ldots \ldots \ldots \ldots \ldots \ldots$ A-1

APPENDIX B: ATMOS Input File $\ldots \ldots \ldots \ldots \ldots \ldots \ldots \ldots$ B-1

APPENDIX C: EARLY Input File $\ldots \ldots \ldots \ldots \ldots \ldots \ldots \ldots$ C-1 
APPENDIX D: Sample Problem VX_A .............. D-1 APPENDIX E: CHEM_MACCS and D2PC.Comparison Runs ........ E-1 APPENDIX F: CHEM_MACCS Developmental Verification Efforts $\ldots \ldots \ldots$ F-1 APPENDIX G: Data for Probit Equations $\ldots \ldots \ldots \ldots \ldots \ldots \ldots$ G-1 


\section{FIGURES}

Figure 1 An example of conditional early fatality CCDF $\ldots \ldots \ldots$

Figure 2 Dependence of $\sigma_{y}$ and $\sigma_{z}$ on distance for the six

Pasquill-Gifford stability classes A through F . . . . . . . 22

Figure 3 Growth of $\sigma_{\mathrm{y}}$ during three time periods characterized

by different atmospheric stabilities $i, j$, and $k \ldots \ldots \ldots 24$

Figure 4 Temporal dependence of a portion of the plume's length that is over a spatial element ............... 30

Figure 5 Top-hat approximation of the Gaussian crosswind

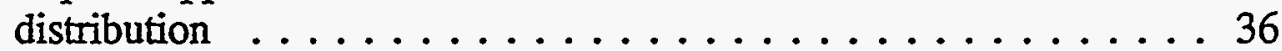

Figure 6 Approximation of a Gaussian distribution by a sevenstep histogram $\ldots \ldots \ldots \ldots \ldots \ldots \ldots \ldots \ldots \ldots \ldots$

Figure $7 \quad$ Schematic of emergency response zones $\ldots \ldots \ldots \ldots \ldots$ 


\section{TABLES}

Table 1 Estimates of the exponent $\mathrm{p}$ in Equation (2.9) for six stability classes and two surface roughnesses $\ldots \ldots \ldots \ldots 18$

Table 2 Values for constants for $\sigma_{y}$ and $\sigma_{z}$ equations $\ldots \ldots \ldots 22$

Table 3 Approximate surface roughness lengths $\left(z_{0}\right)$ for different surfaces . . . . . . . . . . . . . . . 24

Table 4 Recommended values for D2PC Sigmas . . . . . . . . 54

Table 5 Constants for CHEM-MACCS to obtain D2PC-equivalent

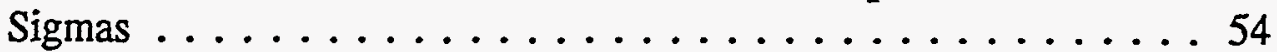

Table 6 Comparison of CHEM-MACCS and D2PC results for stability class A . . . . . . . . . . . . 56

Table 7 Comparison of CHEM-MACCS and D2PC results for stability class $\mathrm{D} \ldots \ldots \ldots \ldots \ldots \ldots \ldots \ldots \ldots \ldots$

Table 8 Comparison of CHEM-MACCS and D2PC results for stability class $\mathrm{E} \ldots \ldots \ldots \ldots \ldots \ldots \ldots \ldots \ldots$

Table F-1 CHEM_MACCS versus hand-calculated plume vapor inhalation doses . . . . . . . . . . . . . . . F-3

Table F-2 CHEM_MACCS versus hand-calculated plume vapor skin doses $\ldots \ldots \ldots \ldots \ldots \ldots \ldots \ldots \ldots \ldots$ F-4

Table F-3 CHEM_MACCS versus hand-calculated plume liquidskin doses $\ldots \ldots \ldots \ldots \ldots \ldots \ldots \ldots \ldots$ F-4

Table F-4 CHEM_MACCS versus hand-calculated resuspended plume vapor inhalation doses . . . . . . . . . . . . F F-5

Table F-5 CHEM_MACCS versus hand-calculated resuspended plume vapor skin doses $\ldots \ldots \ldots \ldots \ldots \ldots \ldots \ldots \ldots$ F-5

Table F-6 CHEM_MACCS versus hand-calculated continuous daily dose for plume vapor inhalation doses $\ldots \ldots \ldots \ldots \ldots$ F-6

Table F-7 CHEM_MACCS versus hand-calculated continuous daily dose for resuspended vapor inhalation doses $\ldots \ldots \ldots \ldots$ F-6 
Table G-1 Probit constants for CHEM_MACCS . . . . . . . . . G-3

Table G-2 DOSDATA.INP file for CHEM_MACCS . . . . . . . . G G-4 


\section{Introduction}

\subsection{Background}

On December 3, 1994, the worst industrial accident ever recorded occurred with the release of toxic gas from a Union Carbide plant in Bhopal, India. Touched off by a chemical explosion, as much as 40 tons of methyl isocyanide were released, killing 3,849 people and injuring 20,000. Union Carbide paid $\$ 470$ million to the victims and their families. Other top-ranking accidents involving deadly hazardous materials include an explosion and oil spill that resulted in 3,000 deaths at Mindoro, Philippines, in 1987; an explosion and carbon monoxide release that resulted in 2,700 deaths at Salang Pass, Afghanistan, in 1982; and an ammonium nitrate explosion that resulted in 576 deaths at Texas City, Texas, in $1947 .^{1}$

The average number of toxic chemical accidents in the United States is 19 per year. About $75 \%$ of these accidents occur at industrial facilities, while the remaining $25 \%$ result from transportation accidents. Approximately $51 \%$ of chemical accidents result from equipment failures, and about $21 \%$ are caused by human errors. Roughly one in sixteen chemical spills causes immediate injury. Fifteen percent of U.S. chemical accidents pollute surface waters; $38 \%$ pollute air and $47 \%$ pollute the land. ${ }^{1}$

Considering the high frequency and potential consequences of accidental releases of hazardous chemicals, this study was undertaken to demonstrate that methods developed to analyze accidental releases of radioactive materials from nuclear power plants can be adapted to analyze accidental releases of hazardous chemicals. The computer code adapted here is the MELCOR Accident Consequence Code System (MACCS). ${ }^{2,3,4,5}$ MACCS is used to perform probabilistic calculations of the potential offsite consequences of atmospheric releases of radioactive material in nuclear reactor accidents. MACCS was developed at Sandia National Laboratories (SNL) for the U.S. Nuclear Regulatory Commission (NRC). MACCS calculations are based on user-input, site-specific data on site weather, the population distribution surrounding the site, evacuation scenarios, and other relevant site characteristics. One year of hourly meteorological data may be input to produce a probability distribution of consequences based on the uncertainty in predicting the weather at the time of an accident.

MACCS was extensively checked and tested during its development. Since its initial release in 1990, MACCS has been continuously maintained by Sandia National Laboratories under contract with the NRC. Maintenance activities have included the documentation of code errors as reported by users, the correction of code errors, and the updating of code features as requested by users and funded by the NRC. MACCS has been inspected line by line at the Idaho National Engineering Laboratory at the request of the NRC. ${ }^{6}$ The results of MACCS calculations have been benchmarked against European, Japanese, and U.S. nuclear accident consequence codes and found to be in good agreement. ${ }^{7,8,9}$ MACCS has been used in many probabilistic risk assessments, including the NUREG-1150 study, ${ }^{10}$ and it is widely used by Department of Energy facilities for safety analysis reports. 
Science Applications International Corporation (SAIC) sponsored work by SNL and the University of New Mexico (UNM) to modify MACCS to include the capability of calculating the probabilistic offsite consequences of the accidental atmospheric release of hazardous chemicals. The version of MACCS modified to calculate the consequences of toxicological accidents is referred to as CHEM_MACCS.

This study is intended to serve as interim documentation of the CHEM_MACCS code. To accomplish this, much of the material in Chapters 1 and 2, as well as Appendices A through C, has been adapted to CHEM_MACCS with only minor changes from MACCS documentation. ${ }^{2,3}$ The other chapters and appendices are original and specific to CHEM_MACCS.

In developing CHEM_MACCS from MACCS, 58 subroutines associated with long-term exposures (the CHRONC module) and with radioactive decay were deleted, and the residual code was then modified to:

a. calculate acute doses associated with six chemical exposure pathways (Chapter 3),

b. introduce probit equations to calculate the risks of acute health effects, and Q-factors to calculate latent cancer risks (Chapter 5),

c. accept a modified DOSDATA.INP file containing probit parameters for three nerve agents (GA, GB, and VX) and the blistering agent $\mathrm{HD}$ (mustard gas)

d. add two new output options, the maximum distance at which a user-specified level of risk from early injuries is exceeded and the area of land contaminated in excess of a user-specified level,

e. allow the user to restrict the accident initiation time to normal working hours (or any other user-specified daily interval),

Verification was undertaken at each stage of the development to ensure proper implementation of these modifications (Chapter 6 and Appendix F).

\subsection{Model Overview}

Should an accidental release of toxic chemicals occur, the gases and aerosols in the plume, while dispersing in the atmosphere, would be transported by the prevailing wind. The environment would be contaminated by chemicals deposited from the plume and the public could be exposed to those chemicals. Estimating the number of health effects that could result from releases of buoyant plumes of toxic chemicals to the atmosphere is the objective of CHEM_MACCS calculations.

CHEM_MACCS is divided into two parts: ATMOS and EARLY. ATMOS treats the atmospheric transport and dispersion of toxic chemicals and their deposition onto the ground. EARLY models the effect of the release on the surrounding area during an emergency action period, which starts immediately after the release and can last up to 1 week. In this period, the exposure of the population to toxic clouds is modeled. Various protective measures can be specified for this phase, including evacuation, sheltering, and relocation. 
The probabilities of health effects in CHEM_MACCS are based on probit equations for acute health effects and potency factors for latent health effects (excess cancers) caused by exposure to released chemicals. CHEM_MACCS does not model health effects resulting from the ingestion of contaminated food or water. CHEM_MACCS models only chemical releases; it does not model radiological releases.

The polar grid used by CHEM_MACCS is centered on the release location ( $r=0$, $\theta=0$ ). The user specifies the number of radial divisions as well as their endpoint distances. Up to 35 of these divisions can be defined, extending out to a maximum distance of $9,999 \mathrm{~km}$. The angular divisions, $\theta$, of the spatial grid correspond to the 16 points of the compass. In the United States, these compass points are commonly used to express wind direction. All of the calculations of CHEM_MACCS are stored on the basis of this polar coordinate spatial grid.

Since the dose-response models for early fatalities and early injuries are highly nonlinear, some CHEM_MACCS calculations must be performed on a finer grid. For this reason, the 16 compass sectors can be subdivided into either 3,5 , or 7 userspecified subdivisions.

\subsubsection{Input Data and Quantities Calculated by CHEM_MACCS}

CHEM_MACCS calculations require the following input data:

The inventory at accident initiation of those chemicals important for the calculation of consequences;

The atmospheric source term produced by the accident (number of plume segments released; sensible heat content; timing, duration, and height of each plume segment; time when offsite officials are warned that an emergency response should be initiated; and for each important chemical, the fraction of the inventory released with each plume segment);

The population distribution around the site (distributions are constructed from census data on a polar coordinate grid having 16 angular sectors aligned with the 16 compass directions and some number of radial intervals that extend outward to $80 \mathrm{~km}$ or more);

Emergency response assumptions for evacuation (delay time of evacuation, area evacuated, average evacuation speed, and travel distance), sheltering (area sheltered), and postaccident relocation (dose criteria and relocation time);

Meteorological data characteristic of the site region (usually 1 year of hourly windspeed, atmospheric stability, and rainfall recorded at the site or at a nearby National Weather Service station). 
Given the preceding input data, CHEM_MACCS estimates the following:

The downwind transport, dispersion, and deposition of the chemicals released to the atmosphere from the facility.

The short-term doses received by exposed populations via direct pathways (skin contact and inhalation).

The mitigation of these doses by emergency.response actions (evacuation and sheltering).

The early fatalities and injuries expected to occur within 1 year of the accident (early health effects) and the delayed (latent) cancer incidences expected to occur over the lifetime of the exposed individuals.

\subsubsection{Atmospheric Transport}

CHEM MACCS allows a release of chemicals to the atmosphere to be divided into successive plume segments, which can have different compositions, release times, durations, and energies (amounts of sensible heat). Plume segment lengths are determined by the product of the segment's release duration and the average windspeed during release. The initial vertical and horizontal dimensions of each plume segment are user specified. If a release occurs into the wake of a building, then wake dimensions can be used to set the initial crosswind dimensions of the plume. If not, a point source can be specified.

A liftoff criterion (a critical windspeed that increases as plume buoyancy increases) determines whether buoyant plumes are subject to plume rise. ${ }^{11}$ When the windspeed at release equals or exceeds the critical windspeed, plume rise is prevented. When the windspeed at release is less than the critical windspeed, plume rise is allowed, and the height to which a buoyant plume rises is determined using equations recommended by Briggs. ${ }^{12,13}$

After release, windspeed determines the rates at which plume segments move in the downwind direction, and the wind direction at the time of release determines the direction of travel. As is done in many consequence codes, ${ }^{14,15}$ CHEM_MACCS neglects wind trajectories. The population distributions over the 16 compass sectors are assumed to constitute a representative set of exposed populations downwind. The exposure probability of each of the 16 population distributions is assumed to be given by the frequency with which wind blows from the site into the sector (i.e., site compass sector wind-rose frequencies).

Dispersion of the plume in the vertical and horizontal (crosswind) directions is estimated by using an empirical straight-line Gaussian plume model. ${ }^{16}$ Thus, dispersion rates depend on windspeed and atmospheric stability. Although horizontal dispersion of plume segments is unconstrained, vertical dispersion is bounded by the ground and by the top of the mixing layer (as specified by annual or seasonal mixing layer heights ${ }^{17}$ ), which are modeled as totally reflecting layers using mirror image sources. ${ }^{16}$ Since the 
number of reflections increases as travel times lengthen, eventually the vertical distribution of each plume segment becomes uniform and is so modeled thereafter. ${ }^{18}$

\subsubsection{Deposition, Weathering, Resuspension, and Decay}

In CHEM MACCS, aerosols are removed from the plume by washout, which varies with rainfall rate, ${ }^{19}$ and by diffusion to, impaction on, and gravitational settling onto surfaces. The combined removal rate from diffusion, impaction, and settling is modeled using an empirical dry deposition velocity. ${ }^{20}$ Because the dry deposition velocity varies with particle size, if the aerosol size distribution is divided into ranges, a dry-deposition velocity must be specified for each range.

Weathering, resuspension, and washoff decrease surface concentrations of chemicals deposited on the ground. Weathering is modeled using Gale's equation. ${ }^{21}$ Resuspension is modeled using resuspension factors that attempt to represent the average effect of resuspension by many processes at very different rates throughout large regions. ${ }^{20}$. Washoff is modeled as a first-order removal process that is integrated over all time after the initial deposition. ${ }^{22}$

\subsubsection{Weather Data}

Plume rise, dispersion, downwind transport, and deposition depend on prevailing weather conditions (windspeed, atmospheric stability, rain rate). In CHEM_MACCS these may either be invariant or may vary hour by hour. If variable, they can be user specified or can be read from a weather file. When variable weather data are used to model a multiple segment release, one of the plume segments must be specified as risk dominant. Usually, the risk-dominant segment will be the segment that produces the acute doses that dominate early fatalities. Once a risk-dominant segment has been specified, CHEM_MACCS automatically causes the leading edge of that segment to be released at the beginning of the first hour of weather data in the hourly sequence of variable weather data.

\subsubsection{Dose}

The CHEM_MACCS dose model consists of three interacting processes: projection of individual exposures for each of the exposure pathways modeled, mitigation of these exposures by protective measures, and calculation of the actual exposures incurred after mitigation by protective measures. For each exposure pathway, CHEM_MACCS models the reduced burden that results from mitigating actions.

\subsubsection{Dose Mitigation}

During the emergency phase, which can last up to 7 days, exposure is reduced by evacuation, sheltering, and temporary relocation of people. In CHEM MACCS, people relocate only if their projected doses exceed a user-specified limit. In contrast to temporary relocation, evacuation and sheltering automatically take place within some specified region without regard to projected exposures. After a delay period that follows 
an optional period of sheltering, additional exposure can be incurred during evacuation of the populace. Evacuation is assumed to be radially outward. The speed of evacuation can be varied to account for the possibility of traffic jams and impeded travel. At a userspecified distance from the facility, evacuees are assumed to be directed out of the path of the plume so that further exposures are avoided.

\subsubsection{Exposure Pathways}

The chemical doses calculated in CHEM_MACCS are closely associated with specific exposure pathways. The five different pathways discussed in Section 3.2 are inhalation of plume vapor, skin contact with plume vapor, skin contact with plume liquid, inhalation of resuspended vapor, and skin contact with resuspended vapor.

\subsubsection{Protection Factors}

CHEM_MACCS assigns people to three groups: evacuees, people actively taking shelter, and people who continue normal activities. Doses to evacuees are calculated using vehicle protection factors. Protection factors for people who actively take shelter are smaller (i.e., they are better protected) than for people who continue normal indoor activities: This is because people who actively take shelter are assumed to close doors and windows and turn off air circulation systems. Protection factors for people are calculated outside of the code and specified by the user.

\subsubsection{Health Effects}

The calculations of health effects in CHEM_MACCS are based on probit equations for acute health effects and potency factors for the calculation of latent health effects (excess cancers) caused by exposure to released chemicals. CHEM_MACCS does not model health effects resulting from the ingestion of contaminated food or water. CHEM_MACCS models only chemical releases; it does not model radiological releases.

Most of the data relating chemical doses and specific health effects are for exposures to only one chemical species. The CHEM_MACCS method of combining doses from different chemicals is to linearly weight the relevant air and skin concentrations of different chemicals in proportion to their ability to cause the health effect in question. The basis for this approach is discussed in Section 5.2.

\subsection{User's Overview}

Accidents can lead to source terms of quite different magnitudes, and the weather conditions at the time of the release can greatly alter the magnitude of consequences (e.g., intense rain at the time of the release or plume transport out to sea would largely eliminate health effects, while rainout of the plume onto a nearby city could greatly increase early health effects).

Because consequences vary with source term magnitude, weather, and population density, consequence assessments must examine all possible combinations of 
representative sets of source terms, weather sequences, and exposed populations. Usually distributions that display the variation of consequences with weather and population density are first developed for each representative source term. Then, an integral depiction of consequences may be constructed by a weighted summation of these source-term dependent distributions, with each distribution weighted by the estimated absolute probability of occurrence of its underlying source term.

Given a source term, a CHEM_MACCS consequence calculation generates results for all possible combinations of a representative set of weather sequences (usually about 150 sequences) and a representative set of exposed populations downwind (usually 16), thereby producing about 2,400 results for each consequence measure examined. Since the probability of occurrence of each weather sequence $\left(P_{w i}\right)$ and the exposure probability of each population distribution $\left(\mathrm{P}_{\mathrm{p}}\right)$ are known, the variability with weather and population of the 2,400 results can be displayed by a complementary cumulative distribution function (CCDF) of consequence magnitude. ${ }^{14}$

Figure 1 presents a CCDF of the number of early fatalities that resulted from 2,400 trials. The following statistical results may be extracted from a CCDF: the probability that any consequence occurs ( $y$-intercept); the expected (mean) consequence,

$$
E(X)=\sum_{i} P_{i} X_{i}
$$

where $P_{i}$ is the probability $\left(P_{i}=P_{w i} P_{p i}\right)$ and $X_{i}$ is the magnitude of each of the 2,400 results; the consequence magnitudes that correspond to given quantile values (e.g., for any consequence the 90th quantile is the consequence magnitude that has a conditional probability 0.1 of being equaled or exceeded); and the largest consequence magnitude calculated for any weather trial (the consequence magnitude that corresponds to the tail of the CCDF). For the example shown in Figure 1; the probability of having any early fatalities is 0.2 ; the early fatality 90 th quantile is. 2 ; and the largest result calculated is 30 .

To facilitate uncertainty and sensitivity analyses, the value of almost every parameter used in the CHEM_MACCS code can be changed by the user. The input format is self-documenting to make such changes easier to implement. Appendices A, $\mathrm{B}$, and $\mathrm{C}$ describe CHEM_MACCS input in detail.

Unless specified otherwise, all times used in CHEM_MACCS are referenced to the time of accident initiation. Throughout CHEM_MACCS, standard international units have been used to the greatest possible extent. 


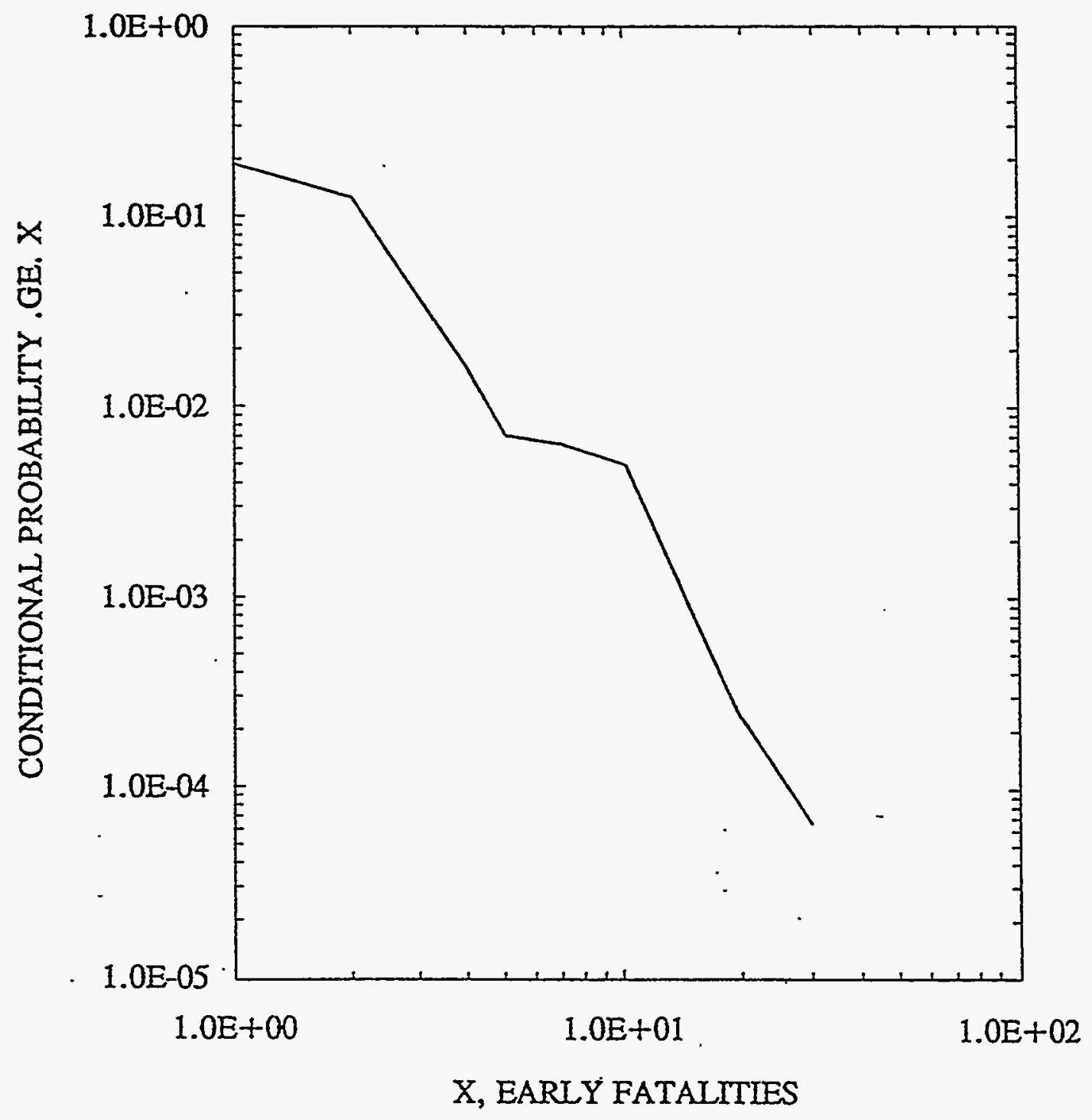

Figure 1 An example of conditional early fatality CCDF 


\subsubsection{CHEM_MACCS Structure}

CHEM_MACCS modeling calculations are preceded by processing of all input to the code. There is extensive checking for errors so that input errors are located and diagnosed before the modeling calculations are performed. If any errors are detected, the program tries to validate as much of the subsequent input as possible in order to facilitate the debugging process, but execution of the program is then terminated.

All the phenomenological modeling occurs in the second phase of a CHEM_MACCS run. The sequence in which the phenomena are evaluated closely follows the temporal order of events in the real world. The phenomenological models are for the most part based on empirical data and the solutions they entail are usually analytical in nature and computationally straightforward. There are two modules: ATMOS and EARLY.

ATMOS treats the atmospheric transport and dispersion of material and its deposition from the air. EARLY models the effect of the accident on the surrounding area during an emergency action period, which starts immediately after the accident and which can last up to 1 week.

ATMOS defines the weather conditions, initializes the dimensions of the plume; determines whether there will be any plume rise; calculates the arrival time of the plume, the amount of material remaining after wet deposition, and the dispersion of the plume. It determines the amount of plume rise of a buoyant plume; calculates atmospheric dispersion, ignoring deposition, the amount of material remaining after dry deposition, the amount of material lost from dry and wet deposition together, and average air and ground concentrations. Finally, it indicates how much of the plume is left after deposition and updates the particle size distribution.

EARLY calculates the histogram approximating the Gaussian distribution, the cloudshine correction factor, the doses in the relocation zone (nonshelterees, nonevacuees), the doses to shelters and evacuees while they are stationary, the doses to evacuees while they are moving, and the health-effect risks requested by the user.

The data needed to define these models are specified through two user input files, one for ATMOS and one for EARLY. The requirements for preparation of these files are described in Appendices B and C. 
This page intentionally left blank. 


\section{Atmospheric Dispersion and Transport}

\subsection{Introduction}

ATMOS treats downwind transport, dispersion, and deposition. In addition to the values of the parameters implemented in the phenomenological models, the ATMOS module also requires that the nature of the release and the dimensions of the computational grid be specified as input. Given these data, ATMOS then models plume liftoff and rise, the capping of plume rise and of vertical plume expansion by inversion layers, downwind transport of the plume, dry horizontal and vertical dispersion of the plume, plume depletion by wet and dry deposition, and the centerline air and ground concentrations that these processes produce on the computational grid.

Most of the models implemented in ATMOS use weather conditions as input data. Either constant or variable weather data can be used. Variable data are specified as a sequence of hourly values of windspeed, class of atmospheric stability, and amount of precipitation that begins at a time specified by the user or selected by the weather categorization and sampling algorithm embedded in the code. If variable data are used to model a release that is divided into plume segments, the user must designate one of the segments as risk dominant, whereupon ATMOS automatically causes the release of that segment to coincide with the start time of (first hour of data in) the variable sequence of weather conditions. The user must also select a representative weather bin point for each plume segment, which determines the weather conditions that will be used to calculate all transport processes except wet deposition. The phenomenological models implemented in the ATMOS module of CHEM_MACCS are described in the following sections of this chapter.

\subsection{Release Specification}

To model long-duration releases, CHEM MACCS allows any plume to be divided into segments. The amount of material released in each plume segment is specified by a set of release fractions. One release fraction, referenced to the inventory of the facility at the time of the postulated accident, must be specified for each chemical species. In addition to the release fractions, CHEM_MACCS also requires that the release time and duration, warning time before release, the release height, and the sensible heat content of each plume segment be specified.

\subsection{Weather Data}

The atmospheric transport models implemented in CHEM_MACCS require hourly readings of windspeed, wind direction, atmospheric stability, and rainfall (precipitation) as input. For each weather sequence examined by ATMOS, $120 \mathrm{hr}$ of weather data are required. In addition, four values of the mixing height (height of the capping inversion layer), one for each season of the year, must also be specified. 


\subsubsection{Weather Sequence Selection}

In CHEM_MACCS, there are five ways to specify the required $120 \mathrm{hr}$ of weather data that constitute a weather sequence:

Constant Weather Conditions: one windspeed, one atmospheric stability class, one hourly amount of precipitation.

User-specified Weather Sequence: 120 hourly readings of windspeed, stability class, and precipitation amounts.

User-specified Start Time: the day and hour in a 1-year-long weather file when the 120-hr-long weather sequence selected for use begins.

Stratified Random Sampling: the length of the time period (number of hours or days) from which one start time is to be selected by random sampling from a 1-year-long weather file (e.g., randomly select one start time from each sequential period of $12 \mathrm{hr}$ ).

Structured Monte-Carlo Sampling: random selection of a user-specified number of weather sequences (start times) from the set of sequences assigned to user-specified weather categories by sorting a 1 year-long weather file according to user-specified criteria (a 1 year-long file of hourly weather data contains $8,760 \mathrm{hr}$ of data and thus 8,760 sequence start times).

\subsubsection{Weather Sequence Categorization}

The algorithm used by CHEM_MACCS to categorize weather sequences (start times) is similar to the algorithm implemented in an earlier consequence modeling code (CRAC2). ${ }^{23}$ The algorithm sorts weather sequence start times (start day and hour) into weather categories in two ways:

Initial Conditions: stability class and windspeed during the start hour of the sequence (e.g., F-stability with a windspeed between 1 and $2 \mathrm{~m} / \mathrm{s}$ ).

Precipitation Characteristics: the downwind distance at which precipitation begins and the rate $(\mathrm{mm} / \mathrm{hr})$ of that precipitation (e.g., precipitation will start after the plume segment has moved $3 \mathrm{~km}$ and before it has moved $6 \mathrm{~km}$; the precipitation rate during the first hour in which precipitation occurs will lie between 1 and $2 \mathrm{~mm} / \mathrm{hr}$ ).

\subsubsection{Boundary Weather}

Because $120 \mathrm{hr}$ of weather data may not be sufficient to transport a single segment release (or the last plume segment in a multiple segment release) off the computational grid, a constant set of weather conditions called "boundary weather" must be specified 
for use should a segment still be partly or wholly on the computational grid after the last hour of data in the 120-hr weather sequence has been used. The CHEM_MACCS user may also specify that boundary weather will be used whenever a plume segment reaches a user-specified downwind distance (spatial element on the computational grid).

Boundary weather is required by ATMOS to model (1) short releases that encounter persistent, low windspeed conditions and (2) releases of long duration. Two examples illustrate the need for boundary weather. First, a 1-hr release will require at least $125 \mathrm{hr}$ of data to traverse a 500-mile computational grid if all of the hourly windspeed readings in the $120 \mathrm{hr}$ of data lie below $4 \mathrm{mph}$. Second, even if average windspeed in the 120-hr sequence is as high as $5 \mathrm{mph}$, a release composed of a blowdown puff and a lengthy ( $20 \mathrm{hr}$ ) tail will also require more than $120 \mathrm{hr}$ of weather data to ensure transport of the tail across the entire 500-mile computational grid.

Finally, the CHEM_MACCS user can use boundary weather to ensure that all of the aerosols in a plume segment are deposited on the ground before the segment completely traverses the computational grid. This is done either by specifying boundary weather with a very low windspeed (the very slow transport rate allows dry deposition to completely deplete the plume segment of aerosols) or by specifying a high rain rate (whereupon all aerosols are removed by washout).

\subsection{Risk-Dominant Plume}

When variable weather data are used to model a multiple segment release, one of the plume segments must be designated as the risk-dominant segment. The characteristics of the categorization algorithm used to sort weather sequences will usually suggest how the risk-dominant segment should be chosen. For CHEM MACCS, weather sequences can be sorted according to their potential to cause early fatalities (e.g., sorted for stable atmospheric conditions upon release and for rainfall within 20 miles of the site), and the plume segment expected to make the largest contribution to the induction of early fatalities is designated as the risk-dominant segment: Once a risk-dominant segment has been designated, CHEM_MACCS automatically causes the leading edge of that segment to be released at the start time (beginning of the first hour) of each weather sequence used in the calculation. If the source term contains plume segments released earlier than the designated risk-dominant segment (e.g., a leak that precedes a large blowdown puff), then hourly data that precede the start hour of the selected sequence are used to govern the transport of the earlier segments.

\subsection{Initial Plume Dimensions}

As discussed in Section 2.9, CHEM_MACCS models plume dispersion during downwind transport using Gaussian plume models. Thus, the horizontal and vertical extent of plume segments is expressed in terms of the horizontal $\left(\sigma_{y}\right)$ and vertical $\left(\sigma_{2}\right)$ standard deviations of the normal concentration distributions that characterize a Gaussian plume. The Gaussian equations implemented in CHEM_MACCS are derived assuming that turbulent velocities are negligible compared with the mean windspeed..$^{16}$ Accordingly, CHEM_MACCS assumes that the initial length of plume segments is 
unaffected by diffusion during downwind transport (i.e., plume segment lengths are constant once release from containment is completed). Thus, plume segment lengths, $\mathrm{L}$ (in meters), are given by:

$$
L=\sum_{i}\left(\Delta t_{i} \cdot v_{i}\right)
$$

where

$$
\begin{aligned}
& \sum_{i} \Delta t_{i}=\quad \text { the release duration of the plume segment, and } \\
& t_{i}=\quad \begin{array}{l}
\text { a part of the release duration during which the windspeed } \\
\text { was } v_{i} \cdot{ }^{18}
\end{array}
\end{aligned}
$$

When release occurs under turbulent conditions, mixing of the plume in the wake of the building from which the release occurs will determine the initial crosswind dimensions of the plume. For the purpose of initializing plume dimensions, CHEM_MACCS assumes the release point to be at ground level and in the middle of the downwind face of the building. If plume concentrations at the sides and roofline of the building from which the release occurs are assumed to be 10 percent of plume centerline concentrations (building edges are 2.15 sigma from the plume centerline), then initial values of the horizontal and vertical standard deviations of the Gaussian plume are given by

$$
\begin{aligned}
& \sigma_{y}(t=0)=W_{b} / 4.3 \\
& \sigma_{z}(t=0)=H_{b} / 2.15
\end{aligned}
$$

where $W_{b}$ and $H_{b}$ are the width and height of the building wake and are specified by the user.

\subsection{Representative Weather Point}

There is a fundamental problem in treating the long-duration releases (plume segments with release times of many hours) that are allowed in CHEM_MACCS. Since the segment's characteristics must be uniform along its length, weather conditions at one point along that length must be used for all points along its length. If the weather conditions experienced by a plume's leading edge are used to control plume transport (plume rise, downwind transport, dispersion, and deposition), transport processes are modeled as though all plume materials are concentrated at the head of the plume.

For short plume segments (segments with short release times), this is a reasonable approximation. However, for segments with release times of many hours, use of weather experienced by some interior point along the length of the segment would seem to be a better representation of the average weather conditions along the entire length of the segment. 
Accordingly, the CHEM_MACCS user must select a representative weather point for each plume segment. This is a fixed point along the length of the plume (usually the segment's leading edge or midpoint). Once selected, the weather conditions experienced by that point are applied to the entire length of the plume to calculate plume rise, transport, dispersion, and dry deposition (wet deposition is calculated using the rain rates experienced hour by hour by each portion of the plume; see Appendix C.10). The representative weather point is specified as a fraction that can assume any value from zero to one, where values of $0.0,0.5$, and 1.0 correspond to selection of weather conditions experienced by the head, midpoint, and tail of the segment.

\subsection{Downwind Transport}

After a plume segment is fully released, its length does not change during downwind transport (except when a transition from weather sequence data to boundary weather occurs). The arrival time of any reference point along the plume's length (e.g., head, midpoint, tail) at any downwind grid point (e.g., near boundary, midpoint, or far boundary of some spatial element) is determined by the following equations:

$$
\begin{aligned}
& d=\sum_{i=1}^{n} v_{i} \Delta t_{i} \\
& \Delta t_{d}=\sum_{i=1}^{n} \Delta t_{i}
\end{aligned}
$$

where

$$
\begin{aligned}
& \mathrm{d}=\text { downwind (radial) distance of the grid point from the } \\
& \text { facility (center of the polar coordinate computational grid), } \\
& \Delta t_{d}=\text { arrival time of the reference point at the distance } d \text {, } \\
& v_{i}=\text { windspeed during the time period } \Delta t_{i} \text {, and } \\
& \mathrm{n}=\text {. number of time periods. }
\end{aligned}
$$

The values of $\Delta t_{2}$ through $\Delta t_{n-1}$ are all equal to $1 \mathrm{hr}$ and $\Delta \mathrm{t}_{1}$ and $\Delta \mathrm{t}_{\mathrm{n}}$ may be parts of an hour. All times are measured from the time of release of the plume segment's reference point. Thus, the time of arrival of any part of a plume segment at any downwind location is easily calculated. Finally, since the arrival time of the head $\left(t_{t}\right)$ and the tail $(\mathrm{t})$ of a plume segment at any downwind location (e.g., grid element midpoint) can be calculated, the time period $\left(\Delta \mathrm{t}_{\mathrm{r}}\right)$ that a person situated at that location is exposed to the passing plume is given by

$$
\Delta t_{c}=t_{t}-t_{h}
$$




\subsection{Plume Rise}

Plume segments that are hot (contain appreciable sensible heat) and thus buoyant may rise to heights much greater than their initial release height. In CHEM_MACCS, plume rise is calculated using equations recommended by Briggs. ${ }^{12,13}$ Plume rise is inhibited whenever the prevailing windspeed at the time of release exceeds a critical windspeed (liftoff criterion). Plume rise is also limited by the mixing height (height of any capping inversion layer).

\subsubsection{Liftoff Criterion}

When windspeeds are high, a buoyant plume segment released into a strong building wake will be unable to escape from the wake. In CHEM_MACCS, escape of buoyant plume segments from building wakes is governed by a liftoff criterion [Equation (2.5)] proposed by Briggs and validated by experiments performed at the Warren Spring Laboratory in Great Britain. ${ }^{24,11}$ The criterion states that plume rise occurs only when the windspeed upon release of the segment is less than a critical windspeed $\left(u_{c}\right)$ calculated using the following formula, ${ }^{11}$

$$
u_{c}=\left[\frac{9.09 \mathrm{~F}}{L_{p}}\right]^{1 / 3}
$$

where $L_{p}$ is a plume scale length (m) (e.g., the height of the building) and $F$ is the buoyancy flux $\left(\mathrm{m}^{4} / \mathrm{s}^{3}\right)$ of the source (plume segment), which depends both on ambient atmospheric conditions and on the sensible heat release rate (D) of the plume segment (sensible heat content of the segment divided by its release duration). When the ambient conditions used to calculate F correspond to the International Civil Aviation Organization Standard Atmosphere, ${ }^{25} \quad \mathrm{~F}=8.79 \times 10^{-6} \dot{\mathrm{Q}}$, where $\dot{\mathrm{Q}}$ is expressed in watts.

\subsubsection{Plume Rise Equations}

When atmospheric conditions are neutral or unstable (stability classes A-D), plume rise is treated using the Brigg's "two thirds" law for bent-over plumes ${ }^{13}$ :

$$
\Delta \mathrm{h}=\frac{1.6 \mathrm{~F}^{1 / 3} \mathrm{x}^{2 / 3}}{\overline{\mathrm{u}}}
$$

where

$$
\begin{aligned}
& \Delta \mathrm{h}=\text { amount of plume rise (m), } \\
& \mathrm{F}=8.79 \times 10^{-6} \mathrm{Q} \text {, the buoyancy flux }\left(\mathrm{m}^{4} / \mathrm{s}^{3}\right) \text { of the plume } \\
& \text { segment, } \\
& \dot{\mathrm{Q}}=\text { rate of release of sensible heat }(\mathrm{w}) \text {, } \\
& \mathrm{x} .=\text { downwind (radial) distance }(\mathrm{m}) \text {, and } \\
& \text { प }=\text { mean windspeed }(\mathrm{m} / \mathrm{s}) \text {. }
\end{aligned}
$$


Buoyant plume rise is terminated when any of the following conditions occur:

1. when, as is recommended by Briggs, ${ }^{12} \Delta \mathrm{h}$ reaches $300 \mathrm{~F} / \mathrm{u}^{3}$

2. when $\mathrm{H}=\mathrm{L}$, where $\mathrm{H}$ is the height of the plume centerline and $\mathrm{L}$ is the mixing height (height of the capping inversion layer)

3. when $1 \mathrm{hr}$ has elapsed since release of the plume segment began.

When Equation (2.6) is used in CHEM_MACCS, the weather conditions that characterize the hour during which release of the segment begins are used to calculate the entire rise of the segment even when the rise extends into the next hour (e.g., a buoyant plume segment released at 1:30 p.m., which rises under unstable conditions for a full hour, would have its entire rise calculated using 1:00 p.m. weather).

When atmospheric conditions are stable (stability classes $E$ and $F$ ), plume rise is calculated using the Briggs equation for the final rise $(h)$ of a bent-over buoyant plume ${ }^{13}$ :

$$
\Delta h=2.6\left[\frac{F}{\bar{u} S}\right]^{1 / 3}
$$

where

$$
\begin{aligned}
& \mathrm{S}=\text { stability parameter }\left(\mathrm{s}^{-2}\right) \text { defined by the following equation. }{ }^{13} \\
& S=\frac{g}{T_{a}}\left[\frac{\partial T_{z}}{\partial z}+\frac{g}{c_{p}}\right] \\
& \mathrm{g}=\text { acceleration due to gravity }\left(\mathrm{m} / \mathrm{s}^{2}\right) \text {, } \\
& \mathrm{T}_{\mathrm{a}}=\text { ambient temperature }(\mathrm{K}) \text {, } \\
& \partial \mathrm{T}_{\mathrm{z}} / \partial \mathrm{z}=\text {. ambient temperature lapse rate }(\mathrm{K} / \mathrm{m}) \text {, } \\
& c_{\mathrm{p}}=\text { heat capacity of air }(\mathrm{J} / \mathrm{kg}-\mathrm{K}) \text {, and } \\
& \mathrm{g} / \mathrm{c}_{\mathrm{p}}=\text { dry adiabatic lapse rate }(0.98 \mathrm{~K} / 100 \mathrm{~m}) \text {. }
\end{aligned}
$$

Regulatory Guide 1.23 specifies ranges for temperature lapse rates $\left(\partial \mathrm{T}_{\mathrm{a}} / \partial \mathrm{z}\right)$ for the six atmospheric stability classes A through $\mathrm{F}^{26}$ The values of the stability parameter $S$ used in CHEM_MACCS were derived using midpoint values for these lapse rate ranges. The lapse rate ranges specified for stability classes $E$ and $F$ are $-0.5 \mathrm{~K} / 100 \mathrm{~m}$ to $1.5 \mathrm{~K} / 100 \mathrm{~m}$ and $1.5 \mathrm{~K} / 100 \mathrm{~m}$ to $4.0 \mathrm{~K} / 100 \mathrm{~m}$, respectively. Thus, class $\mathrm{E}$ has a lapse rate midpoint of $0.5 \mathrm{~K} / 100 \mathrm{~m}$ and class $\mathrm{F}$ a midpoint of $2.75 \mathrm{~K} / 100 \mathrm{~m}$. Substitution of these midpoint values and the International Civil Aviation Organization standard atmosphere value of $288.16 \mathrm{~K}\left(15^{\circ} \mathrm{C}\right)$ into Equation (2.8) yields values of $5.04 \times 10^{-4} \mathrm{~s}^{-2}$ and $1.27 \times 10^{-3} \mathrm{~s}^{-2}$ for the stability parameter $S$ for stability classes $E$ and $F_{.}{ }^{25}$

Because near-surface windspeeds increase with altitude, Equations (2.6) and (2.7) both overestimate plume rise if surface windspeeds are used to calculate $\Delta$ h. Since this 
could produce a significant underestimation of exposures, for purposes of calculating plume rise, windspeeds aloft are estimated from surface windspeeds using the following equation ${ }^{13}$ :

$$
u=u_{0}\left(\frac{z}{z_{0}}\right]^{p}
$$

where

$$
\begin{array}{ll}
\mathrm{u}_{0}= & \text { surface windspeed }(\mathrm{m} / \mathrm{s}) \text { at the reference height } \mathrm{z}_{0} \text { (usually } 10 \mathrm{~m} \text { ), } \\
\mathrm{u}= & \text { windspeed }(\mathrm{m} / \mathrm{s}) \text { at the height } \mathrm{z} \text {, and } \\
\mathrm{p}= & \text { parameter (dimensionless) that varies with stability class and } \\
\text { surface roughness as shown in Table } 1 . .^{13}
\end{array}
$$

At present, the values of $p$ for rural surfaces are hard-wired into CHEM_MACCS. The maximum value of $z$ in Equation (2.9) is $200 \mathrm{~m}$.

Table 1 Estimates of the exponent $p$ in Equation (2.9) for six stability classes and two surface roughnesses

\begin{tabular}{l|c|c|c|c|c|c}
\hline Stability Class & A & B & C & D & E & F \\
\hline Urban surfaces & 0.15 & 0.15 & 0.20 & 0.25 & 0.40 & 0.60 \\
Rural surfaces & 0.07 & 0.07 & 0.10 & 0.15 & 0.35 & 0.55 \\
\hline
\end{tabular}

In CHEM_MACCS an average value of $u$ for use in Equations (2.6) or (2.7) is calculated in three steps. First, the surface windspeed $u_{0}$ and either Equation (2.6) or Equation (2.7) are used to make a first-order estimate of the final centerline height (z) of the plume segment after plume rise has taken place $\left(z=h_{0}+\Delta h\right.$, where $h_{0}$ is the initial release height of the plume segment). Then the windspeed $u$ at the height $z$ is calculated using Equation (2.9). Finally, an average windspeed over this range is estimated by averaging $\mathfrak{u}_{0}$, the reference windspeed, and $\mathfrak{u}$, the windspeed at the first-order estimate of the final height of the plume centerline. This average value of $u$ is then used in either Equations (2.6) or (2.7) to make a second-order estimate of the amount of plume rise, $\Delta \mathrm{h}$.

\subsubsection{Mixing Height}

A single value for the mixing height (the top of the well-mixed surface layer of air, frequently the location of the lowest-lying temperature inversion in the temperature structure of the surface layer) is used in CHEM_MACCS to limit both buoyant plume rise and the vertical dispersion (see Section 2.9) of plume segments. Although the value is allowed to vary season by season, it does not vary with stability class and is held 
constant during each weather trial (it does not change even if the weather trial begins in one season and ends in another).

Because the mixing height is used in CHEM_MACCS as an impenetrable cap, normally afternoon mixing heights should be used in CHEM_MACCS calculations. ${ }^{17}$ If the concentrating effects of low-lying inversion layers (e.g., radiation inversions) are examined, the user should remember that CHEM_MACCS models neither penetration of inversion layers by buoyant plumes, ${ }^{27}$ nor temporal variation in the height of the mixing layer (normally the depth of the well-mixed layer increases from several hundred meters at sunrise to several thousand meters by midafternoon ${ }^{17}$ ).

\subsection{Dispersion}

During downwind transport, atmospheric turbulence will cause plume segments to expand in all directions, with the rate of expansion increasing as atmospheric turbulence increases. Vertical expansion of the plume is increased by surface roughness and constrained by the ground and by the temperature structure of the atmosphere (location of inversion layers). Crosswind spreading of the plume along the y-direction is unconstrained. The effective crosswind dimensions of a plume segment are increased by lateral meander of the plume about its centerline trajectory. Because turbulent velocities are almost always very small compared with the mean wind speed that transports the bulk plume, expansion in the along-wind direction can be neglected. ${ }^{18}$

\subsubsection{Gaussian Plume Equations}

Because they are simple and computationally efficient, Gaussian plume models have often been used to model atmospheric dispersion in risk assessments (see, for example, the PRA Procedures Guide ${ }^{14}$ ). Gaussian plume models assume that the diffusion of gas molecules and aerosol particles in a plume during its downwind transport can be modeled as a random walk that generates a normal distribution for air concentration in all directions. Because windspeed and temperature vary significantly with height near the ground, vertical and horizontal plume distributions differ greatly. Because the along-wind distribution does not appear in the Gaussian plume equations implemented in CHEM_MACCS, only the vertical and crosswind distributions are actually calculated.

The size of a Gaussian plume in the vertical and crosswind directions is indicated by the standard deviations ( $\sigma_{y}$ and $\sigma_{z}$ ) of the normal distributions of material concentrations in the vertical and crosswind directions. When not constrained by the ground or by inversion layers, the Gaussian plume equation has the following form ${ }^{18}$ :

$$
x(x, y, z)=\frac{Q}{2 \pi \bar{u} \sigma_{y} \sigma_{z}} \exp \left[-\frac{1}{2}\left(\frac{y}{\sigma_{y}}\right]^{2}\right] \exp \left[-\frac{1}{2}\left[\frac{z-h}{\sigma_{z}}\right]^{2}\right]
$$

where 
$\chi(\mathrm{x}, \mathrm{y}, \mathrm{z})=$ time-integrated air concentration $\left(\mathrm{mg}-\mathrm{s} / \mathrm{m}^{3}\right)$ at the downwind location $(x, y, z)$,

$\underline{\mathrm{Q}} \quad=\quad$ source strength $(\mathrm{mg})$,

$\overline{\mathrm{u}}=$ mean windspeed $(\mathrm{m} / \mathrm{s})$,

$\sigma_{\mathrm{y}}$ and $\sigma_{\mathrm{z}}=$ standard deviations (m) of the normal crosswind and vertical concentration distributions of plume materials,

$(0,0, h)=\quad$ source location, and

$\mathrm{h}=$ initial release height $(\mathrm{m})$.

Once a plume has expanded sufficiently in the vertical direction so that further vertical expansion is constrained by the ground and/or the capping inversion layer, Equation (2.10) can no longer be used. To treat restricted growth in the vertical direction, the ground and the inversion layer are treated as impenetrable, totally reflecting boundaries. Mathematically, reflection is accomplished by the addition of mirror image sources above the inversion layer and below the plane of the ground. This produces the following equation, which is used in CHEM_MACCS to calculate both the plume centerline air concentration $(x, y=0, z=H)$ and ground-level air concentration under the plume centerline $(x, y=0, z=0)$ from the time a plume segment is released until the vertical distribution of the segment becomes uniform between the ground and the capping inversion layer (becomes well mixed in the vertical direction):

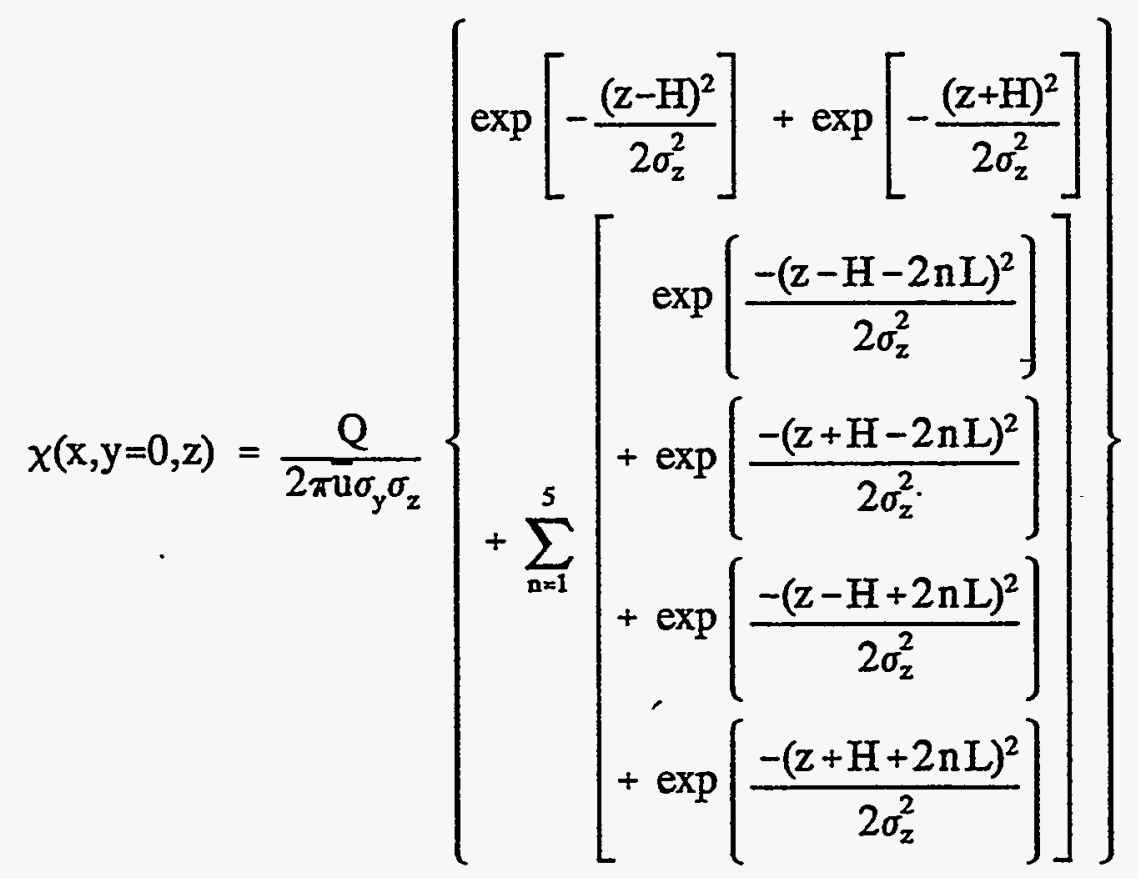

where

$$
\begin{aligned}
& \chi(\mathrm{x}, 0, \mathrm{z})=\text { time-integrated air concentration }\left(\mathrm{mg}-\mathrm{s} / \mathrm{m}^{3}\right) \text { at the } \\
& H=h+\Delta h \text {, the height of the plume centerline }(m) \text {; } \\
& \mathrm{L}=\text { height (m) of the capping inversion layer (mixing height). }
\end{aligned}
$$


In CHEM_MACCS, only five terms of the summation in Equation (2.11) are considered since subsequent terms are expected to be small. ${ }^{18}$

In the ATMOS module of CHEM_MACCS, off-centerline concentrations are not calculated. This is done in the EARLY module of CHEM_MACCS (see Sections 3.1.1 and 3.2.1). The off-centerline concentrations required for dose calculations are calculated using the plume centerline air concentration or the ground-level air concentration under the plume centerline and the appropriate off-centerline correction factors.

At each spatial interval along the plume's trajectory, CHEM_MACCS tests for the occurrence of a uniform distribution in the vertical direction (a well-mixed plume between the ground and the capping inversion layer). Once a uniform vertical distribution is attained, the following simple Gaussian equation is used to calculate centerline air concentrations ${ }^{18}$ :

$$
\chi(x, y=0, z)=\frac{Q}{\sqrt{2 \pi} \overline{\mathrm{u}} \sigma_{\mathrm{y}} \mathrm{L}}
$$

where $\mathrm{x}, \mathrm{y}, \mathrm{z}, \mathrm{Q}, \mathrm{u}$, and $\sigma_{\mathrm{y}}$ have definitions unchanged from those given for Equation (2.11) and $\mathrm{L}$ is the mixing height (m).

A heuristic test is used to determine when the plume becomes well mixed between the ground and the inversion lid. Two conditions must be met to pass the test: (1) $\sigma_{z}$ must be larger than $\mathrm{H}$, the height of the plume centerline, and (2) the ground-level centerline air concentration calculated using the uniform mixing Equation (2.12) must exceed the ground-level centerline air concentration calculated using the multiple reflection equation, Equation (2.11). Once uniform mixing in the vertical direction is attained, the multiple reflection equation is no longer used. Thereafter, there is no need to calculate $\sigma_{z}$.

\subsubsection{Dispersion Parameters}

The rate at which materials disperse in the atmosphere depends strongly on atmospheric turbulence, which varies greatly with stability class. Therefore, the rate of expansion of a plume during downwind transport will also vary with stability class.

The growth of plume dimensions during downwind transport over short distances $(1 \mathrm{~km})$ has been experimentally determined over flat terrain covered by prairie grass (surface roughness height $z_{0}=3 \mathrm{~cm}$ ) for short plumes (10-min release times) released during stable, neutral, and unstable atmospheric conditions. ${ }^{28}$ Pasquill used these data to develop curves that depict the increase of plume dimensions ( $\sigma_{\mathrm{y}}$ and $\sigma_{\mathrm{z}}$ values) with downwind distance for each of the six Pasquill-Gifford (P-G) stability classes A through $\mathrm{F}^{29}$ Although measurements were only made to $1 \mathrm{~km}$, Pasquill extrapolated the curves to $100 \mathrm{~km}$. These curves, as later modified by Gifford, ${ }^{30,31}$ are presented in Figure 2. 

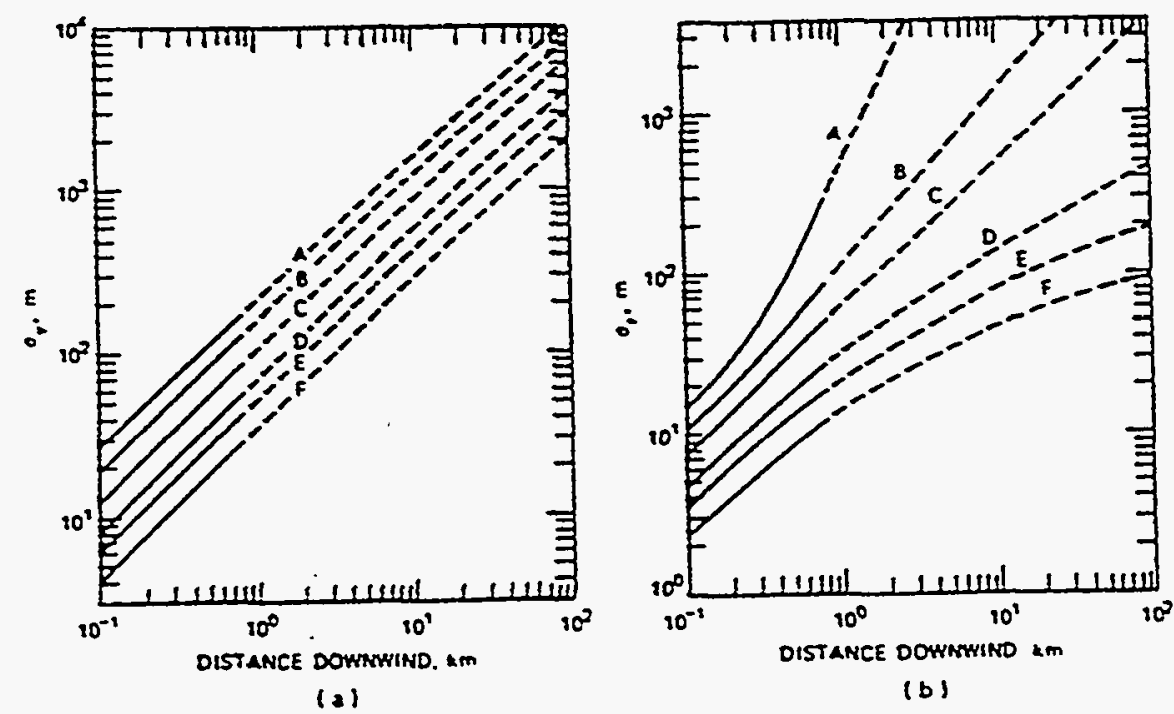

Figure 2 Dependence of $\sigma_{y}$ and $\sigma_{z}$ on distance for the six Pasquill-Gifford stability classes A through $F$ (solid lines depict the range of the experimental data)

Tadmor and Gur have constructed analytical fits to the Pasquill-Gifford curves depicted in Figure 2.32 The fits have the following functional form:

$$
\sigma_{y i}=a_{i} x^{b_{i}} \text { and } \sigma_{z i}=c_{i} x^{d_{i}}
$$

where $\mathrm{i}$ denotes the prevailing stability class (Pasquill-Gifford classes A through F correspond to $i$ values 1 through 6 ) and the values in Table 2 for the constants $a_{i}$ through $\mathrm{d}_{\mathrm{i}}$ are supplied on the sample ATMOS input file. The values of the constants in the table correct the typographical errors identified by Dobbins. ${ }^{33}$

Table 2 Values of constants for $\sigma_{\mathrm{y}}$ and $\sigma_{\mathrm{z}}$ equations

\begin{tabular}{c|c|c|c|c|c}
\hline \multicolumn{2}{c|}{ Stability Class } & \multicolumn{4}{|c}{ Constant } \\
\hline P-G & $\mathrm{i}$ & $\mathrm{a}_{\mathrm{i}}$ & $\mathrm{b}_{\mathrm{i}}$ & $\mathrm{c}_{\mathbf{i}}$ & $\mathrm{d}_{\mathrm{i}}$ \\
\hline $\mathrm{A}$ & 1 & 0.3658 & 0.9031 & 0.00025 & 2.125 \\
$\mathrm{~B}$ & 2 & 0.2751 & 0.9031 & 0.0019 & 1.6021 \\
$\mathrm{C}$ & 3 & 0.2089 & 0.9031 & 0.2 & 0.8543 \\
$\mathrm{D}$ & 4 & 0.1474 & 0.9031 & 0.3 & 0.6532 \\
$\mathrm{E}$ & 5 & 0.1046 & 0.9031 & 0.4 & 0.6021 \\
$\mathrm{~F}$ & 6 & 0.0722 & 0.9031 & 0.2 & 0.6020 \\
\hline
\end{tabular}


As used in CHEM_MACCS, the values of the dispersion parameters, $\sigma_{y}$ and $\sigma_{z}$ in Equations (2.10), (2.11), and (2.12) must change continuously although not necessarily smoothly. Since stability class changes discretely, whenever stability class changes, the source distance $x$ in the dispersion parameter equation [Equation (2.13)] must be changed to some new value that causes dispersion parameter growth to be continuous. The new value of the source distance is called the "virtual source" distance. It has a different value for $\sigma_{y}$ and for $\sigma_{z}$. It is calculated as follows. Let $i$ be the stability class before the change in atmospheric conditions, $j$ the stability class after the change, $x_{y i}$ and $x_{z i}$, the source distances under the old conditions (downwind distances of the plume when the stability class changes; if this is the first change in stability class, then $x_{y i}=x_{z i}$ ), and $x_{y j}$ and $x_{2 j}$ the source distances under the new conditions (i.e., the virtual source distances).

To. ensure continuity, $\sigma_{\mathrm{yi}}$ must equal $\sigma_{y \mathrm{j}}$, and $\sigma_{\mathrm{zi}}$ must equal $\sigma_{\mathrm{zj}}$. Thus,

$$
\begin{aligned}
& a_{i}\left(x_{y i}\right)^{b_{i}}=\sigma_{y i}=\sigma_{y j}=a_{j}\left(x_{y j}\right)^{b_{j}} \\
& c_{i j}\left(x_{z i}\right)^{d_{i}}=\sigma_{z i}=\sigma_{z j}=c_{j}\left(x_{z i}\right)^{d}
\end{aligned}
$$

which after solving for the new "virtual source" distances gives

$$
\begin{aligned}
& x_{y j}=\left[\frac{1}{a_{j}} a_{i}\left(x_{y i}\right)^{b_{i}}\right]^{1 / b_{j}}=\left[\frac{\sigma_{y i}}{a_{j}}\right]^{1 / b_{j}} \\
& x_{z j}=\left[\frac{1}{c_{j}} c_{j}\left(x_{z i}\right)^{d_{j}}\right]^{1 / d_{j}}=\left[\frac{\sigma_{z i}}{c_{j}}\right]^{1 / d_{j}}
\end{aligned}
$$

Figure 3 illustrates the growth of $\sigma_{y}$ over three time periods characterized by stability classes $\mathrm{i}, \mathrm{j}$, and $\mathrm{k}$, during which $\sigma_{\mathrm{y}}$ first grows at a moderate rate, then slowly, and finally rapidly.

Finally, although new "virtual source" distances for $\sigma_{y}$ and $\sigma_{z}$ are calculated every time stability class changes, these distances are used only to calculate growth of $\sigma_{y}$ and $\sigma_{z^{*}}$. Plume locations are always expressed relative to the release point that is the center point of the polar coordinate computational grid.

For a given spatial element, the average values of $\sigma_{\mathrm{y}}$ and $\sigma_{\mathrm{z}}$ are used in calculating air and ground concentrations for the entire spatial element. The average values of $\sigma_{\mathrm{y}}$ and $\sigma_{z}$ are the arithmetic means of the initial and final values of these two parameters as a plume segment traverses the spatial element. 


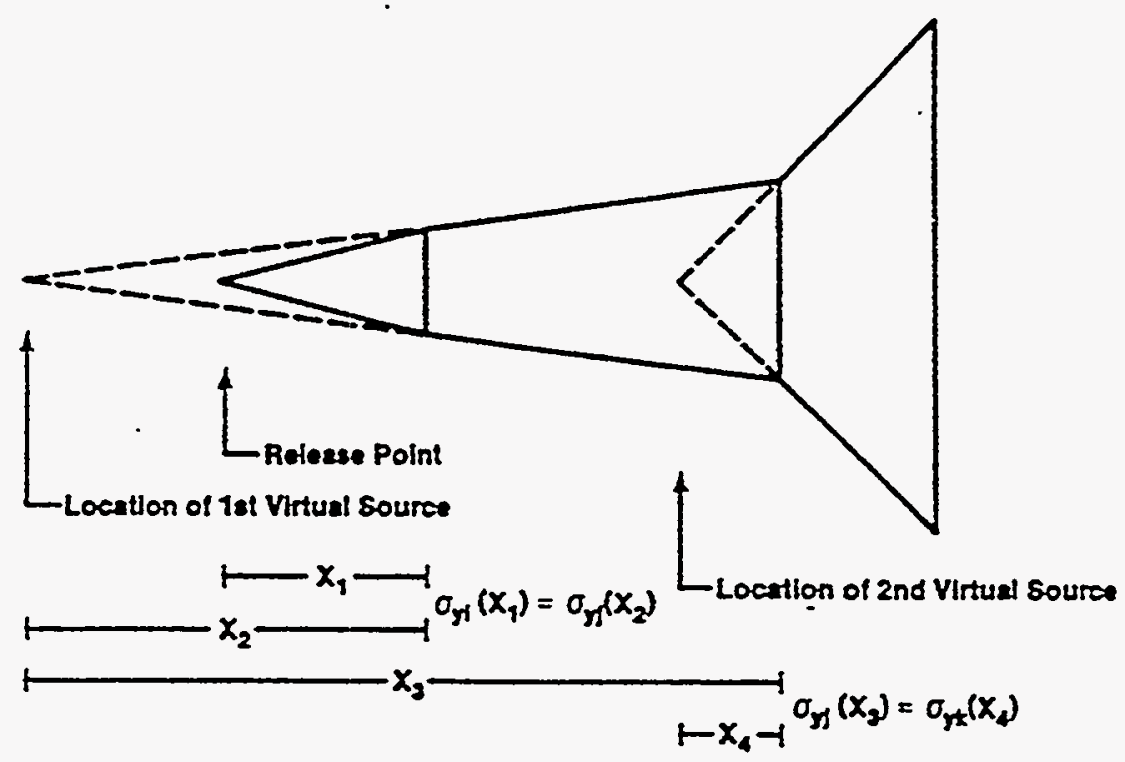

Figure 3 Growth of $\sigma_{y}$ during three time periods characterized by different atmospheric stabilities $i, j$, and $k$

\subsubsection{Surface Roughness}

The Pasquill-Gifford curves depicted in Figure 2 are appropriate for transport over flat terrain covered by prairie grass (surface roughness height $z_{0}=3 \mathrm{~cm}$ ). However, plume transport will usually be over areas characterized by surface roughness heights greater than $3 \mathrm{~cm}$. Table 3 presents some approximate heights for different surfaces. $27,34,35$

Table 3 Approximate roughness heights $\left(\mathrm{z}_{0}\right)$ for different surfaces

\begin{tabular}{l|c}
\hline Type of Surface & $z_{0}(\mathrm{~cm})$ \\
\hline Lawns & 1 \\
Tall grass, crops & $10-15$ \\
Countryside & 30 \\
Suburbs & 100 \\
Forests & $20-200$ \\
Cities & $100-300$ \\
\hline
\end{tabular}


Surface roughness principally affects vertical dispersion and thus $\sigma_{z}$ values. The following formula can be used to correct Pasquill-Gifford values of $\sigma_{2, \mathrm{P}-G}$, which are appropriate for $z_{0}=3 \mathrm{~cm}$, for the effects of rougher surfaces ${ }^{36}$ :

$$
\sigma_{z, \text { eCW }}=\sigma_{z, P-G}\left[\frac{z_{0, \text { Dew }}}{z_{0, P-G}}\right]^{0.2}
$$

Table 3 suggests that a roughness length greater than $3 \mathrm{~cm}$, at least $10 \mathrm{~cm}$ and possibly $100 \mathrm{~cm}$, is more likely typical of populated areas. If $z_{0, \text { new }}=10 \mathrm{~cm}$ and $z_{0, P-G}=3 \mathrm{~cm}$, then $\sigma_{\mathrm{z} \text {, new }}=1.27 \sigma_{\mathrm{z}, \mathrm{P}-\mathrm{G}}$. Since in CHEM_MACCS both $\sigma_{\mathrm{y}}$ and $\sigma_{\mathrm{z}}$ can be scaled by changing the value of an input scale factor, surface roughness effects on $\sigma_{z}$ can be corrected by changing the value of the $\sigma_{z}$ scale factor. In fact, in the sample ATMOS input file, the value of the $\sigma_{\mathrm{z}}$ scale factor is set to 1.27 to scale Pasquill-Gifford values of $\sigma_{z}$ up to values appropriate for surfaces characterized by 10 -cm roughness lengths.

\subsubsection{Plume Meander}

Both theoretical and experimental studies indicate that as sampling (measurement) times $(\tau)$ increase, maximum values of plume concentrations $\left(\chi_{\max }\right)$ for continuous (stack) plumes measured at some fixed downwind location decrease, ${ }^{37,38,39}$ because meander in the horizontal and vertical directions increases the effective lateral and vertical dimensions of the plume. Specifically, $\chi_{\max }$ is proportional to $\tau^{-p}$ where $p=0.5$ for sampling times greater than $1 \mathrm{hr}$. Since $\chi_{\max }$ is proportional to $\left(\sigma_{\mathrm{y}} \sigma_{\mathrm{z}}\right)^{-1}$, if $\sigma_{\mathrm{y}}$ and $\sigma_{\mathrm{z}}$ are of similar magnitude, then $\sigma_{\mathrm{y}} \approx \sigma_{\mathrm{z}} \propto \tau^{\mathrm{m}}$ where $\mathrm{m}=0.25$, which has been confirmed experimentally for $\sigma_{\mathrm{y} .}{ }^{37}$ Now, since for puff releases, release duration may be equated to sampling time, the increase in effective plume dimensions (values of $\sigma_{y}$ and $\sigma_{z}$ ) with release time (effects of plume meander) can be calculated using the following equation,

$$
\sigma_{y}=\sigma_{\text {ref }}\left[\frac{\Delta t}{\Delta t_{r e f}}\right]^{m}
$$

where $\sigma_{\text {ref }}$ and $t_{\text {ref }}$ are the sigma value and release duration (10 $\mathrm{min}$ for the experiments that support the Pasquill-Gifford curves) of the reference plume, $t$ is the release time of the long duration release, and as recommended by Gifford, $\mathrm{m}$ equals 0.2 for release durations less than $1 \mathrm{hr}$ and 0.25 for release durations greater than $1 \mathrm{hr} .{ }^{30}$

In CHEM_MACCS, Equation (2.17) is used to model the increase in $\sigma_{y}$ caused by lateral meandering of the plume, which increases with increasing release duration. Vertical meander is neglected since it is expected to become unimportant shortly after release is completed (within $30 \mathrm{~min}$ ). ${ }^{37}$ As implemented in CHEM_MACCS, the user must specify values of $\mathrm{m}$ for short and long releases and the release durations to which they apply. The values recommended by Gifford are specified in the sample input file for ATMOS provided with CHEM_MACES. 


\subsection{Plume Depletion}

Deposition onto the ground is caused by washout and by gravitational settling onto, impaction on, and diffusion to surfaces. The deposition caused by rain is called wet deposition. Deposition not caused by rain is called dry deposition. In CHEM_MACCS, all of these processes are modeled as first-order rate processes.

\subsubsection{Dry Deposition}

Dry deposition is modeled using Chamberlain's source depletion method modified to allow treatment of a particle size distribution and capping of vertical expansion by an inversion lid. ${ }^{40,41,42}$ The source depletion method calculates the rate at which materials are deposited onto the ground (the deposition flux) as the product of the ground-level air concentration of the materials and the dry deposition velocity of those materials. ${ }^{20}$ The method neglects the effects of deposition on the vertical distribution of the plume. Thus, dry deposition does not perturb the normal distribution of plume materials in the vertical direction. This is a reasonable assumption when vertical mixing is efficient (i.e., when neutral or unstable atmospheric conditions prevail). When stable conditions occur, the assumption introduces an artificial flux of material from upper regions of the plume to regions near the ground.

The ground-level air concentration at a location $(x, y, z=0)$ of a plume capped by an inversion lid at height $L$ is obtained from Equation (2.11) by multiplying it by the normal distribution function along the $y$-axis, $\exp \left(-y^{2} / 2 \sigma_{y}^{2}\right)$, and setting $z=0$, which yields

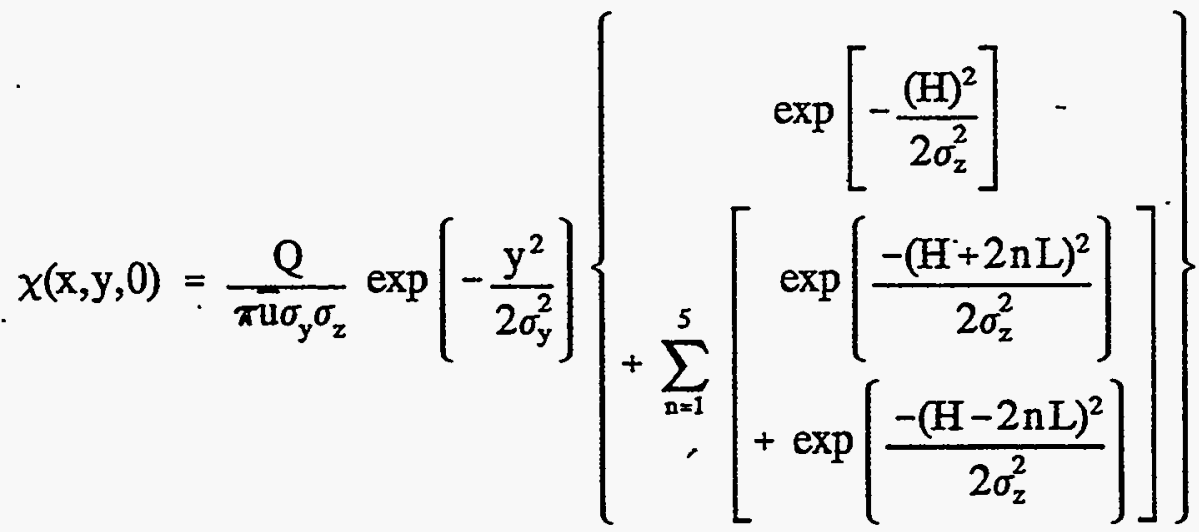

$$
\begin{aligned}
& =\frac{\mathrm{Q}}{\pi \sigma_{y} \sigma_{z} \bar{u}} \exp \left[-\frac{y^{2}}{2 \sigma_{y}^{2}}\right][F]
\end{aligned}
$$

where $F$ is the sum of all of the exponential terms that contain $\sigma_{z}$ (the terms in the larger set of brackets).

The flux of plume material to the ground, $\omega(x, y)$, is given by

$$
\omega(\mathrm{x}, \mathrm{y})=\mathrm{v}_{\mathrm{d}} \chi(\mathrm{x}, \mathrm{y}, 0)
$$


where $v_{d}$, the dry-deposition velocity, embodies the combined effects of gravitational settling of materials onto, impaction on, and diffusion to the ground. ${ }^{20}$

The rate of loss of plume materials $(\mathrm{dQ} / \mathrm{dx})$ by dry deposition to the ground into a differential length $d x$ located at the downwind distance $x$ is given by ${ }^{41}$ :

$$
\frac{\mathrm{dQ}}{\mathrm{dx}}=-\int_{-\infty}^{\infty} \omega(\mathrm{x}, \mathrm{y}) \mathrm{dy}
$$

Substitution of Equations (2.18) and (2.19) into Equation (2.20) now gives

$$
\frac{d Q}{d x}=-F \sqrt{\frac{2}{\pi}} \frac{v_{d} Q}{\sigma_{z} \bar{u}} \int_{-\infty}^{\infty} \frac{1}{\sqrt{2 \pi} \sigma_{y}}\left[\exp \left[-\frac{y^{2}}{2 \sigma_{y}^{2}}\right]\right] d y=-F\left[\frac{2}{\pi}\right]^{1 / 2} \frac{v_{d} Q}{\sigma_{z}^{\bar{u}}}
$$

since the value of the integral is one. During any single hour of weather data, the mean windspeed, $u$, is constant. Thus $d x=u d t$, and using this into Equation (2.21) gives

$$
\frac{\mathrm{dQ}}{\mathrm{dt}}=-\mathrm{F}\left[\frac{2}{\pi}\right]^{1 / 2} \frac{\mathrm{v}_{\mathrm{d}} \mathrm{Q}}{\sigma_{\mathrm{z}}}
$$

Rearranging and integrating gives

$$
\frac{\mathrm{Q}}{\mathrm{Q}_{0}}=\mathrm{f}_{\mathrm{d}}=\exp \left[-\frac{\mathrm{v}_{\mathrm{d}} \Delta t}{\mathrm{~F}^{\prime}}\right]
$$

where

$$
\mathrm{F}^{\prime}=\left[\frac{2}{\pi}\right]^{1 / 2} \sigma_{\mathrm{z}} \frac{1}{\mathrm{~F}}
$$

and $f_{d}$ is the fraction of material in the plume at the beginning of the time period $(\Delta t)$ that is not removed by dry deposition during the time period. Since $d Q / d t=-v_{d}(A / V) Q$, where $\mathrm{A}$ is the deposition surface area (area onto which dry deposition is occurring) and $\mathrm{V}$ is the plume volume (volume from which deposition is occurring), $\mathrm{A} / \mathrm{V} .=1 / \mathrm{z}$ where $z=F^{\prime}$ is the height of the column from which deposition is occurring, here an effective plume height. As noted in the Reactor Safety Study, ${ }^{21}$

$$
\bar{z}=\frac{\int^{\infty} \chi(x, 0, z) d z}{\chi(x, 0,0)}=\text { effective height of plume }
$$

Finally, once the plume has become well mixed below the inversion lid, $\mathrm{V}=\mathrm{AL}$, so $\mathrm{dQ} / \mathrm{dt}=-\mathrm{v}_{\mathrm{d}}(\mathrm{A} / \mathrm{AL}) \mathrm{Q}=-\mathrm{v}_{\mathrm{d}} \mathrm{Q} / \mathrm{L}$, and thus $\mathrm{z}=\mathrm{L}$. 
In CHEM_MACCS, the effect of particle size on dry deposition velocity is treated by dividing the particle size range of the materials subject to dry deposition into $i$ sections, specifying the fraction $\left(f_{i}\right)$ of all aerosol materials in each section, assigning a dry deposition velocity $\left(v_{\mathrm{di}}\right)$ to each size section, and applying Equation (2.23) separately to each section. Thus,

$$
f_{d i}=\frac{Q_{i}}{Q_{o i}}=\exp \left[-\frac{v_{d i} \Delta t}{\bar{z}}\right]
$$

where $Q_{o i}$ is the amount of aerosol in section $i$ transported into the spatial element, $Q_{i}$ is the amount transported out of the element, $\mathrm{f}_{\mathrm{di}}=\mathrm{Q}_{\mathrm{i}} / \mathrm{Q}_{\mathrm{oi}}$ is the fraction remaining after the plume segment traverses the spatial element, $\Delta t$ is the time required for the segment to traverse the spatial element, and $\mathrm{z}$ is the effective height of the segment.

Finally, since $Q=\Sigma_{i} Q_{i}, Q_{i}=Q_{o i} f_{d i}$, and $Q_{o i}=Q_{0} f_{i}$, the fraction $\left(f_{d}\right)$ of all aerosol materials in all of the size sections $i$ that remains after dry deposition has occurred from each section onto the entire spatial interval is given by

$$
f_{d}=\frac{Q}{Q_{0}}=\frac{1}{Q_{0}} \sum_{i} Q_{i}=\frac{1}{Q_{0}} \sum_{i} Q_{0 i} f_{d i}=\frac{1}{Q_{0}} \sum_{i} Q_{0} f_{i} f_{d i}=\sum_{i} f_{i} f_{d i}
$$

When dry deposition is calculated in CHEM_MACCS, $\Delta t$ in Equation (2.25) is taken to be the time required for the representative weather point of the plume segment to cross the spatial element. Thus, $\Delta t$ equals the time of arrival of the segment's representative weather point at the far side of the spatial element minus the time of its arrival at the near side of the element. See Section 2.11 for the-treatment of dry deposition (Equation 2.26) in calculating the air and ground concentrations.

Because CHEM_MACCS allows plume segments to have release durations as long as $10 \mathrm{hr}$, lengthy plume segments, which during transport lie over more than one spatial element, are common. Of course, when exiting a spatial element, even a short plume segment will lie over at least two spatial elements. Even when a plume segment lies above more than one spatial element, dry deposition from the segment is assumed to occur entirely onto the spatial element that the segment's representative weather point is above. Thus, dry deposition always occurs only onto the element that the representative weather point of the segment is traversing, no matter how many elements lie under the entire length of the segment.

After a segment has traversed a spatial element, the amounts of material in each size section are decreased by the amounts removed from the section by dry deposition during transport across the spatial element. Then, the fractions that specify the amounts of aerosol materials in the sections of the aerosol size distribution are recalculated and, as a consistency check, their sum is renormalized to one. 


\subsubsection{Wet Deposition} Vogt ${ }^{19}$ :

Wet deposition, that is, washout, is calculated using the model of Brenk and

$$
\frac{\mathrm{dQ}}{\mathrm{dt}}=-\Lambda \mathrm{Q}=-\mathrm{aI}{ }^{\mathrm{b}} \mathrm{Q}
$$

where

$$
\begin{array}{ll}
\Lambda= & \text { the washout coefficient }\left(\mathrm{s}^{-1}\right), \\
\mathrm{I}= & \text { precipitation intensity }(\mathrm{mm} / \mathrm{hr}), \text { and } \\
\mathrm{a}, \mathrm{b}= & \text { dimensionless constants (in the ATMOS sample input file } \\
\left.\mathrm{a}=9.5 \times 10^{-5} \text { and } \mathrm{b}=0.8\right) .{ }^{43}
\end{array}
$$

Rearrangement and integration of Equation (2.27) gives

$$
\frac{\mathrm{Q}}{\mathrm{Q}_{0}}=\mathrm{f}_{\mathrm{w}}=\exp \left(-\mathrm{aI}^{\mathrm{b}} \Delta \mathrm{t}\right)
$$

where

$\mathrm{Q}_{0} \quad=\quad$ amount of chemical species (mg) transported into the spatial element,

$\mathrm{Q}=$ amount of chemical species (mg) transported out of the element,

$\Delta \dot{\mathrm{t}}=\mathrm{time}(\mathrm{s})$ that the plume segment takes to cross the spatial element, and

$\mathrm{f}_{\mathrm{w}}=\mathrm{Q} / \mathrm{Q}_{0}$, the fraction of aerosol material in the segment at the beginning of the time period $\Delta t$ that is not removed by wet deposition during the time period.

Unlike dry deposition, which is a continuous and relatively slow process, wet deposition is not continuous and often is quite rapid, at least compared with dry deposition. Therefore, washout of all of the materials in a plume segment onto the spatial element that lies under the segment's representative weather point is not acceptable. Instead, washout should be apportioned over all of the spatial elements that lie under the segment.

For example, consider the release of a plume segment during a 10-hr period during which the average windspeed is $5 \mathrm{~m} / \mathrm{s}$. At the end of the release, the plume segment will have a length of $180 \mathrm{~km}$ and during the early stages of a CHEM_MACCS calculation will extend over many spatial intervals. Suppose the windspeed now drops to $1 \mathrm{~m} / \mathrm{s}$ and that a 1 -hr-long rainstorm begins just as the segment's representative weather point enters a $3.6-\mathrm{km}$-long spatial element. The storm will end just as the representative weather point leaves the spatial element. If wet deposition occurs only onto the spatial element under the representative weather point, all materials washed out 
from the segment will be deposited onto only one spatial element even though the segment most likely covers several spatial elements on the standard CHEM_MACCS computational grid. This would produce very high ground concentrations in the single spatial element onto which deposition would occur, concentrations possibly appropriate for areas lying under rain cells, but most likely not representative of the average ground concentrations over all areas (spatial elements) beneath the segment during the storm.

To apportion wet deposition over all of the spatial elements traversed wholly or partly by a plume segment during a rainstorm, on an hourly basis, the average value of the fraction of the segment's length that lies over each element that the segment traverses during the course of the storm $\left(f_{x x}\right)$ miust be calculated. Let $L_{S I}$ be the length of the portion of the plume segment that lies over one of the spatial intervals beneath the plume at time $t$. Figure 4 is a plot of $L_{S I}$ versus $t$ for a segment of total length $L_{s}$ and an interval of radial length $L_{1}$ (length in the downwind direction), where $I_{s} \neq L_{1}$. Because $\mathrm{L}_{\mathrm{s}} \neq \mathrm{L}_{\mathrm{I}}$, the plot has a trapezoidal shape with height $\mathrm{I}_{\operatorname{mex}}=\min \left(\mathrm{L}_{\mathrm{s}}, \mathrm{L}_{\mathrm{J}}\right)$. Note that when $\mathrm{L}_{s}=\mathrm{L}_{\mathrm{I}}$, the trapezoid is reduced to a triangle.

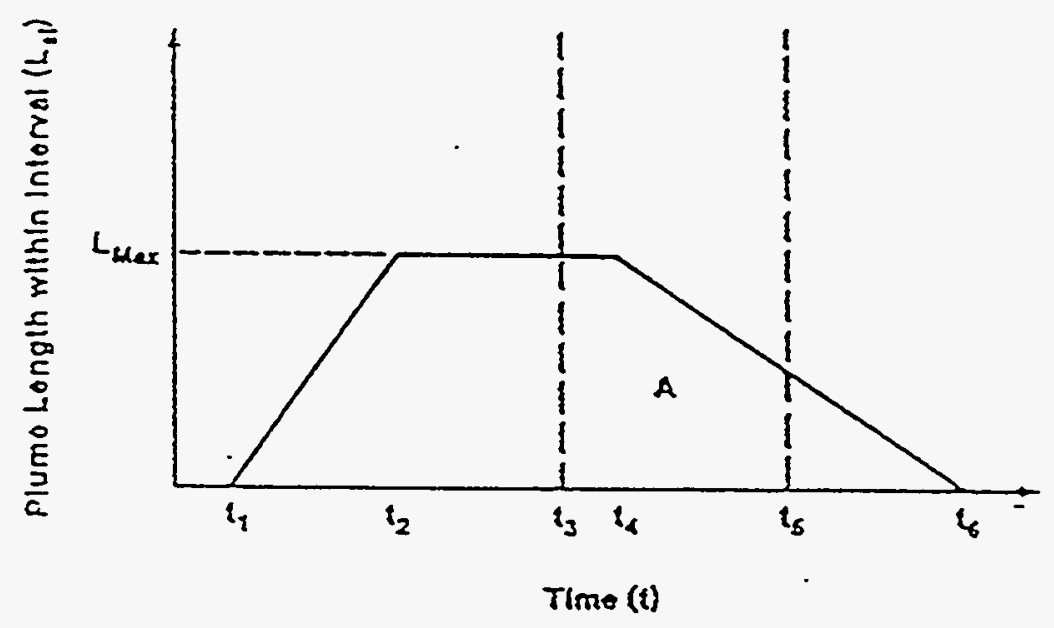

Figure 4 Temporal dependence of the portion of a plume's length that is over a spatial element.

In Figure $4, t_{1}$ is the time when the head of the plume segment enters the spatial element; $t_{6}$ is the time when the tail of the segment leaves the element. If $L_{S}<L_{1}, t_{2}$ is the time when the tail of the segment enters the element and $t_{4}$ the time when the head leaves the element; if $L_{S}>L_{1}, t_{2}$ is the time when the head of the segment leaves the element and $t_{4}$ is the time when the tail enters the element. The time points $t_{3}$ and $t_{5}$ indicated by vertical dashed lines denote the beginning and end of a 1-hr time period $\left(t_{5}-t_{3}=1 \mathrm{hr}\right)$ during which weather data are constant.

Since windspeed has only one value during any hour of weather data, the geometric shape bounded by the dashed lines will always be regular, no matter where the hourly time points fall. Let the area of this regular shape be $A$. Then, since $L_{S}$ and $L_{1}$ and thus $L_{\max }$ are known, and all of the time points on the figure (points $t_{1}$ through $t_{6}$ ) are known 
or are calculated by CHEM_MACCS, A can be calculated. Thus, for any 1-hr time period, $L_{a v}$, the average value of $L_{s I}$ during that time period is given by $L_{\alpha v}=A /\left(t_{5}-t_{3}\right)$ $=A$, since $t_{5}-t_{3}=1 \mathrm{hr}$; and the hourly average value of the fraction of the segment's length that lies over each element that the segment traverses during the course of the rainstorm $\left(f_{x v}\right)$ is given by $f_{x v}=L_{x v} L_{s}$. So $f_{2 v}$ can be calculated hour by hour for each plume segment and every spatial element.

The quantity $f_{\mathrm{av}}$ now must be introduced into Equation (2.28). To see how this is done, let $\Delta t$ be the time required to transport a plume segment across a given spatial element $\mathrm{k}$. If that time is several hours long, then

$$
\Delta t=\sum_{j} \Delta t_{j}
$$

where the first and last values of $\Delta t_{j}$ can be fractional hours. Let $\left\{f_{2 v, j}\right\}$ be the set of hourly values of $f_{x v, j}$ that cover the time period $\Delta t$ during which the plume segment is transported across the spatial element; let $Q_{j}$ and $Q_{j+1}$ be the amounts of aerosol material in the entire plume (not just the portion over the spatial element) at the beginning and the end of time step $j$; and let $I_{j}$ be the rain intensity during time step $j$. Because weather data are constant during any hour, $\mathrm{I}_{\mathrm{j}}$ is single valued during any time step $\mathrm{j}$. But during time step $j$, the rate of wet deposition from the entire plume is given by $\Lambda_{j}=a\left(I_{j}\right)^{b}$. Therefore, the rate of wet deposition onto the spatial element is given by $\Lambda_{\mathrm{j}} f_{\mathrm{avj}}$. Thus $f_{\text {wj }}$, the fraction of aerosol material remaining in the plume after deposition during a time step, is given by

$$
f_{w j}=\exp \left(-\Lambda_{j} f_{a v, j} \Delta t_{j}\right)=\exp \left[-a\left(I_{j}\right)^{b} f_{w, j} \Delta t_{j}\right]
$$

$f_{w}$, the fraction of aerosol remaining in the plume after deposition only onto the spatial element during all of the time steps $j$ required to transport the plume segment across the spatial element, is given by

$$
f_{w}=\prod_{j} f_{w j}
$$

\subsection{Centerline Air and Ground Concentrations}

As modeled in CHEM_MACCS, dry and wet deposition are independent processes. For example, assume that during transport across a spatial element dry deposition alone would deplete a plume segment of one-tenth of the material in the segment, and wet deposition alone would remove one-half of the material in the segment. Then, if the two processes are independent and occur simultaneously, the fraction of the material in the segment upon entry into the element, which remains when the segment leaves the element, is $0.45=(1.0-0.1)(1.0-0.5)$. Thus, the total amount of material $\left(\Delta \mathrm{Q}_{1}\right)$ deposited onto the ground during transport of a plume segment across a spatial element $\mathrm{k}$ is given by 


$$
\Delta Q_{k}=Q_{x}\left(1-f_{d} f_{w}\right)
$$

where $\mathrm{Q}_{\mathrm{r}}$ is the amount of airborne aerosol material that is transported into interval $\mathrm{k}$ by the plume segment, $f_{d}$ and $f_{w}$ are the fractions of material that would remain in the plume after transport across the spatial element if only dry deposition or only wet deposition occurred, and $f_{d}$ and $f_{w}$ are calculated using Equations (2.26) and (2.30). Now let $\mathrm{GC}_{\mathrm{k}}(\mathrm{y}=0)$ be the average ground concentration under the plume centerline along the length $\left(L_{k}\right)$ of spatial interval $k$. Then

$$
\begin{aligned}
\Delta \mathrm{Q}_{\mathrm{k}} & =\mathrm{L}_{\mathrm{k}} \mathrm{GC}_{\mathrm{k}}(\mathrm{y}=0) \int_{-\infty}^{\infty} \exp \left[-\frac{\mathrm{y}^{2}}{2 \sigma_{y}^{2}}\right] d y \\
& =\sqrt{2 \pi} \sigma_{\mathrm{y}} \mathrm{L}_{\mathrm{k}-\mathrm{G}} \mathrm{GC}_{\mathrm{k}}(\mathrm{y}=0) \int_{-\infty}^{\infty} \frac{1}{\sqrt{2 \pi} \sigma_{\mathrm{y}}} \exp \left[-\frac{\mathrm{y}^{2}}{2 \sigma_{y}^{2}}\right] d y \\
& =\sqrt{2 \pi} \sigma_{\mathrm{y}} \mathrm{L}_{\mathrm{k}} \mathrm{GC}_{\mathrm{k}}(\mathrm{y}=0)
\end{aligned}
$$

since the value of the second integral is one. Accordingly,

$$
\mathrm{GC}_{\mathrm{r}}(\mathrm{y}=0)=\frac{\Delta \mathrm{Q}_{\mathrm{k}}}{\sqrt{2 \pi} \sigma_{\mathrm{y}} \mathrm{L}_{\mathrm{k}}}
$$

Average values for $\sigma_{y}, \sigma_{z}$, plume height $\mathrm{H}$, and average windspeed $\mathrm{u}$ for transport of a plume segment across spatial interval $\mathrm{k}$ are given by

$$
\begin{aligned}
\sigma_{y, a v} & =0.5\left(\sigma_{y, k}+\sigma_{y, k+1}\right) \\
\sigma_{z, a v} & =0.5\left(\sigma_{z, k}+\sigma_{z, k+1}\right) \\
H & =0.5\left(H_{k}+H_{k+1}\right) \\
\bar{u}_{z v} & =\frac{\mathrm{L}_{k}}{t_{k+1}-t_{k}}
\end{aligned}
$$

where the subscripts $k$ and $k+1$ signify that the value of the parameter pertains respectively to the near and far sides of the spatial interval (the values when the segment enters and leaves the element), and $L_{k}$ is the length of the element. Accordingly, the average plume centerline air concentration, $\mathrm{AC}_{\mathrm{x}}(\mathrm{y}=0, \mathrm{z}=\mathrm{H})$, of the plume segment during transport across spatial element $\mathrm{k}$ is

$$
\mathrm{AC}_{\mathrm{z}}(\mathrm{y}=0, \mathrm{z}=\mathrm{H})=\left[\frac{\chi}{\mathrm{Q}}\right]_{\mathrm{av}}\left[\mathrm{Q}_{\mathrm{k}}-0.5 \Delta \mathrm{Q}_{\mathrm{k}}\right]
$$


Here $[\chi / Q]_{\mathrm{zv}}$ is calculated at the plume centerline using average values for $\sigma_{\mathrm{y}}, \sigma_{\mathrm{z}}$, and $\overline{\mathrm{u}}$ is defined by Equation (2.34) substituted into either the multiple reflection Equation (2.11) or vertically well-mixed Equation (2.12) Gaussian plume equations defined previously. Finally, the amount of material still airborne in the plume segment after transport across the spatial element is $\mathrm{Q}_{x+1}=\mathrm{Q}_{x}-\Delta \mathrm{Q}_{\mathrm{r}}$.

\subsection{Results Calculated by ATMOS}

Consider a CHEM_MACCS calculation that examines a release composed of only one plume segment. For each weather trial examined during the calculation, ATMOS calculates values at the midpoint of each spatial element traversed by the segment for the following quantities: the arrival time of the leading edge (head) of the segment; the duration overhead of the segment (the arrival time at the spatial element's midpoint of the segment's tail minus that of the segment's head); average values of $\sigma_{y}, \sigma_{z}, \mathrm{H}$, and $\overline{\mathrm{u}}$ calculated using Equation (2.34); the ratio of the air concentration at ground level under the centerline to the centerline air concentration calculated using the average values of $\sigma_{y}, \sigma_{z}, \mathrm{H}$, and $\overline{\mathrm{u}}$; and the angular width of the segment. In addition, for each chemical specified for the calculation, ATMOS uses Equations (2.35) and (2.33) to calculate for each spatial element the average plume centerline air concentration and the average ground concentration under the centerline. If the release used in the calculation is composed of several plume segments, then all of these quantities are calculated for each segment of the release. 
This page intentionally left blank. 


\section{Exposure Pathways}

\subsection{Off-Centerline Correction Factors}

The ATMOS module calculates the air concentration at the plume centerline, the ground concentration under the plume centerline, and the ratio of ground-level air concentration under the plume centerline to the air concentration at the plume centerline. This ratio is multiplied by the plume centerline air concentration to obtain the ground-level air concentration under the plume centerline. These three concentrations, namely, the plume centerline air concentration, the ground-level air concentration under the plume centerline, and the ground concentration under the plume centerline, are used in the dose equations discussed in this chapter.

To calculate the chemical doses at the off-centerline region of various spatial elements, these centerline concentrations are modified by the appropriate off-centerline correction factors depending on where the spatial elements are located. A spatial element is specified by its radial interval number and its compass direction sector.number. The spatial element is also called the coarse spatial element.

For people in the process of evacuation, the "top-hat" function shown in Figure 5 is used to approximate the Gaussian crosswind distribution. ${ }^{23}$ The amplitude of the top hat is 0.836 of the Gaussian peak and the crosswind width of the top hat is $3 \sigma_{y}$, where $\sigma_{\mathrm{y}}$ is the standard deviation (m) of the Gaussian crosswind distribution.

To obtain a finer resolution in the dose calculations of the emergency phase, each one of the 16 compass sectors is further subdivided into a number of fine grid divisions specified by the user $\left(m_{0}=3,5\right.$, or 7$)$. For a given spatial element, let $r$ be its radial interval number and $\mathrm{m}$ be the fine grid division number from the plume centerline. Then a fine spatial element may be specified using the two parameters $(r, m)$.

For the inhalation and skin exposure pathways of the emergency phase, a multistep histogram approximation of the Gaussian crosswind distribution is used for calculating the off-centerline correction factor. The boundaries of the multistep histogram are specified by the fine divisions. The outermost histogram step includes the point where the height of the Gaussian crosswind distribution falls to one-tenth of the Gaussian peak, i.e., the outermost step contains the point at which the crosswind distance is $2.15 \sigma_{\mathrm{y}}$ from the plume centerline. Figure 6 shows an example of a histogram approximation to the Gaussian crosswind distribution. In this figure, the number of fine grid divisions, $\mathrm{m}_{0}$, is 3 , the angle of each fine division is $\Delta \theta=2 \pi /\left(16^{*} 3\right)$, the outermost histogram step is 4 , and the total number of histogram steps is 7 . Let the outermost histogram step be $M$, which is derived later in this section.

The off-centerline correction factor, $J_{r m}$, of a fine spatial element $(r, m)$ is calculated as the ratio of the height of the crosswind histogram at $(r, m)$ to the height of the Gaussian peak. This correction factor $J_{\mathrm{rm}}$ is used later in the dose equations in Sections 3.2 and 3.3. The method of deriving $J_{m}$ is discussed in the following 


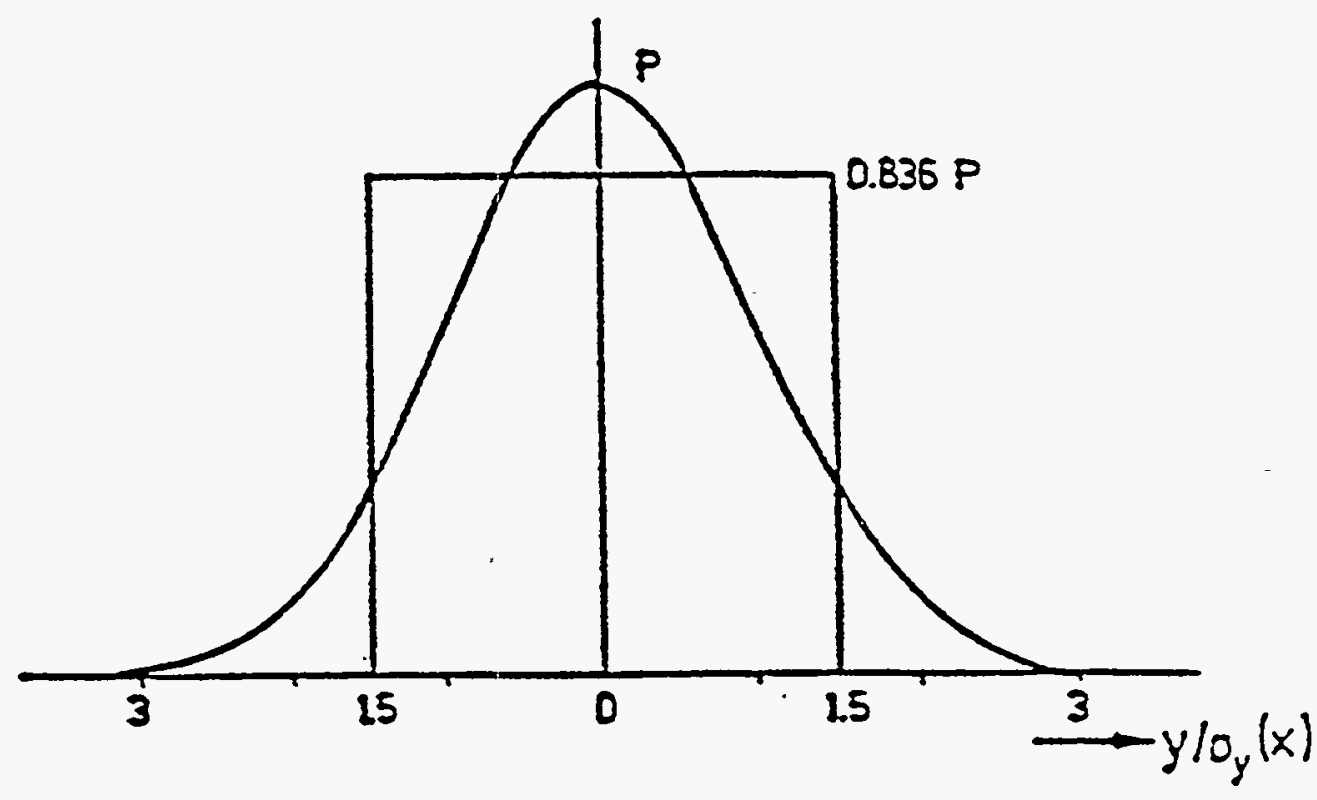

Figure 5 Top-hat approximation of the Gaussian crosswind distribution

paragraphs.

Let $\sigma_{\mathrm{yx}}$ be the standard deviation (meters) of the Gaussian crosswind distribution of a plume segment at a spatial element which has radial interval number $\mathrm{r}$. Let $\Delta \theta$ be the angle of a fine spatial element, then $\Delta \theta=2 \pi / 16 \mathrm{~m}_{0}$. Let $\theta_{\mathrm{m}}$ be the angle between the plume centerline and the outer edge of the $m$ 'th steps of the crosswind histogram, then $\theta_{\mathrm{m}}=(\mathrm{m}-1 / 2) \Delta \theta$. Furthermore, let $\mathbb{R}_{\mathrm{r}}$ be the radial distance (meters) from the midpoint of the spatial element having a radial interval number $r$ to the release point. The perpendicular distance (meters) from the plume centerline to the outer edge of the $m$ 'th step of the crosswind histogram at the midpoint of a fine spatial element $(r, m)$ is $\mathrm{R}_{\mathrm{s}} \tan \theta_{\mathrm{m}}$. Dividing this perpendicular distance by $\sigma_{\mathrm{yr}}$ gives the number of crosswind standard deviations. $J_{\mathrm{mm}}$ may then be calculated using the following equation:

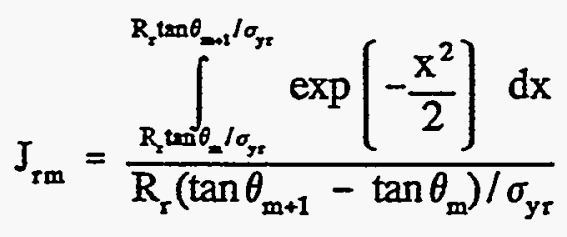

To conveniently solve Equation (3.1), the following integral is solved numerically in steps of 0.01 from $\mathrm{d}_{\sigma}=0.01$ to $\mathrm{d}_{\sigma}=3$ and the results are stored in a look-up table, which is then used to perform the integration in Equation (3.1) by linear interpolation. 


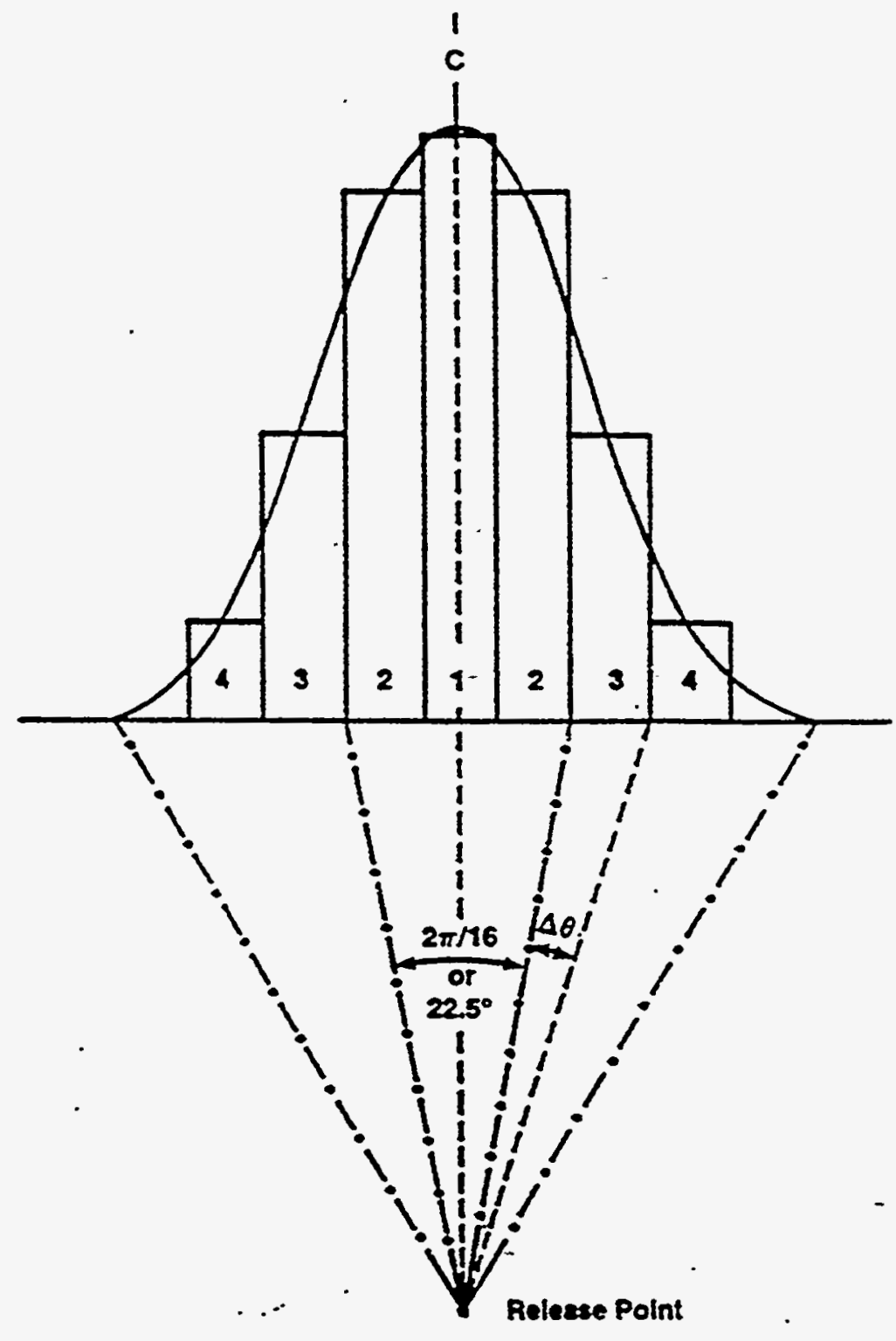

Figure 6 Approximation of a Gaussian distribution by a seven-step histogram (the number of fine grid divisions, $\mathrm{m}_{0}=3$ )

$$
A=\int_{0}^{d} \exp \left(-x^{2} / 2\right) d x
$$

The outermost histogram step, $M$, for the above off-centerline correction factor equation is determined as follows. Let $\theta_{M}$ be the angle between the outer edge of $M^{\prime}$ th histogram step and the plume centerline. Since the $M^{\prime}$ 'th histogram step is the outermost step and contains the point at which the crosswind distance is $2.15 \sigma_{\mathrm{yr}}$ from the plume centerline, $\tan \theta_{\mathrm{M}} \geq 2.15 \sigma_{\mathrm{y}} / \mathrm{R}_{\mathrm{r}}$, where $\sigma_{\mathrm{yr}}$ and $\mathrm{R}_{\mathrm{Y}}$ have been previously defined. Then $M$ is the integer portion of the following quantity: $\left(\theta_{M} / \Delta \theta+1.5\right)$. 
To simplify the subscript notation, the fine spatial element subscripts $\mathrm{r}$ and $\mathrm{m}$ are not used in the dose equations of early exposure pathways discussed in the following sections. Therefore, the off-centerline correction factor of a fine spatial element is denoted by $\mathrm{J}$ instead of $\mathrm{J}_{\mathrm{rm}}$.

\subsection{Acute Exposure Pathways and Doses}

In versions of MACCS devoted to the analysis of radiological releases, doses to various organs are calculated by summing the contributions associated with local air and ground concentrations of tracked radionuclides. All such doses are calculated in Sieverts, the standard international unit for radiological dose equivalent. The total dose to a particular organ in MACCS is the sum of the doses to that organ by various pathways.

The chemical doses calculated in CHEM_MACCS are associated with the specific health effects being analyzed. Doses associated with five different pathways are discussed in this section. The relationships between chemical doses and health effects are discussed in Chapter 5.

It should be emphasized that the doses calculated in CHEM_MACCS are those associated with the EARLY (7-day) time frame. Doses associated with the intermediate and long-term time regimes are not modeled in CHEM_MACCS. In fact, the CHRONC module of MACCS is not included in CHEM_MACCS.

\subsubsection{Plume Vapor Inhalation (PVIN) Pathway}

Doses used to estimate the number of acute health effects arising from inhalation of toxic vapors depend on the integrated air concentrations. In CHEM_MACCS, when inhalation of vapor from a plume segment passing overhead contributes to the $j$ 'th acute dose, this contribution is calculated as

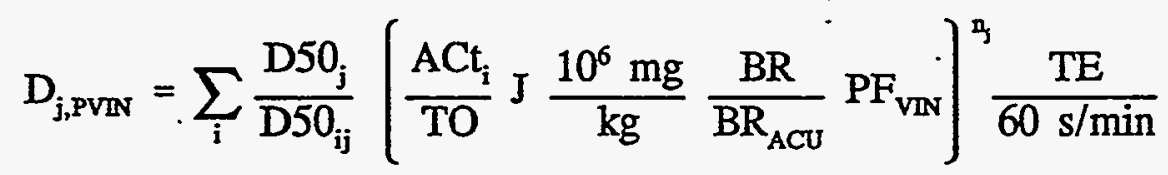

Listed alphabetically, the terms in this equation are:

$$
\begin{aligned}
& \mathrm{ACt}_{\mathrm{i}}=\text { time-integrated ground-level air concentration of chemical } \\
& \text { species } \mathrm{i} \text { under the plume centerline }\left(\mathrm{kg}-\mathrm{s} / \mathrm{m}^{3}\right) \text {, } \\
& \mathrm{BR}=\text { breathing rate }\left(\mathrm{m}^{3} / \mathrm{s}\right) \text {, } \\
& \mathrm{BR}_{\mathrm{ACv}}=\text { reference breathing rate for acute effects }\left(2.66 \mathrm{E}-04 \mathrm{~m}^{3} / \mathrm{s}\right) \text {, } \\
& D_{j, \text { PVIN }}=\text { dose due to inhalation of vapor species i directly from the } \\
& \text { passing plume ( } \mathrm{mg}-\mathrm{min} / \mathrm{m}^{3} \text { ), } \\
& \mathrm{D} 0_{\mathrm{ij}}=\text { the dose of the } \mathrm{i}^{\prime} \text { th chemical species that would produce the } \\
& j \text { 'th health effect in half of the exposed population, } \\
& \mathrm{D} \mathrm{O}_{\mathrm{j}}=\text { the dose of the reference chemical species that would } \\
& \text { produce the } j \text { 'th health effect in half of the exposed } \\
& \text { population, }
\end{aligned}
$$


$\mathrm{J}=$ off-centerline correction factor of the fine spatial element (dimensionless),

$\mathrm{n}_{\mathrm{j}}=\mathrm{a}$ user-specified power (dimensionless) to which the effective air concentration is raised for the purpose of calculating the dose,

$\mathrm{PF}_{\mathrm{VN}}=$ protection (dose reduction) factor for vapor inhalation (dimensionless),

$\mathrm{TE}=$ the exposure time of an individual in the fine spatial element (s), and

TO $=$ the time spent by the plume segment in passing over the fine spatial element (s).

Resuspended vapors, that is, vapors that become airborne as a result of the evaporation or mechanical resuspension of chemicals deposited on the ground during plume passage, also contribute to doses arising from vapor inhalation. The total dose $D_{j, V I N}$ due to inhalation of vapor species is the sum of the plume vapor inhalation dose $D_{j, P V N}$ and the resuspended vapor inhalation dose $D_{j, R V I N}$, which is discussed in Section 3.2.4.

Note that for releases of mixtures of toxic chemicals, a single effective dose $D_{j}$ is calculated by CHEM_MACCS for a user-specified reference species. The method for weighting the contributions of individual chemical species in order to obtain a single equivalent dose of the reference species is indicated in Equation (3.3) and further discussed in Chapter 5.

\subsubsection{Plume Vapor Skin (PVSK) Pathway}

Doses used to estimate the number of acute health effects arising from contact with toxic vapors are also derived from local air concentrations, $\mathrm{ACT}_{i}^{-} / \mathrm{TO}$, measured in milligrams of vapor per cubic meter. In CHEM_MACCS, the dose arising from skin contact with vapor species in a plume segment passing overhead is calculated using the equation:

$$
\mathrm{D}_{\mathrm{j}, \mathrm{PVSK}}=\sum_{\mathrm{i}} \frac{\mathrm{D} 50_{\mathrm{j}}}{\mathrm{D} 50_{\mathrm{ij}}}\left[\frac{\mathrm{ACt_{i }}}{\mathrm{TO}} \mathrm{J} \frac{10^{6} \mathrm{mg}}{\mathrm{kg}} \mathrm{PF} \mathrm{vSK}^{\mathrm{n}}\right]^{\mathrm{j}} \frac{\mathrm{TE}}{60 \mathrm{~s} / \mathrm{min}}
$$

where

$$
\begin{aligned}
& \mathrm{D}_{\mathrm{j}, \mathrm{PVSK}}=\quad \begin{array}{l}
\text { dose due to skin contact with vapor species } \mathrm{i} \text { in the plume } \\
\text { segment passing overhead (mg-min } \left./ \mathrm{m}^{3}\right),
\end{array} \\
& \mathrm{PF}_{\mathrm{VSK}}=\quad \begin{array}{l}
\text { protection (dose reduction) factor for vapor skin exposure } \\
\text { (dimensionless) }
\end{array}
\end{aligned}
$$

Note that the vapor-skin dose equation is very similar to the vapor-inhalation dose equation; however, in the vapor-skin dose equation, the breathing rate correction factor does not appear and the protection factor for skin is used in lieu of that for inhalation. 


\subsubsection{Liquid-Skin (LSK) Pathway}

The chemical mass deposited on the skin (mg/person) is used as a measure of dose in estimating the risk of certain acute health effects. Such doses arise primarily from the deposition of liquid chemical droplets from the plume directly onto the skin. Condensation of plume vapor onto the skin also contributes to the liquid-skin dose when the user specifies a positive skin-deposition velocity for the vapor form of a chemical. In CHEM_MACCS, the dose arising from deposition of chemical species onto the skin from a plume segment passing overhead is calculated using the equation:

$$
\mathrm{D}_{\mathrm{j}, \mathrm{LSK}}=\sum_{\mathrm{i}} \frac{\mathrm{D} 50_{\mathrm{j}}}{\mathrm{D} 50_{\mathrm{ij}}}\left[\frac{\mathrm{ACt_{i }}}{\mathrm{TO}} \mathrm{J} \mathrm{SDV}_{\mathrm{i}} \frac{10^{6} \mathrm{mg}}{\mathrm{kg}} \frac{1.8 \mathrm{~m}^{2}}{\text { person }} \mathrm{PF}_{\mathrm{LSK}}\right]^{\mathrm{n}_{\mathrm{j}}} \frac{\mathrm{TE}}{60 \mathrm{~s} / \mathrm{min}}
$$

where

$$
\begin{aligned}
& \mathrm{D}_{\mathrm{j}, \mathrm{LSK}}=\text { dose due to slin deposition of chemical species } \mathrm{i} \text { from a } \\
& \text { plume segment passing overhead (mg/person), } \\
& \mathrm{PF}_{\mathrm{LSK}}=\text { protection (dose reduction) factor for liquid skin contact } \\
& \text { (dimensionless), } \\
& \mathrm{SDV}_{\mathrm{i}}=\text { skin deposition velocity for } \mathrm{i} \text { 'th chemical species }(\mathrm{m} / \mathrm{s}) \text {. }
\end{aligned}
$$

\subsubsection{Resuspended Vapor Inhalation (RVIN) Pathway}

In CHEM_MACCS, chemical species deposited on the ground during plume passage may later become resuspended in the atmosphere. Resupension may result from evaporation or mechanical mechanisms. Both deposited liquids and vapors may be resuspended. The dose associated with inhalation of resuspended vapors of chemical species is calculated from the equation

$$
D_{j, R V N}=\int_{i}^{\zeta} \sum_{i} \frac{D^{2} 0_{j}}{D 50_{i j}}\left[G C_{i} J \frac{10^{6} \mathrm{mg}}{k g} \operatorname{Rw}(\tau) \frac{\mathrm{BR}}{\mathrm{BR}_{\mathrm{ACU}}} \mathrm{PF}_{\mathrm{VN}}\right]^{\mathrm{n}} \frac{\mathrm{d} \tau}{60 \mathrm{~s} / \mathrm{min}}
$$

Because the resuspension weathering function $\operatorname{Rw}(\tau)$ is taken to be an exponentially decreasing function, $\operatorname{Rw}(\tau)=R C \mathrm{e}^{-\lambda \tau}$, the preceding equation reduces to

$$
D_{j, R V N}=\sum_{i} \frac{D 50_{j}}{D 50_{i j}}\left[G C_{i} J \frac{10^{6} \mathrm{mg}}{k g} \mathrm{RC} \frac{\mathrm{BR}}{\mathrm{BR}_{\mathrm{ACU}}} \mathrm{PF}_{\mathrm{VNN}}\right]^{n_{j}} \frac{\mathrm{e}^{-n_{j} \lambda_{2} \zeta}-e^{-n_{j} \lambda_{2} \zeta}}{n_{j} \lambda_{R} 60 \mathrm{~s} / \mathrm{min}}
$$

where

$$
\begin{aligned}
& \mathrm{D}_{\mathrm{j}, \mathrm{RVN}}=\quad \text { dose due to inhalation of resuspended vapors of species } i \\
& \text { (mg- } \mathrm{min} / \mathrm{m}^{3} \text { ), } \\
& \lambda_{\mathrm{R}}=\text { resuspension weathering decay constant }\left(\mathrm{s}^{-1}\right) \text {, }
\end{aligned}
$$




$$
\begin{aligned}
& \mathrm{PF}_{\mathrm{VN}}=\text { protection (dose reduction) factor for vapor inhalation } \\
& \text { (dimensionless), } \\
& \mathrm{RC}=\text { resuspension weathering coefficient }\left(\mathrm{m}^{-1}\right) \text {, } \\
& \mathrm{t}_{1}=\text { time from plume departure to the entrance of the individual } \\
& \text { into the spatial element (s), and } \\
& t_{2}=\text { time from plume departure to the departure of the } \\
& \text { individual from the spatial element (s). }
\end{aligned}
$$

\subsubsection{Resuspended Vapor Skin (RVSK) Pathway}

The vapor-skin dose due to the resuspension of chemical species initially deposited on the ground during plume passage is directly analogous to the resuspended vapor inhalation dose discussed in the preceding section. For the vapor-skin pathway, however, the breathing rate correction factor does not apply, and the protection factor for skin rather than inhalation is applied:

$$
D_{j, R v S K}=\sum_{i} \frac{D 50_{j}}{D 50_{i j}}\left(G C_{i} J \frac{10^{6} \mathrm{mg}}{k g} R C P F_{v S K}\right]^{n} \frac{e^{-n_{j} \lambda_{R} t_{2}}-e^{-n_{j} \lambda_{R} t}}{n_{j} \lambda_{R} 60 \mathrm{~s} / \mathrm{min}}
$$

where

$$
\begin{aligned}
& \mathrm{D}_{\mathrm{i}, \mathrm{RVSK}}=\quad \begin{array}{l}
\text { dose due to skin contact with resuspended vapors of } \\
\text { chemical species } \mathrm{i}\left(\mathrm{mg}-\mathrm{min} / \mathrm{m}^{3}\right),
\end{array} \\
& \mathrm{GC}_{\mathrm{i}}=\quad \begin{array}{l}
\text { ground concentration of chemical species } \mathrm{i} \text { under the plume } \\
\text { centerline at the time that plume leaves the fine spatial } \\
\text { element }\left(\mathrm{kg}-\mathrm{s} / \mathrm{m}^{2}\right),
\end{array} \\
& \mathrm{PF}_{\mathrm{vsK}}=\quad \text { protection factor for skin exposure (dimensionless). }
\end{aligned}
$$

\subsection{Cancer-Related Doses}

The doses associated with the plume inhalation and resuspended inhalation pathways in CHEM_MACCS are expressed as continuous daily doses, which are used for computing incremental cancer risks associated with the early (7-day) exposure period. Continuous daily doses are obtained by dividing the inhaled chemical mass in milligrams by a representative body mass $(70 \mathrm{~kg})$ and a representative lifetime (70 years). The units of continuous daily dose are milligrams per kilogram-day.

\subsubsection{Continuous Daily Dose for Plume Inhalation Pathway}

The continuous daily dose associated with the plume inhalation pathway is calculated in CHEM_MACCS by the equation 


$$
D_{P C D D}=\left[\sum_{i} \frac{Q_{i}}{Q} A C t_{i}\right] \frac{T E}{T O} B R J P F_{V I N} U V_{C D D}
$$

where

$$
\begin{aligned}
& \mathrm{D}_{\mathrm{PCDD}}=\begin{array}{l}
\text { effective continuous daily dose of reference species by the } \\
\text { plume vapor inhalation pathway (mg/kg-day), }
\end{array} \\
& \mathrm{Q}_{\mathrm{i}}=\begin{array}{l}
\text { the potency factor of the } \mathrm{i} \text { 'th chemical species, } \mathrm{kg} \text {-day } / \mathrm{mg}, \\
\mathrm{Q}=\text { the potency factor of the reference chemical species, } \mathrm{kg}-
\end{array} \\
& \mathrm{UV}_{\mathrm{CDD}}=\begin{array}{l}
\text { day/mg, } \\
\text { conit conversion factor for transforming vapor }
\end{array}
\end{aligned}
$$

$$
\mathrm{UV}_{\mathrm{CDD}}=\frac{10^{6} \mathrm{mg}}{\mathrm{kg}} \frac{1}{70 \mathrm{~kg}} \frac{1}{70 \text { years }} \frac{1 \text { year }}{365.24 \text { days }}
$$

In CHEM_MACCS, one of the chemical species in the release is specified by the user as a reference. For releases that involve mixtures of chemicals, this requires only one dose to be defined for calculating the number of latent cancers. Vapor potency factors for latent cancer calculations are provided in the DOSDATA.INP file.

\subsubsection{Continuous Daily Dose for Resuspended Vapor Inhalation}

The continuous daily dose associated with the resuspended vapor inhalation pathway is calculated in CHEM_MACCS by the equation

$$
D_{R C D D}=\left[\sum_{i} \frac{Q_{i}}{Q} G C_{i}\right] \text { BR J PF } F_{V I N} U V_{C D D} R C \frac{e^{-\lambda_{R} t}-e^{-\lambda_{2} t}}{\lambda_{R}}
$$

where

$\mathrm{D}_{\mathrm{RCDD}}=\quad \begin{aligned} & \text { effective continuous daily dose of reference species by the } \\ & \text { resuspended vapor inhalation pathway (mg } / \mathrm{kg} \text {-day). }\end{aligned}$

The total continuous daily dose CDD is the sum of the dose due to the plume vapor inhalation plus that due to resuspended vapor inhalation:

$$
\mathrm{CDD}=\mathrm{D}_{\mathrm{PCDD}}+\mathrm{D}_{\mathrm{RCDD}}
$$




\section{Mitigative Actions and Dose Accumulation}

The modeling of doses to individuals following a toxic chemical release must take into account the location of the individuals during and following the accident, as well as the time period during which the doses are received. The doses received can change significantly if protective actions are taken to prevent or reduce the number of public health effects. Evacuation and sheltering are the protective actions modeled in CHEM_MACCS during the emergency phase. Since CHEM_MACCS does not model long-term exposures, the models employed in MAACS to relocate people away from hot spots after 7 days do not apply.

For a given CHEM_MACCS calculation, the user can specify up to three different emergency response strategies or scenarios and their corresponding weighting fractions. These weighting fractions are specified as fractions of the people or fractions of the time and must sum to 1.0. The EARLY module is executed and the results are presented for each of the scenarios. The weighted sum of different emergency scenarios is then calculated for each early consequence measure using the specified weighting fractions.

For a given emergency response scenario, up to three different types of protective measures can be specified by the user: evacuation, sheltering, or temporary relocation. The emergency response model of CHEM_MACCS has the capability of using an inner sheltering zone, up to three evacuation zones, and an outer sheltering zone. Figure 7 shows the schematic of different emergency response zones.

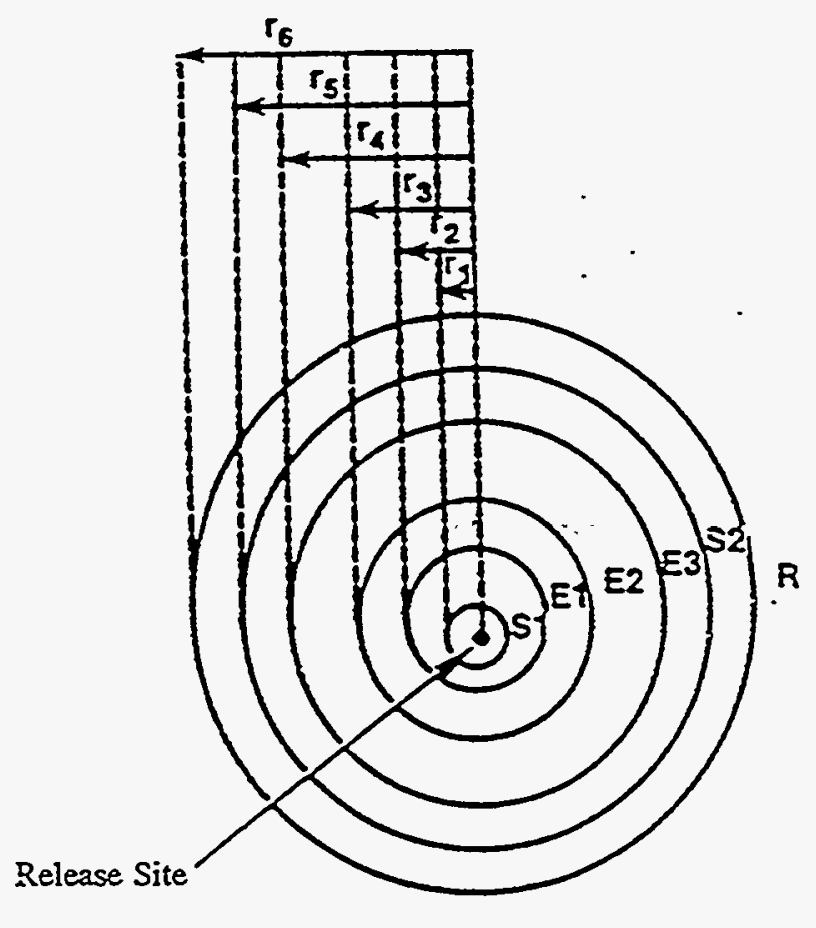

Figure 7 Schematic of emergency response zones 
The temporary relocation protective measure applies to all the spatial elements outside the evacuation or sheltering zone. The spatial elements of different protective measures shown in Figure 7 are defined as follows:

$$
\begin{aligned}
& \text { inner sheltering zone } S_{1} \text { : between } r_{1} \text { and } r_{2} \\
& \text { evacuation zone } 1, E_{1}: \text { between } r_{2} \text { and } r_{3} \\
& \text { evacuation zone } 2, E_{2}: \text { between } r_{3} \text { and } r_{4} \\
& \text { evacuation zone } 3, E_{3}: \text { between } r_{4} \text { and } r_{5} \\
& \text { outer sheltering zone } S_{2}: \text { between } r_{5} \text { and } r_{6} \\
& \text { relocation zone R: greater than } r_{6}
\end{aligned}
$$

The existence of an evacuation or sheltering zone is optional. For example, the user can define a scenario in which neither evacuation nor sheltering takes place anywhere in the region. For this case, temporary relocation applies to all spatial elements of the entire region.

A set of protection (dose reduction) factors is specified by the user for each of the three groups of people: evacuees, people taking shelter, and people continuing normal activity. The protection factors are for vapor inhalation, vapor-skin contact, and vaporliquid contact. These protection factors (less than or equal to 1.0) are used as multipliers in the dose calculations for the corresponding pathways discussed in Sections 3.2 and 3.3 to reduce the doses according to the protective measures taken at different times.

Since protection factors are used to reduce the calculated doses as linear scaling factors between 0 and 1.0 , the smaller the protection factor value, the better the protective measure. Typical relations among these protection factors are:

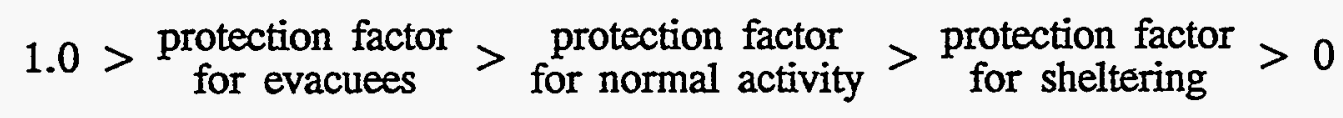

\subsection{Evacuation}

Warning times for an impending significant release of hazardous material could vary from essentially none to several hours. Depending on the accident scenario and the distance from the source, several hours might pass before the released plume would reach a particular population group, depending on the windspeed following the release. Because of this available time, evacuation is given considerable attention as a public protective measure in most current biological emergency preparedness programs in the United States. Evacuation is potentially the most effective method of avoiding exposure and can provide total protection if it is completed prior to arrival of the plume.

The CHEM_MACCS evacuation model incorporates a delay time before public movement, followed by evacuation radially away from the release point at an effective radial constant speed. Different protection factors and breathing rates can be used while individuals await evacuation (normal activity) or are being evacuated (evacuees). 
The user can specify up to three evacuation zones. Each has its own user-specified delay time before start of evacuation. The people within the designated evacuation zone are assumed to move as a group after the specified delay time. However, all evacuees of the three zones have the same evacuation speed, which is specified by the user. Evacuating people are assumed to move to a user-specified distance (e.g., 20 miles) from the accident site at which further exposure from the plume is assumed to be avoided. This model is similar to the one used in the Sandia Reactor Siting Study. ${ }^{44}$

Before the evacuees start moving, they are assumed to be carrying out normal activities. Protection factors for normal activity apply to them during this period. After they start moving, they become evacuees and the shielding factors for evacuees apply to them during evacuation.

The CHEM_MACCS plume transport model assigns the plume a finite length calculated using the assumed release duration and windspeed during the release (see Section 2.5). To simplify the treatment, the length of the cloud is assumed to remain constant following the release (i.e., the front and back of the plume travel at the same speed), and the concentration of hazardous material is assumed to be uniform over the length of the cloud. The radial position of evacuating persons, while stationary and while in transit, is compared with the positions of the front and back of the plume as a function of time to determine the period of exposure to airborne radionuclides.

CHEM_MACCS accumulates the doses for the evacuating people by adding the doses they received before they started moving and the doses received during evacuation out to a distance where they are assumed to avoid further exposure.

\subsection{Population Sheltering}

Sheltering, as used by the emergency response model, is defined as deliberate action to avoid exposure by remaining indoors and away from doors and windows during and after the passage of the plume. Normally inhabited structures offer protection from deposition onto the skin of airborne chemical species. Closing windows and/or air circulation systems can also reduce the amount of chemical species inhaled. In the model, the nonevacuating people residing within a sheitering region are exposed to chemical species using the protection factors defined for the region.

For the sheltering protective measure, the user specifies a sheltering delay time (from the alarm time) and the sheltering duration time. ${ }^{2}$ Before people go indoors and close windows, they are assumed to be carrying out normal activities and the shielding factors of normal activity apply to them during this period. After they take shelter, the shielding factors apply to them for the duration of the sheltering period. CHEM_MACCS adds the doses received before individuals take shelter to the doses they receive during the sheltering time. After the sheltering time, individuals are assumed to be removed from their spatial element and no further exposure is calculated by the EARLY module. 


\subsection{Dose Accumulation of Emergency Phase}

As far as dose accumulation during the emergency phase is concerned, the key parameter is the duration of exposure for people originally residing in each of the spatial elements in the entire region. Before calculating dose accumulation for people, CHEM_MACCS determines $t_{1}$, the time that people enter a given spatial element, and $t_{2}$, the time that people leave that spatial element. This is done for each spatial element of the entire region. The time spent by people in a given spatial element is $t_{2}-t_{1}$. This time could be spent in normal activity before evacuation or traveling time through a spatial element. CHEM_MACCS also determines the time a plume enters the spatial element, $t_{c}$, and the time the plume leaves the spatial element, $t_{0}$.

The dosimetry equations for inhalation during plume passage, resuspension inhalation after plume passage, and skin dose during plume passage are described in Chapter 3. For each of these pathways, the dose received by an individual during the exposure time between $t_{1}$ and $t_{2}$ from each spatial element that he or she entered is first calculated using the corresponding equation. If the plume never enters a spatial element, the dose from that spatial element is zero for all pathways during the emergency phase.

During the emergency phase, two types of doses are calculated for the on-grid populations: acute doses and the lifetime doses (50-year dose commitments). The acute and lifetime doses are calculated for a representative individual in a spatial element. The population dose for the on-grid populations is calculated by multiplying the individual dose by the number of people in a spatial element. The total population dose is the sum of population doses over all spatial elements.

The acute doses are used to calculate the early health effects (e.g., early fatalities and early injuries). The lifetime doses are used to calculate the delayed (latent) health effects (e.g., cancer incidences). See Chapter 5 of this report for more detail.

As discussed in Section 3.1, doses of early exposure pathways are calculated for each fine spatial element $(r, m)$, where $r$ is the radial interval number and $m$ is the fine angular division number from the plume centerline. To calculate the dose received by the population of a fine spatial element, the dose must be summed over all applicable exposure pathways, over all plume segments that cause exposures in the population group, and over all locations where the group receives exposures (only evacuees receive a dose at more than one location). Since CHEM_MACCS approximates the crosswind distribution of plume segments using a histogram, all doses also depend on the off-centerline position where they are received (see Section 3.1).

To express all of these dependencies mathematically, dose $\mathrm{k}$ to a population cohort located at a fine spatial element $(\mathrm{r}, \mathrm{m})$ must be expressed as a sum over chemicals $\mathrm{i}$, plume segments $\mathrm{n}$, and exposure pathways. Thus, dose $\mathrm{k}$ of a population cohort in a fine spatial element $(r, m)$ from plume segment $n$ is given by:

$$
\mathrm{D}_{\mathrm{krmn}}=\mathrm{DI}_{\mathrm{kmmn}}+\mathrm{DR}_{\mathrm{krmn}}
$$


where DI is the dose caused by inhalation or deposition of materials directly from the passing plume segment and $D R$ is the dose resulting from inhalation or deposition of materials resuspended from the ground (see Section 3.1).

Since it is assumed that evacuation proceeds radially outward, to calculate the total dose received by a population cohort that receives a dose at more than one location (evacuees only), the dose delivered at each radial interval number $\mathrm{r}$ where exposures are incurred must be summed:

$$
D_{\mathrm{lmm}}=\sum_{\mathbf{r}} D_{\mathrm{krmm}}
$$

Finally, the total dose $\mathrm{k}$ received by a representative cohort in a spatial element is obtained by summing the doses delivered by separate plume segments:

$$
D_{k r m}=\sum_{n} D_{k r m n}
$$


This page intentionally left blank. 


\section{Risks of Health Effects}

\subsection{Risks of Early Health Effects}

In CHEM_MACCS, probit hazard equations replace the exponential hazard curves used to estimate the risks of acute injuries and fatalities in radiological versions of MACCS. ${ }^{2}$. Before discussing the implementation of probit equations in CHEM_MACCS, relevant features of the exponential hazard curves are reviewed. The exponential form calculates the risk of the $j$-th acute effect as

$$
r_{j}=1-\exp \left[-\ln (2)\left(D_{j} / D 50_{j}\right)^{\beta_{j}}\right]
$$

where

$$
\begin{aligned}
& \mathrm{D}_{\mathrm{j}}=\begin{array}{l}
\text { the biologically effective dose causing the acute effect } \\
\text { (injury or fatality), }
\end{array} \\
& \mathrm{D} 50_{j}=\quad \begin{array}{l}
\text { the dose that would induce the effect in half the exposed } \\
\text { population, }
\end{array} \\
& \beta_{\mathrm{j}}=\text { a shape parameter. }
\end{aligned}
$$

Note that when $D_{j}=D 50_{j}, r_{j}=0.5$, as it should. Early fatalities can be caused by radiological doses to more than one organ (e.g. doses to red bone marrow, lungs, and lower intestine). In this case, the cumulative risk of early fatality is calculated as if the individual effects were independent:

$$
r_{\text {death }}=1-\prod_{k=1}^{K_{\text {mat }}}\left(1-r_{k}\right)
$$

For the exponential hazard curves, this reduces to

$$
\begin{aligned}
I_{\text {desth }} & =1-\prod_{\mathbf{k}=1}^{\mathrm{K}_{\infty+1}} \exp \left[-\ln (2)\left(\mathrm{D}_{\mathbf{k}} / \mathrm{D}_{50, \mathbf{k}}\right)^{\beta_{\mathbf{z}}}\right] \\
& =1-\exp \left[-\operatorname{In}(2) \sum_{\mathbf{k}=1}^{\hat{K}_{\infty+\infty}}\left(\mathrm{D}_{\mathbf{k}} / \mathrm{D}_{50, \mathbf{k}}\right)^{\beta_{\mathbf{z}}}\right] \\
& =1-\exp \left[-\ln (2) \mathrm{H}_{\mathrm{eff}}\right]
\end{aligned}
$$

where

$$
\mathrm{H}_{\mathrm{eff}}=\sum_{\mathbf{k}=1}^{\mathrm{K}_{\infty}}\left(\mathrm{D}_{\mathrm{k}} / \mathrm{D} 50_{\mathrm{k}}\right)^{\beta_{\mathrm{x}}}
$$


That is, $I_{\text {death }}$ can be obtained by evaluating the risk function $r=1-\exp [-\ell \operatorname{n}(2) \mathrm{H}]$ at an effective hazard $\mathrm{H}_{\text {eff }}$, which is the sum of the individual fatal effect hazards.

The risks of acute health effects due to exposures to toxic chemicals are modeled using the probit equation:

$$
r_{j}=\Phi\left(\text { probit }_{j}-5\right)
$$

where $\Phi(\mathrm{z})$ denotes the standard Gaussian cumulative distribution function

$$
\Phi(z)=\frac{1}{(2 \pi)^{1 / 2}} \int_{-\infty}^{z} \exp \left(-u^{2} / 2\right) d u=\frac{1+\operatorname{erf}(z / \sqrt{2})}{2}
$$

and

$$
\text { probit }_{j}=a_{j}+b_{j} \log _{10}\left(D_{j}\right)=5+b_{j} \log _{10}\left(D_{j} / D 50_{j}\right)
$$

The constants

$$
\begin{aligned}
& a_{j}=\text { the } y \text {-intercept, and } \\
& b_{j}=\text { the Bliss slope of the dose-response curve, }
\end{aligned}
$$

depend on the chemical and the effect being caused. As indicated by the preceding

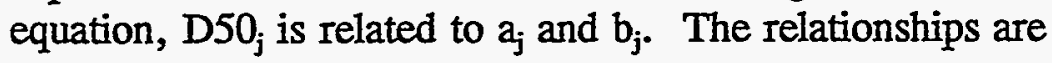

$$
\begin{gathered}
a_{j}=5-b_{j} \log _{10}\left(D 50_{j}\right) \\
D 50_{j}=10^{\left(5-z_{j}\right) / b_{j}}
\end{gathered}
$$

In CHEM_MACCS, $r_{j}$ is calculated as $\Phi\left(z_{j}\right)$ where

$$
z_{j}=\text { probit }_{j}-5=b_{j} \log _{10}\left(D_{j} / D 50_{j}\right)
$$

The total risk of early fatality is calculated by treating each of the fatal health effects as an independent event, that is, by applying Equation (5.2).

\subsection{Combining Acute Doses from Different Chemicals}

Consider two tanks ( $A$ and $B$ ), each containing a toxic chemical. The risk of a particular acute health effect due to a failure of tank $A$ alone can be written 


$$
\tau_{A}=\Phi\left[b_{A} \log _{10}\left(\frac{D_{A}}{D 50_{A}}\right)\right]
$$

Similarly, the risk of this acute effect due to failure of tank B alone can be written

$$
I_{B}=\Phi\left[b_{B} \log _{10}\left(\frac{D_{B}}{D 50_{B}}\right)\right]
$$

Now, it is tempting to say that if both containers were to fail simultaneously, the resulting risk of the health effect in question would be $r=I_{A}+I_{B}$ or perhaps $r=1$ $\left(1-r_{A}\right)\left(1-I_{B}\right)$. However, it is easy to demonstrate that both of these hypotheses are incorrect. Simply consider the case in which both tanks contain the same chemical. Then $\mathrm{D} \mathrm{O}_{\mathrm{A}}=\mathrm{D} 50_{\mathrm{B}}, \mathrm{b}_{\mathrm{A}}=\mathrm{b}_{\mathrm{B}}=\mathrm{b}$, and the appropriate risk is that obtained by inserting the sum $D_{A}+D_{B}$ in the single hazard equation. This can be written in the form

$$
I=\Phi\left[b \log _{10}\left[\frac{D_{A}}{D 50_{A}}+\frac{D_{B}}{D 50_{B}}\right]\right]
$$

This equation is extended in CHEM_MACCS to calculate risks associated with releases of mixtures of chemicals. Specifically, the mixed-release dose ratios associated with the $j$-th health effect in CHEM_MACCS is

$$
\left[\frac{D}{D 50}\right]_{j}=\sum_{i} \frac{D_{i j}}{D 50_{i j}}
$$

The Bliss slope for the mixed release is taken to be the linearly weighted value

$$
b_{j}=\frac{\sum_{i} b_{i j} \frac{D_{i j}}{D 50_{i j}}}{\sum_{i} \frac{D_{i j}}{D 50_{i j}}}
$$

The risk of health effect $\mathrm{j}$ from the mixed release is then

$$
r_{j}=\Phi\left[b_{j} \log _{10}\left[\frac{D}{D 50}\right]_{j}\right]
$$

From the release of a single species $i$, this reduces to 


$$
r_{j}=\Phi\left[b_{i j} \log _{10}\left(\frac{D_{i j}}{D 50_{i j}}\right)\right]
$$

The dose $D_{j}$ that is printed in CHEM_MACCS output for health effect $j$ is an equivalent dose of a user-specified reference species $f$. That is, setting

$$
\left[\frac{D_{j}}{D 50_{f j}}\right]^{b_{f}}=\left[\left[\frac{D}{D 50}\right]_{j}\right]^{b_{j}}
$$

gives

$$
D_{j}=D 50_{f i}\left[\left[\frac{D}{D 50}\right]_{j}\right]^{\frac{b_{j}}{b_{f}}}
$$

It should be emphasized that unless the reference species $f$ is the only one released, $D_{j}$ is an equivalent dose of reference species $f$; and $D_{j}$ is not equal to $D_{f j}$.

\subsection{Latent Cancer Risks}

In CHEM_MACCS, the additional lifetime risk of developing cancer due to inhalation doses received during the first 7 days following a chemical release is based on the linear nonthreshold dose-response relationship

$$
r_{\text {cascer }}=Q C D D=Q\left(D_{\mathrm{PCDD}}+D_{\mathrm{RCDD}}\right)
$$

where the terms are as defined with Equations (3.9) and (3.10). The risk estimate is a . measure of potential incidence (i.e., tumorigenicity and not cancer deaths). Given that the concentration weighting factors for individual chemicals in the continuous daily dose calculation are defined $\mathrm{Q} / \mathrm{Q}$, it follows that the preceding equation is equivalent to

$$
I_{\text {cancer }}=\sum_{i} Q_{i}, C D D_{i}
$$

Note that the units of $Q$ are dose reciprocal $(\mathrm{kg}$-day $/ \mathrm{mg})$. Potency factors $Q_{i}$ are often based on the upper-95\% confidence limit of the linearized dose response for animal test results judged by expert selection to be most representative of persons. In this case, any cancer risk estimate derived by the use of $Q_{i}$ will represent an upper bound. In CHEM_MACCS, the numerical values of the potency factor ratios $W_{i, j-C D D}$ are placed in column 6 of the DOSDATA.INP file table for each of the vapor species. 


\section{Comparison of CHEM_MACCS and D2PC Results}

The Personal Computer Program for Chemical Hazard Prediction (D2PC) is a computer package that estimates the downwind hazard from release of a toxic chemical. ${ }^{45}$ In D2PC, hazards are assessed in terms of accumulated dose or peak concentration resulting from an instantaneous, continuous, or time-varying release. Unlike CHEM_MACCS, D2PC is restricted to fixed weather calculations. Both D2PC and CHEM_MACCS use a Gaussian plume equation to disperse released materials in the atmosphere. The form of the Gaussian plume equation used in both codes is presented in Equation (2.11). Because CHEM_MACCS permits the user to specify the constants to be used in the equations for $\sigma_{y}$ and $\sigma_{z}$, it is possible to obtain D2PC-equivalent sigmas in CHEM_MACCS. This permits direct comparisons of CHEM_MACCS and D2PC calculations.

\subsection{CHEM_MACCS Input}

The CHEM_MACCS input used for comparisons with D2PC is discussed in the following subsections. The input is reproduced as Appendix E.1.

\subsubsection{Dispersion Equations and Constants}

The D2PC program calculates the dispersion parameters as :

$$
\begin{aligned}
& \sigma_{\mathrm{y}}(\mathrm{x})=\sigma_{\mathrm{yr}}\left(\frac{\mathrm{x}+\mathrm{B}}{\mathrm{x}_{\mathrm{yr}}}\right)^{\alpha} \quad \sigma_{\mathrm{z}}(\mathrm{x})=\sigma_{\mathrm{zx}}\left(\frac{\mathrm{x}+\mathrm{C}}{\mathrm{x}_{\mathrm{zr}}}\right]^{\beta} \\
& \mathrm{B}=\mathrm{x}_{\mathrm{yr}}\left[\frac{\sigma_{\mathrm{ys}}}{\sigma_{\mathrm{yr}}}\right)^{\frac{1}{\alpha}} \mathrm{C}=\mathrm{x}_{\mathrm{zr}}\left(\frac{\sigma_{z s}}{\sigma_{z x}}\right)^{\frac{1}{\beta}} .
\end{aligned}
$$

Table 4 gives the recommended values of the constants $\sigma_{y r}, \sigma_{z r}, x_{y r}, x_{z r}, \alpha$, and $\beta$ for open terrain. ${ }^{46} \quad B$ and $C$ are calculated from the spatial standard deviations of the initial source, $\sigma_{y s}$ and $\sigma_{z z}$. The values of $\sigma_{y s}$ and $\sigma_{z s}$ are based on the size of the sources.

CHEM_MACCS calculates dispersion parameters using a similar power-law,

$$
\begin{aligned}
& \sigma_{y i}=a_{i} x^{b_{j}} \\
& \sigma_{z i}=c_{i j} x^{d_{j}}
\end{aligned}
$$

The values of $a_{i}, b_{i}, c_{i}$, and $d_{i}$ for the $i$ 'th stability class are user specified in the ATMOS input file. In CHEM_MACCS, $\sigma_{\mathrm{y}}$ can be scaled to correct for horizontal plume meander and $\sigma_{z}$ can be scaled to account for effects of surface roughness. These corrections have the effect of increasing the plume volume, thereby decreasing plume concentration. In 
Table 4 Recommended values for D2PC sigmas $\left(\mathrm{x}_{\mathrm{yr}}=\mathrm{x}_{\mathrm{zx}}=100 \mathrm{~m}\right)$

\begin{tabular}{|c|c|c|c|c|c|}
\hline \multirow{2}{*}{$\begin{array}{c}\text { Stability } \\
\text { Category }\end{array}$} & \multicolumn{2}{|c|}{$\sigma_{\mathrm{yz}}(\mathrm{m})$} & \multirow{2}{*}{$\sigma_{\mathrm{zx}}(\mathrm{m})$} & $\alpha$ & $\beta$ \\
\cline { 2 - 3 } & Instantaneous & Continuous & & & \\
\hline A & 9.0 & 27.0 & 14.0 & 1.0 & 1.4 \\
B & 6.33 & 19.0 & 11.0 & 1.0 & 1.0 \\
C & 4.8 & 12.5 & 7.5 & 1.0 & 0.9 \\
D & 4.0 & 8.0 & 4.5 & 0.9 & 0.85 \\
E & 3.0 & 6.0 & 3.5 & 0.8 & 0.8 \\
F & 2.0 & 4.0 & 2.5 & 0.7 & 0.75 \\
\hline
\end{tabular}

addition, the initial values of the dispersion parameters are determined by the size of the building (height and width) from which the material is released.

Since both CHEM_MACCS and D2PC use power law equations to model the dispersion parameters, matching the values of the initial sigmas and the leading and exponential terms used in the $\sigma$ calculations should produce comparable results. To obtain D2PC-equivalent sigmas for CHEM_MACCS, the constants $a_{i}, b_{i}, c_{i}$, and $d_{i}$ were assigned values as follows:

$$
\begin{array}{ll}
\mathrm{a}_{\mathrm{i}}=\frac{\sigma_{\mathrm{yr}}}{\mathrm{x}_{\mathrm{yr}}{ }^{\alpha}} & \mathrm{c}_{\mathrm{i}}=\frac{\sigma_{\mathrm{zx}}}{\mathrm{x}_{\mathrm{zx}}{ }^{\beta}} \\
\mathrm{b}_{\mathrm{i}}=\alpha & \mathrm{d}_{\mathrm{i}}=\beta
\end{array}
$$

The resulting constants, which are used to calculate the D2PC-equivalent sigmas, are shown in Table 5.

Table 5 Constants for CHEM-MACCS to obtain D2PC-equivalent sigmas

\begin{tabular}{|c|c|c|c|c|c|}
\hline $\begin{array}{c}\text { Stability } \\
\text { Class }\end{array}$ & $\begin{array}{c}\text { Class } \\
\text { Subscript, } \mathrm{i}\end{array}$ & $\mathrm{a}_{\mathrm{i}}$ & $\mathrm{b}_{\mathrm{i}}$ & $\mathrm{c}_{\mathrm{i}}$ & $\mathrm{d}_{\mathrm{i}}$ \\
\hline $\mathrm{A}$ & 1 & 0.2700 & 1.0 & 0.0222 & 1.40 \\
$\mathrm{~B}$ & 2 & 0.1900 & 1.0 & 0.1100 & 1.00 \\
$\mathrm{C}$ & 3 & 0.1250 & 1.0 & 0.1189 & 0.90 \\
$\mathrm{D}$ & 4 & 0.1268 & 0.9 & 0.0898 & 0.85 \\
$\mathrm{E}$ & 5 & 0.1507 & 0.8 & 0.0879 & 0.80 \\
$\mathrm{~F}$ & 6 & 0.1592 & 0.7 & 0.0791 & 0.75 \\
\hline
\end{tabular}


The linear CHEM_MACCS scaling factors for $\sigma_{\mathrm{y}}$ and $\sigma_{\mathrm{z}}$ that account for surface roughness were "turned off" by assigning

DPYSCALEO01 $=1$

DPZSCALE001 $=1$.

\subsubsection{Plume Meander Data}

EXPFAC is a linear expansion factor for $\sigma_{y}$ that is used to calculate ground concentrations. EXPFAC is a ratio raised to the power XPFAC1001 or XPFAC2001. The CHEM MACCS scaling factors that account for plume meander were "turned off" by setting the relevant program variables to their minimum values as follows

PMXPFAC1001 $=0.01$

PMXPFAC2001 $=0.01$.

\subsubsection{Release Description Data}

CHEM-MACCS can handle multiple plume segments of varying sizes, durations, and energy content. One plume segment was specified and assigned an energy content of 3.35 watts (essentially zero) to eliminate plume rise. The following values were assigned:

$$
\begin{aligned}
& \text { RDPLHTTE001 }=0, \text { release height }(\mathrm{m}) \\
& \text { RDPLUDUR001 }=3600, \text { release duration (seconds) } \\
& \text { RDCORINV001 }=1.22 \mathrm{E}+6, \text { size of source }(\mathrm{mg}) \\
& \text { RDCORSCA001 }=1.0, \text { fraction of source released }
\end{aligned}
$$

\subsubsection{Meteorological Data}

Three test runs were made for stability class A, windspeed $2 \mathrm{~m} / \mathrm{s}$; stability class $D$, windspeed $2.5 \mathrm{~m} / \mathrm{s}$; and stability class $E$, windspeed $4 \mathrm{~m} / \mathrm{s}$. The relevant ATMOS input variables are as follows:

M1METCOD001 $=4$, selects constant condition

M2BNDMXH001 $=220$, height of mixing layer (m)

M2IBDSTB001 $=1$, stability A (4, stability D; 5 , stability E)

M2BNDRAN001 $=0$, rainfall rate $(\mathrm{mm} / \mathrm{hr})$

M2BNDWND001 $=2$, wind speed $(\mathrm{m} / \mathrm{s})(2.5 \mathrm{~m} / \mathrm{s}, 4 \mathrm{~m} / \mathrm{s})$

\subsection{D2PC Input Data}

$\mathrm{D} 2 \mathrm{PC}$ is a PC-based interactive program. The input to the program was printed from the screen to a file and is included as Appendix E.2. The location was not defined so that the mixing layer height could be specified $(220 \mathrm{~m})$. The weather data were the same as for the CHEM_MACCS runs, stability class A, windspeed $2 \mathrm{~m} / \mathrm{s}$; stability class $D$, windspeed $2.5 \mathrm{~m} / \mathrm{s}$; and stability class $\mathrm{E}$, windspeed $4 \mathrm{~m} / \mathrm{s}$. A "nonagent" semicontinuous release of total amount $1.22 \mathrm{E}+6 \mathrm{mg}$ was specified. The duration of the 
release was set at $60 \mathrm{~min}(3600 \mathrm{~s})$. The variable NDI was set equal to 8 after the "all other input" prompt so that doses of interest could be entered. (In D2PC, the user specifies a dose of interest and the program calculates the distance to that dose.) CHEM_MACCS was run prior to running the D2PC program so that the ground level, centerline doses calculated by CHEM_MACCS could be input into the D2PC program.

\subsection{Results}

Based on the input data discussed in the last section, the ground-level, centerline (downwind) doses at the midpoints of the intervals were calculated by the CHEMMACCS. D2PC was then used to calculate the distance at which the doses are equal to those calculated in the CHEM-MACCS runs. Tables 6, 7, and 8 show the results of the calculations for the three different weather conditions. The D2PC-calculated distances for each of the CHEM_MACCS calculated doses are in very close agreement with the midpoint distances specified in the CHEM_MACCS input. This verifies that CHEM_MACCS and D2PC dispersion calculations agree very well.

Table 6 Comparison of CHEM-MACCS and D2PC results for stability class A (windspeed $2 \mathrm{~m} / \mathrm{s}$, mixing layer height $220 \mathrm{~m}$, semicontinuous release, $1.22 \mathrm{E} 6 \mathrm{mg}, 60 \mathrm{~min}$ )

\begin{tabular}{|c|c|c|}
\hline & $\begin{array}{c}\text { CHEM-MACCS results } \\
\text { using D2PC-equivalent } \\
\text { constants in } \sigma \text { formulas }\end{array}$ & $\begin{array}{c}\text { D2PC } \\
\text { results }\end{array}$ \\
\hline $\begin{array}{c}\text { Distance: midpoint } \\
\text { between specified } \\
\text { radii on grid (m) }\end{array}$ & $\begin{array}{c}\text { Ground-level } \\
\text { centerline dose } \\
\left.\text { (mg-min } / \mathrm{m}^{3}\right)\end{array}$ & $\begin{array}{c}\text { Distance to } \\
\text { specified dose } \\
\text { (m) }\end{array}$ \\
\hline 100 & 8.0 & 103 \\
350 & 0.433 & 347 \\
750 & 0.0970 & 715 \\
1500 & 0.0483, & 1414 \\
3500 & 0.0207 & 3299 \\
7500 & 0.00966 & 7068 \\
15000 & 0.00483 & 14137 \\
35000 & 0.00207 & 32986 \\
\hline
\end{tabular}


Table 7 Comparison of CHEM-MACCS and D2PC results for stability class D (windspeed $2.5 \mathrm{~m} / \mathrm{s}$, mixing layer height $220 \mathrm{~m}$, semicontinuous release, $1.22 \mathrm{E} 6 \mathrm{mg}, 60 \mathrm{~min}$ )

\begin{tabular}{|c|c|c|}
\hline & $\begin{array}{c}\text { CHEM-MACCS results } \\
\text { using D2PC-equivalent } \\
\text { constants in } \sigma \text { formulas }\end{array}$ & $\begin{array}{c}\text { D2PC } \\
\text { results }\end{array}$ \\
\hline $\begin{array}{c}\text { Distance: midpoint } \\
\text { between specified } \\
\text { radii on grid (m) }\end{array}$ & $\begin{array}{c}\text { Ground-level } \\
\text { centerline dose } \\
\left.\text { (mg-min } / \mathrm{m}^{3}\right)\end{array}$ & $\begin{array}{c}\text { Distance to } \\
\text { specified dose } \\
\text { (m) }\end{array}$ \\
\hline 100 & 70.8 & 101 \\
350 & 8.33 & 343 \\
750 & 2.22 & 730 \\
1500 & 0.664 & 1454 \\
3500 & 0.151 & 3390 \\
7500 & 0.0435 & 7187 \\
15000 & 0.0215 & 14149 \\
35000 & 0.0100 & 32916 \\
\hline
\end{tabular}

Table 8 Comparison of CHEM-MACCS and D2PC results for stability class E (windspeed $4 \mathrm{~m} / \mathrm{s}$, mixing layer height $220 \mathrm{~m}$, semicontinuous release, $1.22 \mathrm{E} 6 \mathrm{mg}, 60 \mathrm{~min}$ )

\begin{tabular}{|c|c|c|}
\hline & $\begin{array}{c}\text { CHEM-MACCS results } \\
\text { using D2PC-equivalent } \\
\text { constants in } \sigma \text { formulas }\end{array}$ & $\begin{array}{c}\text { D2PC } \\
\text { results }\end{array}$ \\
\hline $\begin{array}{c}\text { Distance: midpoint } \\
\text { between specified } \\
\text { radii on grid (m) }\end{array}$ & $\begin{array}{c}\text { Ground-level, } \\
\text { centerline dose } \\
\left.\text { (mg-min } / \mathrm{m}^{3}\right)\end{array}$ & $\begin{array}{c}\text { Distance to } \\
\text { specified dose } \\
(\mathrm{m})\end{array}$ \\
\hline 100 & 75.8 & 100 \\
350 & 10.8 & 338 \\
750 & 3.22 & 720 \\
1500 & 1.07 & 1433 \\
3500 & 0.276 & 3343 \\
7500 & 0.0817 & 7157 \\
15000 & 0.0309 & 14814 \\
35000 & 0.0150 & 31917 \\
\hline
\end{tabular}


This page intentionally left blank. 


\section{Summary and Recommendations}

\subsection{MACCS and CHEM_MACCS}

MACCS was developed at Sandia National Laboratories to perform probabilistic calculations of the potential offsite consequences of the atmospheric release of radioactive material in reactor accidents. MACCS was extensively checked and tested during its development, and has been continuously maintained by Sandia National Laboratories under contract with the NRC. MACCS has been used in many probabilistic risk assessments, including the NUREG-1150 study and is widely used by Department of Energy facilities for safety analysis reports. ${ }^{10}$

Science Applications International Corporation sponsored the development of CHEM MACCS from MACCS to provide the capability for calculating the probabilistic offsite consequences of the accidental atmospheric release of hazardous chemicals. CHEM-MACCS provides the following capabilities, which are not generally available in combination in chemical release consequence codes:

a. Statistical weather sampling of site-specific data $(8,760$ hourly data points per year)

b. Population dose and health effect risk calculations based on site-specific population data.

c. Health effects calculations that include the consideration of potential site-specific mitigative actions, including evacuation, shielding, and relocation activities.

d. Modeling of multiple-release segments.

\subsection{CHEM_MACCS Models}

CHEM MACCS only includes two modules: ATMOS and EARLY. The CHRONC module of MACCS is not included. CHEM_MACCS does not account for decay or reactions of hazardous chemicals.

In MACCS, doses to various organs are calculated by summing the contributions associated with local air and ground concentrations of tracked radioactive nuclides. The total dose.to a particular organ is the sum of the doses to that organ by various pathways. In CHEM_MACCS, the chemical doses are associated with five different exposure pathways, and CHEM_MACCS combines the doses from different chemicals by linearly weighing the relevant air or skin concentrations of the different chemicals in proportion to their capability to cause the health effect in question.

MACCS calculates the risk of a specified health effect based on cumulative hazard. The cumulative hazard is a function of the normalized biologically effective dose. In CHEM_MACCS, the health-effect risks are based on probit equations or potency 
factors as specified by the users. The risk values represent the percent of the population that could be expected to suffer the specified effect as a result of exposure to a toxic substance.

CHEM_MACCS introduces a new model for specifying the start time and the end time of the accident. The user can specify the accident-initiatiation interval; for example, forcing any accident to be initiated during workhours (e.g. from 8:00 a.m. to 5:00 p.m.).

\subsection{CHEM_MACCS Input and Output}

Input for the ATMOS module is unchanged from MACCS. As for MACCS, the following outputs can be obtained from the EARLY module:

1. cases of a given health effect

2. early fatality radius

3. population exceeding a specified dose

4. average individual risk

5. population dose

6. centerline dose versus distance

7. centerline risk versus distance

8. population-weighted risk

CHEM_MACCS introduces two new output options: (9) the maximum distance at which a user-specified level of early injury risk is exceeded, and (10) the area of land contaminated in excess of a user-specified level. The input and output files are modified according to changes in the models.

\subsection{CHEM_MACCS Testing}

In the process of developing CHEM_MACCS from MACCS, several verification strategies were used. First, the development process was undertaken in stages with an example problem used at the end of each stage to verify that the coding tasks had been completed successfully. The test problem used for a particular stage varied according to the nature of the tasks undertaken, but the focus was on maintaining a working interim version of the code at all times. In this way, one could always go back to the working version from the previous stage if a particular coding effort became too difficult to debug. A line-by-line check of all coding used to implement CHEM_MACCS models was done. A few minor changes were made to CHEM_MACCS as a result of this lineby-line inspection. Finally, a set of verification calculations, including the D2PC comparison calculations discussed in Chapter 6, was undertaken. A more detailed discussion of verification activities is included as Appendix F.

Three test problems are used to compare CHEM_MACCS with D2PC. In these three test problems, a source term is given and the weather conditions are changed. For each test problem, CHEM_MACCS calculated the downwind centerline doses at the midpoints of the intervals, and then D2PC calculated the distances relevant to those doses. From the results discussed in Chapter 6, the downwind centerline distances from 
the source for the given doses are in very close agreement.

\subsection{Recommendations}

CHEM_MACCS performs probabilistic calculations of potential offsite consequences of the atmospheric release of four chemicals: nerve agents GA, GB, VX, and the blister agent $\mathrm{HD}$ based on data described in Appendix G. Additional hazardous chemicals should be included in the future. Since the doses are dependent on the air and ground concentrations of the hazardous chemicals, an exact description of the properties of the chemicals is very important. Future activities should investigate other deposition models, especially models for dense gases, which are very different from the dispersion models in MACCS. 
This page intentionally left blank. 


\section{References}

1. U.S. News and World Report, Vol. 117, No. 22, p. 14, December 5, 1994.

2. D. I. Chanin, J. L. Sprung, L. T. Ritchie, and H-N Jow, MELCOR Accident Consequence Code System (MACCS) User's Guide, NUREG/CR-4691, SAND861562, Vol.1, Sandia National Laboratories, Albuquerque, NM, February 1990.

3. H-N Jow, J. L. Sprung, J. A. Rollstin, L. T. Ritchie, and D. I. Chanin, MELCOR Accident Consequence Code System (MACCS), Model Description, NUREG/CR-4691, SAND86-1562, Vol. 2, Sandia National Laboratories, Albuquerque, NM, February 1990.

4. J.A. Rollstin, D.I. Chanin, and H-N Jow, MELCOR Accident Consequence Code System (MACCS), Programmer's Reference Manual, NUREG/CR-4691, Vol. 3, SAND861562, Sandia National Laboratories, Albuquerque, NM, February 1990.

5. D. I. Chanin, J. Rollstin, J. Foster, L. Miller, MACCS Version 1.5.11.1: A Maintenance Release of the Code, NUREG/CR-6059, SAND92-2146, Sandia National Laboratories, Albuquerque, NM, October 1993.

6. C. A. Dobbe, E. R. Carlson, N. H. Marshall, E. S. Marwil, and J. E. Tolli, Quality Asurance and Verification of the MACCS Code, Version 1.5, Idaho National Engineering Laboratory, Idaho Falls, ID, NUREG/CR-5376, February 1990.

7. L. Neymotin, Comparison of MACCS Users Calculations for the International Comparison Exercise on Probabilistic Accident Consequence Assessment Codes, NUREG/CR-6053, BNL-NUREG-52380, Brookhaven National Laboratory, Upton, NY, April 1994.

8. U. Tveten, Review of the Chronic Exposure Pathway Models in MACCS and Several Other. Well-Known Probabilistic Risk Assessment Models, Institut for Energiteknikk, Norway, June 1990.

9. V. L. Peterson, R. W. Patlovany, and G. A. Ennis, Comparisons Between MACCS and GENII, NSTR-017-92, EG\&G Rocky Flats, Inc., Golden, CO, October 1992.

10. U.S. Nuclear Regulatory Commission, Severe Accident Risks: An Assessment for Five U.S. Nuclear Power Plants, NUREG-1150, NRC, Washington, DC, December 1990.

11. D. J. Hall and R. A. Waters, Further Experiments on a Buoyant Emission from a Building, Warren Spring Laboratory, Stevenage, England, LR 567 (PA), 1986. 
12. G. A. Briggs, Plume Rise Predictions, Environmental Research Laboratories, U.S. Department of Commerce, National Oceanic and Atmospheric Administration, Wahington DC, 1975.

13. S. R. Hanna et al., Handbook on Atmospheric Diffusion, DOE/TIC-11223, U.S. Department of Energy, Washington, DC, 1982.

14. USNRC, PRA Procedures Guide, Environmental Transport and Consequence Analysis, NUREG/CR-2300, Vol. 2, Chapter 9, U.S. Nuclear Regulatory Commission, Washington, DC, 1983.

15. Commission of the European Communities, Radiation Protection: Methods for Assessing the Off-Site Radiological Consequences of Nuclear Accidents, EUR 10243 en, Commission of the European Communities, Luxemburg, 1986.

16. S. K. Koa, "Theories of Atmospheric Transport and Diffusion," in Atmospheric Sciences and Power Production, D. Randerson, ed., U.S. Department of Energy, Washington, DC, DOE/TIC-27601; available from National Technical Information Service, Springfield, VA, 1984.

17. G. C. Holzworth, Mixing Heights, Wind Speeds, and Potential for Urban Air Pollution Throughout the Contiguous United States, AP- 101, U.S. Environmental Protection Agency, Office of Air Programs, Research Triangle Park, NC, 1972.

18. D. B. Turner, Workbook of Atmospheric Dispersion Estimates, PSH-999-AP-26, U.S. Department of Health, Education, and Welfare, Washington, DC, 1970.

19. H. D. Brenk and K. J. Vogt, "The Calculation of Wet Deposition from Radioactive Plumes," Nuclear Safety, Vol. 22, p. 362 (1981).

20. G. A. Sehmel, "Deposition and Resuspension," in Atmospheric Sciences and Power Production, D. Randerson, ed., U. S. Department of Energy, Washington, DC, DOE/TIC-27601; available from National Technical Information Service, Springfield, VA, 1984.

21. USNRC, Reactor Safety Study, Calculation of Reactor Accident Consequences, WASH-1400, Appendix VI, U.S. Nuclear Regulatory Commission, Washington, DC, 1975.

22. J. C. Helton et al., "Contamination of Surface-Water Bodies after Reactor Accidents by the Erosion of Atmospherically Deposited Radionuclides," Health Physics, Vol. 48, p. 757 (1985).

23. L. T. Ritchie et al., CRAC2 Model Description, NUREG/CR-2552, SAND82-0342, Sandia National Laboratories, Albuquerque, NM, 1984. 
24. G. Briggs, "Lift Off of Buoyant Gas Initially on the Ground," ADTL Contribution File No 87 (Draft), Nov. 1973. Air Resources Atmospheric Turbulence and Diffusion Laboratory NOAA, Oak Ridge, TN.

25. R. C. West, Ed., Handbook of Chemistry and Physics, 53rd ed., p. F-169, Chemical Rubber Co., Cleveland, OH, 1972.

26. USNRC, Regulatory Guide 1.23, U.S. Nuclear Regulatory Commission, Washington, DC, 1972.

27. G. A. Briggs, "Plume Rise and Buoyancy Effects," in Atmospheric Science and Power Production, D. Randerson, ed., DOE/TIC-27601; available from National Technical Information Service, Springfield, VA, 1984.

28. D. A. Haugen, Ed., "Project Prairie Grass: A Field Program in Diffusion," Geophysical Research Papers, No. 59, Vol. III, Report AFCRC-TR-58-235, Air Force Cambridge Research Center, 1959.

29. F. Pasquill, "The Estimation of the Dispersion of Windborne Material," Meteorological Magazine, Vol. 90, p. 33 (1961).

30. F. Gifford, "Atmospheric Dispersion Models for Environmental Pollution Applications," in Lectures on Air Pollution and Environmental Impact Analysis, D. A. Haugen, ed, p. 42, AMS, Boston, MA, 1975.

31. F. A. Gifford, "A Review of Turbulent Diffusion Typing Schemes," Nuclear Safety, Vol. 17, p. 68, 1976.

32. J. Tadmor and Y. Gur, "Analytical Expressions for the Vertical and Lateral Dispersion Coefficients in Atmospheric Diffusion, "Atmospheric Environment, Vol. 3, p. 688, 1969.

33. R. A. Dobbins, Atmospheric Motion and Air Pollution. Wiley, New York, 1979.

34. H. Lettau, "Note on Aerodynamic Roughnesss-Parameter Estimation on the Basis of Roughness-Element Description," Joumal of Applied Meteorology, Vol. 8, p. 826, 1969.

35. D. Randerson, "Atmospheric Boundary Layer," in Atmospheric Science and Power Production, D. Randerson, ed., U.S. Department of Energy, Washington, DC, DOE/TIC-27601; available from National Technical Information Service, Springfield, VA, 1984.

36. "American Meteorological Society Workshop on Stability Classification Schemes and Sigma Curves-Summary and Recommendations," Bulletin of the American Meteorological Society, Vol. 58, 1977. 
37. S. F. Mueller and L. M. Reisinger, "Measured Plume Width Versus Sampling Time: A Look Beyond 10 Kilometers," Atmospheric Environment, Vol. 20, p. 895 (1986).

38. M. Hino "Maximum Ground-Level Concentration and Sampling Time," Atmospheric Environment, Vol. 2, p. 149 (1968).

39. R. R. Draxler, "Diffusion and Transport Experiments," Atmospheric Sciences and Power Production, D. Randerson, ed., U.S. Department of Energy, Washington, DC, DOE/TIC-27601; available from National Technical Information Service, Springfield, VA, 1984.

40. A. C. Chamberlain, Aspects of Travel and Deposition of Aerosol and Vapour Clouds, British Report AERE-HP/R 1261, Atomic Energy Research Establishment, Harwell, United Kingdom, 1953.

41. R. P. Hosker, Jr., "Estimates of Dry Deposition and Plume Depletion over Forests and Grassland," in Physical Behavior of Radioactive Contaminants in the Atmosphere, Symposium Proceedings, International Atomic Energy Agency, Vienna, 1974.

42. Stig Karlsson, Dry Deposition - A Literature Review, Studsvik Energiteknik AB, Studsvik/NW-82/242, Sweden, 1982.

43. J. A. Jones, The Uncertainty in Dispersion Estimates Obtained From the Working Group Models, NRPB-R199, National Radiological Protection Board, Chilton, Didcot, Oxon, United Kingdom, 1986.

44. D. C. Aldrich et al., Technical Guidance for Siting Criteria Development, NUREG/CR-2239, SAND81-1549, Sandia National Laboratories, Albuquerque, NM, 1981.

45. C. Glenvil Whitacre, Joseph H. Griner III, Michael M. Myirski, and Dale W. Sloop, Personal Computer Program for Chemical Hazard Prediction (D2PC), CRDEC-TR-87021, Studies and Analysis Office, Aberdeen Proving Grounds, MD, 1987.

46. C. Glenvil. Whitacre, Downwind Hazard from Bursting M23 Land Mine, ARCSL-TR-79023, Chemical Research, Development and Engineering Center, Aberdeen Proving Ground, MD, April 1979. 


\section{APPENDIX A}

\section{User Input Processing}

\section{A.1 Introduction}

In some cases, the user may not need to exercise all of the code's features. For instance, if all that is needed is a calculation of air and ground concentrations as a function of distance, only the ATMOS module need be exercised and EARLY can be skipped.

The OUTPUT module generates complementary cumulative distribution functions (ccdfs) of the results generated by EARLY when the user requests that those results be produced as described in Appendix $C$. This is done for both single and multiple weather trial runs. The results that can be produced are defined in Sections C.12 to C.21.

A ccdf is generated internally for all of the user requested consequence measures. For each of these ccdfs, the code always produces a one-line summary describing various aspects of the distribution function that is written to the List Output File.

For any subset of the results, the user can, in addition, cause the code to print out the entire ccdf table. This feature is under user control and is described in each of the sections where the user requests the production of the individual consequence measures.

The results from the OUTPUT module are presented individually for each emergency response strategy, and also as a weighted sum of the combined results. Consequences calculated by EARLY (such as cancer cases) are summed together in the overall weighted sum. In addition, the weighting fractions associated with the individual emergency response scenarios of EARLY (up to three are allowed) are automatically combined according to the values of "fraction of the people" or "fraction of the time" specified on the EARLY User Input File (WTFRAC).

There is no provision for specifying each accident's expected rate of occurrence (accident frequency). All consequence measures calculated by CHEM_MACCS are conditional on the occurrence of a particular accident. For directions on the interpretation of the consequence values presented by the OUTPUT module, please refer to Appendix D.

CHEM_MACCS can handle multiple source terms in a single run. When more than one source term is specified, the results for each are presented on the output listing in the order of their appearance on the ATMOS User Input File. The OUTPUT module will print a description of all the results for each source term before going on to the next source term. The code is currently dimensioned to handle up to 60 source terms at a time. 
When the user has requested that multiple weather trials be performed according to weather category sampling (Section B.16), the user has the capability of having the OUTPUT module present a table of relative contribution from each weather category sampling bin (Section B.2). This enables the user to see what type of weather conditions contribute the most to the mean consequence value of each result on the output listing. These tables can only be produced if weather category sampling is being used (METCOD =2). This feature is especially useful now that the user has some control over the bins defining weather category since it can provide a means of "fine-tuning" the bin definitions. Section B.11 provides additional information on the interpretation of the relative contribution tables.

Parameters defining both the rain intensity and rain distance bins are specified on the ATMOS User Input File as described in Section B.16. In contrast to the categorization of rain events, which is under the user's control as defined in Section B.16, the initial condition bins for stability class and windspeed remain hard wired into the code with no provision for easy modification by the user.

\section{A.2 Input Format}

The format of the input files was designed to maximize their readability. The input processor allows comments to be freely interspersed with the data, which allows the input files to documented internally.

Certain restrictions on the format of the input data files are necessary. The input data files consist of a sequence of card images, with each card allowed a length of up to 100 characters. The sequence of cards is delimited by a special terminator record. A terminator record is a card with a period (.) in column one. Each card in a file is either a comment card or a data card. Comment cards are denoted by an asterisk $\left(^{*}\right)$ in column one. Data cards are required to begin in column one with an eleven-character record identifier consisting of eight or nine alpha- numeric identification characters followed by two or three numeric characters which are used for sequencing. An example of a record identifier is RIATNAM1001.

The input processor begins by sorting all the data cards in the file according to the collating sequence of their record identifiers. If more than one data card has the same record identifier, the last card encountered is used and all earlier cards with the same identifier are ignored. Cards may appear in any order. The ordering of data cards is only important for cards sharing the same record identifier.

Multiple source terms for ATMOS and multiple emergency response strategies for EARLY can be specified by the addition of "change cards" positioned at the end of the ATMOS and EARLY user input files. Each set of change cards is separated from the rest by a period (.) in Column 1. ATMOS allows up to 59 sets of source term change cards and EARLY allows up to two sets of emergency response change cards. Each change card set must specify a new text field to describe the source term or scenario and at least one previously defined input value must be redefined in each set of change cards. If the user tries to override a previously defined input variable that is not 
in the data blocks for source term (Section B.11) or emergency response (Sections C.6 and C.7), those cards will be ignored by the program.

Every card appearing in the source term and emergency response change card sets must have been previously supplied in the base case input for that file. That is, when the change cards are being processed, there is no capability of adding new cards to the input file database. The change cards can only be used to replace previously defined data items in the base case input. The change card processor simply replaces a previously defined data card with the new card which has the same record identifier. If a set of change cards contains data cards with record identifiers which have not been defined in the base case input, it is possible that spurious results will be generated. All of the CHEM_MACCS User Input Files must end with a period in column one.

Data required by CHEM_MACCS can be of four different types: logical, character, integer, or real. Logical values are represented as either .TRUE. or .FALSE. as defined in the ANSI FORTRAN 77 standard. ${ }^{1}$ Character values can be any ASCII string delimited by apostrophes ('). If the string has no embedded blanks, the apostrophes are optional provided that the string is not interpreted to be a data item of another type. Integer values can be preceded with a minus sign or an optional plus sign but they cannot have a decimal point. Real values can be written either as an optionally signed number with a decimal point (FORTRAN $F$ format) or else in exponential notation (FORTRAN E format) as defined in the ANSI standard.

The determination of the type of each item encountered is performed as follows: the code first determines if the item is of type logical, if it isn't, it checks to see if it is of type integer, if it isn't, it checks to see if it is of type real. Any item that is neither logical, integer nor real is considered to be of type character.

More than one item can be placed on a card. Multiple items on a card are delimited either by a comma or a blank. The presence of additional blanks between items has no effect on how the items are interpreted. Multiple commas between items are not allowed.

The input processor will not convert the type of the values that it encounters from real to integer or vice versa. If an integer value is required by one of the program modules, then an integer value must be supplied by the user. If a decimal point appears in what should be an integer value, an error flag will be set and execution will be terminated upon completion of the input processing phase. Likewise, the absence of a decimal point in what should be a real value will be interpreted as an input error and cause the error flag to be set.

In addition to checking that each data item encountered is of the correct type, the input processor determines the validity of the numeric input parameters (both integer and real) by checking whether they fall within the specified range. The allowable range of each datum is listed in Appendices B and $\mathrm{C}$ of this report. Character string values have a length between specified limits; these limits are also listed here. If a value is encountered that falls outside the required range, the error flag will be set and a 
diagnostic message issued to the output file. This message lists the minimum and maximum allowable values for the item in order to facilitate the debugging process.

If any input errors are detected, the code will usually attempt to process as much as possible of the subsequent input. In many instances though, the occurrence of an input error will cause an immediate termination of the program's execution.

A data element consists of either a single value (scalar) or a set of values which are all of the same type (array). Within a user input file for a particular program module, the data that it requires has been broken up into groups of functionally related quantities. These data groups are described in separate sections of the following chapters.

The record identifier used for scalar values is always of the same form. The first two characters are a mnemonic for the data group to which it belongs. For example, the mnemonic for the Run Identification Data is RI. The next six characters of the record identifier are the name of the FORTRAN variable within the program that is used to store the value. The record identifier is always 001 . Only the first item found on the card for a scalar quantity will be processed. Anything to the right of the data item is ignored by the input processor. This allows the use of descriptive comments alongside of the data item. This feature should only be used for scalar values.

Arrays are found in the input files in two different forms. The first method is similar to the approach used for scalars. A starting record identifier is constructed the same as above: a two-letter mnemonic, followed by the six-letter variable name, followed by 001 . More than one value can appear on the data card separated by blanks or a comma. You may place as many values on the card as will fit into the 100 columns. Successive cards with ascending sequence numbers are processed until all the required data items have been supplied. The record identifiers of the succeeding cards differ from the starting record identifier only in their last three digits.

The second method used for arrays is more structured. Several arrays of the same length whose values are related appear as columns across the page. The arrays can be of different type but they all share the same record identifier. The name of a program variable is not used to construct the record identifier since more than one variable receives its value from this "data block." Instead of reading array values from left to right in rows, the arrays are read from top to bottom in columns.

All of the input parameters used by the program modules will be described in this report. Unless specified otherwise, all of these parameters are required to be supplied. Each input parameter is described in a stylized block of text which presents the following information: (1) the FORTRAN variable name used in the code, (2) the type of the data item (integer, real, logical or character), (3) an indication of whether only a single value is required (scalar), or multiple values are required (array), (4) minimum and maximum allowable values (or lengths), (5) a statement describing the variable, and (6) an example of the variable's use. 


\section{A.3 Sample Problems}

Three sample problems are provided to illustrate the use of CHEM_MACCS. These will be referred to as problems VX_A, VX_C, and MIX_C. Due to the length of the output listings it is impractical to include them all in this document's printed text. All of the input and output files for the sample problems are available in machine-readable form.

Throughout the body of this document, records similar to those supplied in the sample problem input files are used to illustrate the appearance of the data in those files. It is the responsibility of each CHEM_MACCS user to ensure the appropriateness of all data in the CHEM_MACCS input files which they prepare.

Problem VX_A shows how CHEM_MACCS can be used to address a variety of needs including regulatory studies and conventional parametric variation sensitivity studies. It illustrates how CHEM_MACCS can automatically loop on source terms and emergency response assumptions in a single run of the code. The source term only contains one hazardous chemical species, VX. The weather category bin sampling method is used in this problem to estimate the distribution of consequences which could result from an accident if the time of the accident's occurrence is unknown. Automatic looping is illustrated as follows: the ATMOS User Input File for Problem VX_A causes results to be calculated for two hypothetical source terms, and the EARLY User Input File specifies two different emergency response scenarios ( 95 percent evacuation and 5 percent nonevacuation). An additional sheltering case is presented for illustration.

Problem VX_C illustrates how CHEM_MACCS can be used to examine one of the weather sequences which was selected in the weather sampling used for Problem VX_A. This type of examination is usually done when some type of unusual result is noticed on the output listing and the user wishes to determine if the calculations are being properly performed. By going back to the first part of the listing, we can see that this trial began at day $=157$ and hour $=10$ on the weather data file.

By themselves, the numbers printed on the debug output listing may be hard to interpret, but they could be helpful if it is necessary to examine intermediate steps in the calculations.

In Problem MIX_C, the code is set up to run with constant meteorology; D-stability, $5 \mathrm{~m} / \mathrm{s}$ wind speed, no rain. A uniform population distribution of 50 people $/ \mathrm{km}^{2}$ is used for the calculations and therefore there is no need for a Site Data File. The source term contains all chemical species GA, GB, HD, and VX. There is no evacuation. In this run, only the standard presentation of results from the OUTPUT module appears on the output listing.

Once CHEEM_MACCS is installed on a computer system, the user can verify that all is well by running the sample problems and comparing the resulting output files with those on the distribution magnetic tape which were generated on a DOS system. Since CHEM_MACCS is intended to be portable to any computer, the numeric output values 
by and large should be identical or very close to identical. The only significant deviation might lie in the probabilities of non-zero and peak value consequences since the precision of arithmetic and the handling of underflow will vary on different computer systems.

If the user is working with a DOS system, the CHEM_MACCS command file included on the installation tape may be used to execute the three sample problems as follows after the input files have been loaded and the program compiled and linked. The sample problems can be executed by typing the following three command lines.

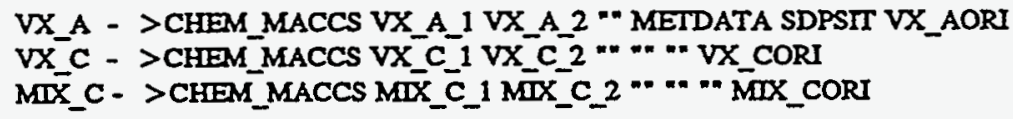

The output from these sample problems will be found in the three files: VX_AORI.OUT, VX_CORI.OUT, and MIX_CORI.OUT. Sample Problems VX_C and MIX__C do not require a meteorological data file (METDATA) or a Site Data File (SDPSIT), since they use constant weather conditions and a uniform population density.

\section{References}

1.

"American National Standard Programming Language FORTRAN," ANSI X3.9-1978, American National Standards Institute, 1430 Broadway, New York, NY 10018. 


\section{APPENDIX B}

\section{ATMOS Input File}

\section{B.1 Introduction}

The ATMOS program calculates the dispersion and deposition of material released to the atmosphere as a function of downwind distance. The phenomena that ATMOS treats are (1) building wake effects, (2) buoyant plume rise, (3) plume dispersion during transport, and (4) wet and dry deposition..$^{1,2,3}$ It utilizes a Gaussian plume model with Pasquill-Gifford dispersion parameters.

At the midpoint of each spatial interval along the transport path, air and ground concentrations for all the chemical species are calculated as well as miscellaneous information about plume size, height, and transport timing. This data is stored in common blocks which are used later by the EARLY module of CHEM_MACCS.

Transport and deposition in ATMOS are treated with a one-dimensional model. Concentration values are calculated only for the plume centerline. There is no calculation in ATMOS of off-centerline concentrations. The adjustment for off-axis location is handled in the EARLY module.

CHEM_MACCS incorporates a database of eight chemical species. In any single CHEM_MACĆS run, the user can define a list of up to 8 chemical species (including vapor and liquid).

Several different options for specifying weather conditions are available to the user. These include two weather sampling options: (1) eategory bin sampling and (2) strictly random sampling; as well as three different methods of specifying a single weather trial: (1) constant weather conditions, (2) fixed start time in the weather file, and (3) user-supplied 120-hr weather sequence.

It is up to the user to specify the various parameters needed for these calculations. There are no default values. All of this information is supplied through the user input file to ATMOS and all of the input parameters are described in this appendix.

Downwind transport, dispersion, and deposition are treated in the ATMOS module of the MACCS code. In addition to the values of the parameters implemented in its phenomenological models, the ATMOS module also requires that the nature of the release and the dimensions of the computational grid be specified as input. Given these data, ATMOS models plume liftoff and plume rise, the capping of plume rise and of vertical plume expansion by inversion layers, downwind transport of the plume, horizontal and vertical dispersion of the plume, plume depletion by wet and dry deposition, and radioactive decay, and calculates the centerline air and ground concentrations that these processes produce on the computational grid. 
Most of the models implemented in the ATMOS module use weather conditions as input data. Either constant or variable weather data can be used. Variable data are specified as a sequence of hourly values of windspeed, atmospheric stability class, and amount of precipitation, which begins at a time specified by the user or selected by the weather categorization and sampling algorithm embedded in the code. If variable data are used to model a release that is divided into plume segments, the user must designate one of the segments as risk dominant, whereupon ATMOS automatically causes the release of that segment to coincide with the start time of (first hour of data in) the variable sequence of weather conditions. The user must also select a representative weather point for each plume segment, which determines the weather conditions that will be used to calculate all transport processes except wet deposition.

\section{B.2 Run Identification (RI) Data}

In order to identify the computer run that is being performed, the user is required to supply a text field that will be printed on all of the list output produced for this run by CHEM_MACCS. All of the CHEM_MACCS programs obtain the current date and time from the computer operating system so it is not necessary to include information of that type. In addition to this text field, a text field describing the source term is supplied separately in the Release Description Data Block, which defines the source term.

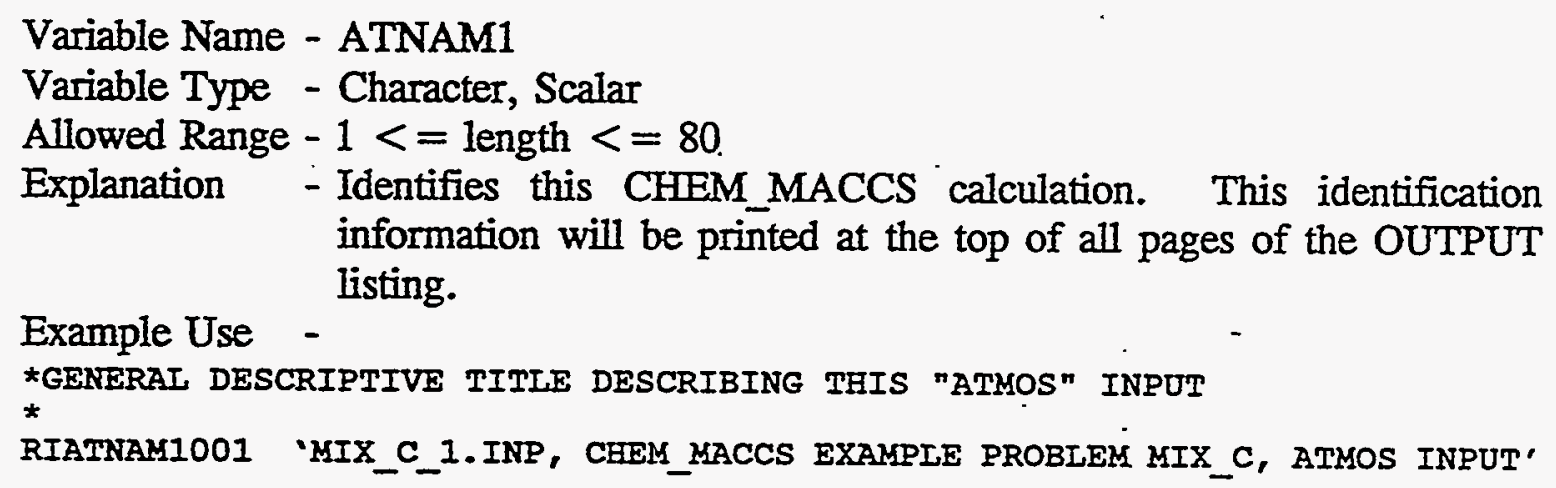
information will be printed at the top of all pages of the OUTPUT listing.

Example Use -

*GENERAI DESCRIPTIVE TITLE DESCRIBING THIS "ATMOS" INPUT

*

RIATNAM1001 'MIX_C_1.INP, CHEM_MACCS EXAMPLE PROBLEM MIX_C, ATMOS INPUT'

\section{B.3 Geometry (GE) Data}

A polar grid coordinate system is used in CHEM_MACCS to represent the region surrounding the plant. The plant itself is always located at the centerpoint of the coordinate system $(r=0)$. The data in this section define the grid spacing between spatial elements in the radial direction. All of the consequence calculations performed by CHEM_MACCS are stored on the basis of the radial spacing defined here. For example, air and ground concentrations are calculated to be representative of the entire length of the spatial element (not just its centerpoint).

Variable Name - NUMRAD

Variable Type - Integer, Scalar

Allowed Range $-2<=$ value $<=35$ 
Explanation - Number of radial spatial intervals defined in the model. This quantity defines the polar coordinate spatial grid which will be used by the two program modules: ATMOS and EARLY. If a Site Data File is being used, the value supplied here must match exactly the value supplied

Example Use on that file as variable NSPDTS.

* NUMBER of RADIAI SPATIAI ELEMENTS

*

GENUMRADO01 26

Variable Name - SPAEND

Variable Type - Real, Array

Allowed Range $-0.001<=$ value $<=9999$. $(\mathrm{km})$

Explanation - Distance in kilometers to the endpoints of the spatial intervals. If a Site Data File is being used, the values supplied here must be within 10 percent of the corresponding parameter-values supplied on that file for the array SPDSTS.

Note to user - The spacing between adjacent spatial intervals should be at least $0.1 \mathrm{~km}$.

Example Use

*

*SPATIAL ENDPOINT DISTANCES IN RILOMETERS

*

$\begin{array}{lrrrrr}\text { GESPAENDOO1 } & 0.16 & 0.52 & 1.21 & 1.61 & 2.13 \\ \text { GESPAENDOO2 } & 3.22 & 4.02 & 4.83 & 5.63 & 8.05 \\ \text { GESPAENDO03 } & 11.27 & 16.09 & 20.92 & 25.75 & 32.19 \\ \text { GESPAENDOO4 } & 40.23 & 48.28 & 64.37 & 80.47 & 112.65 \\ \text { GESPAENDO05 } & 160.93 & 241.14 & 321.87 & 563.27 & 804.67 \\ \text { GESPAENDOO6 } & 1609.34 & & & & \end{array}$

Note to user - Elements in the array must be separated by blanks or a comma. The number of items per card is left to the user's discretion. The sequence numbers of the record identifiers must be in ascending order.

\section{B.4 Chemical Species (IS) Data}

This section defines the chemical species to be modeled and associated modeling parameters. Vapor and liquid forms of the same chemical must be treated as different chemical species.

Variable Name - NUMISO

Variable Type - Integer, Scalar

Allowed Range $-1<=$ value $<=10$

Explanation - Number of chemical species defined in the model. Several input routines use this value to determine the number of values to be supplied. The concentration weights file (DOSDATA.INP) must have weights for each of the chemical species defined here. 


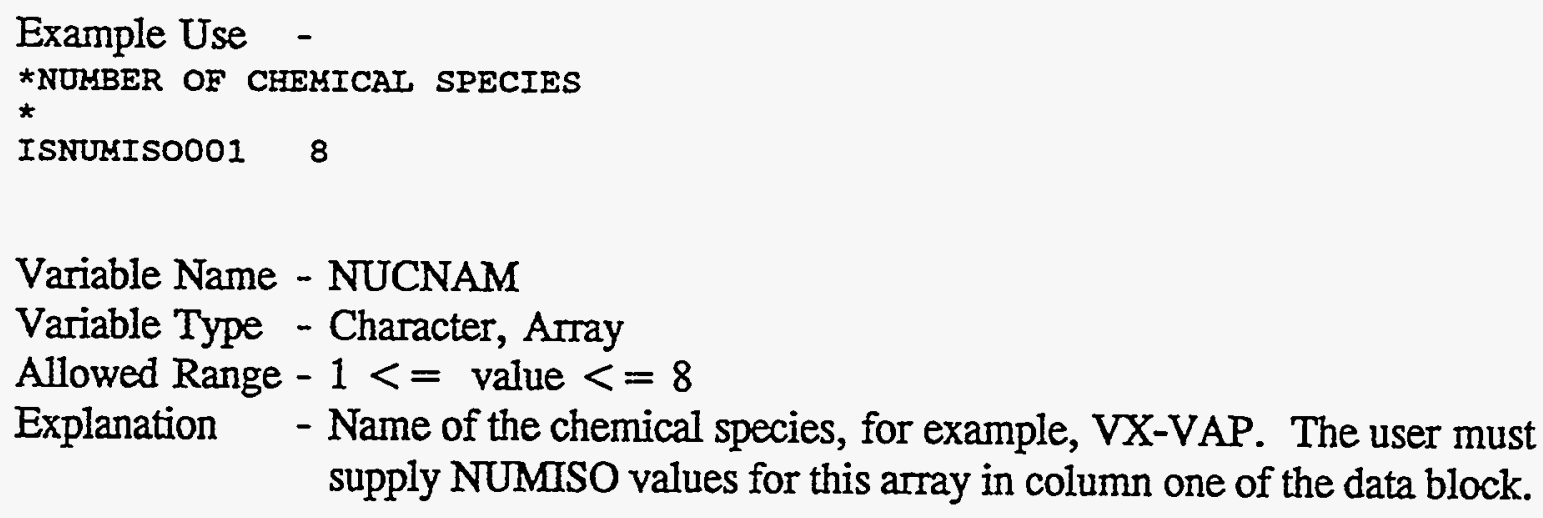

Variable Name - NUCNAM

Variable Type - Character, Array

Allowed Range $-1<=$ value $<=8$

Explanation - Name of the chemical species, for example, VX-VAP. The user must supply NUMISO values for this array in column one of the data block.

Variable Name - WETDEP

Variable Type - Logical, Array

Allowed Range - .TRUE. or .FALSE.

Explanation - Logical flag that indicates for each chemical species whether it is subject to wet deposition. The user must supply NUMISO values in column two of the data block.

Variable Name - DRYDEP

Variable Type - Logical, Array

Allowed Range - .TRUE. or .FALSE.

Explanation - Logical flag that indicates for each chemical species whether it is subject to dry deposition. The user must supply NUMUSO values in column three of the data block.

Variable Name - SDV

Variable Type - Real, Array

Allowed Range $-0 .<=$ value $<=10 .(\mathrm{m} / \mathrm{s})$

Explanation - The skin deposition velocity $(\mathrm{m} / \mathrm{s})$ for a chemical species. The user must supply NUMISO values in column four of the data block.

Example Use of NUCNAM, WETDEP, DRYDEP, and SDV:

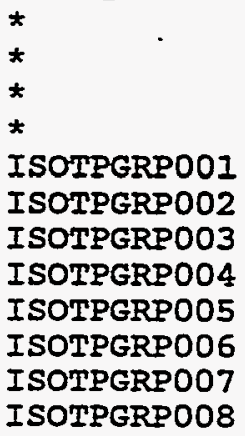

B.5 Wet Deposition Data

\begin{tabular}{|c|c|c|c|}
\hline $\begin{array}{l}\text { CHEMICAI } \\
\text { SPECIES }\end{array}$ & WETDEP & DRYDEP & $\begin{array}{l}\text { SRIN DEP. } \\
\text { VELOCITY }\end{array}$ \\
\hline $\begin{array}{l}\text { GA-IIQ } \\
\text { GB-IIQ } \\
\text { VX-IIQ } \\
\text { HD-IIQ } \\
\text { GA-VAP } \\
\text { GB-VAP } \\
\text { VX-VAP } \\
\text { GD-VAP }\end{array}$ & $\begin{array}{l}\text {-TRUE } \\
\text {-TRUE } \\
\text {-TRUE. } \\
\text { - TRUE . } \\
\text { - TRUE . } \\
\text {-TRUE . } \\
\text {-TRUE . } \\
\text { TRUE . }\end{array}$ & $\begin{array}{l}\text { - TRUE } \\
\text { - TRUE. } \\
\text { - TRUE. } \\
\text { - TRUE . } \\
\text { - TRUE. } \\
\text { - TRUE. } \\
\text { - TRUE. } \\
\text {-TRUE. }\end{array}$ & $\begin{array}{l}0.01 \\
0.01 \\
0.01 \\
0.01 \\
0.01 \\
0.01 \\
0.01 \\
0.01\end{array}$ \\
\hline
\end{tabular}

Incorporated in chemical species data.

Incorporated in chemical species data. 


\section{B.7 Dispersion Parameter (DP) Data}

The Gaussian plume model of atmospheric dispersion uses spatially dependent dispersion parameters, sigma-y and sigma-z. Sigma-y and sigma-z are functions of the form:

$$
\begin{aligned}
& \text { sigma-y }=\text { CYSIGA }(X) * * \text { CYSIGB }, \text { and } \\
& \text { sigma-z }=\text { CZSIGA }(X) * * \text { CZSIGB, }
\end{aligned}
$$

where sigma- $y$, sigma- $z$, and $X$ (the downwind distance from the source), are all in meters.

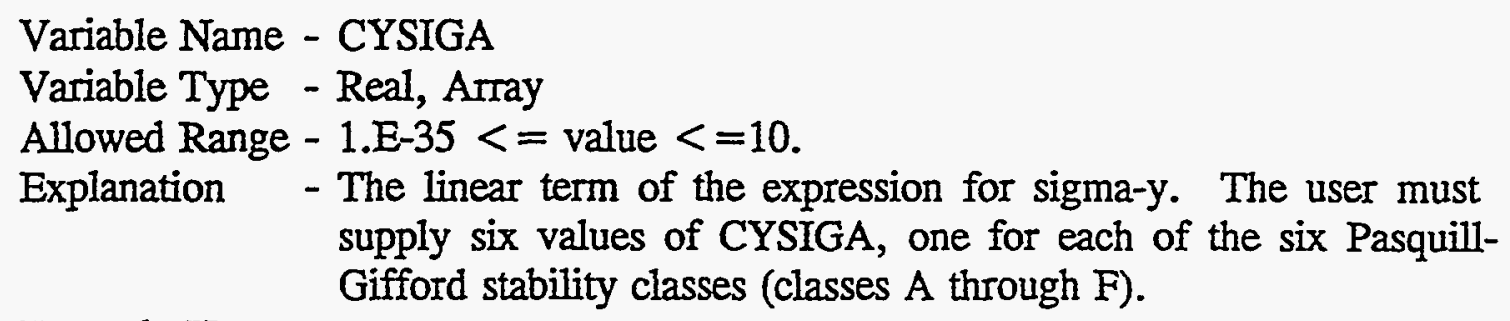
supply six values of CYSIGB, one for each of the six Pasquill-

Example Use Gifford stability classes (classes A through F). supply six values of CZSIGA, one for each of the six PasquillGifford stability classes (classes A through F).

Example Use -

*IINEAR TERM OF THE EXPRESSION FOR SIGMA-Z, 6 STABIIITY CLASSES

$*$

$\begin{array}{llllllll}* \text { STABILITY CLASS: } & A & B & C & D & E & F\end{array}$

$\begin{array}{lllllll}\text { DPCZSIGA00I } & 2.5 E-4 & 1.9 E-3 & 0.2 & 0.3 & 0.4 & 0.2\end{array}$ 


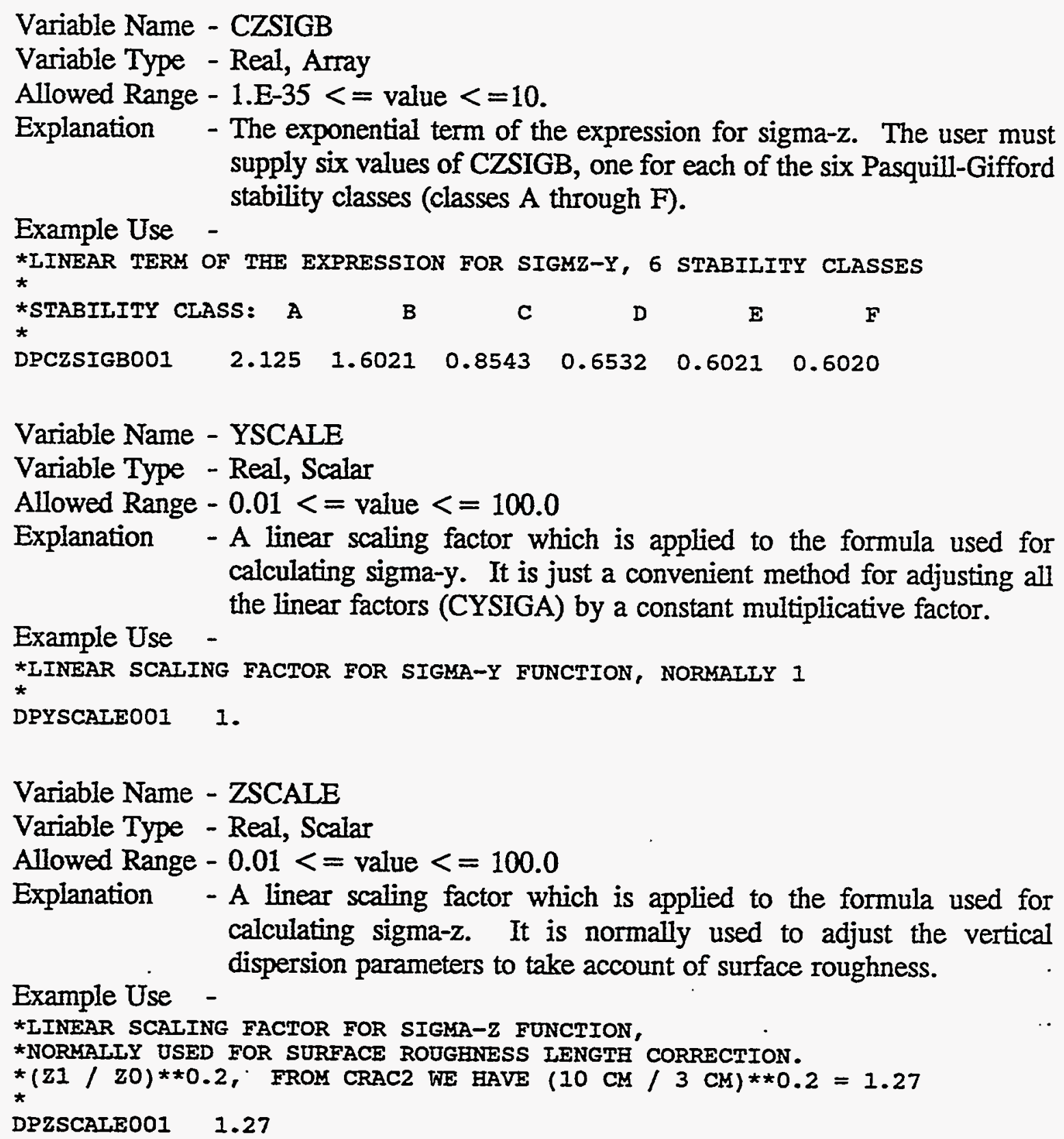
supply six values of CZSIGB, one for each of the six Pasquill-Gifford calculating sigma-y. It is just a convenient method for adjusting all calculating sigma-z. It is normally used to adjust the vertical

\section{B.8 Plume Meander (PM) Data}

In order to account for the effects of meander during transport of the plume, an expansion factor, EXPFAC, is calculated which serves to widen the plumes in the crosswind direction. It acts as a linear factor on sigma-y during the calculation of $\chi / Q$, but it does not affect the rate of growth of sigma-y. A two-part function is used. The expansion factors used for different plume segments are independent of each other. If the release duration of the plume segment is less than or equal to BRKPNT, then the following formula will be used,

$$
\text { EXPFAC }=(\text { plume-segment-release-duration / TMMBAS }) * * \text { XPFAC1. }
$$


If the plume segment duration exceeds BRKPNT, then a different factor is used for the exponent of the function,

$$
\text { EXPFAC }=\text { (plume-segment-release-duration } / \text { TIMBAS) } * * \text { XPFAC2. }
$$

In both expressions, the duration of the plume segment is limited to $10 \mathrm{hr}$. A warning is printed on the output listing if the user specifies a release duration exceeding $10 \mathrm{hr}$.

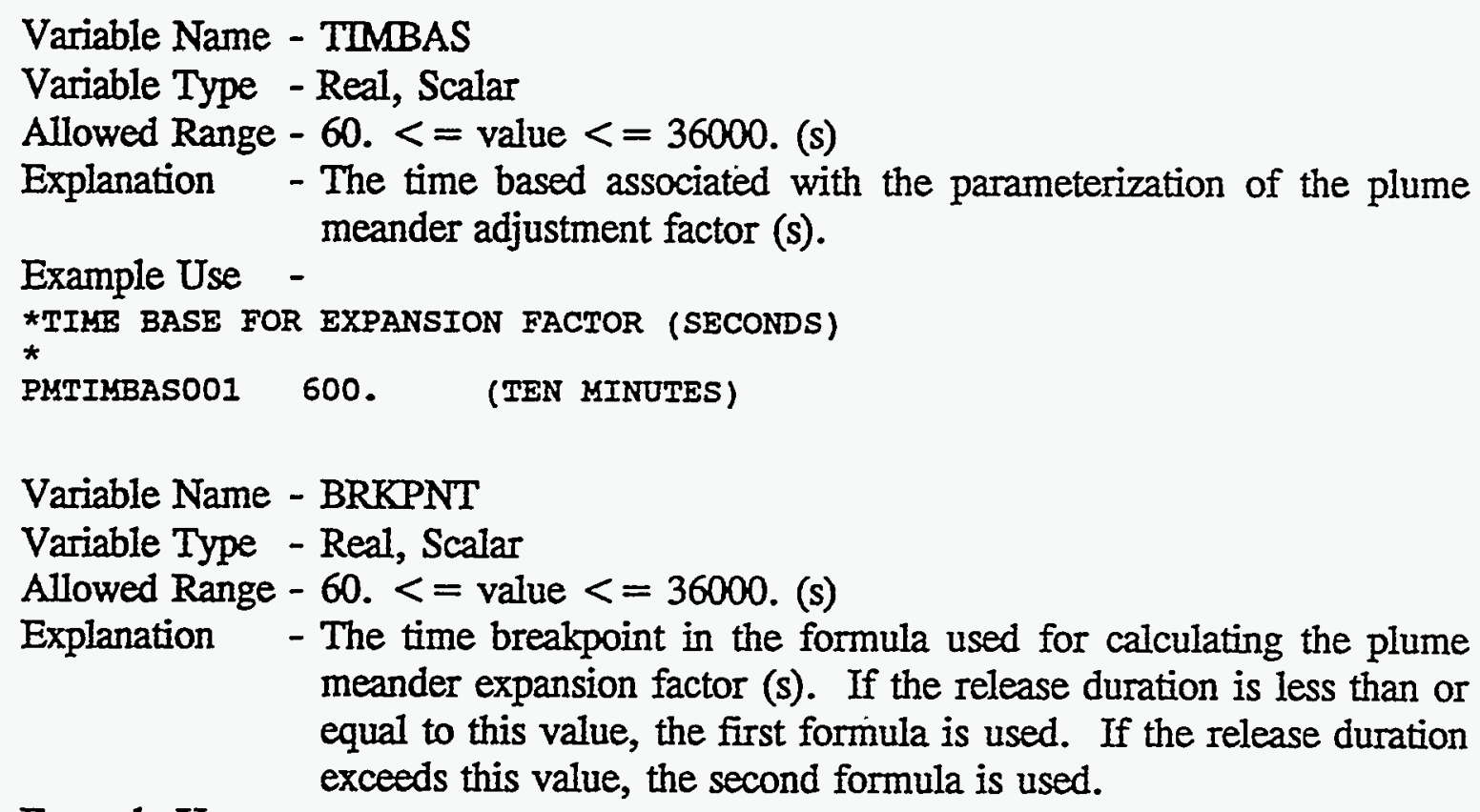
meander adjustment factor (s).

Example Use -

*TIME BASE FOR EXPANSION FACTOR (SECONDS)

*

PMTIMBASO01 600. (TEN MINUTES)

Variable Name - BRKPNT

Variable Type - Real, Scalar

Allowed Range -60 . $<=$ value $<=36000$. (s)

Explanation - The time breakpoint in the formula used for calculating the plume meander expansion factor (s). If the release duration is less than or equal to this value, the first formula is used. If the release duration exceeds this value, the second formula is used.

Example Use -

*BREARPOINT FOR FORMULA CEANGE (SECONDS)

PMBRKPNTO01 3600 .

Variable Name - XPFAC1

Variable Type - Real, Scalar

Allowed Range $-0.01<=$ value $<=1$.

Explanation - The exponential factor used in calculating the plume meander expansion factor for releases having durations that are less than or

Example Use equal to BRKPNT.

*EXPONENTIAI EXPANSION FACTOR NURBER 1

PMXPFAC1001 0.2

Variable Name - XPFAC2

Variable Type - Real, Scalar

Allowed Range $-0.01<=$ value $<=1$.

Explanation - The exponential factor used in calculating the plume meander expansion factor for releases having durations that are greater than BRKPNT. 
Example Use -

*EXPONENTIAL EXPANSION FACTOR NUMBER 2

*

PMXPFAC1001 0.25

\section{B.9 Plume Rise (PR) Data}

The CHEM_MACCS plume rise model is the same as that incorporated into MACCS. There are three basic components of the MACCS plume rise model: (1) entrainment of buoyant plumes in building wake, (2) plume rise under unstable and neutral conditions (classes A through D), and (3) plume rise under stable conditions (classes E to F). These component models are described in the MACCS Model Description. ${ }^{2}$ The individual numeric coefficients utilized by these models are hard wired into the code with no provision for their modification by the user.

While it is not possible for the user to vary the individual coefficients utilized by the three components of the plume rise model, it is possible to modify their end result by the specification of linear scaling factors that are described in this section.

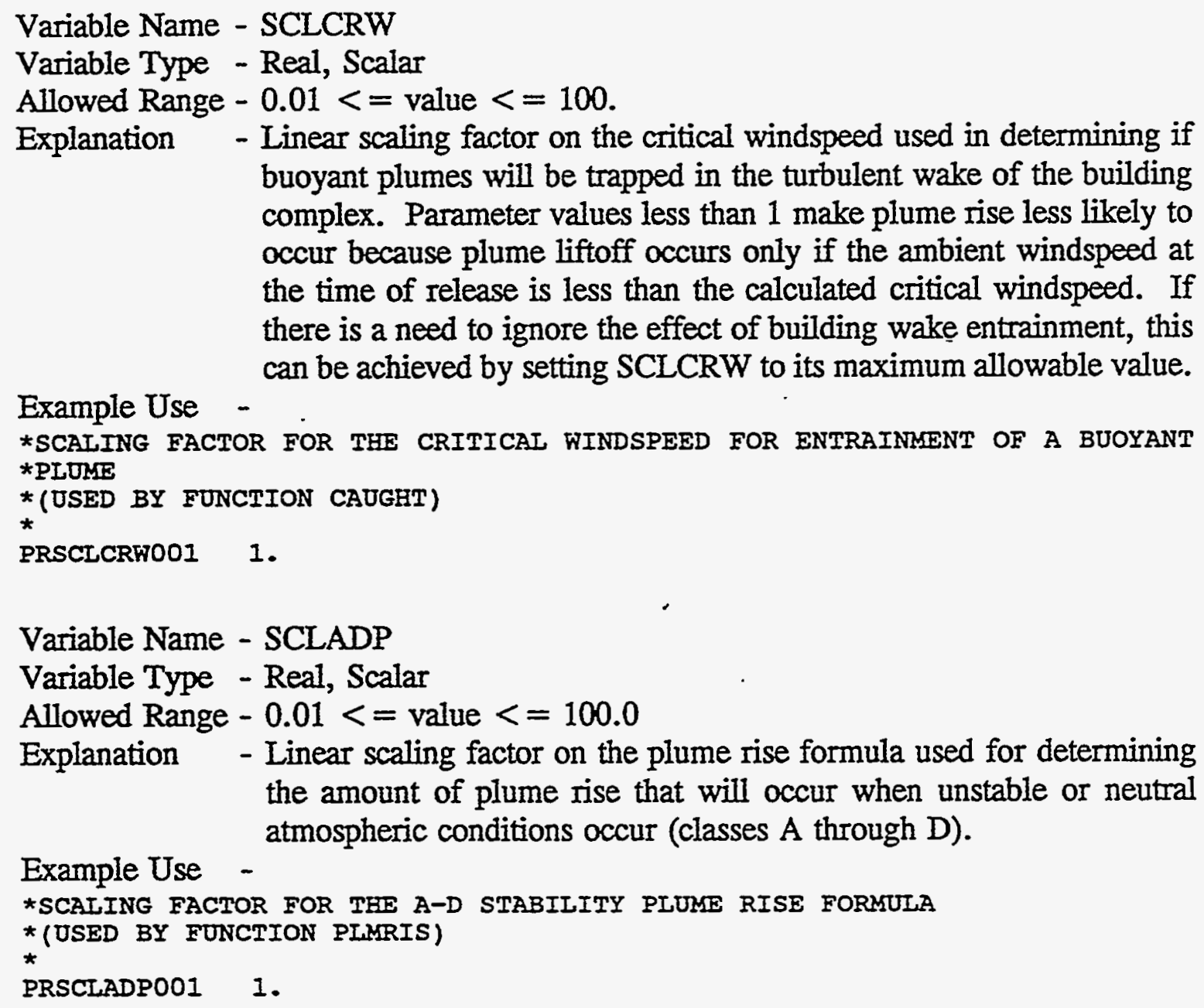
buoyant plumes will be trapped in the turbulent wake of the building complex. Parameter values less than 1 make plume rise less likely to occur because plume liftoff occurs only if the ambient windspeed at the time of release is less than the calculated critical windspeed. If there is a need to ignore the effect of building wake entrainment, this can be achieved by setting SCLCRW to its maximum allowable value.

Example Use -

*SCALING FACTOR FOR THE CRITICAL WINDSPEED FOR ENTRAINMENT OF A BUOYANT $\star$ PLUME

* (USED BY FUNCTION CAUGHT)

PRSCLCRFOOI 1 .

Variable Name - SCLADP

Variable Type - Real, Scalar

Allowed Range $-0.01<=$ value $<=100.0$

Explanation - Linear scaling factor on the plume rise formula used for determining the amount of plume rise that will occur when unstable or neutral

Example Use atmospheric conditions occur (classes $A$ through $D$ ).

*SCALING FACTOR FOR THE A-D STABIIITY PLUME RISE FORMULA

* (USED BY FUNCTION PIMRIS)

*

PRSCLADPOOI 1. 


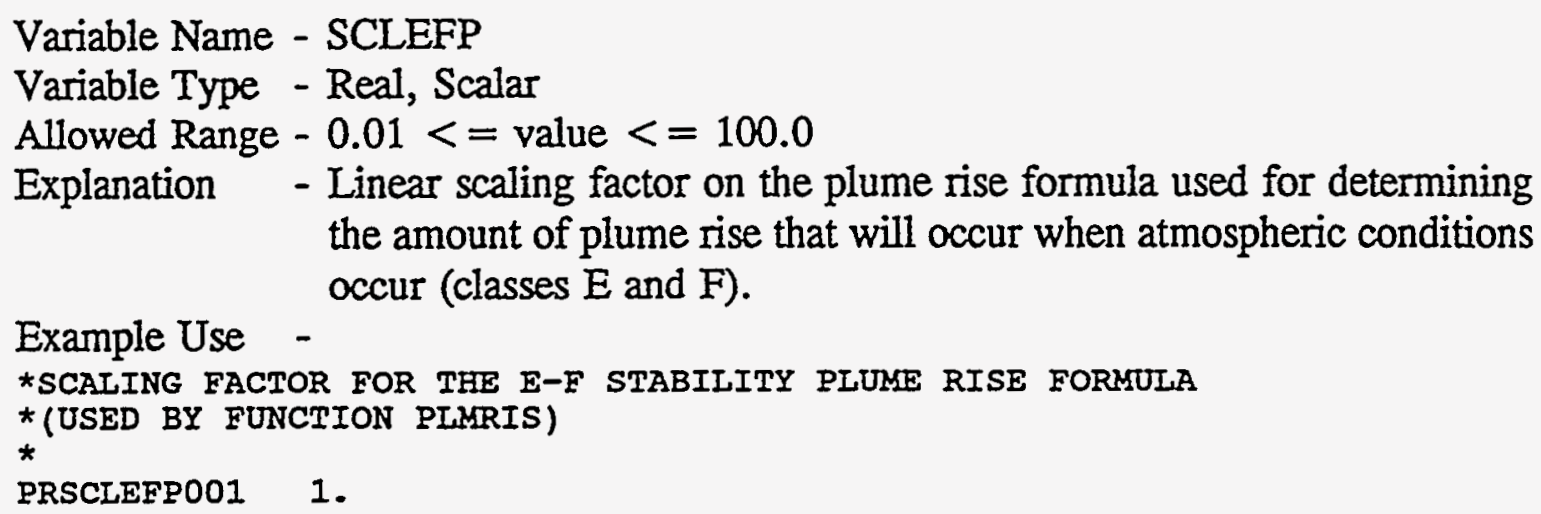

\section{B.10 Wake Effects (WE) Data}

The initial size of the plume is determined by the width and height of the building wake. Sigma-y is initialized to width/4.3, and sigma-z is initialized to height/2.15. The height of the building wake is also used to determine if the plume is entrained in the turbulent region surrounding the building. Consequently, in addition to determining the initial plume size, the wake height is utilized in the determination of whether buoyant plume rise will occur.

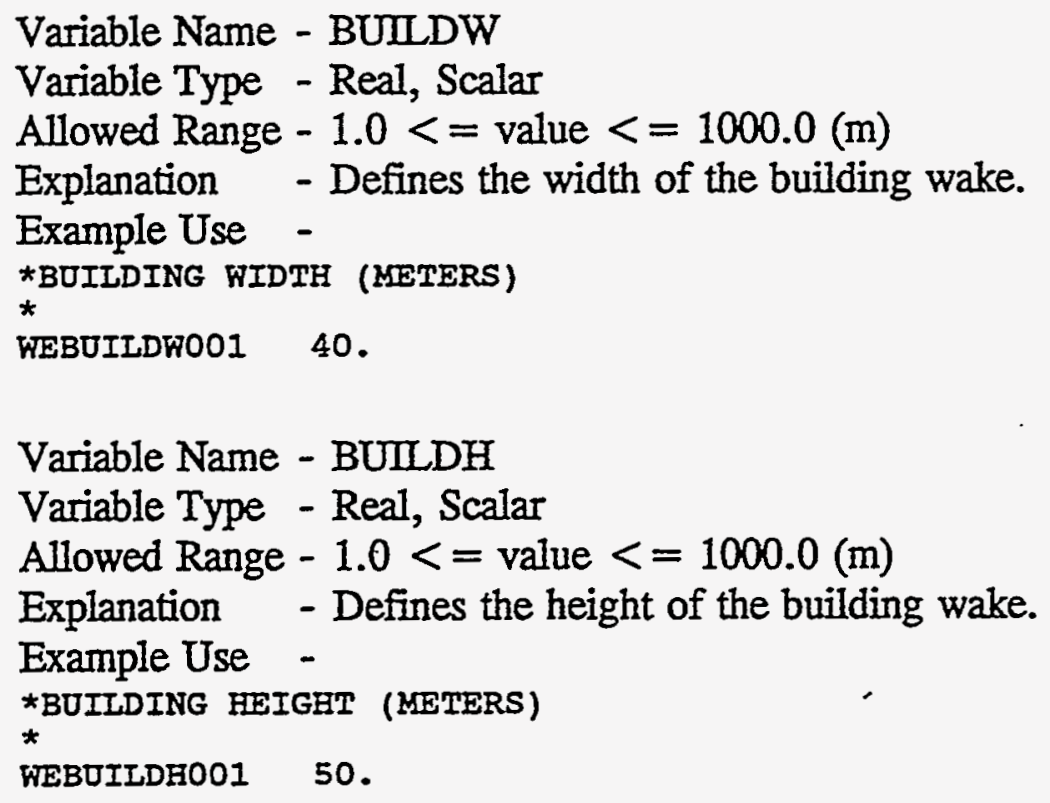

\section{B.11 Release Description (RD) Data}

ATMOS can handle multiple plume segments in order to treat releases with compositions that vary with time. The plume segments that comprise a release can be separated by a time gap, or can directly follow the preceding segment, but they are not allowed to overlap. The plume segments can be released at different heights, have different heat contents, and have different directions, but they must all share the same initial deposition velocity distribution. 
CHEM_MACCS incorporates the capability for calculating the consequences from up to 60 different source terms in a single run of the code. This is accomplished by appending "change cards" to the ATMOS User Input File. The first source term is defined in the main body of the ATMOS User Input File. Up to 59 additional source terms can be defined through change card sets positioned at the end of the file.

The delimiter used to separate the change card sets is a period (.) in column one. The end of the file is also signified by a period in column one. All of the CHEM_MACCS User Input Files must end with a period in column one. The sample ATMOS user input file listed in Appendix D of the MACCS User's Guide illustrates the use of change cards.

Change cards in the ATMOS user input file are used solely to modify the previously specified Release Description (RD) Data. If items from another data block appear in the change cards, they will be ignored. Each set of change cards must include a new value of ATNAM2, a text field describing the source term. Also, each set of change cards must specify a change in at least one of the numeric input variables described in this data block.

Variable Name - ATNAM2

Variable Type - Character, Scalar

Allowed Range $-1<=$ length $<=80$

Explanation - Identifies the name of the source term being studied. This name will be printed on all pages of the output listing. A unique name must be

Example Use specified for each source term.

* SPECIFIC DESCRIPTIVE TEXT DESCRIBING THIS PARTICULAR SOURCE TERM

RDATNAM2001 'CHEM_MACCS EXAMPLE SOURCE TERM MIX'

Variable Name - OALARM

Variable Type - Real, Scalar

Allowed Range $-0 .<=$ value $<=604800$. seconds (one week)

Explanation - Defines the time at which notification is given to offsite emergency response officials to initiate protective measures for the surrounding population. This time is a function of the accident sequence. It is measured from accident initiation.

Example Use -

* TIME AFTER aCCIDENT INITIATION WHEN THE ACCIDENT REACHES GENERAL

* EMERGENCY CONDITIONS (AS DEFINED IN NUREG-0654), OR WEEN PLANT PERSONNEL

* CAN REIIABLY PREDICT THAT GENERAI EMERGENCY CONDITIONS WIII BE ATTAINED

*

RDOALARMOOI 1300. (5)

Variable Name - NUMREL

Variable Type - Integer, Scalar

Allowed Range $-1<=$ value $<=4$ 
Explanation - Defines the number of plumes that will be released. If the multiple source term feature is being used, it is not possible to provide a larger value for NUMREL in the change cards than the value that was defined initially. That is, the values of NUMREL specified on change cards cannot be larger that the value of NUMREL specified for the base case.

Example Use -

* NUMBER OF PLUME SEGMENTS THAT ARE RELEASED

RDNUMRELOO1

2

Variable Name - MAXRIS

Variable Type - Integer, Scalar

Allowed Range $-1<=$ value $<=$ NUMREL

Explanation - Specifies which plume segment is to be considered risk dominant. The selection of the risk-dominant plume is usually based on its potential for causing early fatalities. Release of the risk-dominant plume always begins at the selected meteorological start time of the

Example Use weather sequence.

* SELECTION OF RISK DOMINANT PLUME

RDMAXRISOO1

1

Variable Name - REFTIM

Variable Type - Real, Array

Allowed Range -0 . $<=$ value $<=1$.

Explanation - Specifies the representative time point of each plume segment. ( $0 .=$ leading edge, $0.5=$ midpoint, $1 .=$ trailing edge). The characteristics of a plume are uniform along its length. This parameter allows the user to locate the contents of the plume in a "bucket" of material situated at some point within the plume's length. The dry deposition, and dispersion calculations are all performed as if the entire contents of the plume segment are located at this point. The user must supply NUMREL values of REFTIM, one for each plume segment. The choice of REFTIM has no impact on the wet deposition calculations since those are performed as if the entire

Example Use contents of the plume are uniformly distributed along its length.

* REPRESENTATIVE TIME POINT FOR DISPERSION

$\star$

RDREFTIMOOI $0.00 \quad 0.50 \quad$ (CORRESPONDING TO HEAD AND MIDPOINT WEATHER)

Variable Name - PLHEAT

Variable Type - Real, Array

Allowed Range -0 . $<=$ value $<=1$. E10 (W)

Explanation - Specifies the release rate of sensible heat in each plume segment. This quantity should be calculated as the amount of sensible heat in the 
Example Use -

plume segment divided by the duration of the plume segment. The value specified here is used to determine the amount of buoyant plume rise that will occur. The user must supply NUMREL values of PLHEAT, one for each plume segment.

* hEAT CONTENT OF THE RELEASE SEgMENTS (

* A VALUE SPECIFIED FOR EACH OF THE RELEASE SEgMENTS

RDPLHEATOO1 $3.7 \mathrm{E}+6 \quad 1.7 \mathrm{E}$

Variable Name - PLHITE

Variable Type - Real, Array

Allowed Range $-0 .<=$ value $<=1000$. (m)

Explanation - Specifies the height above ground level at which each plume segment is released. The user must supply NUMREL values of PLHITE, one

Example Use for each plume segment.

* HEIGHT OF THE PLUME SEGMENTS AT RELEASE (M)

* A VALUE SPECIFIED FOR EACH OF THE RELEASE SEGMENTS

*

RDPLEITEOOI $\quad 0 . \quad 0$.

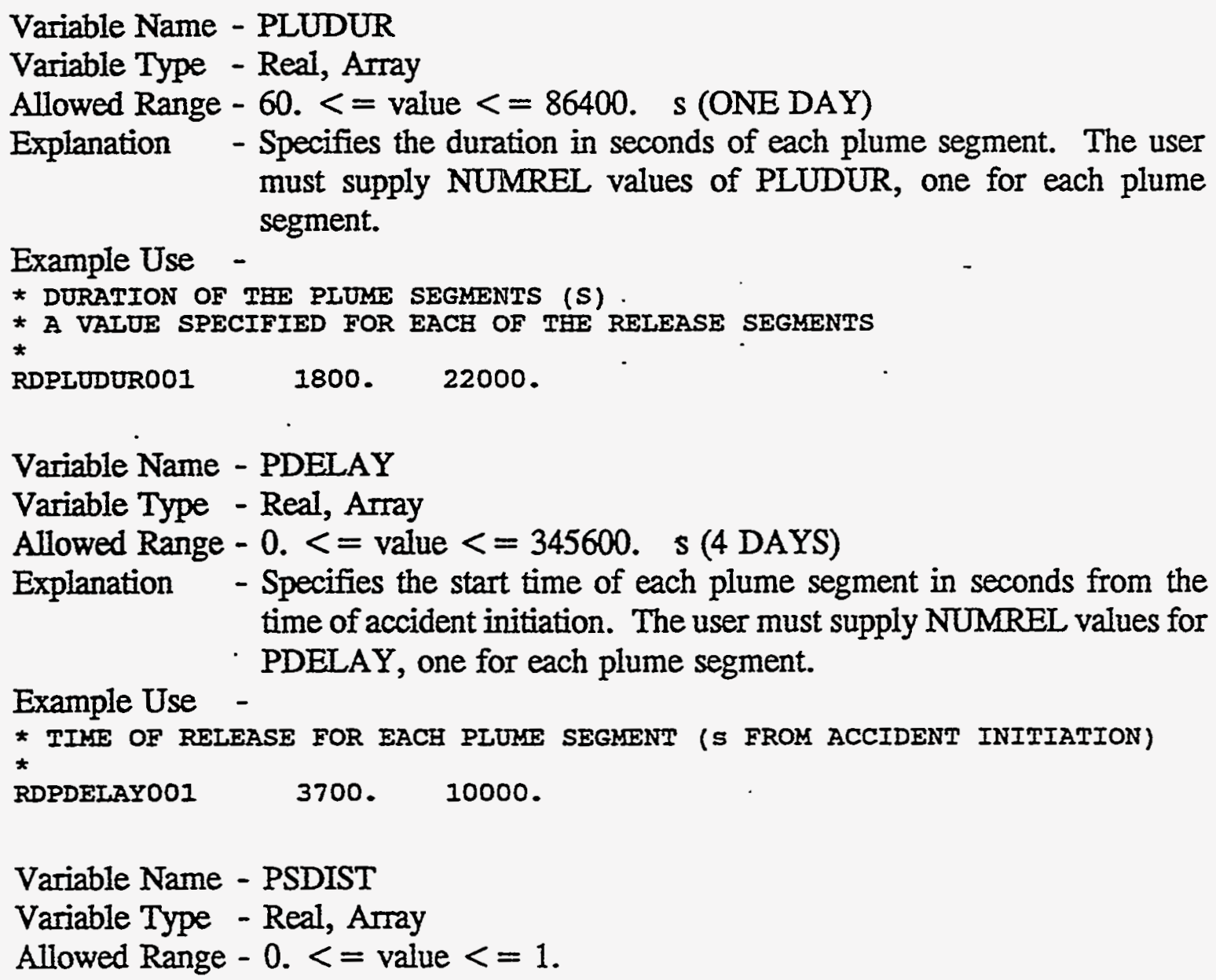
must supply NUMREL values of PLUDUR, one for each plume time of accident initiation. The user must supply NUMREL values for PDELAY, one for each plume segment.

Example Use -

* TIME OF RELEASE FOR EACH PLUME SEGMENT (S FROM ACCIDENT INITIATION)

RDPDELAYOO1 3700. 10000.

Variable Name - PSDIST

Variable Type - Real, Array

Allowed Range $-0 .<=$ value $<=1$. 
Explanation - Specifies the fraction of the released material allocated to each of the dry deposition velocities. All of the plume segments must use the same initial dry deposition velocity distribution. The user must use one input record to specify a dry deposition velocity distribution for each of the NUMISO chemical species. On each of these input records, the user must allocate a fraction of the released mass of each

Example Use chemical species to each deposition velocity.

* DEPOSITION VELOCITY DISTRIBUTION OF EACH CHEMICAL SPECIES
* THE FRACTIONS FOR EACH CHEMICAL SPECIES (ROW) MUST SUM TO ONE.

*

$\star$

*

RDPSDISTO01

RDPSDISTO02

RDPSDISTO03

RDPSDISTO04

RDPSDISTO05

RDPSDISTO06

RDPSDIST007

RDPSDIST008

$0.001 \mathrm{~m} / \mathrm{s} \quad 0.01 \mathrm{~m} / \mathrm{s}$

$0.0 \quad 1.0 \quad$ (GA-IIQ)

$0.0 \quad 1.0 \quad$ (GB-IIQ)

$0.0 \quad 1.0 \quad$ (VX-IIQ)

$0.0 \quad 1.0 \quad$ (HD-IIQ)

$1.0 \quad 0.0 \quad$ (GA-VAP)

$1.0 \quad 0.0 \quad$ (GB-VAP)

$1.0 \quad 0.0 \quad$ (VX-VAP)

$1.0 \quad 0.0 \quad$ (HD-VAP)

Variable Name - CORINV

Variable Type - Real, Array

Allowed Range $-0 .<=$ value $<=1$. E35 $(\mathrm{kg})$

Explanation - Specifies the inventory of each chemical species that is available for release. All of the chemical species that are defined via the NUCNAM array (Section B.4) must be listed here. The chemical

Example Use species may be listed in any order.

* INVENTORY OF

$$
\begin{array}{ll}
* \\
\star \\
* \\
* \\
*
\end{array}
$$

RDCORINYOO1

RDCORINVOO2

RDCORINVOO3

RDCORINVOO4

RDCORINVO05

RDCORINVO06

RDCORINVOO7

RDCORINVO08

\section{CHEMICAL}

SPECIES

Variable Name - CORSCA

GA-IIQ

VX-IIQ

HD-IIQ

GA-VAP

GB-VAP

VX-VAP

HD-VAP
SPECIES S
QUANTITY
RELEASED

$(\mathrm{kg})$

Variable Type - Real, Scalar

Allowed Range - 1.E-35 $<=$ value $<=1 . E+35$

Explanation

- This is a linear scaling factor that can be used to adjust the inventory of all of the chemical species included in the model. CORSCA can also be used to convert the inventory from one set of units to another, thereby avoiding the tedium of manually converting a set of numbers. For example, to convert from pounds to kilograms, use a value of $0.4535924 \mathrm{~kg} / \mathrm{lb}$. 


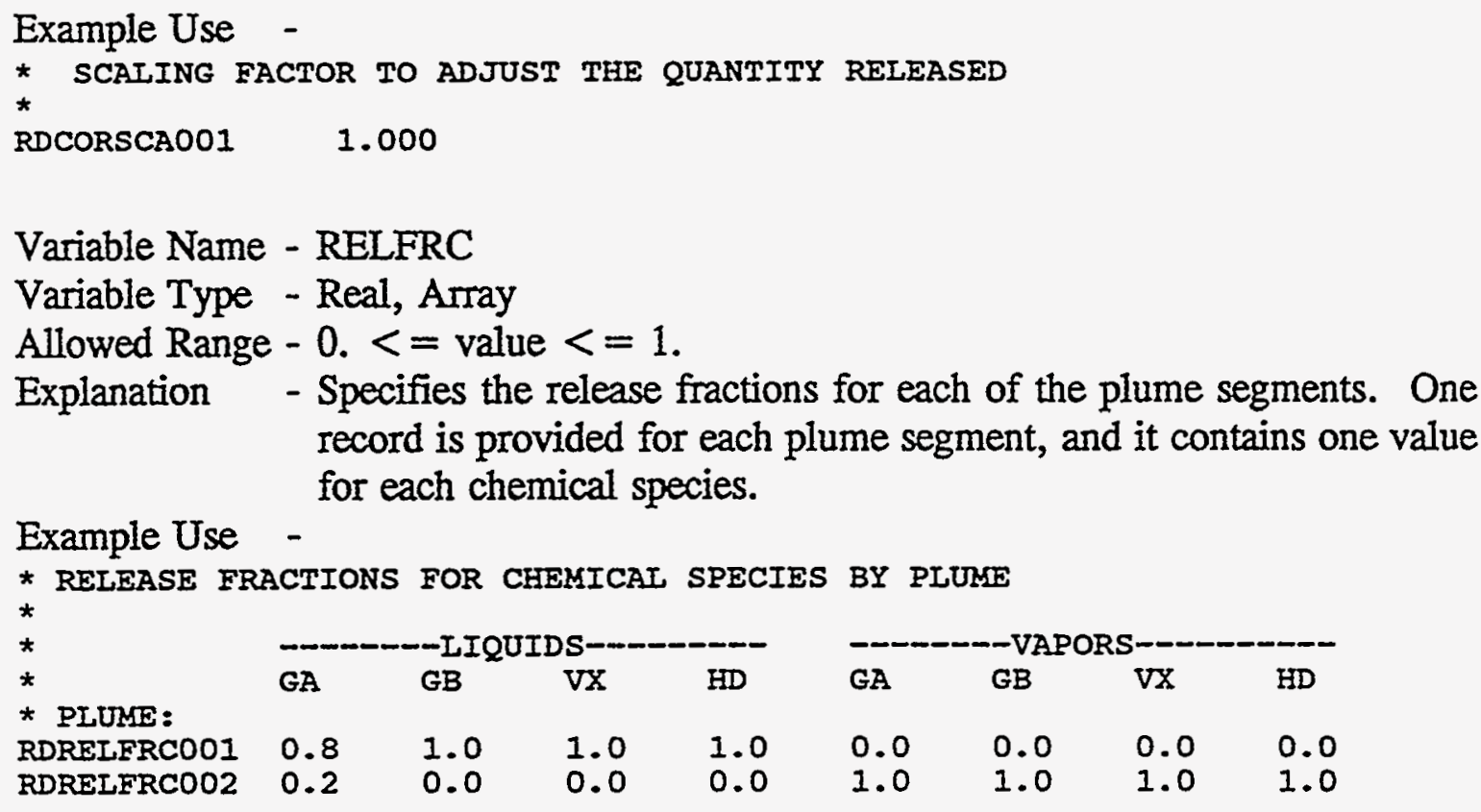
record is provided for each plume segment, and it contains one value for each chemical species.

Example Use -

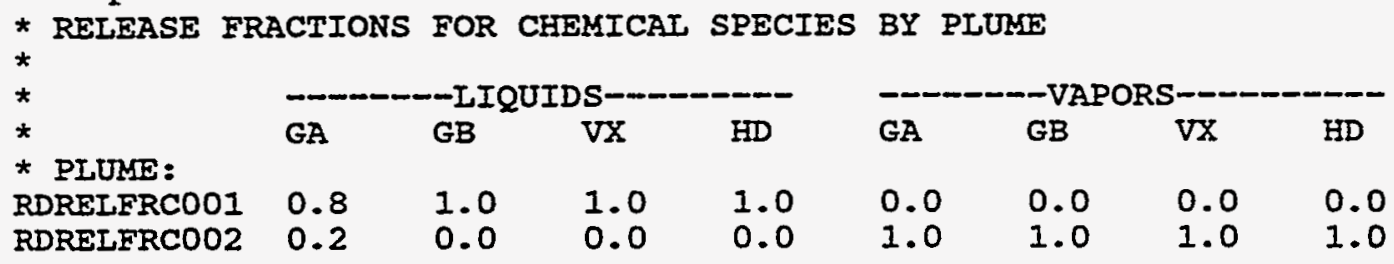

\section{B.12 Output Control (OC) Data}

The user has the option of looking at tables of dispersion data for all of the trials that are performed. This information includes air and ground concentrations, sigma-y and sigma-z values, and the arrival and departure times for each plume segment at each spatial interval. Then data are written to the standard output file (unit 6).

Variable Name - ENDAT1

Variable Type - Logical, Scalar

Allowed Value - .TRUE. or .FAISE.

Explanation - Control flag that allows the user to execute only the ATMOS module. A value of .TRUE. tells the code that EARLY will not be run. When this is done, the User Input Files for EARLY and a Site Data File need not be supplied.

Example Use -

*FLAG TO INDICATE THAT THIS IS THE LAST PROGRAM IN THE SERIES TO BE RUN *

OCENDAT1001 -FAISE. (SET THIS VALUE TÓ .TRUE. TO SKIP EARLY)

Variable Name - IDEBUG

Variable Type - Integer, Scalar

Allowed Range $-0<=$ value $<=8$

Explanation - Specifies the quantity of debug output to be printed. For normal runs, IDEBUG should be set to zero (i.e., no debug output is printed). If IDEBUG is set to one or two, a print of the atmospheric transport results described below will be generated for each weather trial and each plume segment. If IDEBUG is set to a value of three or more the hourly meteorological data that were used for each weather trial will also be printed. 
The debug output for atmospheric transport prints the results described below.

NUCNAM - name of the chemical species for which results are being presented,

DISTANCE - distance to the center of the spatial interval (m),

GL AIRCON - centerline ground-level integrated air concentration from this plume segment averaged over the spatial interval's length $\left(\mathrm{kg}-\mathrm{s} / \mathrm{m}^{3}\right)$,

GRNCON - centerline ground concentration after passage of this plume averaged over the spatial interval's length $\left(\mathrm{kg} / \mathrm{m}^{2}\right)$,

GL X/Q - centerline ground level chi over $Q(\alpha / Q)$, ratio of air concentration, chi, to source strength $Q$, in SI units, averaged over the interval's length,

WETREM - fraction of material remaining in the plume segment after wet deposition over the spatial interval's length,

DRYREM - fraction of material remaining in the plume segment after dry deposition over the spatial interval's length,

REMINV - adjusted source strength of the plume upon entering each spatial interval after adjustment for losses in the previous intervals due to wet and dry deposition $(\mathrm{kg})$,

PLSIGY - horizontal dispersion parameter sigma-y averaged over the spatial interval's length (m),

PLSIGZ - horizontal dispersion parameter sigma- $z$ averaged over the spatial interval's length (m),

WEATHER - indices to the first and last hours of the weather sequence used for determining atmospheric conditions during transport across each spatial interval,

HTFCTR - Tatio of the centerline ground-level air concentration $(z=0)$ to the plume centerline air concentration $(\mathrm{z}=\mathrm{H})$,

AVGHTT - average height $(\mathrm{H})$ of the plume as it traversed the spatial interval (m),

TIMCEN - time after accident initiation at which the leading edge of the plume arrived at the center of the spatial interval (s),

TIMOVH - duration for which the plume was overhead at the centerpoint of the spatial interval (s).

Example Use -

OCIDEBUGO01 I (REQUEST A TRACE OF ATMOSPHERIC DISPERSION)

Variable Name - NUCOUT

Variable Type - Character, Scalar

Allowed Range $-3<=$ length $<=8$

Explanation - Specifies which chemical species will appear on the dispersion listing if one is produced. The dispersion listing is only produced if DEEUUG is greater than zero. The specified chemical species name must appear on the previously defined list of chemical species, NUCNAM, defined in Section 6.4. This item is only required if DEBUG is greater than zero.

Example Use

*NAME OF THE CHEMICAI SPECIES TO BE IISTED ON THE DISPERSION LISTINGS OCNUCOUTOOI GA-VAP 


\section{B.13 Meteorological Sampling (M1) Specification}

There are five options available to the user for specifying the weather data that will be used by ATMOS. The code can be used to run either a single weather sequence or multiple weather sequences.

If a single weather sequence is desired, there are three ways to specify the weather. The user can either (1) specify data for $120 \mathrm{hr}$ of weather on the ATMOS User Input File, (2) specify a starting day and hour in the weather data file for the weather sequence, or (3) specify constant weather conditions. For the specified starting day and hour option, the program will obtain $120 \mathrm{hr}$ of weather data from the weather file beginning at the specified date and time. A file of hourly weather data covering a period of 1 year $(8,760 \mathrm{hr})$ is required if the fixed start time or either of the weather sampling options are to be used. The format of this file is described in Appendix D.

The two methods of weather sampling are (1) a modified version of the weather bin sampling method used by $\mathrm{CRAC} 2^{4}$ and (2) a stratified purely random sampling approach.

The weather bin sampling method sorts weather sequences into categories and assigns a probability to each according to their initial conditions (windspeed and stability class) and the occurrence of rain (intensity and distance). Because the rain bins depend on rain intensity as well as the downwind distance at which rain occurs, the user is required to supply parameters defining the rain weather bins as part of the ATMOS User Input File. The definitions of the other weather bins, those defined by initial stability class and windspeed, are hard wired in the code. The definitions of those initial condition weather bins are not the same as used in CRAC2. In addition to changing the breakpoints used to categorize windspeed, the windspeed slowdown bins of CRAC2 have been removed. A description of the MACCS weather sampling algorithm can be found in the MACCS Model Description. ${ }^{2}$

The stratified random sampling method allows the user to sample weather from each day of the year after division of each day into one, two, three, or four equal time periods. Each weather sequence selected is considered to have the same probability of occurrence, that is,

$$
P=\frac{1}{\text { total selected samples }}
$$

Because of the flexibility that ATMOS affords in the specification of the geometric grid, it is necessary to guard against the possibility of running out of weather data. It is possible that 120 hr of weather may not suffice to carry all the plume segments out to the last spatial interval. Also, the user may wish to cause the occurrence of rain in the outermost spatial intervals in order to prevent chemical species from escaping consideration. For these reasons, the user must specify a set of boundary weather conditions. 
Depending on the options selected by the user, different data items will be required by ATMOS.

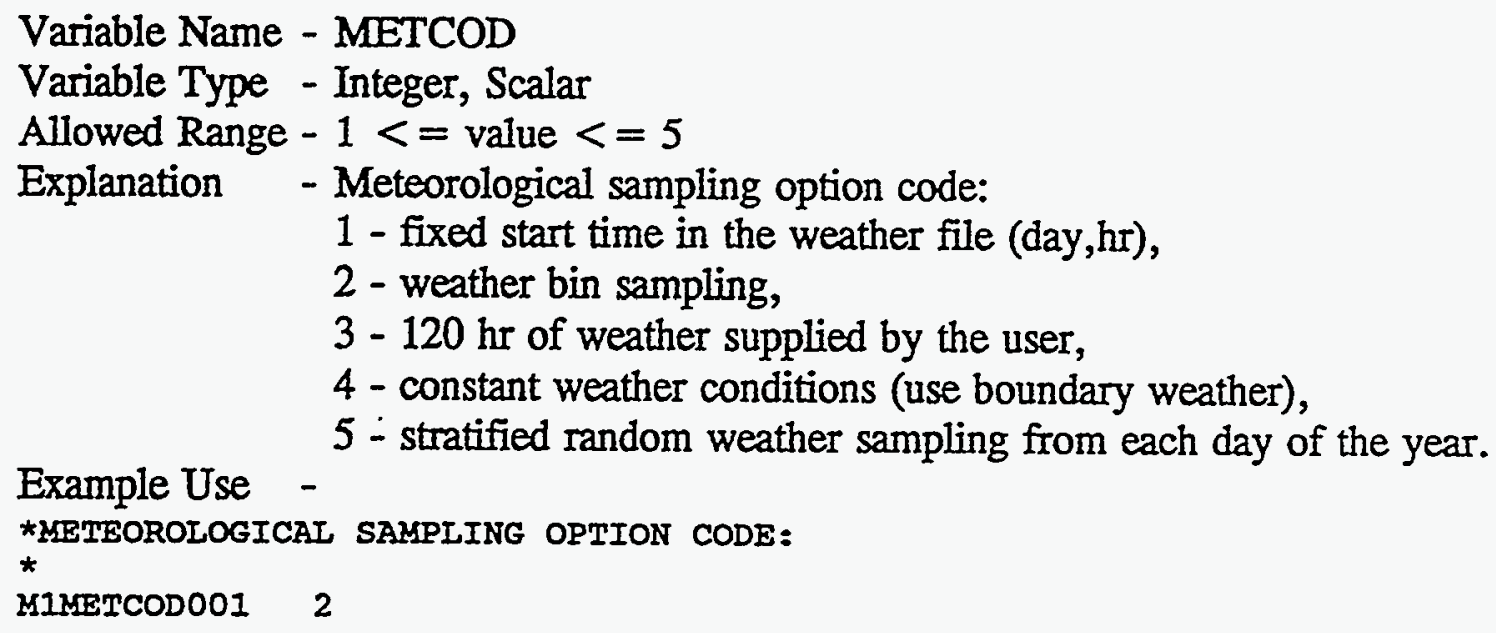

\section{B.14 Boundary Weather (M2) Data}

Boundary Weather Data are required for all possible values of METCOD. This data block specifies the weather conditions that will be used if $120 \mathrm{hr}$ of recorded weather data do not transport the last plume through the limiting spatial interval for measured weather, LIMSPA. The boundary weather data are also used for predicting the behavior of the plume at all spatial intervals beyond LIMSPA.

For the case of constant weather, METCOD-4, the boundary weather data in this section determine the constant weather conditions that will be used. The boundary weather is used throughout the atmospheric calculations and the value of LIMSPA is ignored by the program in this case.

Variable Name - LIMSPA

Variable Type - Integer, Scalar

Allowed Range - $0<=$ value $<=$ NUMRAD

Explanation - This is the limiting spatial interval for use of recorded weather data. All spatial intervals beyond this spatial interval will use the boundary weather conditions specified below. If a value of zero is specified, then the boundary weather conditions will be used right from the start.

Example Use If METCOD-4, the value of LIMSPA is ignored.

*LAST SPATIAI INTERVAI FOR MEASURED WEATHER

$\star$

K2LIMSPAOO1 25

Variable Name - BNDMXXH

Variable Type - Real, Scalar

Allowed Range $-1 . \mathrm{E} 2<=$ value $<=1$.EA (m) 
Explanation - This is the mixing layer height that will be used for the boundary weather conditions.

Example Use -

*BOUNDARY WEATHER MIXING IAYER HEIGHT

M2BNDMXBO01 1000. (METERS)

Variable Name - IBDSTB

Variable Type - Integer, Scalar

Allowed Range $-1<=$ value $<=6$

Explanation - This is the stability class that will be used for the boundary weather conditions. The integers 1 through 6 represent Pasquill-Gifford

Example Use stability classes A through $F$, respectively.

*BOUNDARY WEATHER STABILITY CLASS INDEX

*

M2IBDSTB001 1 (A-STABILITY)

Variable Name - BNDRAN

Variable Type - Real, Scalar

Allowed Range $-0 .<=$ value $<=99$. $(\mathrm{mm} / \mathrm{hr})$

Explanation - This is the rain rate that will be used for the boundary weather conditions.

Example Use -

*BOUNDARY WEATHER RAIN RATE

*

M2BNDRANO01 O. (O MM/HR = NO RAIN)

Variable Name - BNDWND

Variable Type - Real, Scalar

Allowed Range $-0.5<=$ value $<=30$. $(\mathrm{m} / \mathrm{s})$

Explanation - This is the wind speed that will be used for the boundary weather conditions.

Example.Use -

*BOUNDARY WEATHER WIND SPEED

*

M2BNDWND001 $0.5 \quad(\mathrm{M} / \mathrm{S})$

\section{B.15 Fixed Start Time (M3) Data}

The data in this section must be supplied for all values of METCOD except METCOD-2 (meteorological bin sampling), and METCOD-5 (stratified random sampling).

If the user has chosen METCOD =1, the values of ISTRDY and ISTRHR specify the starting day and hour in the weather file of the single weather trial that will be performed. 


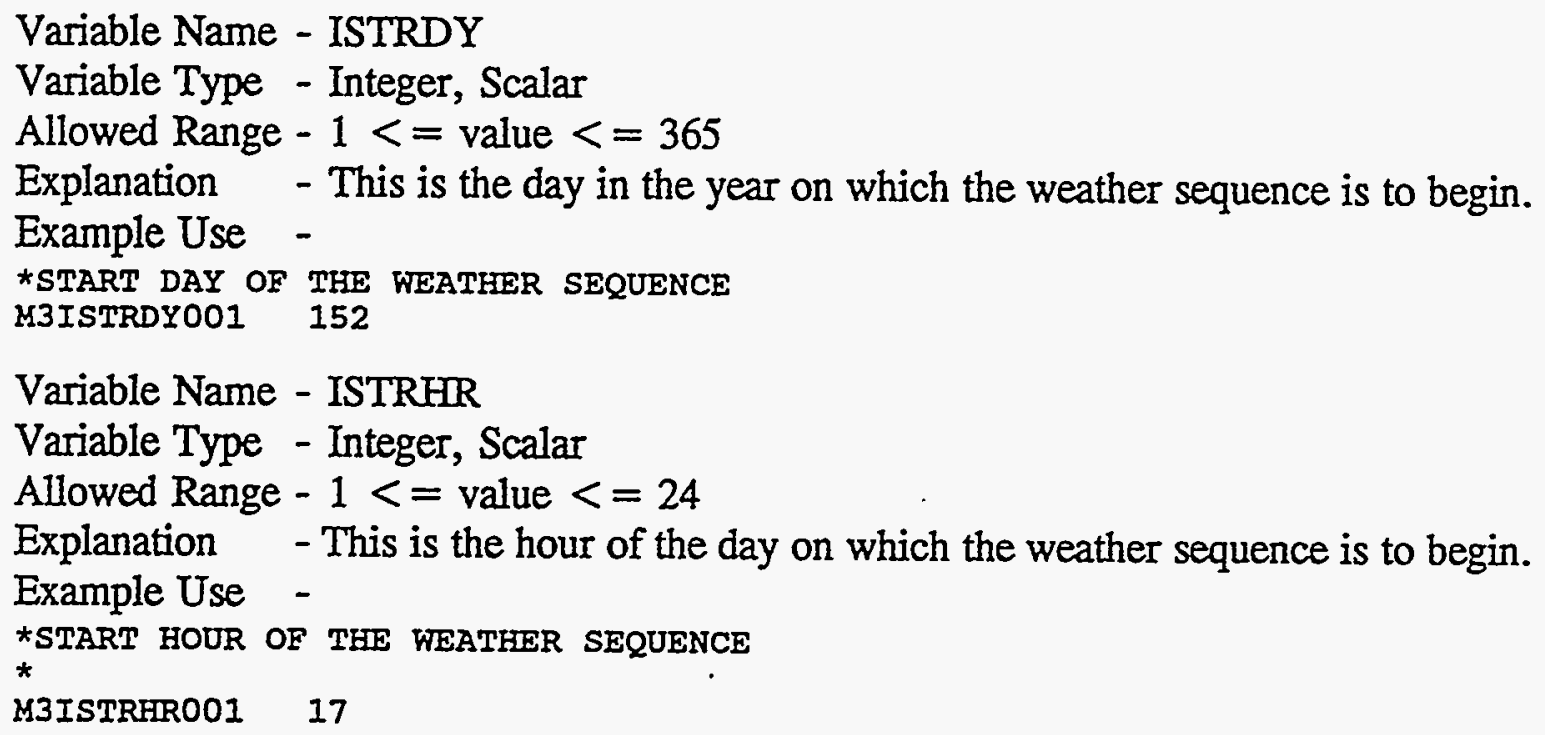

\section{B.16 Meteorological Bin Sampling (M4) Data}

The data in this section must be supplied if the user chooses METCOD $=2$. This sampling method requires that the meteorological data be sorted into a set of weather bins. The bins are defined to represent rain conditions in different distance intervals downwind from the accident site together with sixteen bins for the initial conditions (stability class and wind speed).

Definition of the rain intensities and distance intervals that define the rain bins is the responsibility of the user. The user must specify either two or three rain intensities which are used as breakpoints in the categorization of rain rate (NRINTN). A rain intensity of zero is not allowed. For example, if the user specifies two rain intensity breakpoints of $1 \mathrm{~mm} / \mathrm{hr}$ and $4 \mathrm{~mm} / \mathrm{hr}$, the following three rain intensity bins, where $\mathrm{x}$ is the rain intensity, will result:

1. $0 \mathrm{~mm} / \mathrm{hr} \cdot<\mathrm{x}<1 \mathrm{~mm} / \mathrm{hr}$,

2. $1 \mathrm{~mm} / \mathrm{hr}<\mathrm{x}<4 \mathrm{~mm} / \mathrm{hr}$,

3. $4 \mathrm{~mm} / \mathrm{hr}<\mathrm{x}$.

The concept of rain distance intervals used in MACCS is similar to that used by CRAC2 but MACCS requires that the user specify the rain distances to be used in the weather categorization. ${ }^{1,4}$ Suppose the user specifies $2,4,8$, and $16 \mathrm{~km}$ as four distance intervals (NRNINT). Then these values define the following four rain distance intervals:

1. $0 \mathrm{~km}<=$ distance of first rain occurrence $<=2 \mathrm{~km}$,

2. $2 \mathrm{~km}<=$ distance of first rain occurrence $<=4 \mathrm{~km}$,

3. $4 \mathrm{~km}<=$ distance of first rain occurrence $<=8 \mathrm{~km}$,

4. $8 \mathrm{~km}<=$ distance of first rain occurrence $<=16 \mathrm{~km}$.

The total number of weather bins, $\mathrm{N}$, is determined by the expression: 


$$
N=\text { NRNINT } *(\text { NRINTN }+1)+16
$$

where NRNINT is the number of distance intervals and NRINTN is the number of rain intensity breakpoints. Thus, the total number of defined bins can range from twentyeight to forty depending on the values supplied by the user.

The sixteen initial condition weather bins are hard wired into the code as follows:

$\begin{array}{ccl}\text { METBIN } & \text { STABILITY } & \text { WINDSPEED (u) } \\ 1 & \text { A/B } & 0 \mathrm{~m} / \mathrm{s}<\mathrm{u}<=3 \mathrm{~m} / \mathrm{s} \\ 2 & \text { A/B } & 3 \mathrm{~m} / \mathrm{s}<\mathrm{u} \\ 3 & \text { C/D } & 0 \mathrm{~m} / \mathrm{s}<\mathrm{u}<=1 \mathrm{~m} / \mathrm{s} \\ 4 & \text { C/D } & 1 \mathrm{~m} / \mathrm{s}<\mathrm{u}<=2 \mathrm{~m} / \mathrm{s} \\ 5 & \text { C/D } & 2 \mathrm{~m} / \mathrm{s}<\mathrm{u}<=3 \mathrm{~m} / \mathrm{s} \\ 6 & \text { C/D } & 3 \mathrm{~m} / \mathrm{s}<\mathrm{u}<=5 \mathrm{~m} / \mathrm{s} \\ 7 & \text { C/D } & 5 \mathrm{~m} / \mathrm{s}<\mathrm{u}<=7 \mathrm{~m} / \mathrm{s} \\ 8 & \text { C/D } & 7 \mathrm{~m} / \mathrm{s}<\mathrm{u} \\ 9 & \text { E } & 0 \mathrm{~m} / \mathrm{s}<\mathrm{u}<=1 \mathrm{~m} / \mathrm{s} \\ 10 & \text { E } & 1 \mathrm{~m} / \mathrm{s}<\mathrm{u}<=2 \mathrm{~m} / \mathrm{s} \\ 11 & \text { E } & 2 \mathrm{~m} / \mathrm{s}<\mathrm{u}<=3 \mathrm{~m} / \mathrm{s} \\ 12 & \text { E } & 3 \mathrm{~m} / \mathrm{s}<\mathrm{u} \\ 13 & \text { F } & 0 \mathrm{~m} / \mathrm{s}<\mathrm{u}<=1 \mathrm{~m} / \mathrm{s} \\ 14 & \text { F } & 1 \mathrm{~m} / \mathrm{s}<\mathrm{u}<=2 \mathrm{~m} / \mathrm{s} \\ 15 & \text { F } & 2 \mathrm{~m} / \mathrm{s}<\mathrm{u}<=3 \mathrm{~m} / \mathrm{s} \\ 16 & \text { F } & 3 \mathrm{~m} / \mathrm{s}<\mathrm{u}\end{array}$

Note that the definition of the initial condition weather categories is not the same as that used by CRAC2. Also, MACCS does not consider windspeed slowdowns in the weather categorization.

The user controls how many weather sequences are chosen from each weather bin by the choice of a value for NSMPLS, defined later in this section. This can be done in two different ways: either (1) request that the same number of weather sequences are to be chosen from each bin and specify the number of sequences, NSMPLS, to be selected $(1<=$ NSMPLS $<=10)$, or else (2) specify a nonuniform sampling from the categories (NSMPLS $=0)$ as defined below.

Variable Name - NRNINT

Variable Type - Integer, Scalar

Allowed Range $-4<=$ value $<=6$

Explanation - Defines the number of rain distance intervals used in the weather categorization. 
Example Use -

* NURBER OF RAIN DISTANCE INTERVALS FOR BINNING

MANRNINTOO1 5

Variable Name - RNDSTS

Variable Type - Real, Array

Allowed Range $-0.001<=$ value $<=99.9$ (kilometers)

Explanation - Defines the rain distance interval endpoints to be used for the weather categorization. These distance values must lie within $10 \%$ of the spatial interval endpoint distances (variable SPAEND in Section 6.3), i.e., $0.9 * \operatorname{SPAEND}(\mathrm{i})<=$ RNDSTS $(\mathrm{j})<=1.1 * \operatorname{SPAEND}(\mathrm{i})$, for each $j$ and some value of $i$. The user must supply NRNINT unique values

Example Use in ascending order.

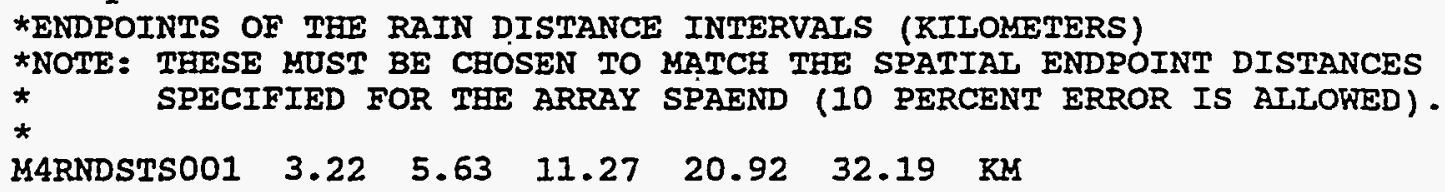

Variable Name - NRINTN

Variable Type - Integer, Scalar

Allowed Range $-2<=$ value $<=3$

Explanation - Defines the number of rain intensity breakpoints to be used for the weather categorization.

Example Use -

* NUMBER OF RAIN INTENSITY BREARPOINTS

*

MANRINTNOO1 3

Variable Name - RNRATE

Variable Type - Real, Array

Allowed Range $-0.001<=$ value $<=100.0(\mathrm{~mm} / \mathrm{hr})$

Explanation - Defines the rain intensity breakpoints. The user must supply NRINTN different values in ascending order.

Example Use -

*RAIN INTENSITY BREARPOINTS FOR WEATHER BINNING (MILIIMETERS PER HOUR)

$\star$

MARNRATEOO1 2. 4.6 . 6

Variable Name - NSMPLS

Variable Type - Integer, Scalar

Allowed Range $-0<=$ value $<=10$

Explanation - Defines the number of weather sequences to be chosen from each of the weather category bins. If the user supplies a value of zero, the following data records allow the user to specify how many samples are to be chosen from each bin. 
Example Use -

*NUMBER OF SAMPLES PER BIN

*

M4NSMPLSOOI 4 (THIS NUMBER SHOULD BE SET TO 4 FOR RISK ASSESSMENT)

Variable Name - IRSEED

Variable Type - Integer, Scalar

Allowed Range $-0<=$ value $<=255$

Explanation - Defines the initial seed of the random number generator. Changes to this value will cause different weather sequences to be selected. The random number generator of MACCS is included in the FORTRAN source code and therefore runs made on different types of computers

Example Use should select identical sets of weather sequences.

*INITIAI SEED FOR RANDOM NUMBER GENERATOR

MAIRSEEDOO1 79

Note: the following cards in this section are only needed if NSMPLS $=0$.

Variable Name - NSBINS

Variable Type - Integer, Scalar

Allowed Range $-1<=$ value $<=N$, total number of weather bins

Explanation - Defines the list of weather bins from which weather sequences are to be selected. The user must supply NSBINS values in column one of the data block. In order to find the index number to a rain bin, please refer to the page of the output listing " with the title "METEOROLOGICAL BIN SUMMARY."

Variable Name - INWGHT

Variable Type - Integer, Array

Allowed Range $-1<=$ value $<=8760$

Explanation - Defines the number of weather sequences the user would like to be selected from the specified weather bin. If the requested number of sequences cannot be found, the code will select all of the sequences in the specified bin. The user must supply NSBINS values in column two of the data block.

Example Use -

\begin{tabular}{|c|c|c|}
\hline BIN & N NUMBER & SAMPIE SIZE \\
\hline$\star \quad I$ & INDXBN & INFGET \\
\hline M4SMPIDFOOI & 13 & 8 \\
\hline M4SMPIDFOO2 & 4 & 16 \\
\hline K4SMPIDFOO 3 & 5 & 12 \\
\hline K4SMPIDDFOO4 & 6 & 4 \\
\hline M4SHPIDFOO5 & 7 & 4 \\
\hline M4SMPIDFOO6 & 8 & 4 \\
\hline
\end{tabular}




\section{B.17 User-Supplied Weather Sequence (M5) Data}

The data in this section must be supplied if the user chooses METCOD=3. There must be one data card for each hour of weather in the sequence. The five arrays in this section are supplied in a block of data as columns.

Variable Name - HRMXHT

Variable Type - Real, Array

Allowed Range - 1.E2 < = length $<=1$.E4 (m)

Explanation - These are the mixing layer heights in meters that will be used for the single trial. The user must supply 120 values of ISTAB in column one of the data block.

Note: The atmospheric dispersion model currently being used cannot accommodate a mixing layer height that varies with time during a weather sequence. The single value of mixing height which will be used in the atmospheric model is the largest value in the following set of values: the 120 values supplied here, and the boundary weather mixing layer height, BNDMXH.

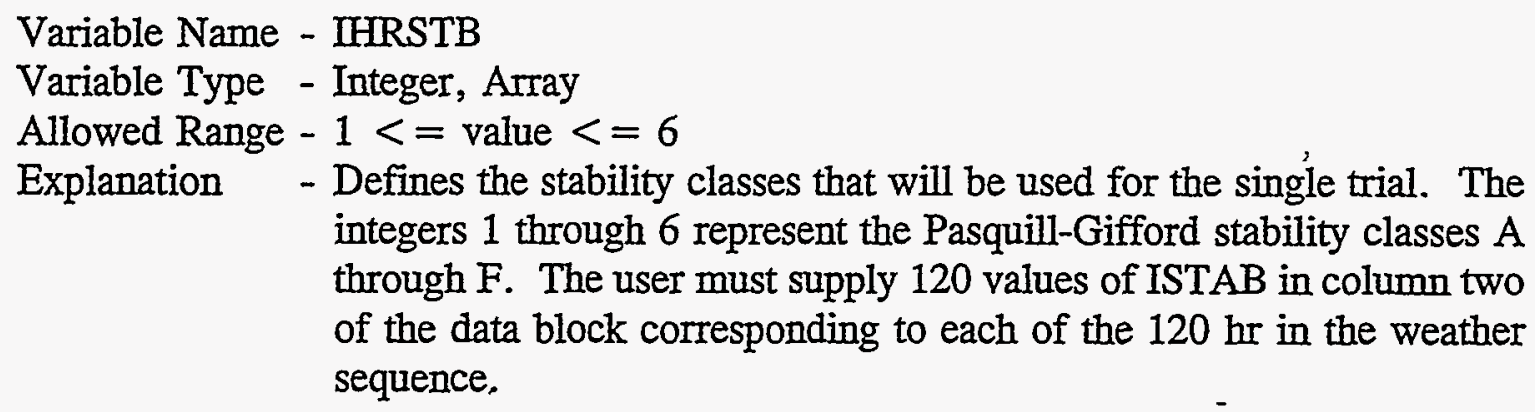
integers 1 through 6 represent the Pasquill-Gifford stability classes A through $\mathrm{F}$. The user must supply 120 values of ISTAB in column two of the data block corresponding to each of the $120 \mathrm{hr}$ in the weather sequence,

Variable Name - HRRAIN

Variable Type - Real, Array

Allowed Range $-0.0<=$ value $<=99.0(\mathrm{~mm} / \mathrm{hr})$

Explanation - Defines the rain rates that will be used for the single trial. They are given in units of millimeters per hour. The user must supply 120 values of RNMM in column three of the data block.

Variable Name - HRWNDV

Variable Type - Real, Array

Allowed Range $-0.5<=$ value $<=30.0(\mathrm{~m} / \mathrm{s})$

Explanation - Defines the wind speed that will be used for the single trial. They are given in units of meters per second. The user must supply 120 values of WINDSP in column four of the data block.

Variable Name - IHRDIR

Variable Type - Integer, Array 
Allowed Range $-1<=$ value $<=16$

Explanation - Defines the wind direction that will be used in the single trial. They are given as integers corresponding to the wind directions north through north-northwest. The user must supply 120 values of IHRDIR in column five of the data block.

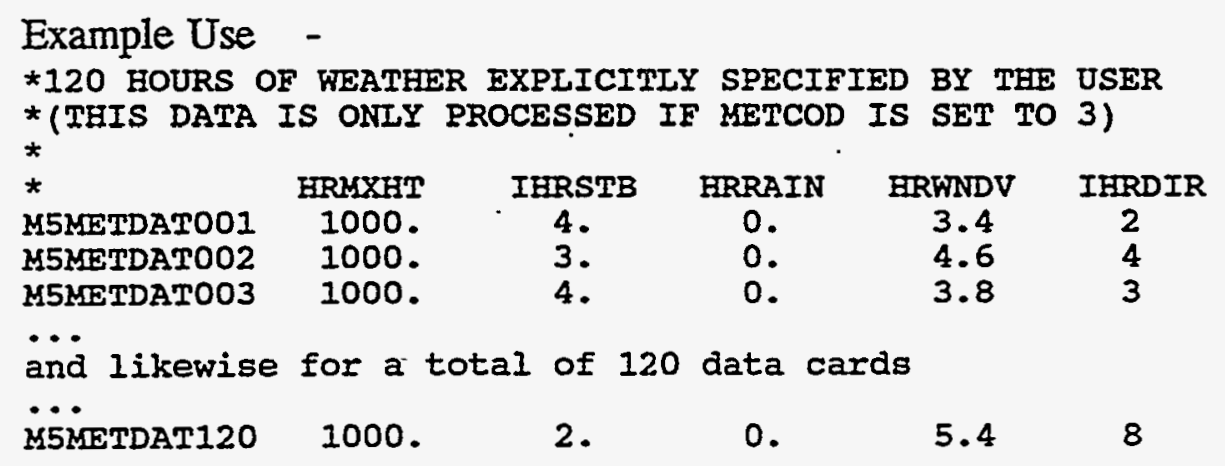

\section{References}

1. D. I. Chanin, J. L. Sprung, L. T. Ritchie, and H-N Jow, MELCOR Accident Consequence Code System (MACCS) User's Guide, NUREG/CR-4691, SAND861562, Vol.1, Sandia National Laboratories, Albuquerque, NM, February 1990.

2. H-N Jow, J. L. Sprung, J. A. Rollstin, L. T. Ritchie, and D. I. Chanin, MELCOR Accident Consequence Code System (MACCS), Model Description, NUREG/CR-4691, SAND86-1562, Vol. 2, Sandia National Laboratories, Albuquerque, NM, February 1990.

3. J.A. Rollstin, D.I. Chanin, and H-N Jow, MELCOR Accident Consequence Code System (MACCS), Programmer's Reference Manual, Sandia National Laboratories, Albuquerque, NM, NUREG/CR-4691, vol. 3, SAND86-1562, February 1990.

4. L. T. Ritchie, et al., CRAC2 Model Description, NUREG/CR-2552, SAND82-0342, Sandia National Laboratóries, Albuquerque, NM, 1984. 


\title{
APPENDIX C
}

\author{
Early Input File
}

\section{C.1 Introduction}

The EARLY module models the time period immediately following an accident. This period is commonly referred to as the emergency phase. It may extend up to 1 week after the arrival of the first plume at any downwind spatial interval. The user may specify scenarios that make use of evacuation, sheltering, and dose-dependent relocation. Results can be calculated for combinations of scenarios weighted either by time fractions (frequencies of occurrence) or population fractions (fraction of the population engaging in the specified behavior).

The EARLY module has the capability for combining results from up to three different emergency response scenarios. This is accomplished by appending "change cards" to the EARLY User Input File. The first emergency response scenario is defined in the main body of the EARLY User Input File. Up to two additional emergency response scenarios can be defined through change card sets positioned at the end of the file.

The delimiter used to separate the change card sets is a period (.) in column one. The end of the file is also signified by a period in column one. All of the CHEM_MACCS User Input Files must end with a period in column one. The sample EARL $\bar{Y}$ User Input File listed in Appendix D is an example illustrating the use of change cards for a PRA application of the code.

The purpose of the change card processing in EARLY is solely to allow modification of the previously specified emergency response scenario data. Any cards appearing in the change card sets must have been previously defined in either the Evacuation Zone Data (Section C.6) or the Shelter and Relocation Data (Section C.7). If data items from another data block appear in the change cards, they will be ignored. Each set of change cards must include a new value of EANAM2, a text field describing the emergency response scenario. Also, each set of change cards must produce a change in at least one of the numeric input variables described in the Evacuation Zone Data or in the Shelter and Relocation Data.

EARLY calculates the doses and health effects that result from exposure during the emergency response period for up to three different emergency response scenarios and these are written in binary form to units 31 to 33 . All of these files have the same format.

Whenever results are combined by the code, the listing produced by the OUTPUT module will present the overall combination of results as well the individual components from which it is constructed. 
It is up to the user to specify the parameters needed for these calculations. There are no default values. In addition to specifying the characteristics of the model, the user has complete control over the output produced by EARLY and must explicitly specify which results are to be produced. All of this information is supplied through the User Input File for EARLY and all of the input parameters are described in this appendix.

The User Input File for EARLY consists of ten data blocks used to define the simulation and an additional eight data blocks used to specify the output desired by the user. These eighteen data blocks are described below.

\section{C.2 Miscellaneous (Mm) Data}

The user must supply information to identify the run, define the histogram approximating the crosswind Gaussian distribution, and also supply information describing the handling of the wind rose. It is possible to specify a single wind rose that will override the wind roses that were calculated in ATMOS for each of the weather category sampling bins. The user must also specify how to treat changes in wind direction. There are three options to choose from and these are described below.

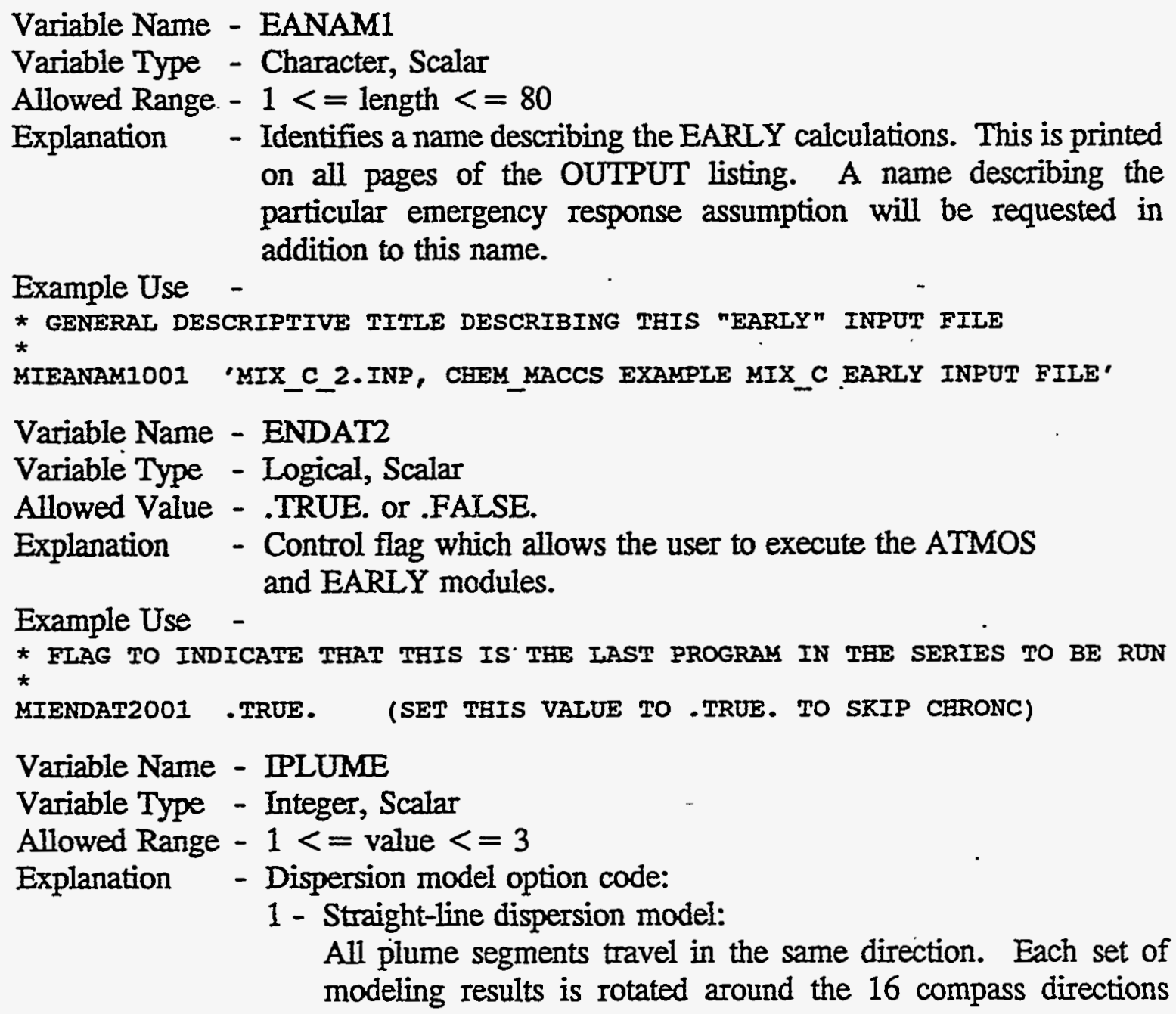
on all pages of the OUTPUT listing. A name describing the particular emergency response assumption will be requested in addition to this name.

Example Use -

* GENERAL DESCRIPTIVE TITLE DESCRIBING THIS "EARLY" INPUT FILE 
Example Use -

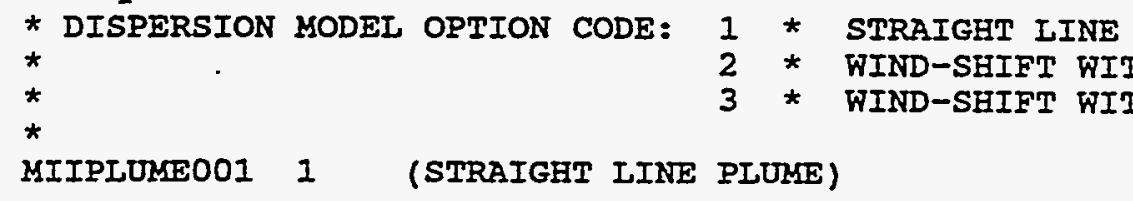$$
\text { * }
$$

MIIPLUMEOOI 1 (STRAIGHT IINE PIURE)

Variable Name - NUMFIN

Variable Type - Integer, Scalar

Allowed Range - 3, 5, or 7

Explanation - Number of fine grid subdivisions used by the model. A step function is used to approximate the Gaussian distribution of the plume in the crosswind direction. Each of the 22.5-degree sectors is subdivided into NUMFIN fine grid elements, with doses and risks being uniform in each of the fine grid elements.

Example Use -

* NUMBER OF FINE GRID SUBDIVISIONS USED BY THE MODEL.

MINOMFINOOI $7,(3,5$ OR 7 ALLOWED)

Variable Name - IPRINT

Variable Type - Integer, Scalar

Allowed Range $-0<=$ value $<=10$

Explanation - Specifies the quantity of debug output that is desired. The higher the value, the more output will be printed. Debug output is written to FORTRAN unit 6 (TAPE6). Normal runs should specify a value of zero. The choice of any other value should only be made by people familiar with the code for examination in detail of single weather sequences.

For various values of IPRINT, the code will print out intermediate results on the list output file. These are described below. (Groundshine is not used in CHEM_MACCS.)

IPRINT $\geq 1$ : skin dose conversion factors, centerline doses for all organs (if IPLUME =1), Gaussian histogram and cloudshine correction factors, return code values (RETCOD). 
IPRINT $\geq 2$ : final groundshine dose rate for each organ, each plume segment.

IPRINT $\geq 4$ : total acute dose for organs 2 and 3 , early fatality, early injury, and cancer risk values for each spatial element.

IPRINT 28: acute dose to organs 2 and 3 after completion of subroutine RELZON, acute dose to organs 2 and 3 after completion of subroutine ESTAT.

Example Use -

* LEVEL OF DEBUG OUTPUT REQUIRED, NORMAL RUNS SHOULD SPECIFY ZERO

*

MIIPRINTOO1 0 (TURN OFF THE DEBOG PRINT)

Variable Name - RISCAT

Variabie Type - Logical, Scalar

Allowed Value - .TRUE. or .FALSE.

Explanation - If the option of weather category bin sampling was chosen by the user in the ATMOS User Input File (METCOD=2), the display of results produced by the OUTPUT module can show the relative contribution

Example Use of each of the weather category bins to the mean consequence value.

* IOGICAT FLAG SIGNIFYING THAT THE BREARDOWN OF RISK BY WEATHER CATEGORY

* bin are to be pREsENTEd to show their relative contribution to the mean

*

* $\quad$ RISBIN

MIRISCATO01 .FAISE.

Variable Name - OVRRID

Variable Type - Logical, Scalar

Allowed Value - .TRUE. or .FALSE.

Explanation - Specifies whether the wind-rose probabilities are to be supplied by the user. If the weather sampling option was chosen in ATMOS (METCOD =2), wind roses for each weather sampling bin have been passed down from ATMOS. Those wind roses will be used if OVRRID $=$.FALSE. If no wind rose is available to EARLY, a uniform wind rose will be used, that is, $P=0.0625$ in each direction.

Example Use -

* FLAG INDICATING IF WIND-ROSES FROM ATMOS ARE TO BE OVERRIDDEN

MIOVRRIDOO1 .FALSE. (USE THE WIND ROSE CALCULATED FOR EACH WEATHER BIN)

Note to user: The following data are only required if (OVRRD=.TRUE.).

Variable Name - WINROS

Variable Type - Real, Array

Allowed Range - $0 .<=$ value $<=1$. 
Explanation - These are the probabilities of the wind blowing from the site into each of the 16 compass sectors (rotating clockwise from $N$ to NNW). The sum of these values must be between 0.95 and 1.05 . The user Example Use must supply 16 values in rows on one or more data cards.

* SITING STUDY WINDROSE FOR THE PEACH BOTTOM SITE

MIWINROSO01 8.521E-02 $6.360 \mathrm{E}-02 \quad 4.605 \mathrm{E}-02 \quad 5.189 \mathrm{E}-02 \quad 6.869 \mathrm{E}-02 \quad 9.493 \mathrm{E}-02$

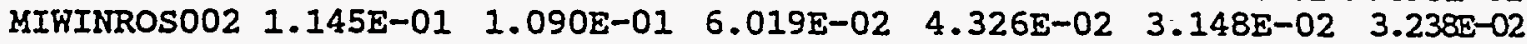

MIWINROSO03 $3.383 \mathrm{E}-02 \quad 4.625 \mathrm{E}-02 \quad 5.446 \mathrm{E}-02 \quad 6.424 \mathrm{E}-02$

\section{C.3 Population Distribution (PD) Data}

The user must supply information to define the polar coordinate population distribution surrounding the site. This information can be supplied from the Site Data File or a uniform population distribution can be specified by the user.

Note: Whatever values are supplied here will be used by the EARLY modules in defining the characteristics of the region surrounding the site.

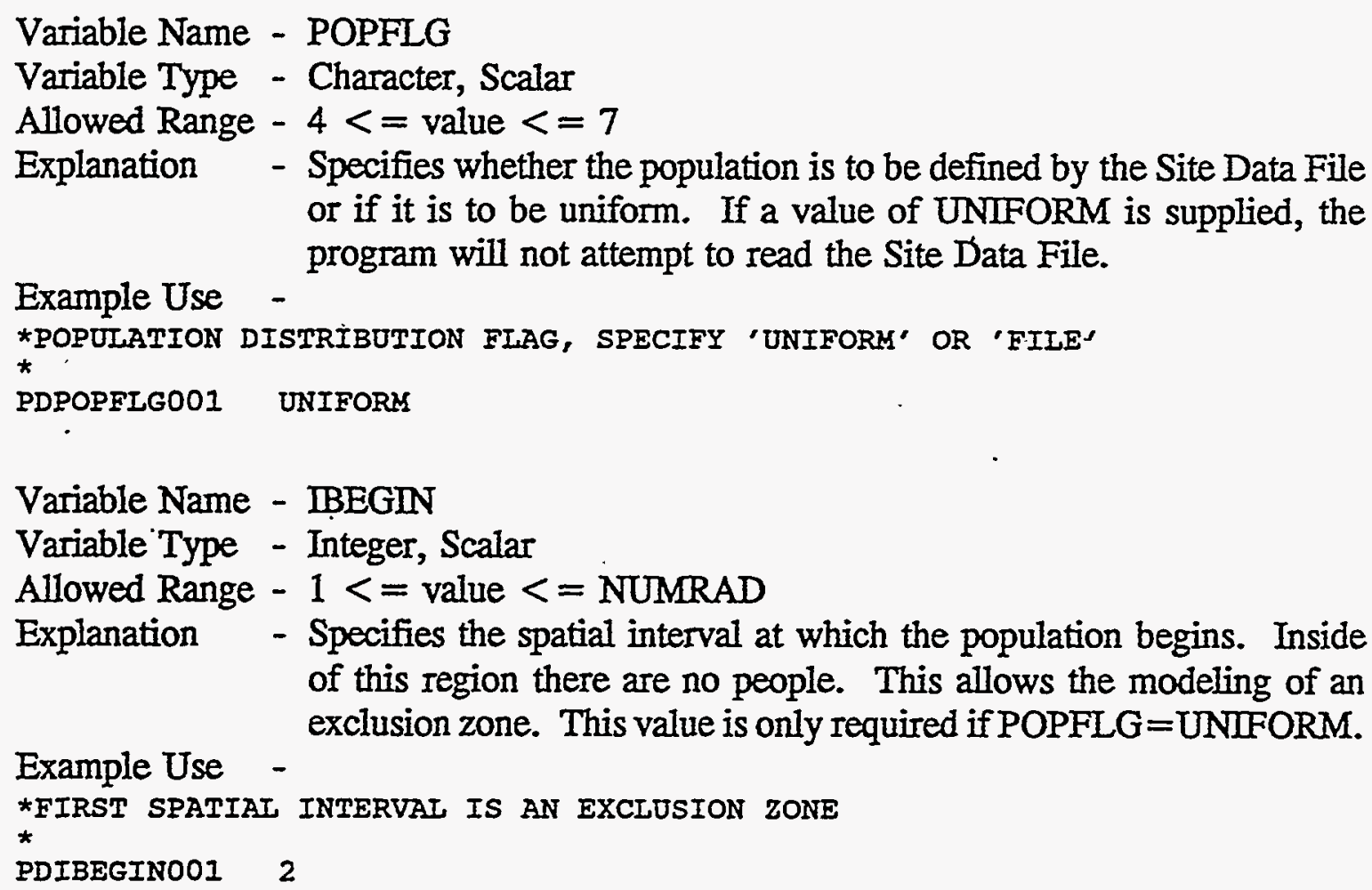
or if it is to be uniform. If a value of UNIFORM is supplied, the program will not attempt to read the Site Data File. of this region there are no people. This allows the modeling of an 


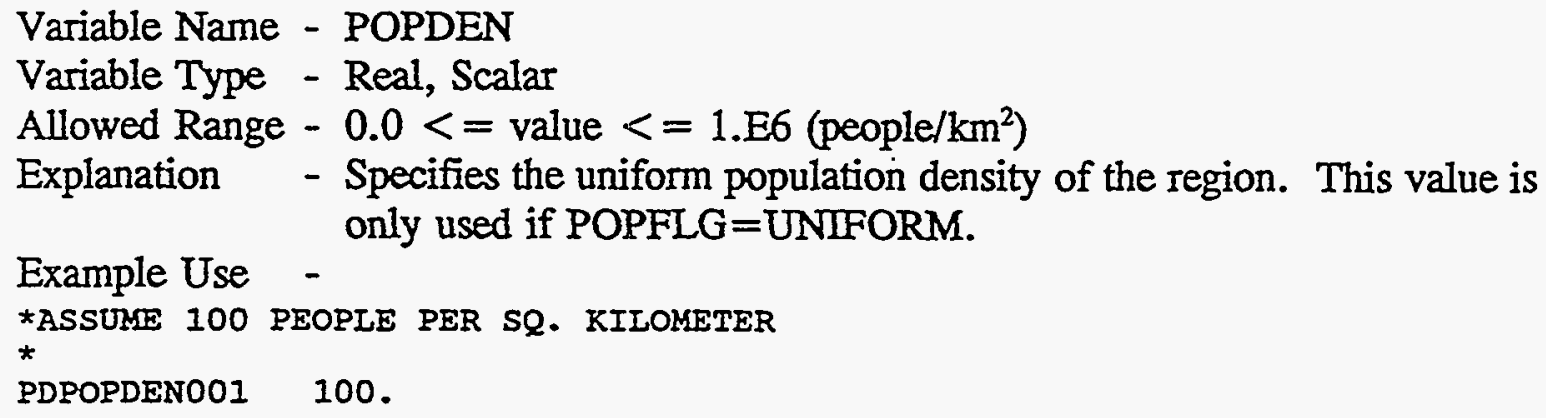

\section{C.4 Dose Definition (OD) Data}

The CHEM_MACCS code will only calculate doses defined in this section. The shelter and relocation model (variable CRIORG), and the health effects models (prompt fatalities, early injuries, and latent cancers) must only reference defined doses. The same is true of the dose-related output options (results of type 3, 5, and 6). MACCS will check to ensure that appropriate chemical species weighting factors are available on the DOSDATA.INP file for all of these doses.

MACCS can calculate two kinds of doses: lifetime dose and effective acute dose. Acute doses are used for calculating the acute health effects in the EARLY module. The acute health effects are "early fatalities" and "early injuries." Lifetime dose is utilized for calculating the cancer induction and population dose results. It represents the effective continuous daily dose associated with acute and intermediate-term exposures.

The list of doses for which concentration weighting factors are available is provided in the DOSDATA.INP file. The dose names must be spelled exactly they appear in the DOSDATA.INP file. If some other weighting factor file is used, its dose names must be identical to those input to EARLY in the ORGNAM list defined below. Any reference to a dose that cannot be found on the dose list (ORGNAM) will cause the error flag to be set and execution will be terminated.

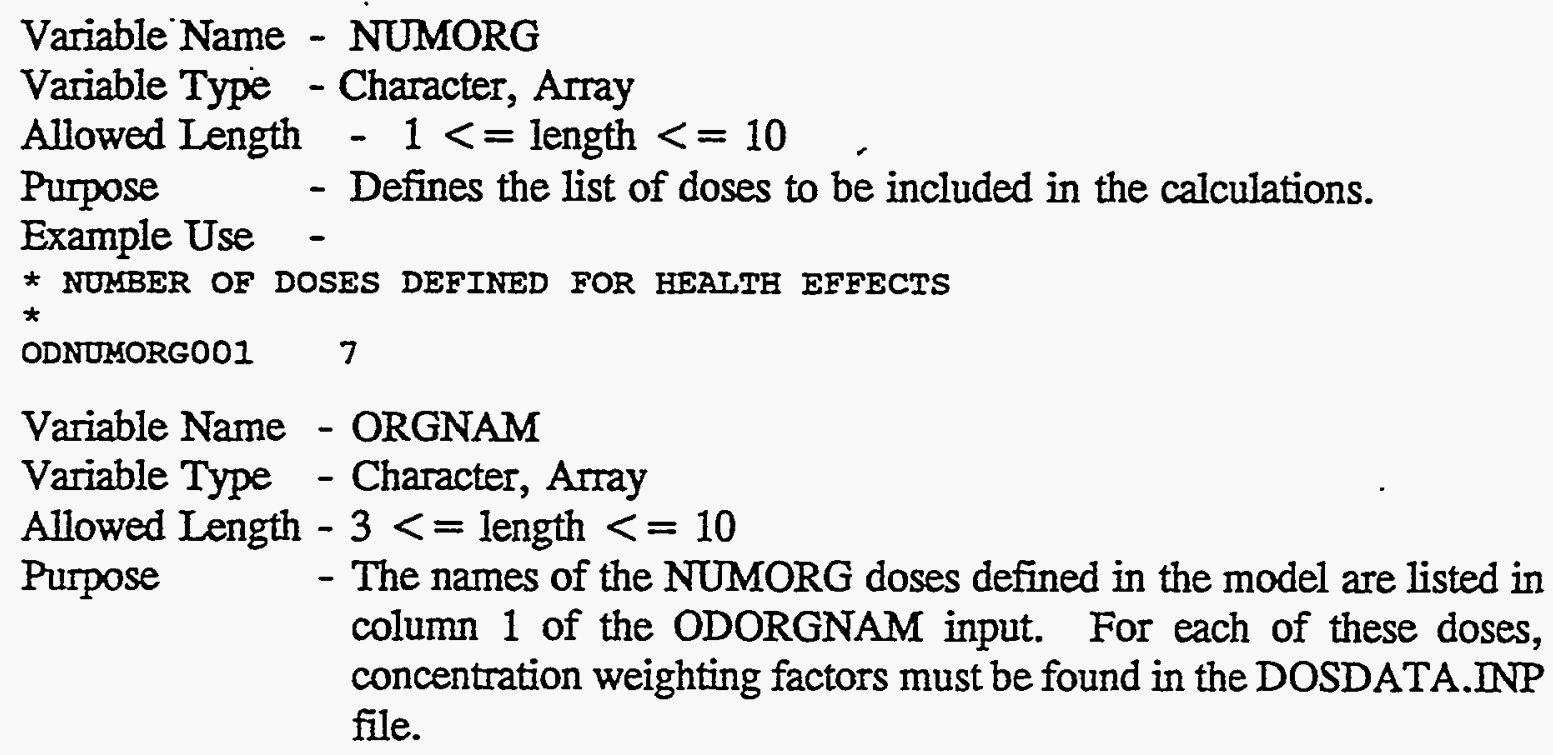
column 1 of the ODORGNAM input. For each of these doses, concentration weighting factors must be found in the DOSDATA.INP file. 
Variable Name - IPWAY

Variable Type - Character, Array

Allowed Length $-3<=$ length $<=10$

Purpose - The names of the pathways associated with each of the NUMORG doses defined in the model are listed in column 2 of the ODORGNAM input. Four pathways are allowed as illustrated below.

Variable Name - EXPN

Variable Type - Real, Array

Allowed Length $-0 .<=$ value $<=2$.

Purpose - The powers to which air concentrations are raised in calculating doses by the various pathways. For continuous daily doses, a value of 1 . should be used. NUMORG values should be provided in column 3 of the ODORGNAM input.

Example Use of ORGNAM, IPWAY, and EXPN .

* NAMES OF THE DOSES DEFINED FOR HEALTH EFFECTS

$\star$

*

ODORGNAMOOI

ODORGNAMOO2

ODORGNAMOO3

ODORGNAMOO 4

ODORGNAMOO5

ODORGNAMOO6

ODORGNAMOO7

\begin{tabular}{|c|c|c|}
\hline $\begin{array}{l}\text { DOSE } \\
\text { NAME }\end{array}$ & $\begin{array}{l}\text { PATHWAY } \\
\text { FOR DOSE }\end{array}$ & $\begin{array}{l}\text { CONCENTRATION } \\
\text { EXPONENT }\end{array}$ \\
\hline $\begin{array}{l}\text { 'VIN1GA' } \\
\text { 'VSK2GA' } \\
\text { 'ISK3GA' } \\
\text { 'ISK4GA' } \\
\text { 'VNE5GA' } \\
\text { 'VSK6GA' } \\
\text { 'VCDDGA' }\end{array}$ & $\begin{array}{l}\text { 'INH ACU' } \\
\text { 'CLD' } \\
\text { 'SKN ACU' } \\
\text { 'SKN ACU' } \\
\text { 'INH ACU' } \\
\text { 'CLD' } \\
\text { 'INH IIE' }\end{array}$ & $\begin{array}{l}1.0 \\
1.0 \\
1.0 \\
1.0 \\
1.0 \\
1.0 \\
1.0\end{array}$ \\
\hline
\end{tabular}

INTERNAL TO CHEM_ MACCS

(IPWAY $=5$ )

(IPWAY $=1$ )

(IPWAY $=-1$ )

(IPWAY $=-1$ )

(IPWAY $=5$ )

$(I P$ WAY $=1)$

$($ IPWAY $=6$ )

\section{C.5 Shielding and Exposure (SE) Data}

This section defines the shielding factors for exposure to inhalation and deposition to skin for three types of activities (normal activity, evacuation, and sheltering). A breathing rate is also specified for each type of activity. In addition, the resuspension parameters to be used for the emergency phase time period (EARLY), the resuspension coefficient and resuspension half-life are also defined.

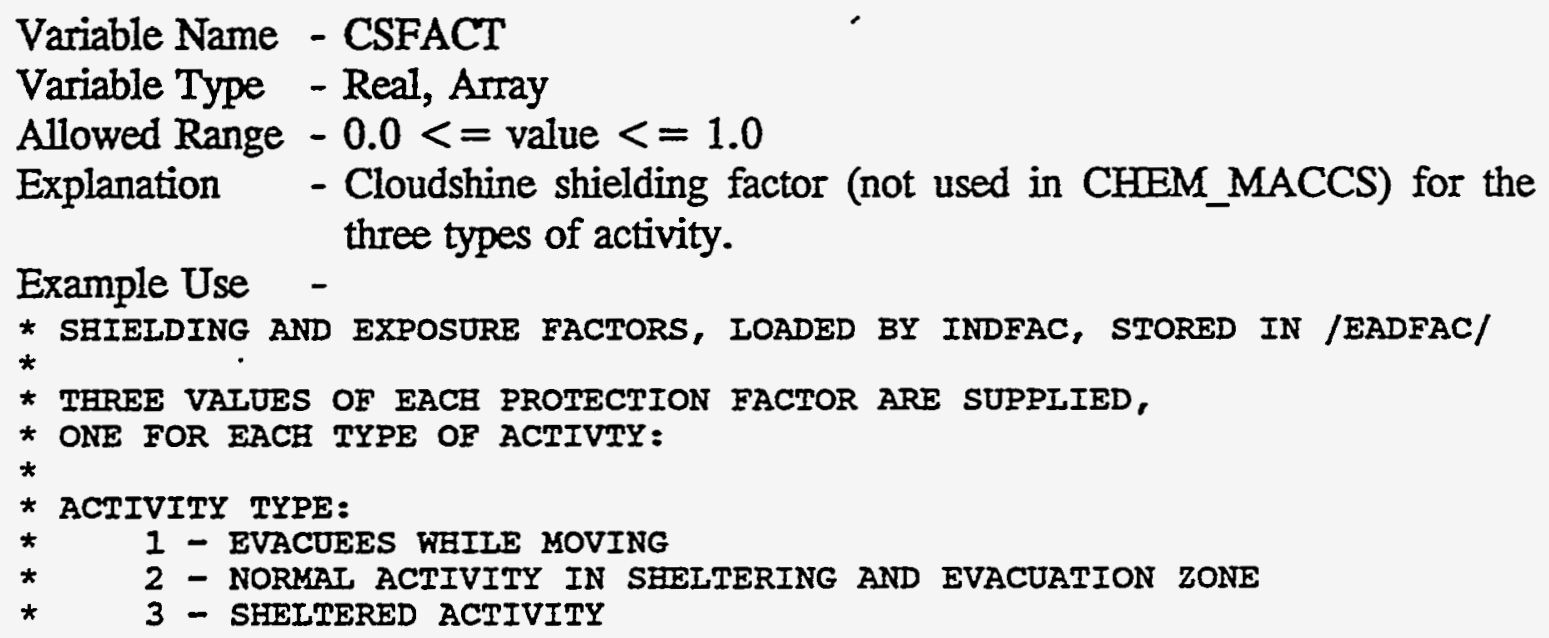
three types of activity.

Example Use -

* SEIELDING AND EXPOSURE FACTORS, LOADED BY INDFAC, STORED IN /EADFAC/

* three VAIUES of each pRotection factor are supplied,

* ONE FOR EACH TYPE OF ACTIVTY:

$*$

* ACTIVITY TYPE:

* 1 - EVACUEES WeIIE MOVING

* 2 - NORMAI ACTIVITY IN SHELTERING AND EVACUATION ZONE

* 3 - SHELTERED ACTIVITY 

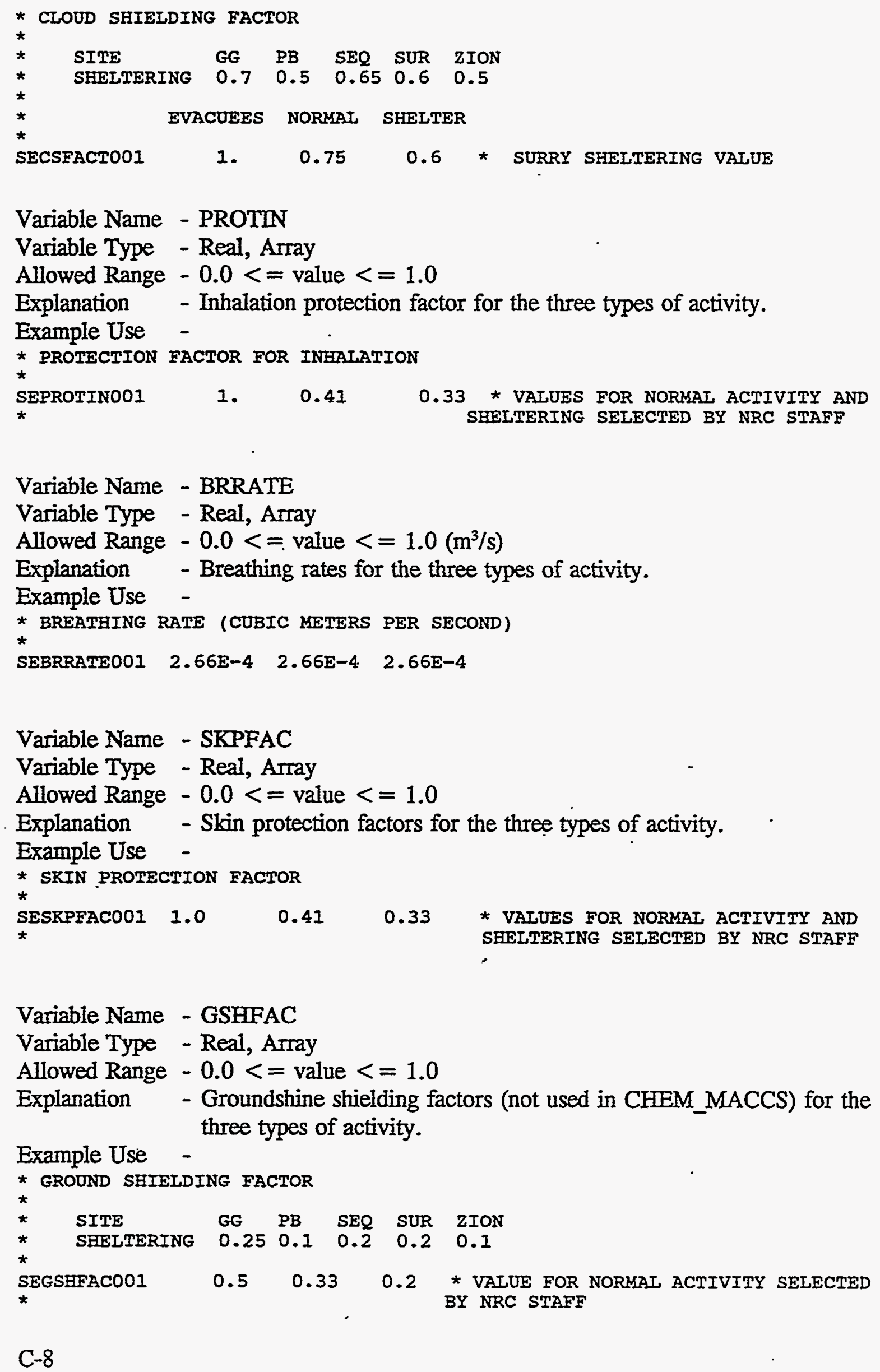


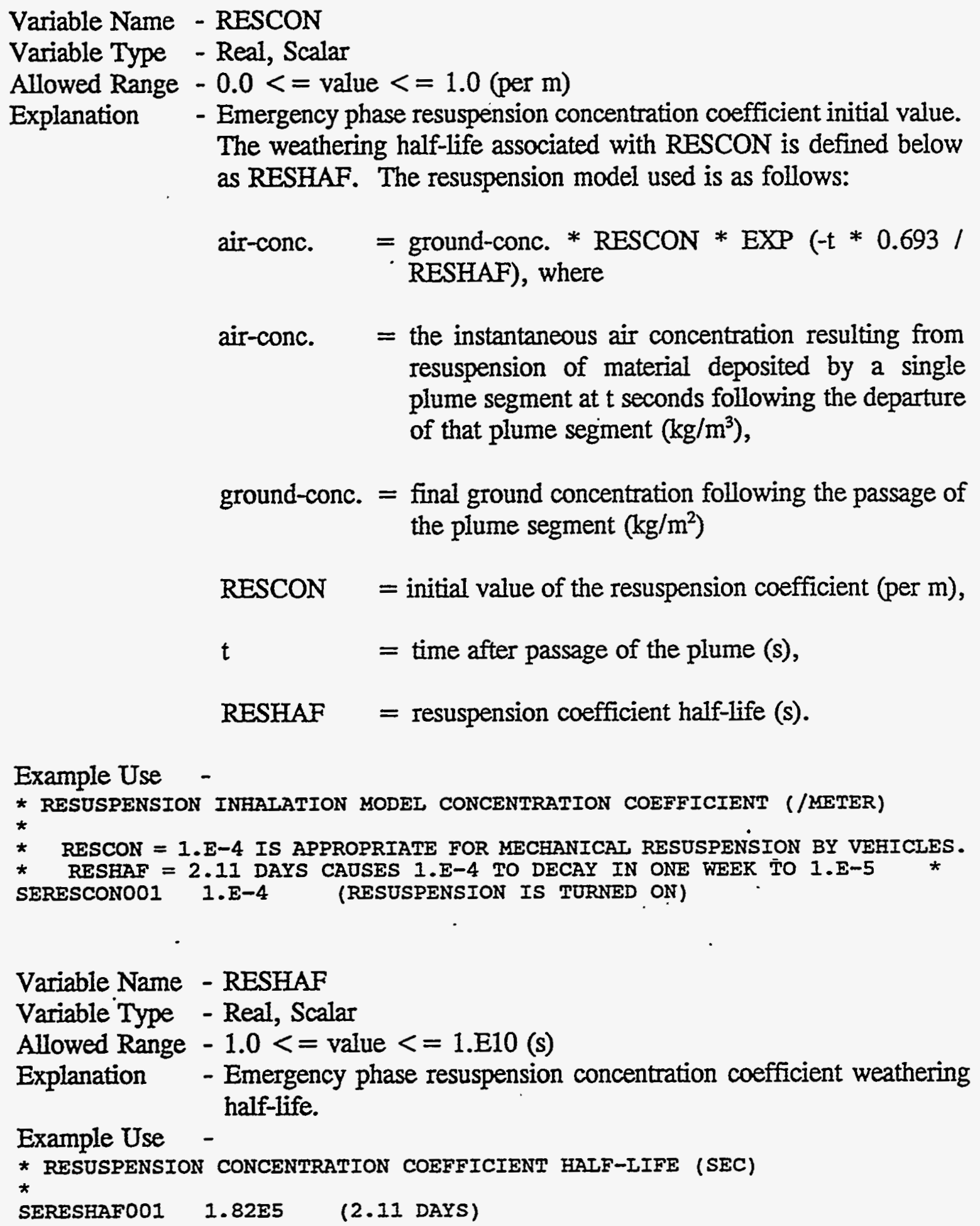

Variable Name - RESHAF

Variable Type - Real, Scalar

Allowed Range $-1.0<=$ value $<=1$. E10 (s)

Explanation - Emergency phase resuspension concentration coefficient weathering half-life.

Example Use -

* RESUSPENSION CONCENTRATION COEFFICIENT HALF-IIFE (SEC)

*

SERESHAFOO1 $1.82 E 5 \quad(2.11$ DAYS $)$ 


\section{C.6 Evacuation Zone (EZ) Data}

In the current implementation, CHEM_MACCS is limited to an evacuation model where the only movement allowed is radially outward.

All individuals between the first and last evacuating intervals [INIEVA and LASEVA(3)] are assumed to evacuate. Evacuating individuals travel radially outward at a constant speed and are subject to exposure until they leave the LSAMOV spatial interval, whereupon they are assumed to avoid all further exposure.

Within the evacuation zone, up to three regions with different evacuation delay times may be defined. The three times associated with these regions are specified in EDELAY. Outer boundaries of the three evacuation rings are specified in LASEVA. Some of these values may be zero, indicating that the region is null.

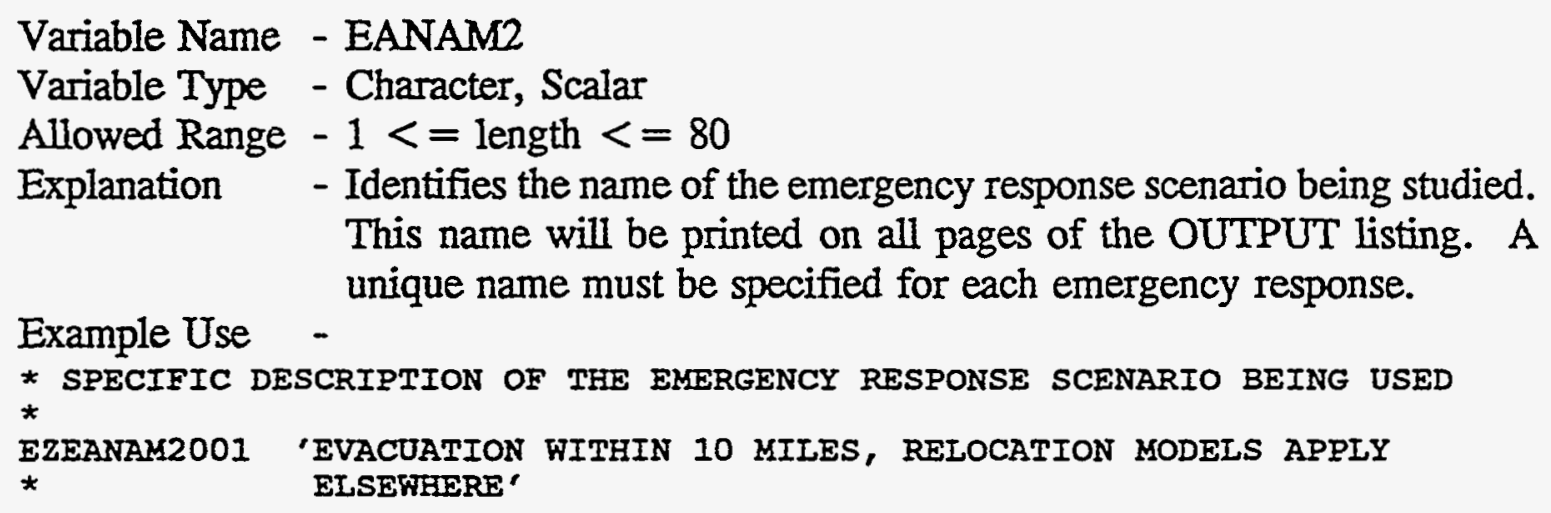
This name will be printed on all pages of the OUTPUT listing. A

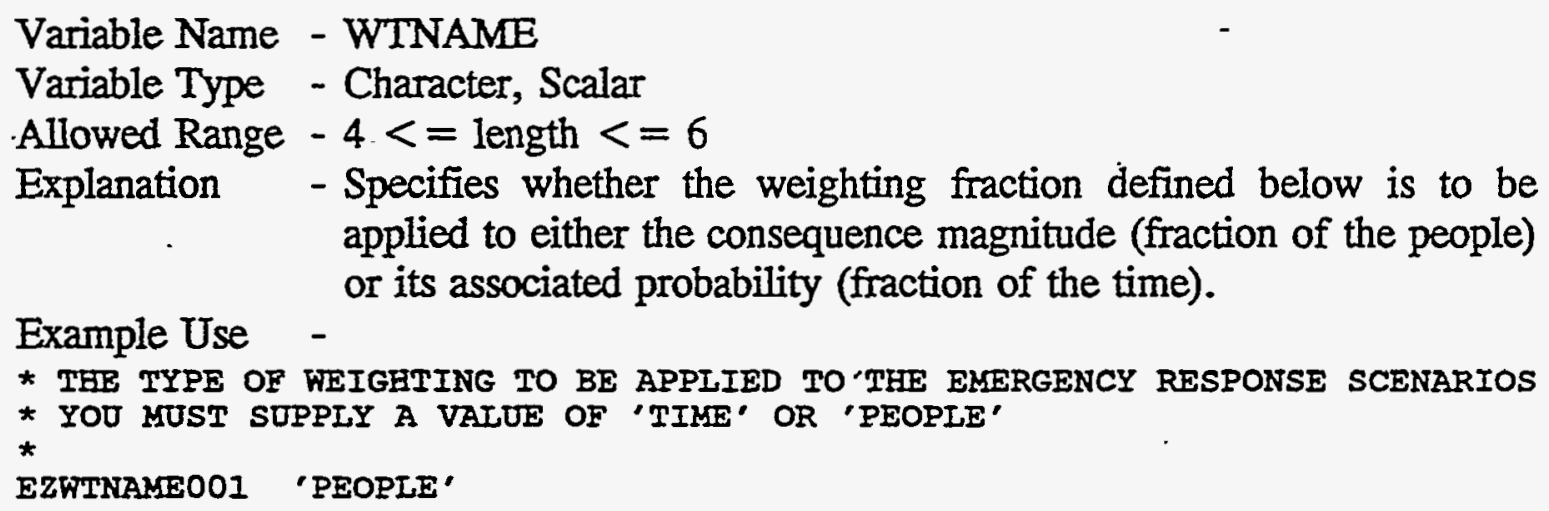
applied to either the consequence magnitude (fraction of the people) response scenario. This value is used by the OUTPUT module in combing results for the "overall weighting sum." The weighting can be done for either "fraction of the people" or "fraction of the time" as determined by the input variable WTNAME 
Example Use
* WEIGHTING FRACTION APPLICABLE TO THIS SCENARIO
*
EZWTFRACOO1 0.95

Variable Name - LASMOV

Variable Type - Integer, Scalar

Allowed Range $-0<=$ value $<=$ (NUMRAD - 1)

Explanation - The outermost spatial interval of the evacuation movement zone. This is the distance after which evacuees are assumed to disappear from the early health effects model and receive no further dose. If the user specifies a value of zero, there will be no evacuation and no

Example Use further data in this section are required.

* LAST RING IN THE MOVEMENT ZONE

*

EZLASMOVO01 15 (EVACUEES DISAPPEAR AFTER TRAVEIING TO 20 MILES)

Note to user: The following items in this section are only required if LASMOV is greater than zero.

Variable Name - INIEVA

Variable Type - Integer, Scalar

Allowed Range $-1<=$ value $<=$ LASMOV

Explanation - This value is only required if LASMOV is greater than zero. It specifies the innermost spatial interval of the evacuation zone. A value greater than one means that there will be an inner shelter zone. The inner shelter zone is composed of the INIEVA=1 spatial intervals closest to the facility.

Example Use -

* First spatial INTERVAI IN the eVacuation zoNe

EZINIEVAO01 1 (N,O INNER SHELTER ZONE)

Variable Name - LASEVA

Variable Type - Integer, Array

Allowed Range $-0<=$ value $<=$ LASMOV

Explanation - The outer spatial intervals marking off the three evacuation delay zones from the nearest to farthest. These allow a phased evacuation. The user must supply three values. A value of zero for any of the rings causes that zone to be null. The nonzero values must not be

Example Use less than INIEVA and they must be in ascending order.

* OUTER BOUNDS ON 3 EVACUATION ZONES (ZERO MEANS THE ZONE IS NOT DEFINED) *

EZLASEVA001 00012 (SINGLE EVACUATION ZONE OUT TO 10 MILES) 
Variable Name - EDELAY

Variable Type - Real, Array

Allowed Range -0 . $<=$ value $<=86400.0$ (s) (one day)

Explanation - Defines the time necessary for evacuees to get moving. Before evacuating, evacuees can receive doses from the plume segments while continuing normal activity. The user must supply evacuation delay times for each of the three evacuation rings even if some of them are null. Evacuees remain in place until EDELAY seconds have elapsed from the offsite alarm time, OALARM, (see Section B.11) and then they begin their evacuation.

Note: The reference point for evacuation (alarm time) is different from the reference time for relocation actions (time after first plume arrival).

Example Use -

* eVACUATION DELAY TIMSS FOR THE 3 EVACUATION zONES

* THIS IS THE DEIAY TIME FROM OALARM (ATMOS) TO WHEN PEOPLE START MOVING

*

EZEDELAYOO1 O. D. 7200. (SURRY)

Variable Name - ESPEED

Variable Type - Real, Scalar

Allowed Range $-0.1<=$ value $<=1$. E6 $(\mathrm{m} / \mathrm{s})$

Explanation - The speed at which all evacuees travel radially outward.

Example Use -

* RadiaI eVAcuation SPEEd ( $\mathrm{H} / \mathrm{S}$ )

$\star$

EZESPEEDOOI $1.8 \quad$ (SURRY)

\section{C.7 Shelter and Relocation (SR) Data}

The user can specify the existence of two shelter zones: one inside of the evacuation zone, the other outside of it. Both of these zones are optional. The inner shelter zone-is defined if a value greater than one was specified for INIEVA. The outer sheiter zone is defined if LASHE2 is assigned a nonzero value. Even though these zones are optional, the code requires that all of the parameters described in this section be supplied.

Variable Name - TTOSH1

Variable Type - Real, Scalar

Allowed Range $-0.0<=$ value $<=86400.0$ (s) (one day)

Explanation - Defines the time to take shelter in the inner shelter zone. People here take sheiter TTOSH1 seconds after the off-site alarm time, OALARM(see Section B.11). Normal activity shielding factors are used up to the time at which individuals take shelter. Upon taking shelter, the shelter shielding factors are used.

Note: The reference point for sheltering (alarm) is different from reference time for relocation actions (first plume arrival).

Example Use -

* TIHE TO TAKE SHELTER IN THE INNER SHELTER ZONE (SECONDS FROM OAIARRM)

SRTTOSH1001 O. (TEERE IS NO INNER SHELTER ZONE) 


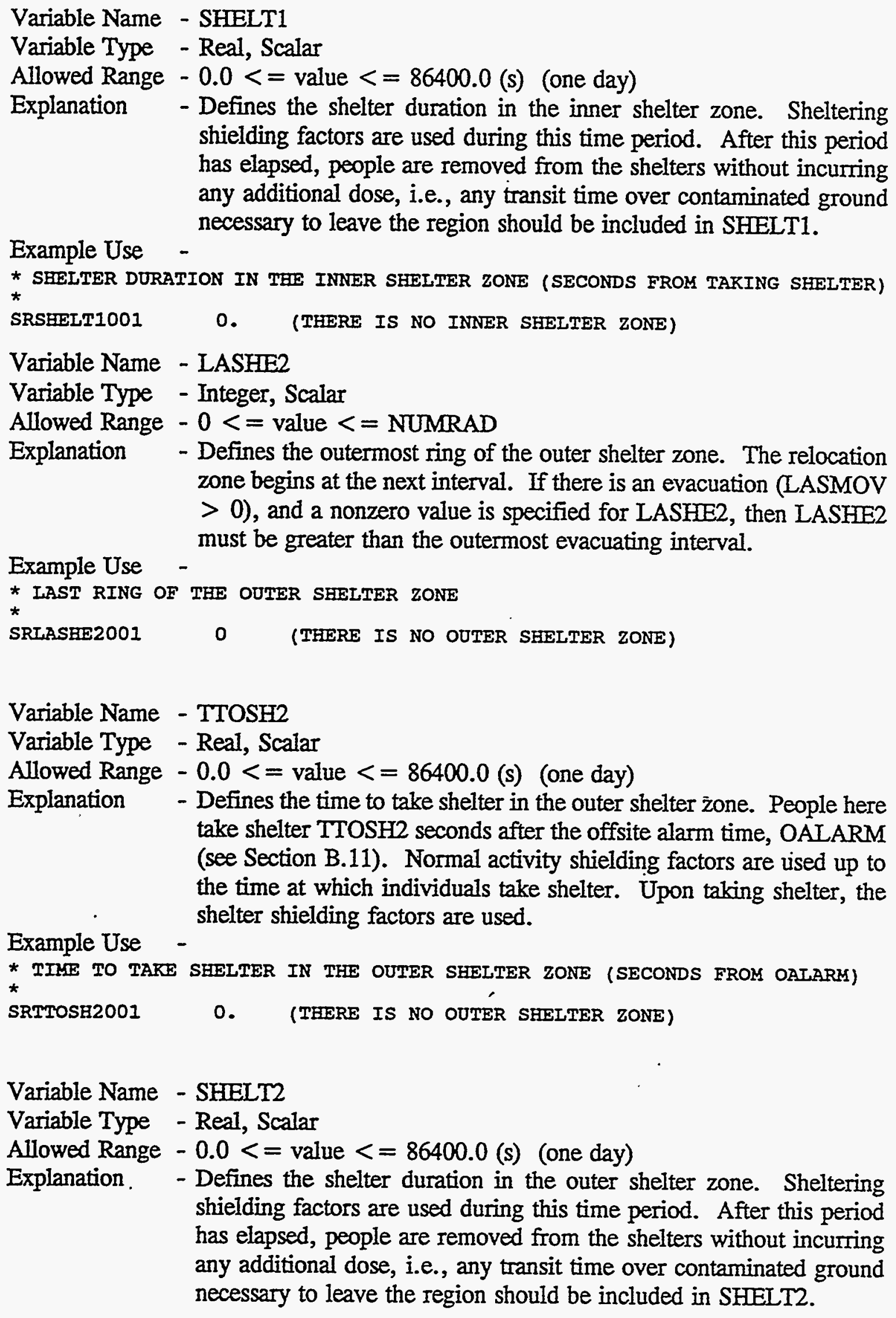
shielding factors are used during this time period. After this period has elapsed, people are removed from the shelters without incurring any additional dose, i.e., any transit time over contaminated ground zone begins at the next interval. If there is an evacuation (LASMOV $>0$ ), and a nonzero value is specified for LASHE2, then LASHE2 take shelter TTOSH2 seconds after the offsite alarm time, OALARM (see Section B.11). Normal activity shielding factors are used up to the time at which individuals take shelter. Upon taking shelter, the shielding factors are used during this time period. After this period has elapsed, people are removed from the shelters without incurring any additional dose, i.e., any transit time over contaminated ground necessary to leave the region should be included in SHELT2. 
Example Use -

* SHELTER DURATION IN THE OUTER SHELTER zONE (SECONDS FROM TAKING SHELTER)

SRSHELT2001 0 . (THERE IS NO OUTER SHELTER ZONE)

Variable Name - ENDEMP

Variable Type - Real, Scalar

Allowed Range $-86400.0<=$ value $<=604800.0$ (seconds after plume arrival)

Explanation - Defines the duration of the emergency phase period. EARLY only calculates doses that would be received during the emergency phase time period. Doses at each spatial interval are cut off at ENDEMP seconds after the arrival of the first plume segment to reach the interval. This cutoff applies to all individuals, no matter where they are located.

ENDEMP is also the duration for which evacuees and shelterees are kept away from their homes if there was any contamination in the

Example Use coarse grid element in which they reside.

* DURATION OF THE EMERGENCY PHASE (SECONDS FROM PLUME ARRIVAI)

SRENDEMPOO1 604800. (ONE WEEK)

Variable Name - CRIORG

Variable Type - Character, Scalar

Allowed Range $-2<=$ length $<=10$

Explanation - Defines the critical organ for relocation decisions during the emergency phase period considered by EARLY. In order to determine whether people can remain in the relocation zone, the total committed dose to the critical organ of an individual who remained in place for the entire emergency phase is calculated. The critical organ must be found on the list of organs, ORGNAM, defined in Section C.4.

Example Use -

* CRITICAL ORGAN FOR RELOCATION DECISIONS

*

SRCRIORG001 'VINIGA'

Variable Name - TIMHOT

Variable Type - Real, Scalar

Allowed Range $-0.0<=$ value $<=$ ENDEMP (seconds after plume arrival)

Explanation - Defines the hot spot relocation action time. Hot spot relocation can only occur for individuals residing outside of the emergency response zone. That is, doses to people awaiting evacuation or protected in shelters will not be affected by the hot spot relocation model.

If the total dose commitment to any individual in a coarse grid element in the relocation region would exceed DOSHOT to the critical organ, CRIORG, for someone remaining there for the entire 
emergency phase (ENDEMP), people in that element are relocated at TIMHOT seconds after the arrival of the first plume at that distance.

For the purpose of evaluating the need for hot spot and normal relocation, the total dose commitment is the dose which would be received by an individual who remained in place for the entire emergency phase period while engaging in "normal" activity. The pathways used for calculating the total dose commitment are direct inhalation and resuspension inhalation.

Any individuals relocated due to hot spots are removed from the problem for the duration of the emergency phase and receive no additional dose during the emergency phase.

Example Use

* HOT SPOT RELOCATION TIME (SECONDS FROM PLUME ARRIVAL)

SRTIMHOTO01 43200. (ONE-HALF DAY)

Variable Name - TIMNRM

Variable Type - Real, Scalar

Allowed Range - TIMHOT $<=$ value $<=$ ENDEMP (seconds after plume arrival)

Explanation - Defines the normal relocation action time. Normal relocation can only occur for individuals residing outside of the emergency response zone. That is, doses to people awaiting evacuation or protected in shelters will not be affected by the normal relocation model.

If the total dose commitment to any individual in a coarse grid element in the relocation region would exceed DOSNRM to the critical organ, CRIORG, for someone remaining there for the entire emergency phase (ENDEMP), people in that element are relocated at TIMNRM seconds after the arrival of the first plume at that distance. Any individuals subject to normal relocation are removed from contaminated areas for the duration of the emergency phase and receive no additional dose during the emergency phase.

For the purpose of evaluating the need for hot spot and normal relocation, the total dose commitment is the dose which would be received by an individual who remained in place for the entire emergency phase period while engaging in "normal" activity. The pathways used for calculating the total dose commitment are direct inhalation and resuspension inhalation.

Example Use -

* NORMAL RELOCATION TIME (SECONDS FROM PIUME ARRIVAI)

SRTIMNRMO01 86400. (ONE DAY) 


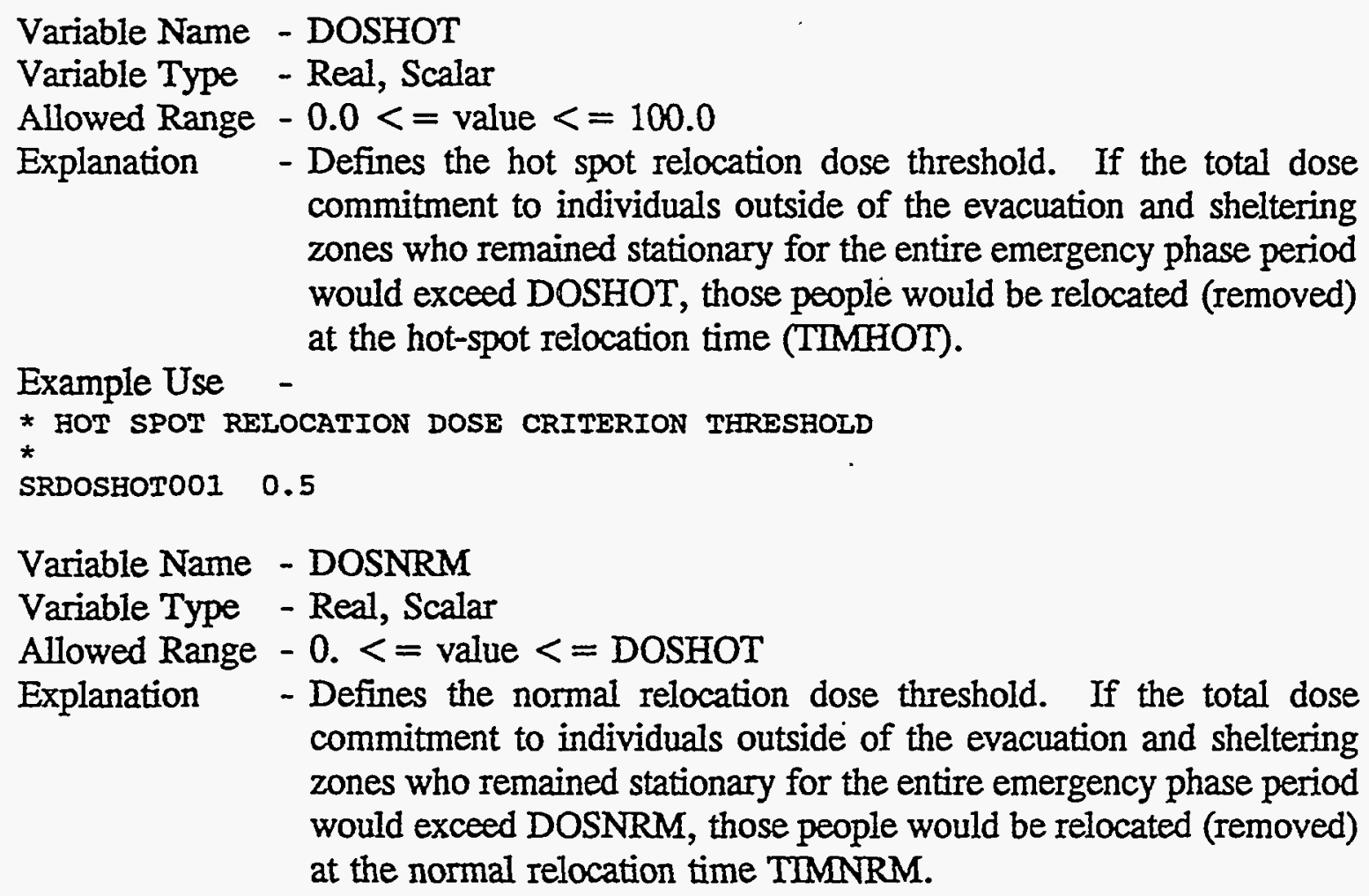
commitment to individuals outside of the evacuation and sheltering zones who remained stationary for the entire emergency phase period would exceed DOSHOT, those people would be relocated (removed) commitment to individuals outside of the evacuation and sheltering zones who remained stationary for the entire emergency phase period would exceed DOSNRM, those people would be relocated (removed) at the normal relocation time TIMNRM.

Example Use -

* NORMAI RELOCATION DOSE CRITERION THRESHOLD SRDOSNRMOOI 0.045

\section{C.8 Early Fatality (EF) Data}

Information in this section is only used to control the calculation of individual risk. Results to be processed by OUTPUT, e.g., total cases of early fatality, average individual risk of early fatality, and centerline risk versus distance of early fatality, are described in later sections of this document. In order for the code to produce early fatality results, the early fatality model must be defined in this. section.

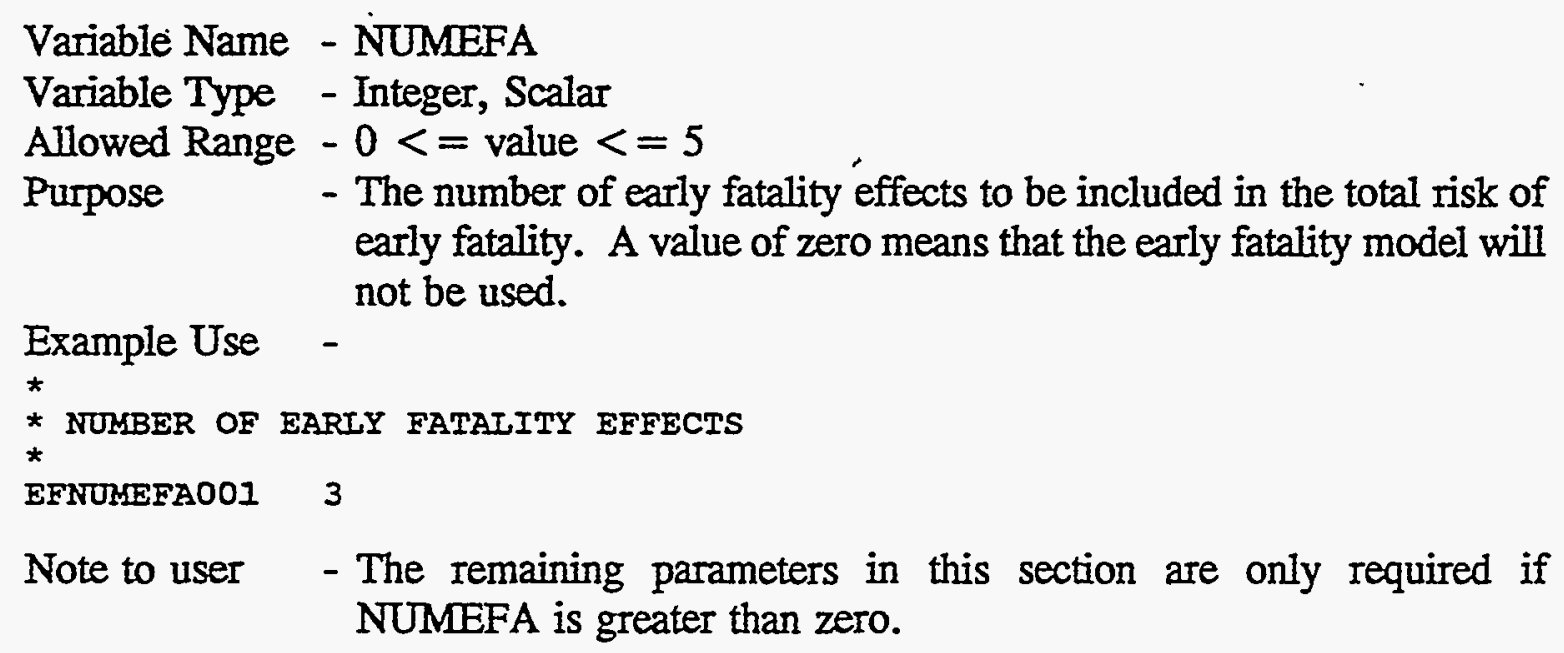
early fatality. A value of zero means that the early fatality model will not be used.

Example Use -

*

* nUmber of eariy fatatity efFects

* EFNUMEFAOO1 3

Note to user - The remaining parameters in this section are only required if NUMEFA is greater than zero. 


\begin{abstract}
Variable Name - ORGNAM
Variable Type - Character, Array

Allowed Range $-2<=$ length $<=10$

Purpose - The name of the dose for each early fatality effect. The dose must be one of those listed in the dose definition data. The user must supply NUMEFA values in column one of the data block.
\end{abstract}

Variable Name - EFFACA

Variable Type - Real, Array

Allowed Range $-1.0<=$ value $<=100.0$

Purpose

- The alpha factor (LD50) in the hazard function (probit equation) for the early fatality effect. The user must supply NUMEFA values in column two of the data block.

Variable Name - EFFACB

Variable Type - Real, Array

Allowed Range $-1.0<=$ value $<=100.0$

Purpose

- The Bliss slope in the hazard function (probit equation) for the early fatality effect. The user must supply NUMEFA values in column three of the data block.

Variable Name - EFFTHR

Variable Type - Real, Array

Allowed Range $-0.0<=$ value $<=100.0$

Purpose - The threshold dose below which the risk of early fatalities is presumed to be zero. If the acute dose falls below this threshold, it will not contribute to the risk of fatality. The user must supply NUMEFA values in column four of the data block.

Example Use of EFFACA, EFFACB, and EFFTHR -

\begin{tabular}{|c|c|c|c|c|}
\hline * & ORGNAM & EFFACA & EFFACB & EFFTHR \\
\hline $\begin{array}{l}\text { EFATAGRPO01 } \\
\text { EFATAGRPOO2 } \\
\text { EFATAGRPOO3 }\end{array}$ & $\begin{array}{l}\text { 'VIN1GA' } \\
\text { 'VSK2GA' } \\
\text { 'ISR3GA' }\end{array}$ & $\begin{array}{r}70.0 \\
15000.0 \\
1500.0\end{array}$ & $\begin{array}{r}12.0 \\
4.8 \\
4.8\end{array}$ & $\begin{array}{r}0.7 \\
99.0 \\
15.0\end{array}$ \\
\hline
\end{tabular}

\title{
C.9 Early Injury (En) Data
}

Information in this section is only used to control the calculation of early injury risks. Results to be processed by OUTPUT include total cases of a given injury, average individual risk of a given injury, and centerline risk versus distance of a given injury. In order for the code to produce early injury results, the injuries must be defined in this section.

The individual risk of each type of early injury is modeled in CHEM_MACCS using a probit equation analogous to those used for calculating early fatality risks. The early injury risk model differs from the early fatality model in that no composite risk of injury is calculated. The risk of each acute injury is presented separately. 
In addition to the parameters of the probit equations and the early injury dose thresholds, the user must specify the fraction of the population that is susceptible to each injury, EISUSC.

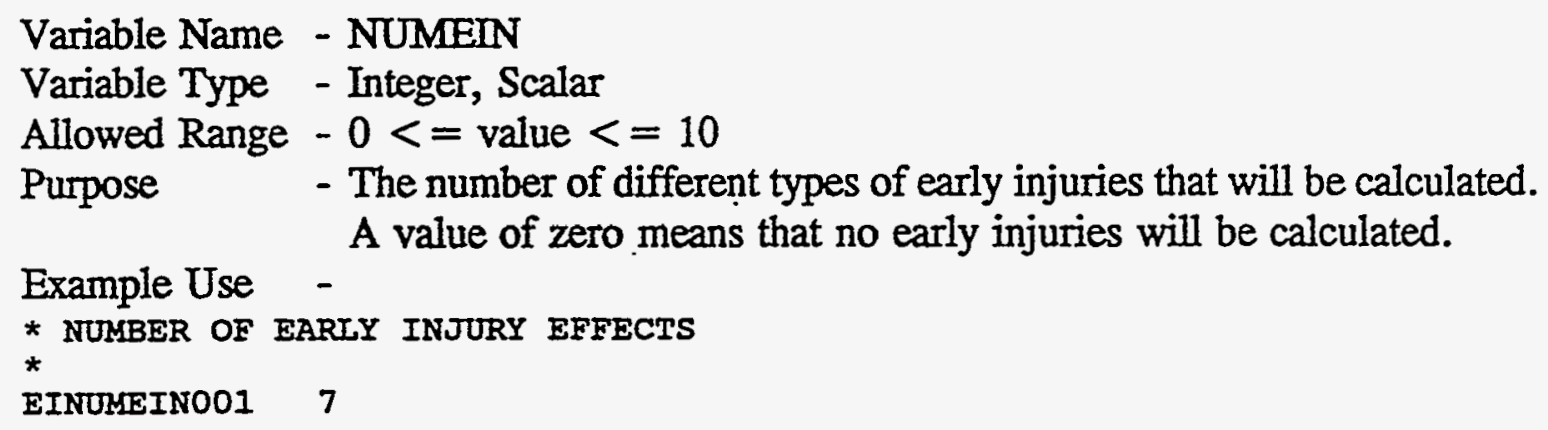

Note to user - The remaining parameters in this section are only required if NUMEIN is greater than zero. The six arrays are arranged as rows of data in a single data block.

Variable Name - EINAME

Variable Type - Character, Array

Allowed Range $-1<=$ length $<=16$

Purpose - The name of each type of early injury. The user may specify any name. Apostrophes are mandatory if there are any embedded blanks. The user must supply NUMEIN values in column one of the data block.

\section{Variable Name - ORGNAM}

Variable Type - Character, Array

Allowed Range $-2<=$ length $<=10$

Purpose $\quad-$ The name of the dose giving rise to each early injury. The early injury doses must be selected from those listed in the dose definition section (Section C.4). The user must supply NUMEIN values in column two of the data block.

Variable Name - EISUSC

Variable Type - Real, Array

Allowed Range $-0.0<=$ value $<=1.0$

Purpose

- The fraction of the population that is susceptible to the early injury. The user must supply NUMEIN values in column three of the data block.

Variable Name - EITHRE

Variable Type - Real, array

Allowed Range $-0.0<=$ value $<=1000.0$

Purpose

- The threshold dose below which the risk of the injury is zero. The user must supply NUMEIN values in column four of the data block. 


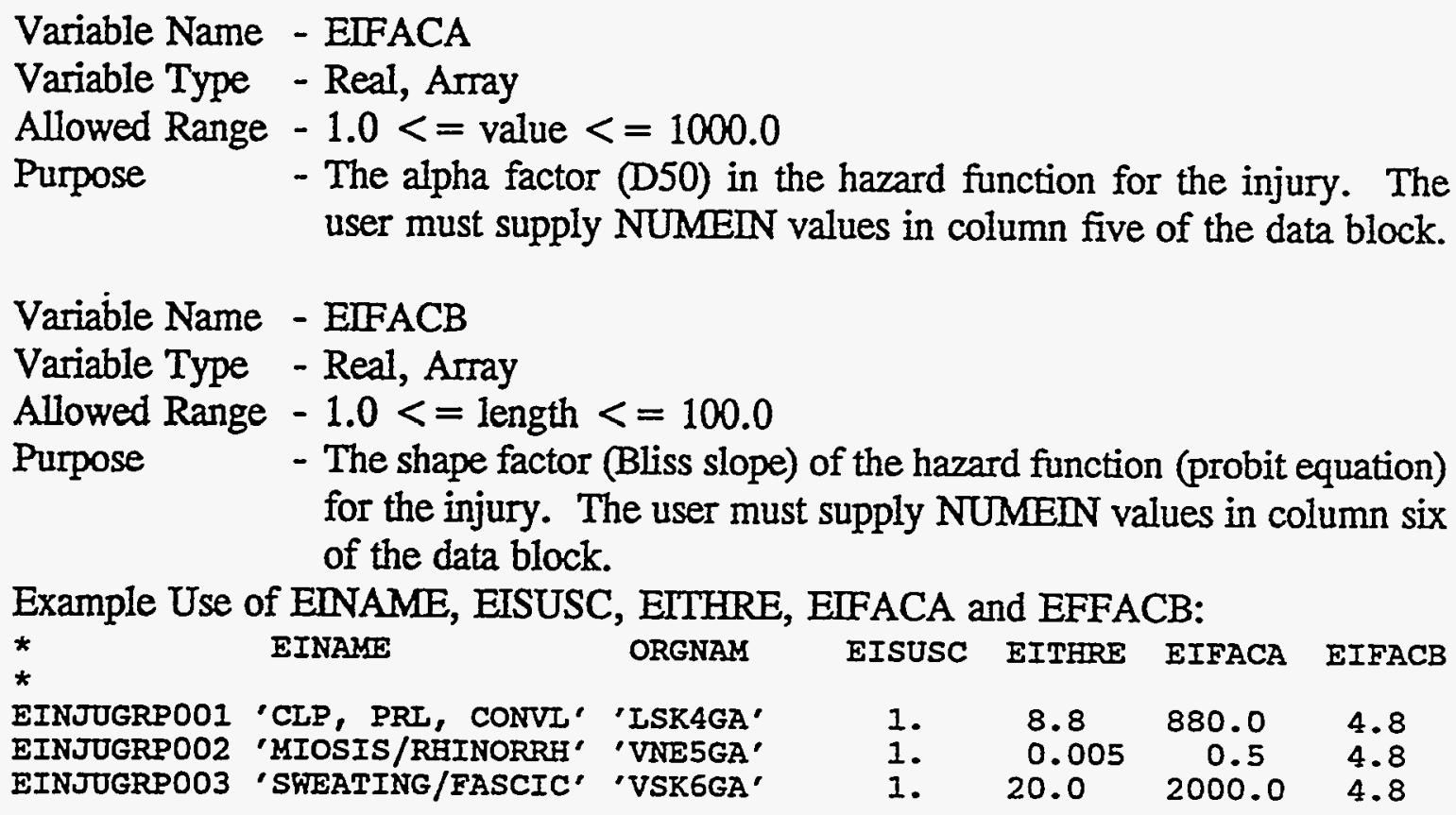

\section{C.10 Latent Cancer (LC) Data}

This section defines the cancer induction models used by the EARLY module. CHEM_MACCS calculates two types of dose: acute and lifetime. The acute dose is used soleiy for calculation of the nonstochastic effects (early fatalities and early injuries) associated with high exposures. The acute doses defined in CHEM_MACCS are not utilized in the cancer risk models. The lifetime dose used in the cancer risk model is an effective continuous daily dose.

In CHEM_MACCS, a linear dose-response relationship is used. The incremental risk of contracting cancer is the product of the effective continuous daily inhalation dose and the potency factor. At the time this was written, the only data on potency factors that were available were for mustard gas (HD), which is assigned a potency factor of 1.3. The other four chemical species treated by CHEM_MACCS (GA, GB, and VX) were assigned potency factors of 1.0. The potency factor ratios in the DOSDATA.INP files must be changed if other potency factor values are adopted.

Information in this section is only used to control the calculation of individual cancer risk. Results to be processed by OUTPUT, e.g., total cases of a given cancer, average individual risk of a given cancer, and centerline risk of a given cancer versus distance, are described in later sections. In order for the code to produce cancer results, the cancers must be defined in this section.

\footnotetext{
Variable Name - NUMACA

Variable Type - Integer, Scalar

Allowed Range $-0<=$ value $<=10$

Explanation - The number of different types of latent cancer effects that will be calculated. A value of zero means that latent cancer effects will not be calculated. Based on the current approach, NUMACA should be one for CHEM_MACCS.
} 
Example Use:

* number of cancer effects

ICNUMACAOO1 I

The remaining parameters in this section are only required if NUMACA is greater than zero.

Variable Name - ACTHRE

Variable Type - Real, Scalar

Allowed Range $-0.0<=$ value $<=10.0$

Explanation - The lower dose limit for the linear dose-response relationship. For doses less than ACTHRE, the linear-quadratic relationship is used. In CHEM_MACCS, a value of ACTHRE $=0.0$ should be used.

Example Use:

* DOSE TERESHOLD FOR APPLYING THE DOSE-DEPENDENT REDUCTION FACTOR

*

ICACTHREOOI 0.0

The next eight variables are supplied in a block of data as columns. An example of their use will follow.

Variable Name - ACNAME

Variable Type - Character, Array

Allowed Range $-1<=$ length $<=10$

Explanation - The name of each type of latent cancer effect. The user may specify any name. Apostrophes are mandatory if there are any embedded blanks. The user must supply NUMACA values in column one of the data block.

Variable Name - ORGNAM

Variable Type - Character, Array

Allowed Range $-2<=$ length $<=10$

Explanation - The name of the dose associated with each latent cancer effect. The dose must be one of those listed in the dose definition section (Section C.4). The user must supply NUMACA values in column two of the data block.

Variable Name - ACSUSC

Variable Type - Real, Array

Allowed Range $-0.0<=$ value $<=1.0$

Explanation - The fraction of the population that is susceptible to the latent cancer. The user must supply NUMACA values in column three of the data block. 

Variable Name - DOSEFA
Variable Type - Real, Array
Allowed Range $-0.0<=$ value $<=2.0$
Explanation - Defines the linear factor of the dose dependence in the cancer risk model. The user must supply NUMACA values in column four of the data block. In CHEM_MACCS, DOSEFA $=1.0$ should be input.
Variable Name - DOSEFB
Variable Type - real, array
Allowed Range $-0.0<=$ value $<=2.0$
Explanation - Defines the quadratic factor of the dose dependence in the cancer risk model which is used by the EARLY module. If the dose to the target organ is below ACTHRE, this parameter defines the quadratic term of the cancer dose-response function. The user must supply NUMACA values in column five of the data block. A value of DOSEFB $=0.0$ should be input for CHEM_MACCS.
Variable Name - CFRISK
Variable Type - real, array
Allowed Range $-0.0<=$ value $<=1.0$
Explanation - Lifetime risk factor for cancer death. Since the potency factors used in CHEM_MACCS are for cancer incidence, not cancer fatalities, CFRISK $\equiv 0.0$ should be input.

Variable Name - CIRISK

Variable Type - real, array

Allowed Range $-0.0<=$ value $<=1.0$

Explanation - Lifetime risk (potency) factor for cancer injury. The value specified should be for the reference chemical as indicated by the last two letters of the dose name. The user must supply NUMACA values in column seven of the data block.

Variable Name - DDREFA

Variable Type - real, array

Allowed Range $-1.0<=$ value $<=10.0$

Explanation - Dose-dependent reduction factor. If the lifetime dose commitment incurred during the EARLY exposure period is less than DDTHRE, the risk of cancer from irradiation of that organ is reduced by a factor of DDREFA. The user must supply NUMACA values in column eight of the data block. DDREFA $=1.0$ is appropriate for CHEM_MACCS.

Example Use:

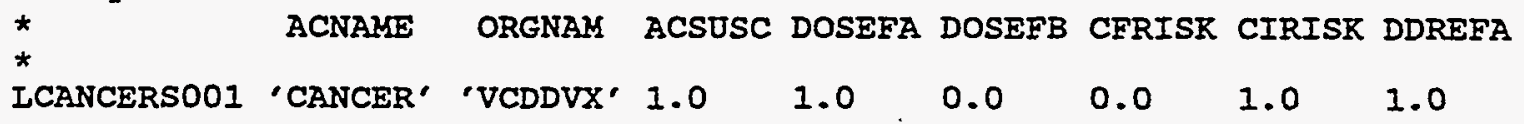




\section{C.11 Generation of Consequence Distributions}

Under the control of parameters supplied by the user on the EARLY User Input File, the EARLY module can calculate a variety of different consequence measures to portray the impact of a facility accident on the surrounding region.

The user has total control over the results that will be produced. By choosing appropriate values in the user input files, the user can ensure that the code does not perform unnecessary calculations.

This affords a great deal of flexibility but it also requires that the user anticipate which results will be of interest. If any are omitted, it is necessary to correct the user input and rerun the program.

In this regard, please remember that a result can only be produced if the model needed for its calculation has been previously defined in the appropriate section. If any results pertaining to health effects are requested, risk factors for that model must have been supplied in the sections entitled Early Fatality (EF), Early Injury (EI), and Latent Cancer (LC).

EARLY can produce ten different types of results. These are described in the next ten sections. EARLY does not generate Complementary Cumulative Distribution Functions (CCDFs) of the results that they calculate. Since EARLY generates the requested consequence measures, those numbers are written to binary files on units 31 to 34 for later processing into CCDFs.

The generation of CCDFs is performed by the OUTPUT module of CHEM_MACCS. It reads the binary files of consequence measures and automatically combines the results in a predetermined way. The user has no direct control over the OUTPUT module other than through the EARLY data blocks which control the generation of consequence measures.

The CCDF is an estimate of the distribution of consequence magnitudes. The variability of consequence values in CHEM_MACCS CCDFs is due solely to the uncertainty of the weather conditions existing at the time of the accident.

If a consequence measure is calculated by EARLY, the output module will present those results and their CCDFs. The contribution of up to three sets of results generated by EARLY can be combined according to the weighting fractions described in Section C.6. These weighting fractions can apply to either consequence magnitude (fraction of the people), or the consequence probability (fraction of the time). The weighted sum of all consequence measures, calculated by summation of results from separate runs of EARLY, are presented at the beginning of each section of the listing produced by OUTPUT. Following the overall weighted sum, the results from its individual components are presented. 
The following material describes the format of the listing produced by the OUTPUT module. It is recommended that the reader of this section refer to Appendix D, which lists the output from Sample Problem VX_A. This output file is named VX_AORI.OUT.

At the top of each page is printed the date and time of the run and a page number. The numbering of pages begins with the first page produced by the OUTPUT module.

The leftmost 38 columns of the page provide the name of the consequence measure. These names are split into two parts: the general and the specific. For example we have

\section{"HEALTH EFFECTS CASES"}

as the general name and

\section{"ERL FAT/TOTAL $\quad 0-1609 \mathrm{KM}$}

as the specific part of the name. The consequence measure being presented on this line is the number of early fatality cases expected to occur in the region beginning at the facility ( 0 - $\mathrm{km}$ radius on our polar grid) and extending outward in a concentric ring to the spatial interval with an outer boundary at $1,609 \mathrm{~km}$.

Going across the page from left to right, there are ten columns of numeric data that provide a summary of the CCDF generated by the OUTPUT module. These are described as follows:

PROB NON-ZERO - Conditional probability of having a nonzero consequence estimate, conditional on the occurrence of the release under consideration. .

MEAN - $\quad$ - The average (expected) consequence over all weather trials. This is calculated by taking the sum of all the products [(consequence value) $\mathrm{X}$ (associated probability of that value)] for each weather trial.

50TH QUANTILE - The median of the estimated distribution function.

90TH QUANTULE - Based on the estimated distribution function, there is a $10 \%$ chance this consequence magnitude will be exceeded.

95TH QUANTILE - Based on the estimated distribution function, there is a $5 \%$ chance this consequence magnitude will be exceeded.

99TH QUANTLE - Based on the estimated distribution function, there is a $1 \%$ chance this consequence magnitude will be exceeded. 
99.9TH QUANTILE - Based on the estimated distribution function, there is a 0.1 $\%$ chance this consequence magnitude will be exceeded.

PEAK CONS

- The largest consequence magnitude obtained from the full set of weather trials which were examined.

PEAK PROB

- The probability associated with the largest consequence magnitude.

PEAK TRIAL - In the series of weather samples, the sequence number of the weather trial which gave rise to the largest consequence magnitude. By going back to the ATMOS output listing, the user can determine the start time (day and hour) of this weather sequence.

\section{C.12 User-Requested Cases of a Given Health Effect (T1)}

EARLY can calculate the number of health effect cases that will occur within a range of distances. The risk models for these health effects must have been previously defined in the proper section.

Variable Name - NUM1
Variable Type - Integer, Scalar
Allowed Range $-0<=$ value $<=40$
Explanation - The number of results of this type to be calculated.

.Variable Name - NAME

Variable Type - Character, Array

Allowed Range $-10<=$ length $<=24$

Explanation - Defines the name of each Type 1 result for which the number of cases will be calculated. Depending on the value of NAME, six different types of results may be produced. They are listed below along with examples of their specification for input.

1. cases of early fatality; 'ERL FAT/TOTAL'

2. cases of a given early injury; 'ERL INJ/name of an injury'

3. cases of a given cancer death; 'CANFAT/name of a cancer'

4. total cases of cancer death;

'CAN FAT/TOTAL'

5. cases of a given cancer injury; 'CAN INJ/name of a cancer'

6. total cases of cancer injury;

'CAN INJ/TOTAL'

Variable Name - I1DIS1

Variable Type - Integer, Array

Allowed Range $-1<=$ value $<=$ NUMRAD 
Explanation - Defines the inner spatial interval of the region of interest for this result. The user must supply NUM1 values in column two of the data block.

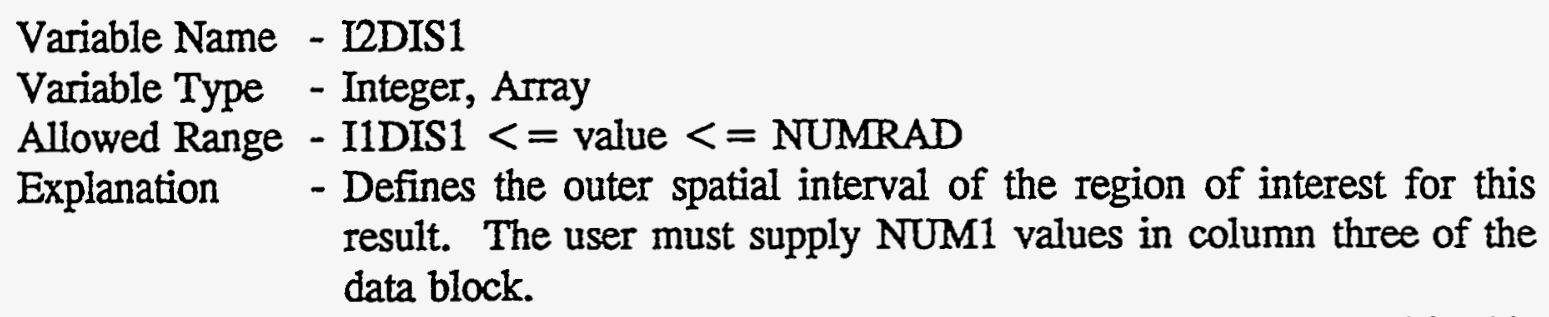

Note: In order to obtain the CCDF tables of a consequence measure requested in this section, append the character string 'CCDF' to the line requesting that result as the fourth item on the data card. The CCDF tables will be printed on the List Output File (unit 6).

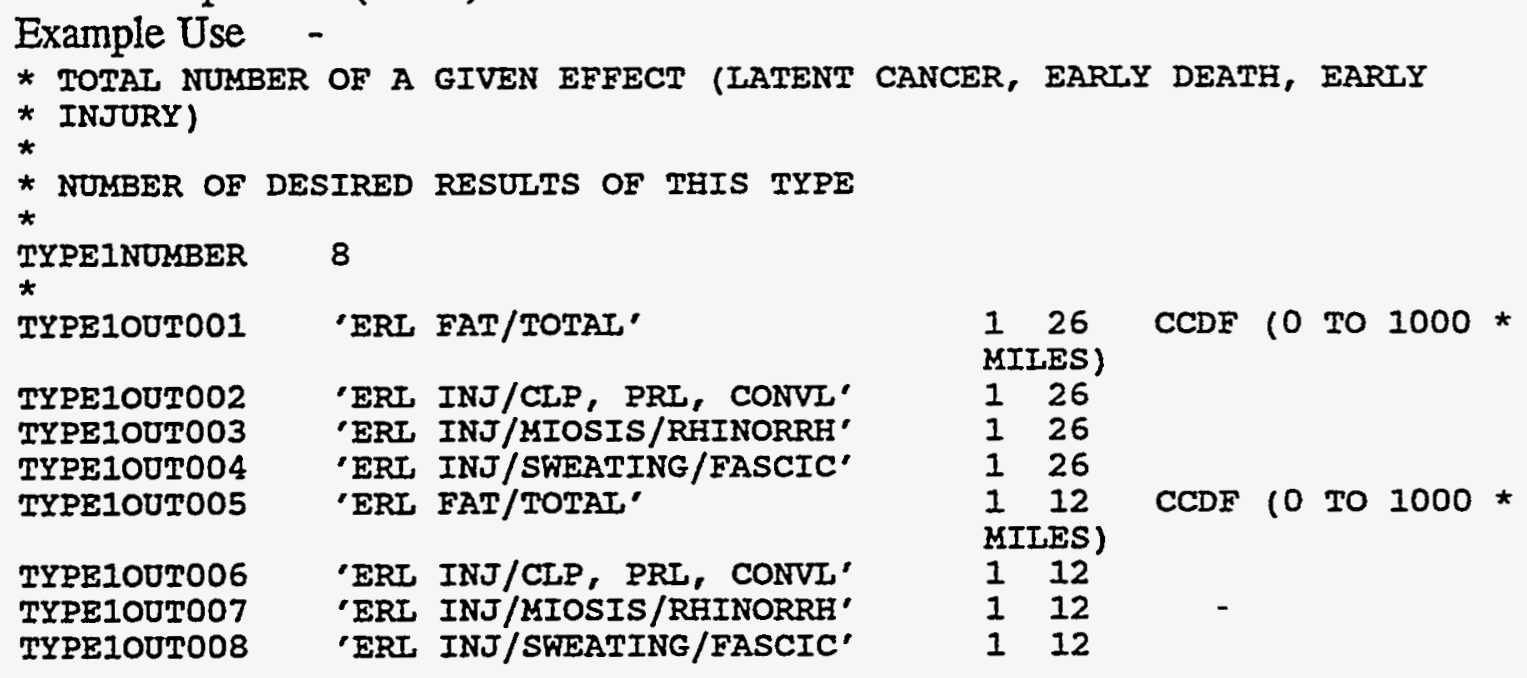

\section{C.13 User-Requested Early Fatality Radius (T2)}

It may be of interest to know the greatest distance at which a specified level of early fatality risk is exceeded. By turning on this result, the user can obtain information about the size of the region in which early fatalities are predicted to occur.

Variable Name - NUM2

Variable Type - Integer, Scalar

Allowed Range $-0<=$ value $<=10$

Explanation - The number of results of this type to be calculated.

Variable Name - RISTHR

Variable Type - Real, Scalar

Allowed Range $-0.0<=$ value $<=1.0$

Explanation - Defines the risk threshold used for calculating the fatality radius (reported in kilometers). The user must supply NUM2 values in column one of the data block. This is the only array in the data block. 
Note: In order to obtain the CCDF tables of a consequence measure requested in this section, append the character string 'CCDF' to the line requesting that result as the second item on the data card. The CCDF tables will be printed on the List Output File (unit 6).

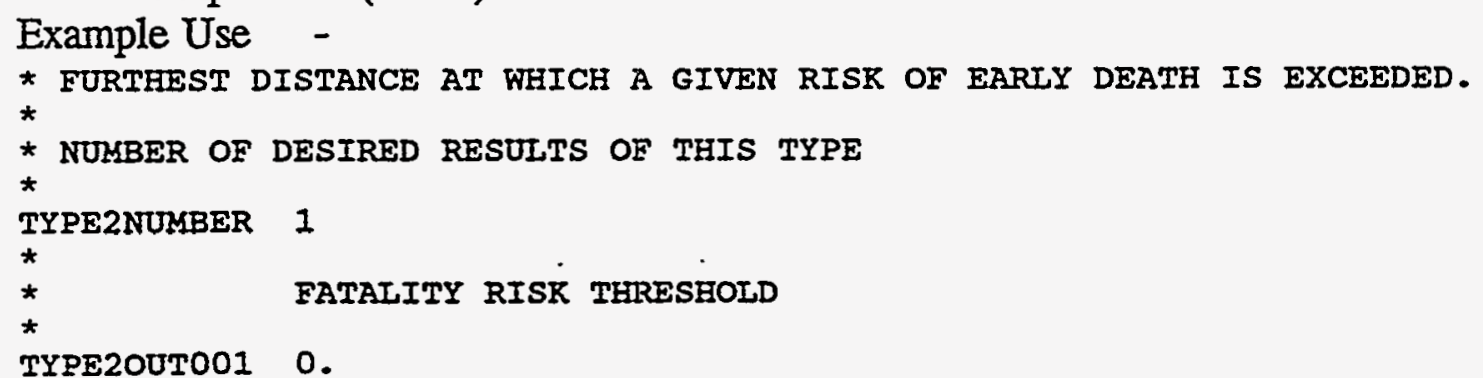

\title{
C.14 User-Requested Population Exceeding a Dose Threshold (T3)
}

Within the EARLY module alone, it may be of interest to know how many people received doses exceeding certain levels. This information can be obtained by requesting the production of the result described below. It is important to remember that this consequence measure is calculated solely on the basis of the dose calculations performed in the EARLY module.

The user can specify two types of doses to be used for the comparison: acute and lifetime. "Acute" dose is used by the code for calculating early fatality and early injury health effects (see Section C.8). For each organ, the "acute" dose is calculated with the set of dose reduction factors defined in that section. The lifetime dose is used for the calculation of cancer fatality and cancer injury health effects (see Section C.10). It represents the 0 - to 50-year lifetime dose commitment to a reference man which results from exposure during the emergency phase (EARLY) time period.

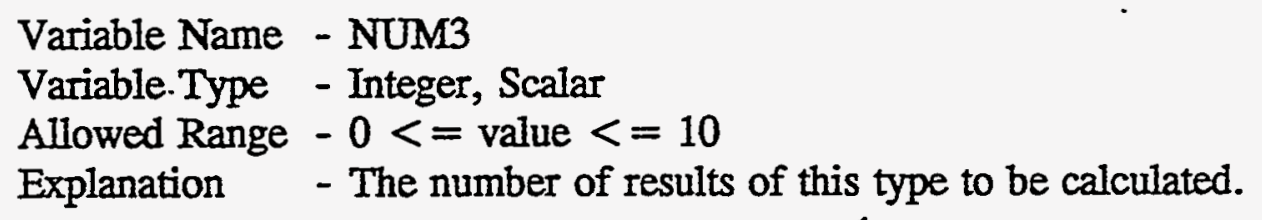

Variable Name - NUM3

Variable.Type - Integer, Scalar

Allowed Range $-0<=$ value $<=10$

Explanation - The number of results of this type to be calculated.

Variable Name - NAME

Variable Type - Character, Scalar

Allowed Range $-2<=$ length $<=10$

Explanation - Defines the name of the organ to which the dose threshold applies. This organ must be found on the list of organs, ORGNAM. The user must supply NUM3 values in column one of the data block.

\author{
Variable Name - DOSTH3 \\ Variable Type - Real, Array \\ Allowed Range $-0.0<=$ value $<=1000.0$
}


Explanation - Defines the dose threshold that will be used for counting the population. The user must supply NUM3 values in column two of the data block.

Note: In order to obtain the CCDF tables of a consequence measure requested in this section, append the character string 'CCDF' to the line requesting that result as the fourth item on the data card. The CCDF tables will be printed on the List Output File (unit 6).

Example Use

* NUMBER OF PEOPLE hHOSE DOSE TO a GIVEN ORGAN EXCEEDS a GIVEN THRESHOLD.

*

* NUMBER of desired results of this type

* TYPE3NUMBER 6

\begin{tabular}{|c|c|c|c|}
\hline & ORGAN NAME & DOSE THRES & HOLD \\
\hline PE3 & 'VIN1G & & \\
\hline E. & 'VSK & & \\
\hline PE3OUI & 'LSR & 15.0 & A \\
\hline YYPE3OUT004 & 'LSR4GA' & 8.8 & ACUTE \\
\hline PE3OUTO & 'VNE5GA' & 0.005 & ACUTE \\
\hline E3OUTO & 'VSK6GA' & 20.0 & ACUTE \\
\hline
\end{tabular}

\section{C.15 User-Requested Average Individual Risk (T4)}

Average individual risk is obtained by taking the sum of the risk values in all sectors at a given distance and dividing it by the number of sectors.

Note: Direct exposure includes the inhalation dose to the people living around the facility. The risk presented in this result does not include doses from ingestion of food and water by the region's population or doses to decontamination workers working in the region.

Variable Name - NUM4

Variable Type - Integer, Scalar

Allowed Range $-0<=$ value $<=20$

Explanation - The number of results of this, type to be calculated.

Variable Name - I1DIS4

Variable Type - Integer, Array

Allowed Range $-1<=$ value $<=$ NUMRAD

Explanation - Radial spatial interval of the distance of interest. The user must supply NUM4 values in column one of the data block.

Variable Name - NAME

Variable Type - Character, Array

Allowed Range $-10<=$ length $<=24$ 
Explanation - Name of the health effect risk. Average individual risk may be calculated for five types of health effects.

1. risk of early fatality; 'ERL FAT/TOTAL'

2. risk of a given early injury; 'ERL INJ/name of an injury'

3. risk of a given cancer death; 'CAN FAT/name of a cancer'

4. risk of cancer death; 'CAN FAT/TOTAL'

5. risk of a given cancer injury; 'CAN INJ/name of a cancer'

The user must supply NUM4 values of NAME in column two of the data block.

Note: In order to obtain the CCDF tables of a consequence measure requested in this section, append the character string 'CCDF' to the line requesting that result as the third item on the data card. The CCDF tables will be printed on the List Output File (unit 6).

Example Use -

* 360 DEGREE AVERAGE RISK OF A GIVEN EFFECT AT A GIVEN DISTANCE.

* POSSIBLE TYPES OF EFFECTS ARE:

* 'erL fat/total.

* 'ERL INJ/INJURY Name'

* 'CAN fat/CANcer nare'

* 'Can fat/total.

* NUMBER OF DESIRED RESULTS OF THIS TYPE

* TYPEANUMBER 6

*

$\star$

TYPE4OUTO01

TYPE40UT002

TYPE400T003

TYPE4OUTO04

TYPE4OUTOO5

TYPE4OUTO06

RADIAI INDEX

TYPE OF EFFECT

'ERL FAT/TOTAL'

'ERL FAT/TOTAL'

'ERI FAT/TOTAL'

'ERI FAT/TOTaI.

'ERI FAT/TOTaI"

'ERL FAT/TOTAL'

\section{C.16 User-Requested Population Dose (T5)}

The total long-term population dose to a given organ resulting from the contamination of a specified region can be calculated. The user must supply the name of the target organ as well as the inner and outer spatial intervals of the region of interest. If only the EARLY module is being run, this result reflects only the pathways considered by EARLY.

Variable Name - NUM5

Variable Type - Integer, Scalar

Allowed Range $-0<=$ value $<=10$

Explanation - The number of results of this type to be calculated.

Variable Name - NAME

Variable Type - Character, Scalar

Allowed Range $-2<=$ length $<=10$ 
Explanation - Defines the name of the organ to which the population dose applies. This organ must be found on the list of organs, ORGNAM. The user must supply NUMS values in column one of the data block.

Variable Name - I1DIS5

Variable Type - Integer, Array

Allowed Range $-1<=$ value $<=$ NUMRAD

Explanation - Defines the inner spatial interval of the region of interest for this result. The user must supply NUMS values in column two of the data block.

Variable Name - I2DIS5

Variable Type - Integer, Array

Allowed Range - IlDIS5 $<=$ value $<=$ NUMRAD

Explanation - Defines the outer spatial interval of the region of interest for this result. The user must supply NUM5 values in column three of the data block.

Note: In order to obtain the CCDF tables of a consequence measure re-quested in this section, append the character string 'CCDF' to the line requesting that result as the fourth item on the data card. The CCDF tables will be printed on the List Output File (unit 6).

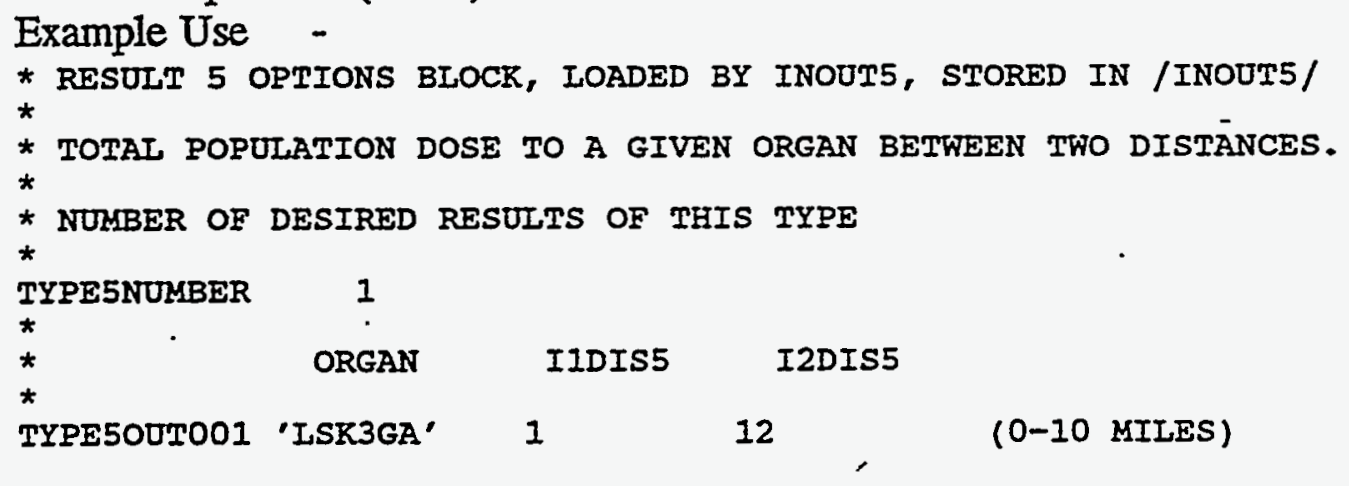

\section{C.17 User-Requested Centerline Dose vs. Distance (T6)}

If the straight-line plume model was chosen (IPLUME=1), the code can keep track of centerline dose between a range of distances for the various pathways. The centerline dose at each distance is treated as a separate result and OUTPUT will generate a set of results for each of the radial spatial intervals within the specified range. Depending on the exposure pathways specified, this result will be calculated by EARLY (see below). The pathway values are produced by EARLY. For a description of effective acute dose, please refer to Section C.8. 
An alternative method for examining centerline dose from EARLY alone is to set the output control variable, IPRINT, to a value greater than zero. If this is done, a listing of dose vs. distance for all the organs will be printed on the list output. Since this is written for each weather trial, it is recommended that this be done only for single weather trial runs.

$\begin{array}{ll}\text { Variable Name } & - \text { NUM6 } \\ \text { Variable Type } & - \text { Integer, Scalar } \\ \text { Allowed Range } & -0<=\text { value }<=10 \\ \text { Explanation } & - \text { The number of results of this type to be calculated. } \\ & \text { Note: unless IPLUME }=1 \text {, you must specify a value of zero. }\end{array}$

PATHNM DESCRIPTION

1. CLD cloudshine dose (not used in CHEM_MACCS)

2. GRD groundshine dose (not used in CHEM_MACCS),

3. INH ACU effective acute dose from inhalation of the plume as it passed overhead,

4. INH LIF lifetime dose commitment from inhalation of the plume as it passed overhead,

5. TOT ACU total effective acute dose being the sum of CLD, GRD, INH ACU, and RES ACU,

6. TOT LIF total lifetime dose commitment from all direct exposure pathways being the sum of CLD, GRD, INH LIF, and RES LIF,

7. RES LIF lifetime dose commitment from inhalation of resuspended material after plume passage,

8. RES ACU effective acute dose from inhalation of resuspended material after plume passage.

Note: The pathway name for 'SKIN' will ignored by the code since there is only one exposure pathway for skin (direct dry deposition to the skin). The user must supply a value for the pathway name in all cases. For the case of skin as the target organ, it does not matter which of the eight pathways listed above is supplied.

Variable Name - ORGNAM

Variable Type - Character, Scalar

Allowed Range $-2<=$ length $<=10$

Explanation - Defines the names of the organs for which centerline doses are to be reported. These organs must be found on the list of organs, ORGNAM. The user must supply NUM6 values in column one of the data block. 
Variable Name - PATHNM

Variable Type - Character, Scalar

Allowed Range $-3<=$ length $<=7$

Explanation - Defines the names of the pathways for which centerline doses are to be reported. The name of each pathway must be on the list of pathways below. The user must supply NUM6 values in column two of the data block.

Variable Name - I1DIS6

Variable Type - Integer, Array

Allowed Range $-1<=$ value $<=$ NUMRAD

Explanation - Defines the inner spatial interval of the region of interest for this result. The user must supply NUM6 values in column three of the data block.

Variable Name - I2DIS6

Variable Type - Integer, Array

Allowed Range - I1DIS6 $<=$ value $<=$ NUMRAD

Explanation - Defines the outer spatial interval of the region of interest for this result. The user must supply NUM6 values in column four of the data block.

Note: In order to obtain the CCDF tables of a consequence measure requested in this section, append the character string 'CCDF' to the line requesting that result as the fifth item on the data card. The CCDF tables will be printed on the List Output File (unit 6).

Example Use -

* CENTERLINE DOSE TO AN ORGAN VS DIST BY PATHWAY, PATHWAY NAMES ARE AS

* FOLIOWS:

* PATHWAY NAME:

* 'CLD' - CLOUdSEINE (not used in CHEM MACCS)

* 'GRD' - GROUNDSEINE (not used in CFIFT MACCS)

* 'INH ACU'-"ACUTE DOSE EQUIVALENT" FROM DIRECT INHALATION OF THE

$\star$

'INH LIF'-"IIFETIME DOSE COMMITMENT" FROM DIRECT INHALATION OF THE CLOUD

'RES ACU'-"ACUTE DOSE EQUIVALENT" FROM RESUSPENSION INHALATION

'RES IIF'-"IIFETIME DOSE COMMITMENT" FROM RESUSPENSION INHALATION

'TOT ACU'-"ACUTE DOSE EQUIVALENT" FROM AIL PATHWAYS

'TOT IIF'-"IIFETIME DOSE COMMITMENT" FROM ALI PATHWAYS

* NOMBER OF DESIRED RESULTS of ThIS TyPE

*

TYPE6NUMBER 1

*

$\star$

TYPE600T001 'VINIGA' 'TOT ACU'

*TYPE6OUTO02 'ISR3GA'

'TOT IIF'

IIDIS6

I2DIS6

$1 \quad 19$ (0-50 MILES)

126 (0-1000 MILES) 


\section{C.18 User-Requested Centerline Risk vs. Distance (T7)}

If the straight-line plume model was chosen (IPLUME=1), the code can keep track of centerline risk between two specified spatial intervals for the various types of health effects. Centerline risk is calculated for hypothetical individuals located directly under the path of the plume who are exposed to the Gaussian peak of the air and ground concentrations. The centerline risk at each distance in the region is treated as a separate result and OUTPUT will generate a distribution of the consequence measure for each of the spatial intervals within the specified range.

Note: The risk presented in this result does not include societal doses from ingestion of any food and water contaminated as a result of the accident or doses to decontamination workers working in the contaminated area.

Variable Name - NUM7

Variable Type - Integer, Scalar

Allowed Range $-0<=$ value $<=10$

Explanation - The number of results of this type to be calculated.

Note: Unless IPLUME=1, you must specify a value of zero.

Variable Name - NAME

Variable Type - Character, Array

Allowed Range $-10<=$ length $<=24$

Explanation - Defines the option for results of Type 7, centerline risk of a given type of health effect. Depending on the value of NAME, six different types of results may be produced. They are listed below along with examples of their use. The user must supply NUM7 values in column one of the data block.

1. risk of early fatality; 'ERL FAT/TOTAI'

2. risk of a given early injury; 'ERL INJ/name of an injury'

3. risk of a given cancer death; 'CAN FAT/name of a cancer'

4. risk of cancer death; 'CAN FAT/TOTAL'

5. risk of a given cancer injuŕry; 'CAN INJ/name of a cancer'

6. risk of cancer injury; 'CAN INJ/TOTAL' 
Variable Name - IIDIS7

Variable Type - Integer, Array

Allowed Range $-1<=$ value $<=$ NUMRAD

Explanation - Defines the inner spatial interval of the region of interest for this result. The user must supply NUM7 values in column two of the data block.

Variable Name - I2DIS7

Variable Type - Integer, Array

Allowed Range - IIDIS7 < = value $<=$ NUMRAD

Explanation - Defines the outer spatial interval of the region of interest for this result. The user must supply NUM7 values in column three of the data block.

Note: In order to obtain the CCDF tables of a consequence measure requested in this section, append the character string 'CCDF' to the line requesting that result as the fourth item on the data card. The CCDF tables will be printed on the List Output File (unit 6).

\section{Example Use -}

* CENTERLINE RISR OF A GIVEN EFFECT VS DISTANCE

* NuMber of DESIRED REsUlts of this type

*

TYPE7NUMBER 1

$\star$

TYPE7OUTOOI

*TYPE7OOTOO2

$\begin{array}{ccc}\text { NAME } & \text { IIDIS7 } & \text { I2DIS7 } \\ \text { 'ERT FAT/TOTAI' } & 1 & 19 \\ \text { 'CAN FAT/TOTAL' } & 1 & 26\end{array}$

(0-50 MILES)

(0-1000 MILES)

\section{C.19 User-Requested Population-Weighted Risk (T8)}

The population-weighted health effect risk is obtained by calculating the cases of a health effect in a certain region and then dividing by the total population in the region. It takes account of both the population distribution and the wind rose.

Note: The risk presented in this result does not include the societal pathways of (1) ingestion of contaminated food and water'or (2) doses to decontamination workers working in the area.

Variable Name - NUM8

Variable Type - Integer, Scalar

Allowed Range $-0<=$ value $<=20$

Explanation - The number of results of this type to be calculated.

Variable Name - NAME

Variable Type - Character, Array

Allowed Range $-10<=$ length $<=24$

Explanation - Defines the names of the Type 8 results to be calculated: populationweighted risk of a given type health effect. Depending on the value 
of NAME, six different types of results may be produced. These six options are listed below along with examples of their use. The user must supply NUM8 values in column one of the data block.

1. risk of early fatality; 'ERL FAT/TOTAL'

2. risk of a given early injury; 'ERL INJ/name of an injury'

3. risk of a given cancer death; 'CAN FAT/name of a cancer'

4. risk of cancer death; 'CAN FAT/TOTAL'.

5. risk of a given cancer injury; 'CAN INJ/name of a cancer'

6. risk of cancer injury; 'CAN INJ/TOTAL'

Variable Name - IIDIS8

Variable Type - Integer, Array

Allowed Range $-1<=$ value $<=$ NUMRAD

Explanation - Defines the inner spatial interval of the region of interest for this result. The user must supply NUM8 values in column two of the data block.

Variable Name - I2DIS8

Variable Type - Integer, Array

Allowed Range $-1<=$ value $<=$ NUMRAD

Explanation - Defines the outer spatial interval of the region of interest for this result. The user must supply NUM8 values in column three of the data block.

Note: In order to obtain the CCDF tables of a consequence measure requested in this section, append the character string 'CCDF' to the line requesting that result as the fourth item on the data card. The CCDF tables will be printed on the List Output File (unit 6).

Example Use -

* POPULATION WEIGHTED FATALITY RISK BETWEEN 2 DISTANCES

* NOMBER OF DESIRED RESULTS OF this tyPE

*

TYPE8NUMBER 1

*

* NAME IIDIS8 I2DIS8

TYPE8OUTOO1 'ERT FAT/TOTAL' 1 TYPEOUTO02 'CAN

*TYPE8OUTOO2 'CAN FAT/TOTAL' 1 12 CCDF (0-10 MIIES)

\section{C.20 User-Requested Early Fatality Radius (T9)}

It may be of interest to know the greatest distance at which a specified level of early injury risk is exceeded. By turning on this result, the user can obtain information about the size of the region in which an early injury is predicted to occur. 
Variable Name - NUM9

Variable Type - Integer, Scalar

Allowed Range $-0<=$ value $<=10$

Explanation - The number of results of this type to be calculated.

Variable Name - RISTHR

Variable Type - Real, Scalar

Allowed Range $-0.0<=$ value $<=1.0$

Explanation - Defines the risk threshold used for calculating the injury radius (reported in kilometers). The user must supply NUM9 values in column one of the data block. This is the only array in the data block.

Note to Users: In order to obtain the CCDF tables of a consequence measure requested in this section, append the character string 'CCDF' to the line requesting that result as the second item on the data card. The CCDF tables will be printed on the List Output File (unit 6).

Example Use of NUM9 and RISTHR:

* FURThest DISTANCE AT WHICh a GIVEN RISK OF EARLY INJURY IS EXCEEDED.

*

* NOMBER OF DESIREd results of this type

*

TYPE9NUMBER 3

*

*

$\star$

*

*

TYPE9OUTO01

TYPE9OUT002

TYPE9OUTO03

INJURY RISR THRESHOLD

$\begin{array}{ccc}\text { EINAME } & \begin{array}{c}\text { RISK } \\ \text { THRESHOLD }\end{array} & \begin{array}{l}\text { DOSE } \\ \text { FLAG }\end{array} \\ \text { 'CLP, PRL, CONVL' } & 0.1 & \\ \text { 'MIOSIS/RHINORRH' } & 0.1 & \text { ACUTE } \\ \text { 'SWEATING/FASCIC' } & 0.1 & \text { ACUTE }\end{array}$

\section{C.21 User-Requesfed Population Exceeding a Dose Threshold (T10)}

It may be of interest to know the area of land contaminated in excess of a certain level. This information can be obtained by requésting results as described below. This consequence measure is obtained on the basis of the ground contamination calculations performed in the EARLY module.

Variable Name - NUM0

Variable Type - Integer, Scalar

Allowed Range $-0<=$ value $<=10$

Explanation - The number of results of this type to be calculated.

Variable Name - NAMEO

Variable Type - Character, Scalar

Allowed Range $-2<=$ length $<=10$ 
Explanation - Defines the name of the chemical species for which the land contamination threshold applies. This chemical species must be found on the list, NUCNAM. The user must supply NUMO values in column one of the data block.

Variable Name - GCTHRO

Variable Type - Real, Array

Allowed Range $-0.0<=$ value $<=1000.0$

Explanation

- Defines the ground concentration $\left(\mathrm{kg} / \mathrm{m}^{2}\right)$ threshold that will be used. The user must supply NUM0 values in column two of the data block.

Note to Users: In order to obtain the CCDF tables of a consequence measure requested in this section, append the character string 'CCDF' to the line requesting that result as the fourth item on the data card. The CCDF tables will be printed on the List Output File (unit 6).

Example Use of NUM0, NAME0, and GCTHR0

* AREA WITH GROUND CONCENTRATION OF A GIVEN AGENT EXCEEDING A THRESHOLD

*

* NUMBER OE DESIRED RESULTS OF THIS TYPE

*

TYPEONUNBER 4

*

*

*

*

TYPEOOUT001

TYPEOOUT002

TYPEOOUTO03

TYPEOOUTO04

SPECIES

NAME

'GA-IIQ'

'GB-IIQ'

'VX-IIQ'

'ED-IIQ'
GROUND CONC.

THRESHOLD DOSE

$\left(\mathrm{kg} / \mathrm{m}^{\wedge} 2\right)$ FLAG

$0.1 E-3$ ACUTE

$0.1 \mathrm{E}-3$ ACUTE

$0.1 E-3$ ACUIE

$0.1 E-3$ ACUTE 


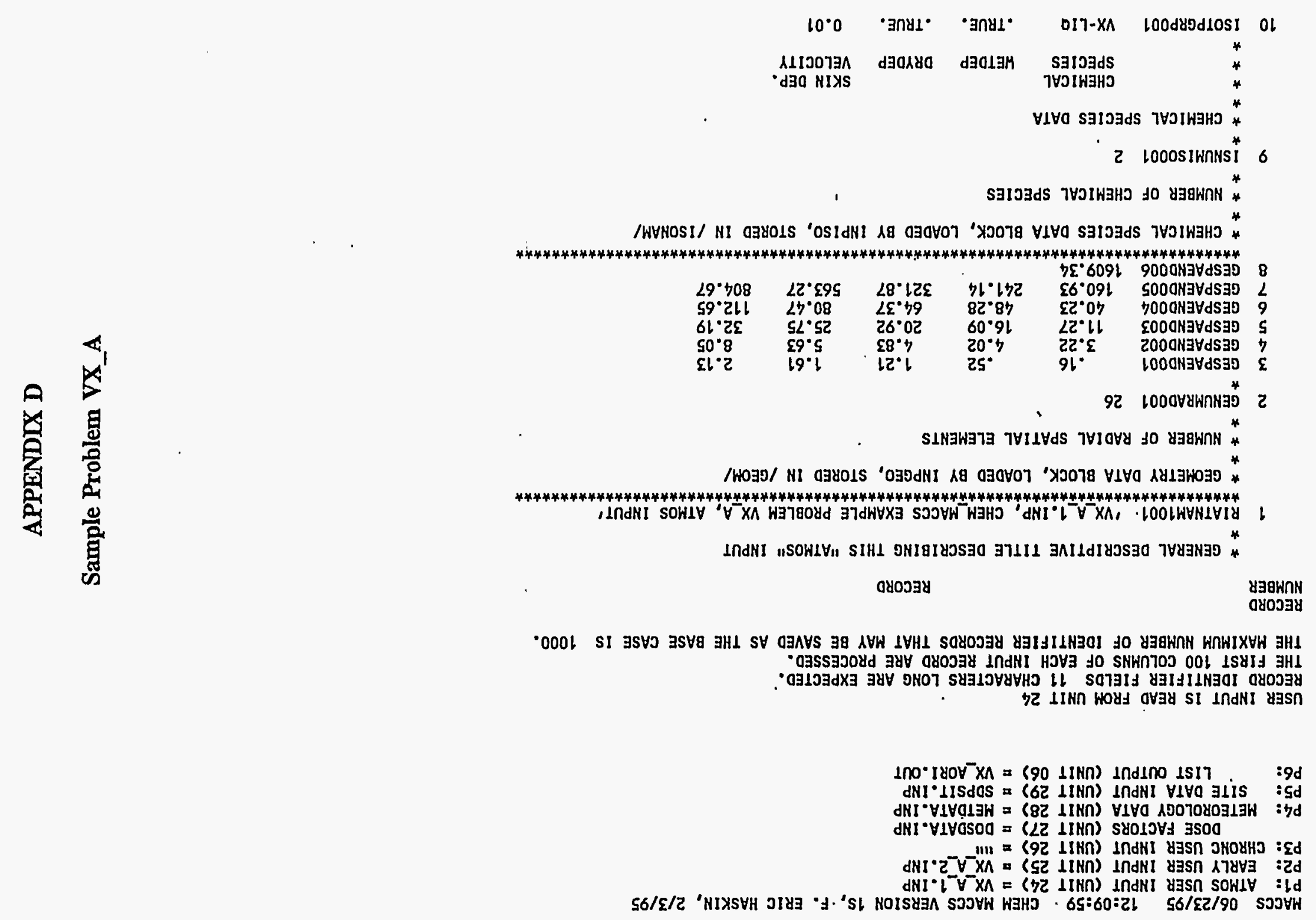


11 I SOTPGRP002 VX-VAP .TRUE. .TRUE. 0.01

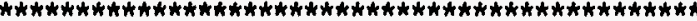
* HET DEPOSITION DATA BLOCK, LOADED BY INPHET, STORED IN /HETCON/

* hashout coefficient number one, lihear factor $\star$

12 HDCHASH1001 9.5E-5 (JON HELTON AFTER JONES, 1986) * HASHOUT COEFFICIENT MUMBER TWO, EXPONENTIAL. FACTOR *

13 HDCHASH2001 0.8 (JON HELTON AFTER JONES, 1986)

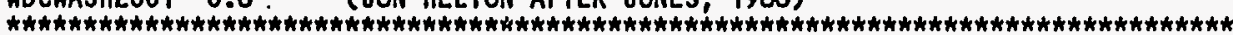
* DRY DEPOSITION DATA BLOCK, LOADED BY INPDRY, STORED IN /DRYCON/ * number of particle size groups

14 DDNPSGRPOO1

* deposition Velocity of Each particle size group (h/S)

15 DDVDEPOSO01 $0.001 \quad 0.01$

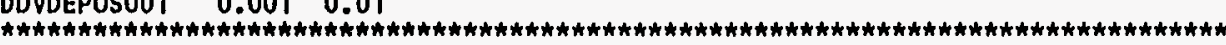
* DISPERSION PARAMETER DATA BLOCK, LOADED bY INPDIS, STORED IN /DISPY/, IDISPZ/

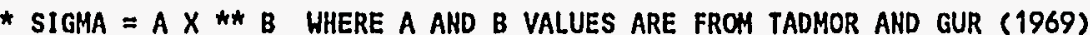

* LINear term of the expression for sigma-y, 6 stabilltiy classes * stabillitr class: A B C D

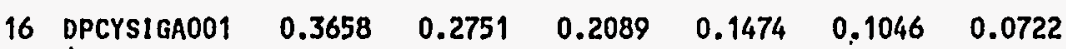
* EXPONENTIAL TERM OF THE EXPRESSION FOR SIGMA-Y, 6 STABILITY CLASSES *

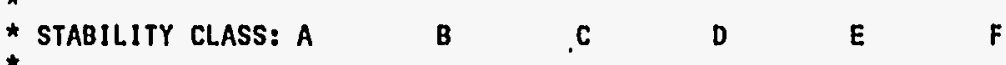

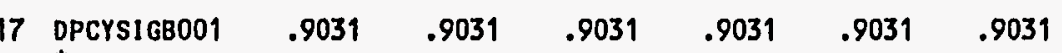
* linear term of the expression for sigma-z, 6 stability classes

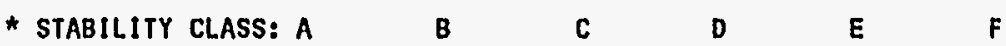

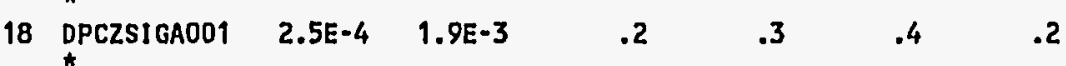
* EXPONENTIAL TERM OF THE EXPRESSION FOR SIGMA-2, 6 STABILITY CLASSES * STA 


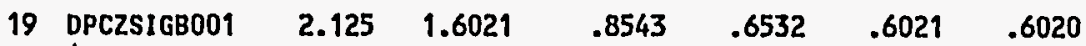

* Limear scaling factor for sigma-y function, normally 1

20

DPYSCALEOOI 1.

* linear scaling factor for sigma-z function,

* MORHALly USED fOR SURFACE ROUGHNESS LENGTH CORRECTION.

* $(21 / \mathrm{ZO})$ ** 0.2, FROH CRAC2 HE HAVE ( $10 \mathrm{CH} / 3 \mathrm{CH}) * * 0.2=1.27$

21 DPZSCALEOO1 1.27

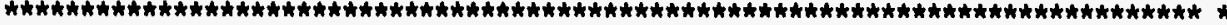

* EXPANSION FACTOR DATA BLOCK, LOADED BY INPEXP, STORED IN /EXPAND/

* tIME base for EXPANSION factor (SECONDS)

22 PMTIMBASO01 600. (10 MINUTES)

* bReak point for formula change (SECONDS)

23 PMBRKPNT001 3600. (1 HOUR)

* exponential expansion factor number 1

24 PMXPFAC1001 0.2

* EXPONENTIal eXPansion factor nUMBer 2

PMXPFAC2001

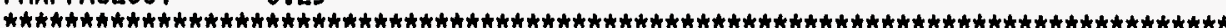

$\star$ PLUME RISE DATA BLOCK, LOADED BY INPLRS, STORED IN /PLUMR/

* scaling factor for the critical hind speed for entrainment of a bouyant plume

* (USED BY FUNCTION CAUGHT)

26 PRSCLCRHO01 1.

* scaling factor for the a-d stabilltty plume rise formula

* (USEO BY FUNCTION PLMRIS)

27 PRSCLADPOOI 1.

* Scaling factor for the e-F stabillity plume rise formula

* (USED BY FUNCTION PLMRIS)

28 PRSCLEFP001 1

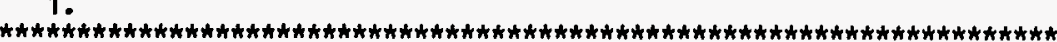

* haKe EFFECTS DATA BLOCK, LOADED BY INPHAK, STORED IN /BILHAK/ 


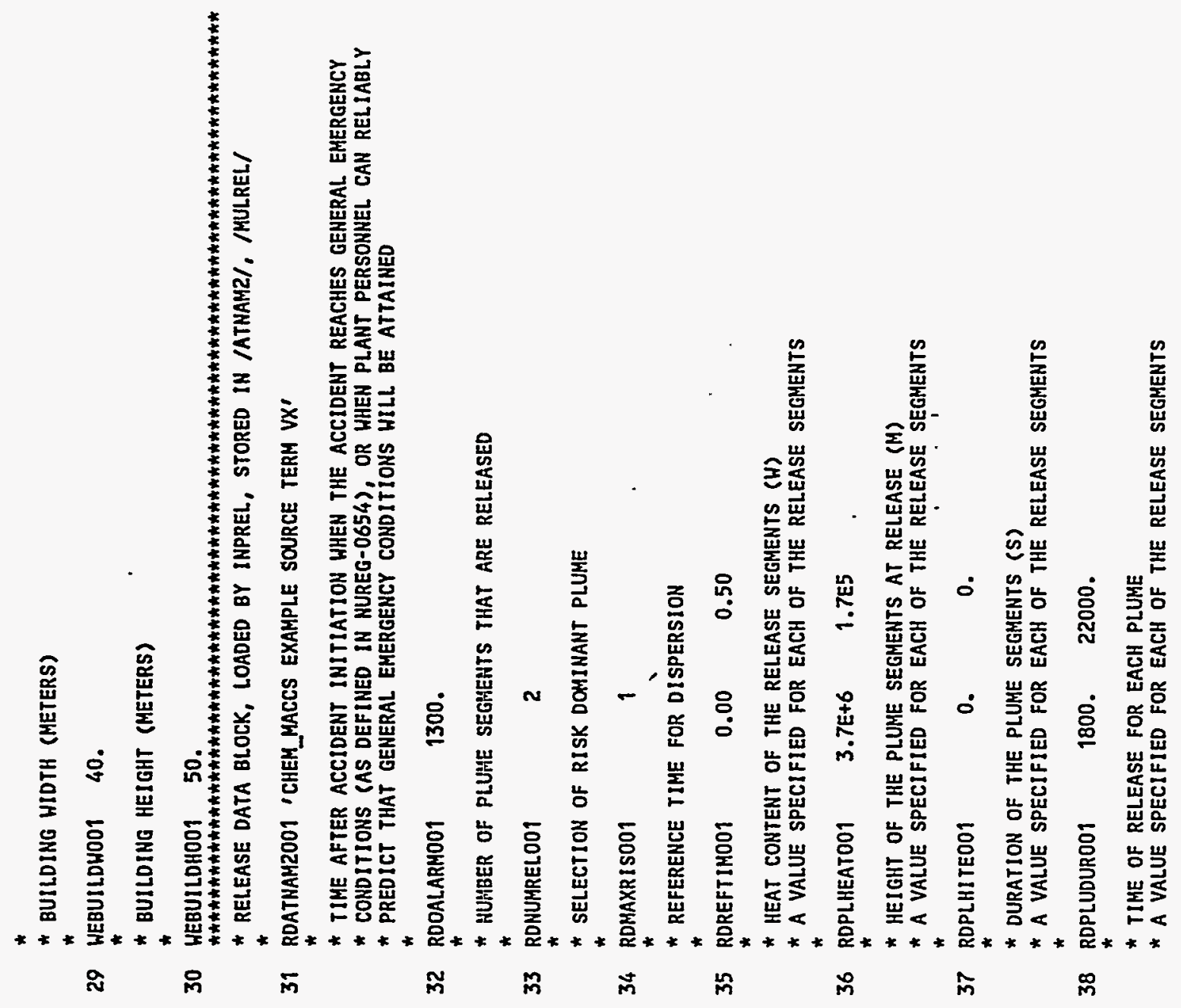


RDPDELAYO01 3700.10000.

* particle size distribution of each chemical species

* THE FRACTIONS FOR EACH CHEMICALL SPECIES (ROW) MUST SUH TO ONE.

*

$0.001 \mathrm{~m} / \mathrm{s} \quad 0.01 \mathrm{~m} / \mathrm{s}$

$\begin{array}{lllll}40 & \text { RDPSDIST001 } & 0.0 & 1.0 & \text { *VX-LIQ } \\ 41 & \text { RDPSDIST002 } & 1.0 & 0.0 & \text { *VX-VAP }\end{array}$

RDSDIST002

* InVEntory of each chemical species subJect to Release

chemical. Quantity

RDCORINVO01 VX-LIQ 1.000E+02

43 RDCORIHVOO2 VX-VAP $1.000 E+02$

* Scaling factor to adJust the inventory

*

44

ROCORSCAOOT 1.000

* release fractions for chemical species by plume

* PLUME: VX-LIa VX-VAP

5 RDRELFRCO01 $0.9 E+0 \quad 0.9 E+0$

46 RDRELFRCO02 $0.1 E+0 \quad 0.1 E+0$

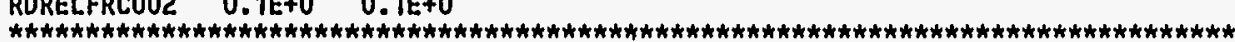

* OUTPUT CONTROL DATA BLOCK, LOADED BY INPOPT, STORED IN /STOPME/, /ATMOPT/

* flag to indicate that this is the last program in the series to be run

4 OCENDAT1001 .FALSE. (SET thIS VALUE TO .TRUE. TO SKIP EARLY AND CHRONC)

48 OCIDEBUGOOT 0

* hame of the chenical species to be listed on the dispersion listings

*OCNUCOUTO01 VX-VAP

等

* METEOROLOGICAL SAMPLIHG DATA BLOCK

* meteorological sampling option code: 
* MetCOD = 1, USER SPECIFIEd day and hOUR IN the year (From Met file), 2, HEATHER CATEGORY BIN SAMPLING

3, 120 HOURS OF WEATHER SPECIFIED ON THE ATMOS USER INPUT FILE,

4, CONSTANT MET (BOUHDARY HEATHER USED FROH THE START),

5, STRATIFIED RANDOH SAMPLES FOR EACH DAY OF THE YEAR.

49 H1HETCODO01 2

* hOUR of DAY InTERVAl. IH WHICH ACCIDENT MUST BEgIN

*

50 M1HRINITOO

HRINIA HRINIB

* last spatial interval for measured heather

51 MLLIMSPAOO1 25

* BoUndary heather mIXING LAYER heIGHT

$$
\text { * }
$$

52 M2BNDMXHOO1 1000. (METERS) * boundary heather stability class indeX

53 M2IBDSTBOOI 4 (D-STABILITY)

* boUndary heather Rain. RATE

* boUndary heather rain Rate

54 MZBNDRANOO1 5 . (MM/HR)

* boundar y heather hind speed

55 M2BNDWNDO01 $5 . \quad(M / S)$

* number of rain distance intervals for binning

56 MANRHINTOO1 5

* ENDPOINTS OF THE RAIN DISTANCE INTERVALS (KILOMETERS)

* NOTE. THese must ge chosen to match the spatial endpoint distances

* SPECIFIED FOR THE ARRAY SPAEND (10\% ERROR IS ALLOHED).

57 MLRNDSTS001 $3.22 \quad 5.63 \quad 11.27 \quad 20.92 \quad 32.19$

* number of rain INTENSITIY breakpoints

58 MANRINTNOO1 3 
* RAIN INTENSITY BREAKPOINTS FOR HEATHER BINNING (HILLLIMETERS PER HOUR)$$
\text { * }
$$

59 M4RHRATE001 2. 4. 6.

* nUMBer of samples PER bih

60 MANSMPLSOO1 4 (THIS NUMBER SHOULD BE SET TO 4 FOR RISK ASSESSHENT)

* INItIal SEed for Rahdom number generator

61 MLIRSEEDOO1 79

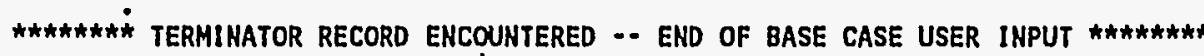

USER INPUT PROCESSING SUMHARY - BASE CASE

NUMBER OF RECORDS READ

NUMBER OF BLANK OR COMMENT RECORDS READ

NUMBER OF TERHINATOR RECOROS

NUMBER OF RECORDS PROCESSED

NUMBER OF PROCESSED RECORDS DUPLICATED

NUMBER OF PROCESSED RECORDS SORTED

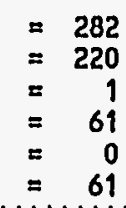

WER OF PROCESSED RECORDS SORTED

1 RELEASED INVENTORY OF ALL PLUMES
VX-LIQ
VX-VAP

VX-VAP $\quad 9.00 E+01 \quad 1.00 E+01$

READING FROH A WEATHER FILE WITH THE FOLLOHING HEADER:

CHEM_MACCS MET DATA (SURRY, NRC-12/12/88, CREATED 12/22/88)

MACCS FORMAT - NUREG-1150

METEOROLOGICAL DATA FILE CONTAINS 451 HOURS OF OBSERVED RAIN DATA.

ACCUMULATED RAIN MEASURENENTS TOTALED 29.21 INCHES FOR THE YEAR.

CONSTANT LID HEIGHTS (N) FOR 4 SEASONS $=\begin{array}{llll}1054 & 1890 & 1924 & 1412\end{array}$

0 NON-ZERO HINDSPEEDS LESS THAN $0.5 \mathrm{H} / \mathrm{S}$ ARE SET TO $0.5 \mathrm{M} / \mathrm{S}$

\section{BIN PRIORITIES}

**** MEteorologícal bin summary *** *

RI XX - RAIN INTENSITY I HITHIN THE INTERVAL ENDING AT XX

INTERVAL ENDPOINTS ARE IN KILOHETERS FROM THE ACCIDENT SITE, THE 5 INTERVAL ENDPOIHTS ARE

$S \quad V$ - INITIAL HEATHER CONDITIONS HITH STABILITY CLASS S AND HIND SPEED INTERVAL $V$

STABILITY CLASSES ARE $B=A / B, D=C / D, E=E$, AND $F=F$

HIND SPEED INTERVALS ARE IN METERS PER SECOND, $1(0-1), 2(1-2), 3(2-3), 4(3-5), 5(5-7), 6$ (GT 7 ) 
$\begin{array}{lllllllllllllllllllllll}1 \text { B } 3 & 0.032 & 0.041 & 0.037 & 0.029 & 0.077 & 0.088 & 0.080 & 0.055 & 0.060 & 0.046 & 0.039 & 0.069 & 0.089 & 0.090 & 0.130 & 0.039 & 957\end{array}$

2 B 4 4 $0.008 \quad 0.075 \quad 0.122 \quad 0.0800 .0420 .093 \quad 0.088 \quad 0.094 \quad 0.094 \quad 0.067 \quad 0.053 \quad 0.091 \quad 0.066 \quad 0.0110 .013 \quad 0.004 \quad 946$

$\begin{array}{llllllllllllllllllllllll}3 & 1 & 0.058 & 0.093 & 0.047 & 0.047 & 0.116 & 0.047 & 0.023 & 0.058 & 0.081 & 0.047 & 0.047 & 0.035 & 0.047 & 0.070 & 0.128 & 0.058 & 86\end{array}$

4 D 2. $0.0350 .040 \quad 0.054 \quad 0.0630 .052 \quad 0.080 \quad 0.052 \quad 0.072 \quad 0.070 \quad 0.072 \quad 0.075 \quad 0.0590 .0840 .096 \quad 0.0520 .042572$

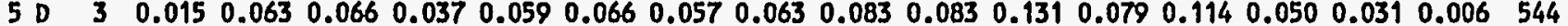

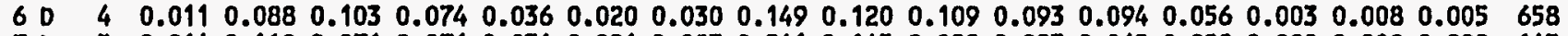

$\begin{array}{lllllllllllllllllllll}7 \mathrm{D} & 5 & 0.014 & 0.110 & 0.076 & 0.076 & 0.034 & 0.021 & 0.083 & 0.241 & 0.145 & 0.028 & 0.083 & 0.062 & 0.028 & 0.000 & 0.000 & 0.000 & 145\end{array}$

$8 \mathrm{D} \quad 6 \quad 0.000 \quad 0.3640 .000 \quad 0.000 \quad 0.0910 .000 \quad 0.000 \quad 0.000 \quad 0.000 \quad 0.000 \quad 0.000 \quad 0.545 \cdot 0.000 \quad 0.0000 .0000 .000 \quad 11$

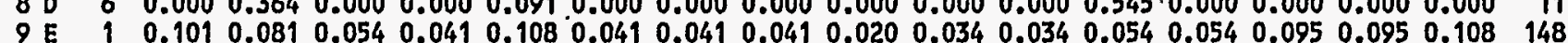

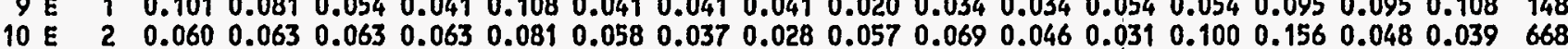

$\begin{array}{lllllllllllllllllllllll}11 & 3 & 0.063 & 0.135 & 0.080 & 0.057 & 0.036 & 0.036 & 0.040 & 0.078 & 0.135 & 0.077 & 0.090 & 0.018 & 0.063 & 0.053 & 0.015 & 0.026 & 758\end{array}$

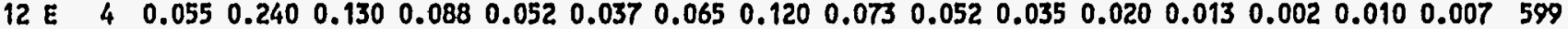

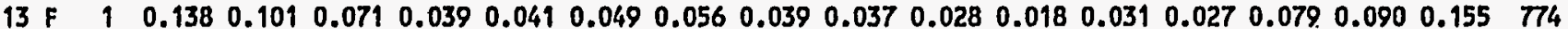

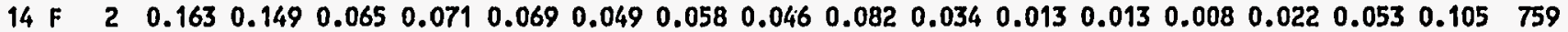

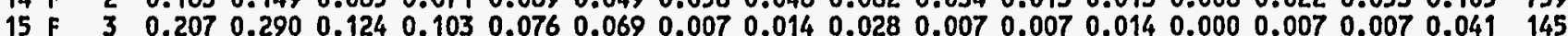

$16 \mathrm{~F} \quad 4 \quad 0.1670 .3330 .167 \quad 0.000 \quad 0.0830 .000 \quad 0.000 \quad 0.1670 .0830 .000 \quad 0.0000 .0000 .000 \quad 0.000 \quad 0.000 \quad 0.000 \quad 12$

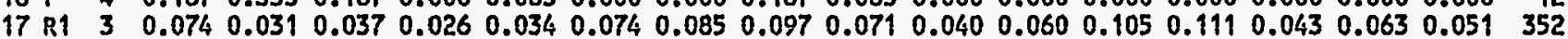

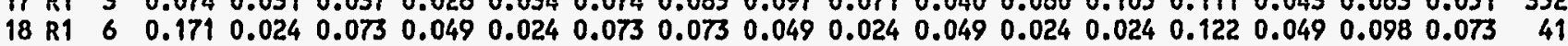

19 R1 $110.0970 .0490 .0490 .0100 .0100 .0580 .0390 .0390 .0490 .0290 .0870 .0680 .165 \quad 0.0100 .097 \quad 0.146 \quad 103$

20 R1 $21 \quad 0.098 \quad 0.076 \quad 0.0450 .015 \quad 0.008 \quad 0.053 \quad 0.068 \quad 0.053 \quad 0.030 \quad 0.038 \quad 0.045 \quad 0.098 \quad 0.144 \quad 0.0380 .106 \quad 0.083 \quad 132$

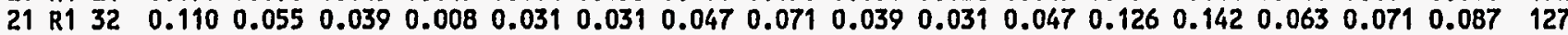

$222_{3} \quad 0.1080 .0410 .0270 .0680 .0410 .0000 .0140 .0680 .0680 .0680 .1080 .1350 .0810 .0810 .0680 .027$

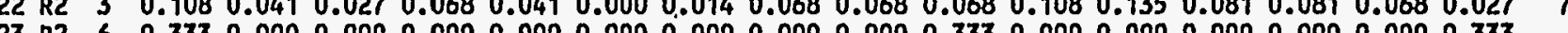

24 R2 $11 \quad 0.125 \quad 0.000 \quad 0.125 \quad 0.000 \quad 0.000 \quad 0.000 \quad 0.000 \quad 0.063 \quad 0.125 \quad 0.063 \quad 0.063 \quad 0.000 \quad 0.063 \quad 0.063 \quad 0.250 \quad 0.063$

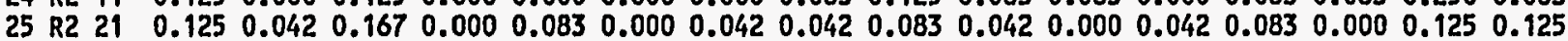

26 R2 $320.148 \quad 0.000 \quad 0.074 \quad 0.0370 .0740 .037 \quad 0.000 \quad 0.000 \quad 0.000 \quad 0.000 \quad 0.0370 .0370 .1850 .0370 .0740 .259$

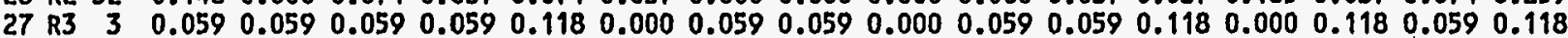

$29 \mathrm{R3} 11 \quad 0.333 \quad 0.000 \quad 0.000 \quad 0.000 \quad 0.000 \quad 0.000 \quad 0.000 \quad 0.000 \quad 0.000 \quad 0.000 \quad 0.000 \quad 0.000 \quad 0.000 \quad 0.333 \quad 0.0000 .333$

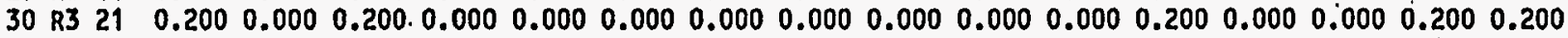

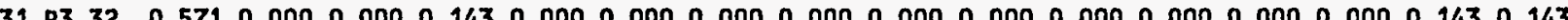

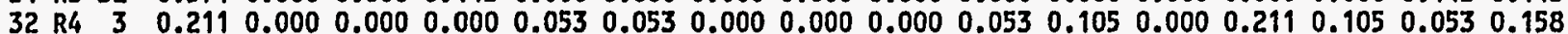

33 R4 6 6 $0.0000 .000 \quad 0.000 \quad 0.000 \quad 0.000 \quad 0.000 \quad 0.000 \quad 0.000 \quad 0.000 \quad 0.000 \quad 0.000 \quad 0.0000 .0000 .0000 .0001 .000$

34 R4 $11 \quad 0.000 \quad 0.000 \quad 0.000 \quad 0.000 \quad 0.000 \quad 0.000 \quad 0.000 \quad 0.000 \quad 0.000 \quad 0.000 \quad 0.000 \quad 0.0000 .400 \quad 0.0000 .200 \quad 0.400$

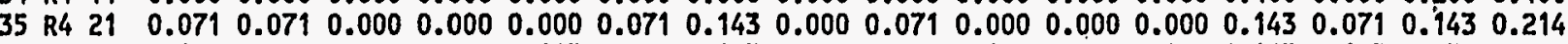

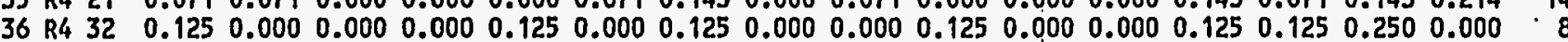

$37 \mathrm{ALL}$

$\begin{array}{lllllllllllllllllllllllll}0.066 & 0.095 & 0.075 & 0.056 & 0.054 & 0.057 & 0.057 & 0.074 & 0.077 & 0.057 & 0.055 & 0.056 & 0.066 & 0.054 & 0.052 & 0.050 & 8760\end{array}$

PER CEHT

BIN PRIORITIES

$$
\text { * * * * METEOROLOGICAL BIN SUMMARY * * * * }
$$

RI XX - RAIN INTENSITY I HITHIN THE INTERVAL ENDIHG AT XX INTERVAL ENOPOINTS ARE IN KILOMETERS FROH THE ACCIDENT SITE, THE 5 INTERVAL ENDPOINTS ARE $3 \quad 6 \quad 112132$ RAIN INTENSITIES ARE IN MILLIMETERS OF RAIN PER HOUR, THE 3 INTENSITY BREAKPOINTS ARE

$S \quad V$ - INITIAL HEATHER CONDITIONS HITH STABILITY CLASS S AND HIHD SPEED INTERVAL $V$

STABILITY CLASSES ARE $B=A / B, D=C / D, E=E$, AND $F=F$
HIND SPEED INTERVALS ARE IN METERS PER SECOND $(H / S), 1(0-1$

HIND SPEED INTERVALS ARE IN METERS PER SECOND (H/S), 1 (0-1), 2 (1-2), $3(2-3), 4(3-5), 5$ (5-7), 6 (GT 7 )

10.9247

10.7991

6.5297

6.2100

7.5114

1.6553

0.1256

7.6256

8.6530

6.8379

8.8356

8.6644
1.6553

1.6553

4.0183

0.4680

1.1758

1.54498

0.8447

0.8447

0.1826

0.2740

0.3082

0.1941

0.0342

0.0571

0.0799

0.2169

0.0114

0.0571

0.1598
0.0913

0.0913 


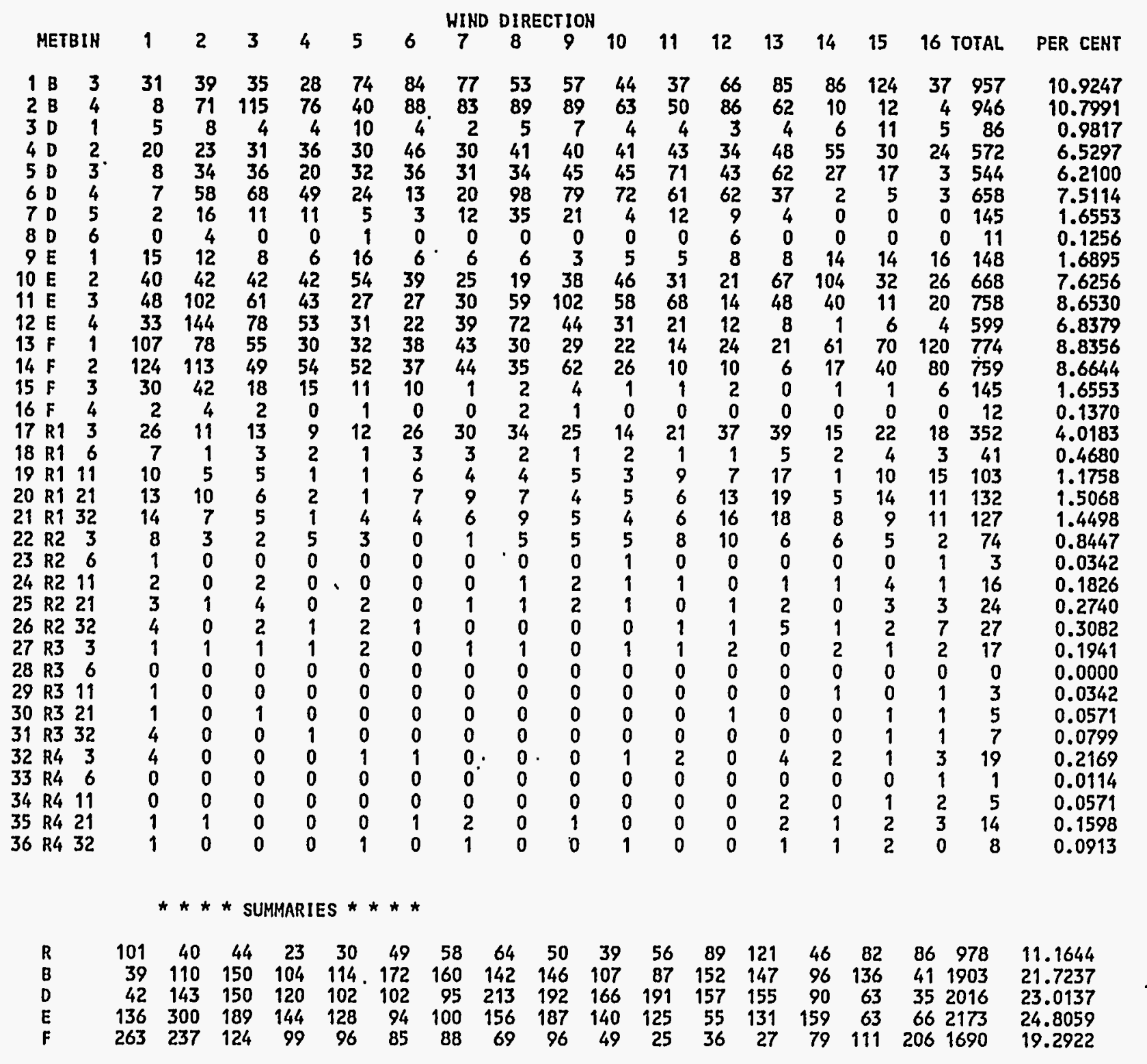




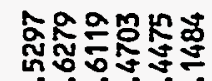
至出的。

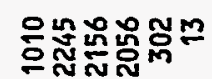

三帒品=00

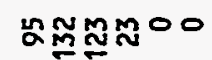

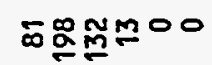

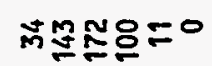

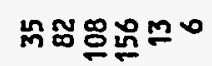

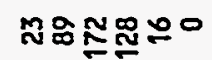

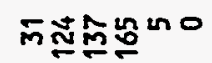

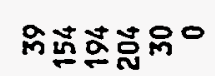

ษ哭芯出

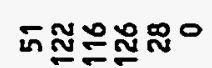

象正品す゚

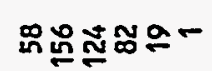

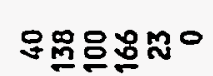

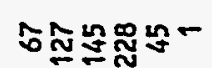

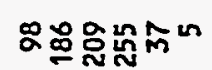

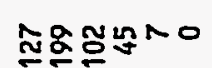

-Nmento

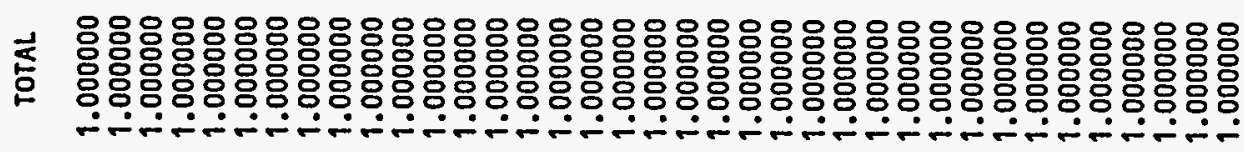

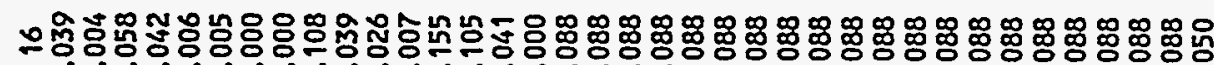
ódó0ं

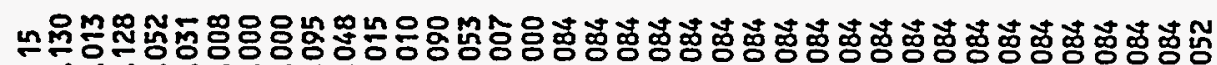
ó0ं00

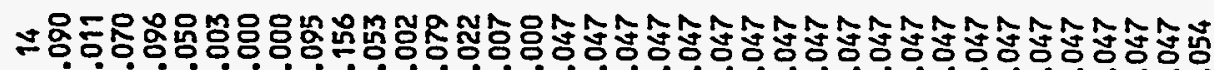

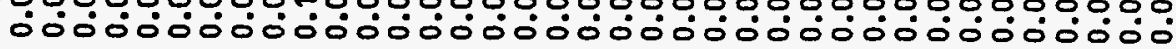

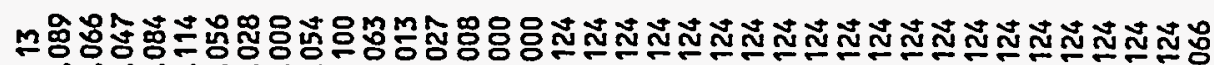

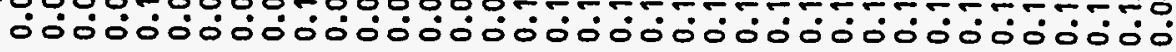

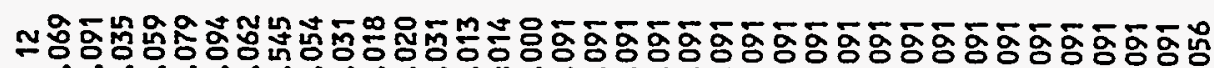

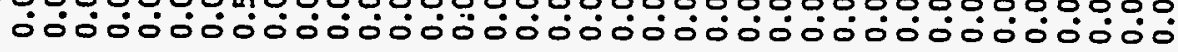

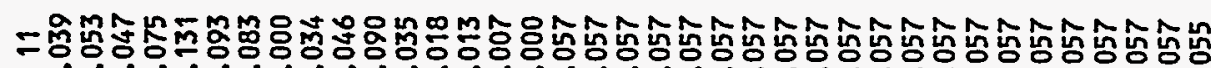

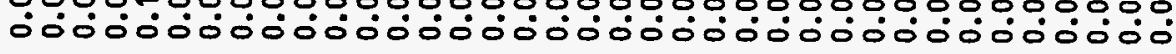

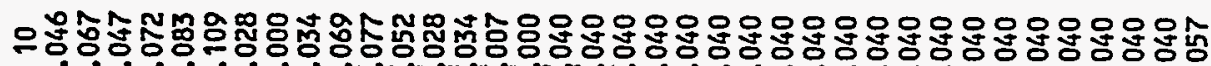

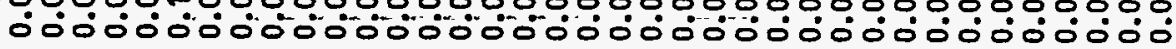

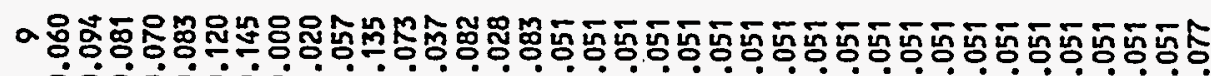

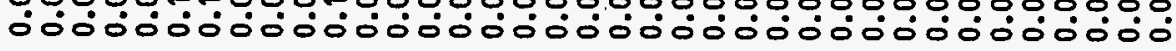

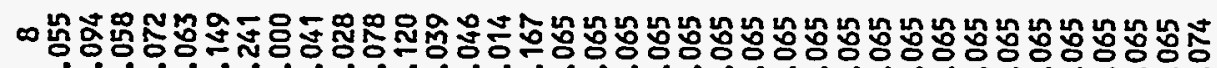

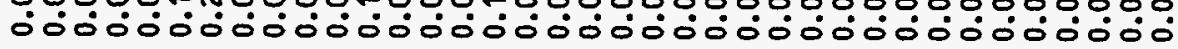

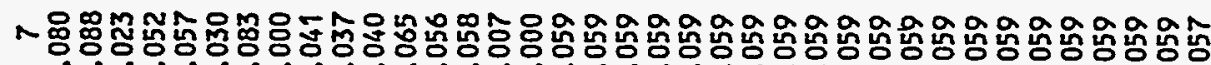

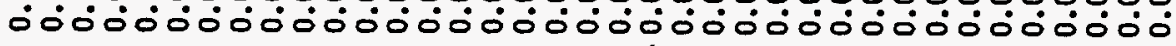

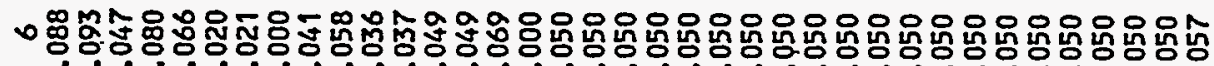

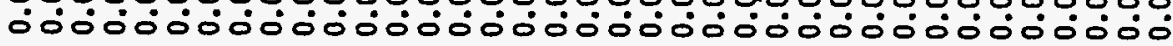

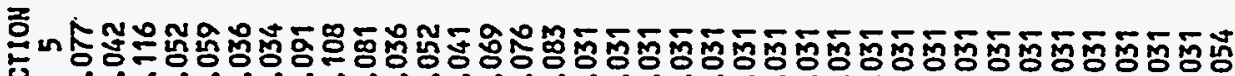
यु 00000000000000000000000000000000000

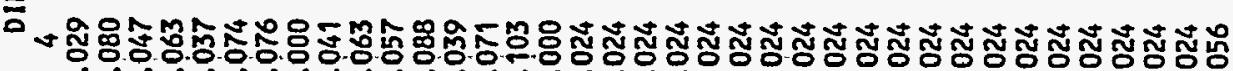

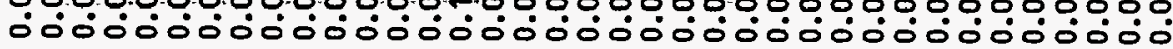

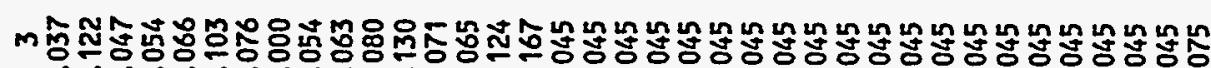

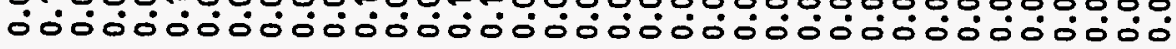

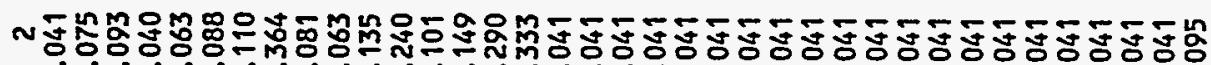

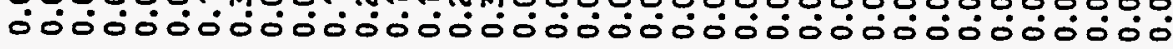

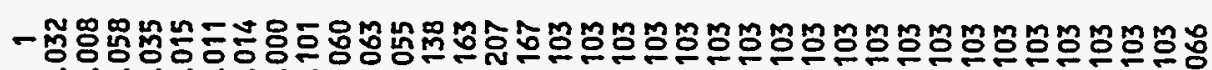

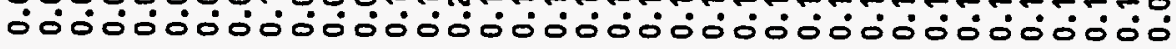

졿

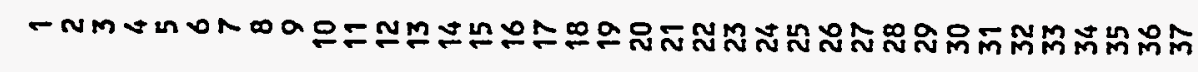


USER INPUT IS READ FRON UHIT 25

RECORD IDENTIFIER FIELDS 11 CHARACTERS LONG ARE EXPECTED.

THE FIRST 100 COLUMNS OF EACH INPUT RECORD ARE PROCESSED.

THE HAXIMUM HUHBER OF IDEHTIFIER RECORDS THAT MAY BE SAVED AS THE BASE CASE IS 1000.

RECORD

NUMBER

RECORD

* general descriptive title describing this "EarLy" INPUt file

1 MIEANAM1001 'VX_A_2.INP, CHEM_MACCS EXAMPLE PROBLEH VX_A, EARLY INPUT'

* dispersion model option code: 1 * stratght lime

* 2 * HIND-shift HITH ROtation

MIIPLUMEO01 1 (STRAIGHT LIHE PLUME)

MI

* humber of fine grid subdivisions used by the model.

3 MINUMFINOOI 7 (3, 5 OR 7 ALLOHED)

* Level of debug output required, normal runs should specify zero

4 MIIPRINTO01 0 (TURN OFF THE DEBUG PRINT)

* Logical flag signifyiǹg that the breakdown of Risk by heather category

* bin aRe to be pREsented to shoh their relative contribution to the meal

* RISBIN

MIRISCATO01 . FALSE.

$\underset{*}{*}$ MIRISCATOOI . FALSE.

* Flag INDICATINg IF hIND-ROSES fron atmos are to be overRIDDEN

6 MIOVRRI0001 fFALSE. (USE THE HIND ROSE CALCULATED FOR EACH HEATHER BIN)

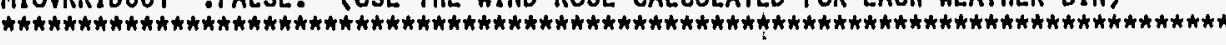
* POPULATION DISTRIBUTION DATA BLOCK, LOADED BY INPOPU, STORED IN /POPDAT/

7 PDPOPFLGO01 UNIFORM

PDIBEGINOOI 1 (SPATIAL INTERVAL. AT HHICH POPULATION BEGINS)

9 PDPOPDENO01 50. (POPULATION DENSITY (PEOPLE PER SQUARE KILOMETER))

* DOSE OEFINITION DATA BLOCK, LOADED BY INORGA, STORED IN /EARDIM/ AND /ORGNAM/

* NUMBER OF dOSES DEFINED fOR hEALTH EFFECTS 
10 ODNUMORGOO1 4

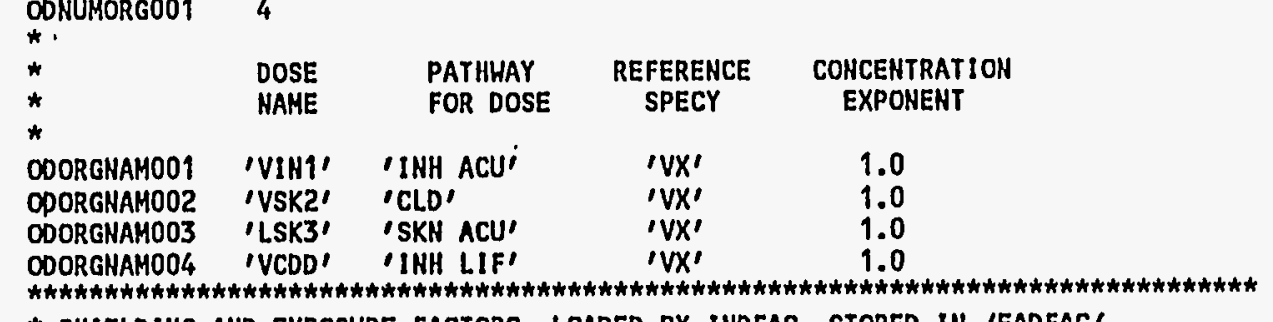

$\star \star \star n$
* SHIELDING AND EXPOSURE FACTORS, LOADED BY INDFAC, STORED IN /EADFAC/

*

* three values of each protection factor are supplied,

* ONE FOR EACH TYPE OF ACTIVTY:

$\star$

* ACTIVITY TYPE:

* 1 : EVACUEES HHILE MOVING

* $\quad 2$ : EVACUEES HHILE MOVING

* 3 - SHELTERED ACTIVITY

* protection factor for inhalation

*

15 SEPROTINO01

1. 0.41

$\star$

* breathing rate and reference breathing rate (CUbIC meters per second)

16 SEBRRATE001 $2.66 \mathrm{E}-4 \quad 2.66 \mathrm{E}-4 \quad 2.66 \mathrm{E}-4$ * BREATHING RATE

17 SEBRRATEO02 $2.66 \mathrm{E}-4 \quad 2.66 \mathrm{E}-4$ 2.66E-4 * REFERENCE BREATHIHG RATE

*

PROTECTION FACTOR

* VALUES FOR NORHAL ACTIVITY AND SHELTERING SELECTEd BY NRC STAFF

18 SESKPFACO01 $1.0 \quad 0.41 \quad 0.33$ * FOR LIQUID

19 SESKPFACO02 $1.0 \quad 0.410 .33$ * * FOR VAPOR
* RESUSPENSion inhalation model. CONCENTRATION COEfFicient (/MEter)
RESCON $=1 . E-4$ IS APPROPRIATE FOR MECHANICAL RESUSPENSION BY VEHICLES.
RESHAF $=2.11$ DAYS CAUSES 1.E-4 TO DECAY IN ONE HEEK TO 1.E-5, THE VALUE
OF RESCON USED IN THE FIRST TERM OF THE LONG-TERM RESUSPENSION EQUATION USED IN CHRONC.
20 SERESCONOO1 1.E-4 (RESUSPENSION IS TURNED ON)
* RESUSPENSION CONCENTRATION COEFFICIENT halF-LIFE (SEC)
21 SERESHAF001 $1.82 E 5$ (2.11 DAYS) 


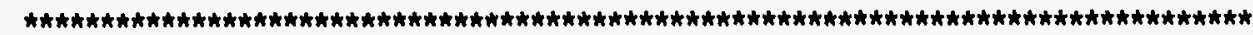

* EVACUATION ZONE DATA BLOCK, LOADED BY EVHETH, STORED IH /HETHOR/, /EOPTIO/

* Specific description of the ehergency response scehario being USEd

22 ezeanam2001 'EVACUATIOH hithin 10 miles, relocation hODELS apply ElsehHere' $\star$

* THE TYPE OF MEIGHTIHG TO BE APPLIED TO THE EMERGENCY RESPONSE SCEHARIOS

* YOU mUST SUPPLY a VALUE OF 'TIME' OR 'PEOPLE'

23 EZHTHAMEOOI 'PEOPLE'

* heighting fraction applicable to this scenario

24 EZHTFRAC001 0.95

* Last ring in the movement zone

* LAst RINQ in the MOVEMENT zoNe

25 EZLASMOVOOI 15 (EVACUEES DISAPPEAR AFTER TRAVELING TO 20 MILES)

* first spatial interval in the evacuation zone

EZINIEVA001 1 (NO INNER SHELTER ZONE)

26

* outer bounds on 3 evacuation zones (zero means the zone is not defined)

27 EZLASEVAOO

0 0 12 (SINGLE EVACUATION ZONE OUT TO 10 MILES)

* evacuation delar times for the 3 evacuation zones

* THIS IS THE DELAY TIME FROM OALARM (ATMOS) TO HHEN PEOPLE START MOVING

28 EZEDELAYO01 0. 0. 7200. (SURRY)

* radial eVAcuation SPEed (M/S)

*

29 EZESPEED001 1.8

\section{(SURRY)}

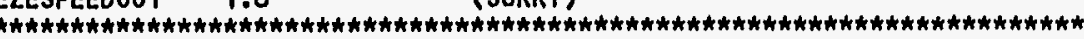

* Shelter and Relocation zONE DATA block, lOADED by INPEMr,

* STELER AND RELOCATION ZONE DATA BLOCK, LORED IN /INPSRZ/, /RELOCA/

* time to take shelter IN the inNer shelter zONe (SECONOS froh oalarh)

SRTTOSH1001 O. (THERE IS NO INNER SHELTER ZOME)

30 SRTTOSH1001 O. (THERE IS NO INNER SHELTER ZONE)

* Shelter duration IN the INNER Shelter zone (SECONDS froh taking shelter)

31 SRSHELTT1001 0 . (THERE IS NO INMER SHELTER ZONE) 
* Last ring of the outer shelter zone

32. SRLASHE2001 0 (THERE IS NO OUTER SHELTER ZONE)

* time to take shelter in the outer shelter zone (SECONds from oalarm)

33 SRTTOSH2001 0 . (THERE IS NO OUTER SHELTER ZONE)

* Shelter duration In the OUter shelter zone (SEConds frow taking shelter)

34 SRSHELT2001 0. (THERE IS NYO OUTER SHELTER ZONE)

* DURATION OF THE EMERGEHCY PHASE (SECOHDS fROM PLUME aRRIVAL)

*

35 SRENDEMP001 604800. (ONE HEEK)

* CRITICAL dOSE FOR RELOCATION DECISIONS

*

36 SRCRIORGOO1 'VSK2'

* HOt SPOT RELOCATION TIME (SECONDS FROM PLUME arRival)

37 SRTIMHOTOO1 43200 . (ONE-HALF DAY)

* nORMAL RELOCATION TIME (SECONDS froN PLUME ARRIVAL)

*

38 SRTIMHRHO01 86400. (ONE DAY)

* HOT SPOT RELOCATION DOSE CRITERION THRESHOLD (SIEVERTS)

*

39 SRDOSHOTOO 0.5 (50 REM DOSE TO HHOLE BODY IN 1 HEEK TRIGGERS RELOCATION)

* normal relocation dose CRITERION threshold

*

40 SRDOSNRMO01 0.045 (25 MG/M^3-S VAP AIR CONC DOSE IN 1 WK TRIGGERS RELOCATION)

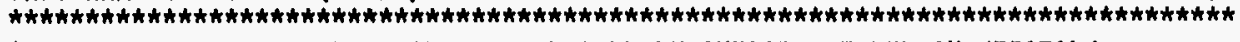

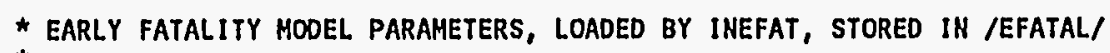

$\star$

* number of early fatality effects

41 EFNUMEFAOO1 3

$\begin{array}{lr}{ }_{\star}^{*} & \\ \star & \end{array}$

* orgnam effthr

2 EFATAGRPOO1 'VIN1'

43 EFATAGRPO02 'VSK2'

44 EFATAGRPO03 'LSK3'

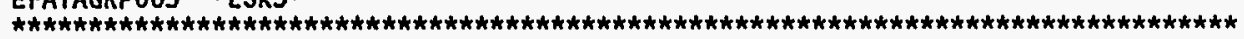


* early injury model parameters, loaded by INeinj, stored in /Einjur/

* number of early injury effects

45 EINUMEIHOO1 3

* ${ }^{\star}$ EIHAME ORGNAM EISUSC EITHRE

46 EINJUGRPOO1 'CLP, PRL, CONVL' 'LSK3'

47 EINJUGRPOO2 'HIOSIS/RHINORRH' 'VINI'

48 EINJUGRPOO3 'SWEATIHG/FASCIC' 'VSK2'

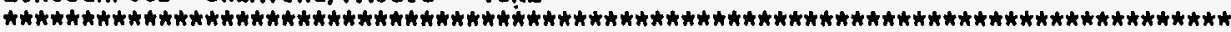

* acute exposure cancer parameters, loaded by INACAN Stored In /acancr/.

* number of acute exposure cancer effects

49

HUMACAOO1 1

*

50

LDDTHRE001 0

DOSE THRESHOLO

* DOSE tHREshold fOR LINEAR doSe RESPONSE

51 LCACTHRE001 0.

*

* achame 'orgnam acsusc dosefa dosefB cFrisk cirisk dDREFa

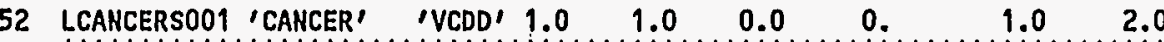

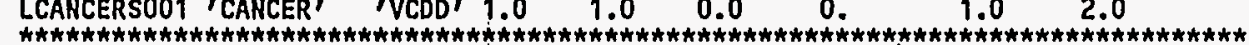
* RESULT 1 OPTIONS BLOCK, LOADED BY INOUT1, STORED IN /INOUT1/

* total. NUMBer of a GIVEN EFFECt (LATENT CÁNCER, EARLY DEATH, EARLy INJURY)

*

* NUMBer of desired ResUlts of this tYPE

53 TYPE1NUMBER 5

54 TYPE1OUTO01 'ERL. FAT/TOTAL'

55 TYPE1OUTO02 'ERL. INJ/CLP, PRL, CONVL'

56 TYPEIOUTO03 'ERL INJ/MIOSIS/RHINORRH'

57 TYPEIOUTO04 'ERL INJ/SWEATING/FASCIC'

58 TYPE1OUTO09 'CAN INJ/CANCER'

* RESULT 2 OPTIONS BLOCK, LOADED BY INOUT2, STORED IN /INOUT2/

* FURTHEST DISTANCE AT WIIICH A GIVEN RISK OF EARLY DEATH IS EXCEEDED.

*

* number of desired results of this type

126 CCDF (O TO 1000 MILES)

26

26

26
26 
60 TYPEZOUT001 0.001 CCDF *DISTANCE AT HHICH ANY FATALITIES OCCURRED

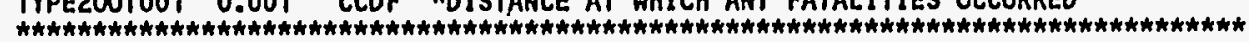
* RESULT 3 OPTIONS BLOCK, LOADED BY INOUT3, STORED IN /INOUT3/

* NUMBER OF PEOPLE HHOSE DOSE EXCEEDS A GIVEN THRESHOLD.

*

* nUMBer of desired ReSULtS of this tYPE

61 TYPE3NUMBER 4
* DOSE
* NAME
DOSE
62 TYPE3OUT001
63 TYPE3OUTO02 IYSK2
TYPE3 UUTO03
'VSK2,
THRESHOLD
IVCDD
8.22
100.

65 TYPE3OUT004

TYPE30UT004 'VCDD' 0.0

* RESULT 4 OPTIONS BLOCK, LOADED BY INOUT4, STORED IN / INOUT4/

* 360 degree aVerage risk of a given effect at a given distance.

*

* POSSIBLE TYPES OF EFFECTS ARE:

* 'erl fat/totalí

* 'erR INJ/INJURY NaME'

* ican fat/cancer hame

* 'CAN Fat/TOtal'

* NUMBER OF DESIRED RESULTS OF THIS TYPE.

*

66 TYPEGNUMBER

*

*

67 TYPE4OUTOO1

68 TYPE4OUTO02

69 TYPE4OUTO03

70 TYPELOUTO04

*TYPE4OUTO05

RADIAL INDEX TYPE OF EFFECT

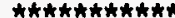

* RESULT 5 OPTIOHS BLOCK, LOADED BY INOUT5, STORED IN /INOUTS/

* total population dose betheen tho distances.

* nUMBER of DESIRED RESULTS OF THIS TYPE 
* DOSE I1DIS5 I2DIS5

72 TYPE5OUT001 'VCDD' 1 - 12 (0-10 MILES)

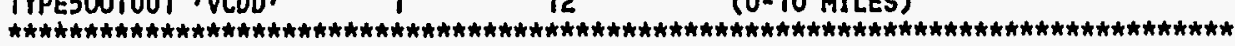

* RESULT 6 OPTIONS BLOCK, LOADED BY INOUT6, STORED IN /INOUT6/,

* Centerline dose versus distance by pathhay, pathhay names are as follohs:

* pathhar hame:

* 'ClD' NAME: - ClOUDSHINE

'GRO' - GROUNDSHINE

'INH ACU' - "ACUTE DOSE EQUIVALENT" FROM DIRECT IHHALATIOH OF THE CLOUD

'INH LIF' - "LIFETIME DOSE COMHITHENT" FROH DIRECT INHALATION OF THE CLOUD

'RES ACU' - "ACUTE DOSE EQUIVALENT" FRON RESUSPENSION INHALATION

'RES LIF' - "LIFETIME DOSE COMMITHENT" FROM RESUSPENSION INHALATION

'TOT ACU' - "ACUTE DOSE EQUIVALENT" FROM ALL PATHHAYS

'TOT LIF' - "LIFETIME DOSE COMMITMENT" FROH ALL. PATHHAYS

* humber of desired results of this type

73 TYPEGNUMBER

74

TYPE6OUTO01 'LSK3' 'TOT ACU' 1 19 (0-50 MILES)

*TYPE6OUTO03 'VCDD' 'TOT LIF' 1 ' 26 (0-1000 MILES)

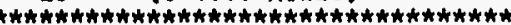

* RESULT 7 OPTIONS BLOCK, LOADED BY INOUT7, STORED IN /INOUT7/

* centerline risk of a given effect vs distance

* number of desired results of this type

75 TYPETNUMBER

* NAME L I0IS7 I I20IS7

76 TYPETOUTO0 1 'ERL FAT/TOTAL'

$190-50$ MILES)

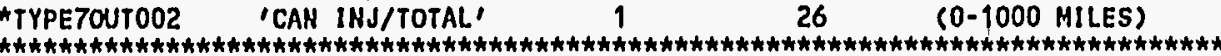

* RESULT 8 OPTIONS BLOCK, LOADED BY INOUTB, STORED IN /INOUT8/

* POPULATIOH hEIGHTED FATALITY RISK BETHEEN 2 DISTANCES

* humber of desired results of this tYPe 


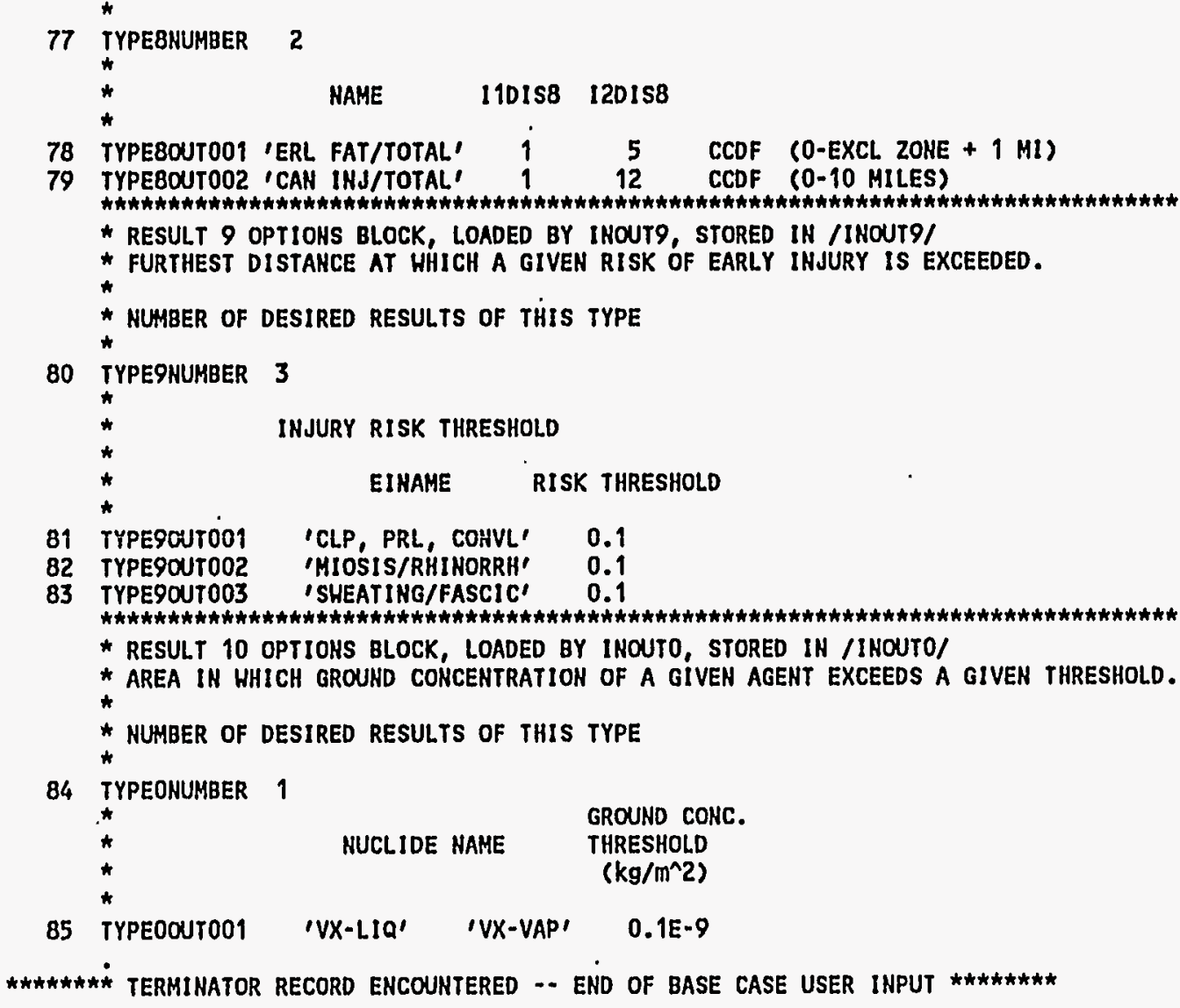




\section{CALCULATING A UMIFORM POPULATION DISTRIBUTION}

READIHG FROH A DOSE CONVERSIOH FILE HITH THE FOLLOHIHG HEADER

CHEM_MACCS FIle DOSDATA.INP: Changed by E. HASKIH 10APR95

Dose conversion factors for CHEH_MACCS Version 1.0

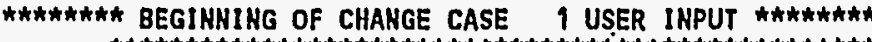

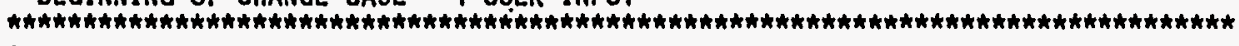

* EHERGENCY RESPOHSE SCEMARIO NUMBER 2

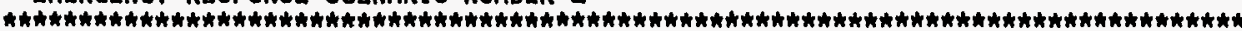

* EVACUATION zONE DATA Block, LOAded BY EVHETh, STORED IN /NEThOR/, /EOPTIO/

* SPECIFIC DESCRIPTION OF THE EHERGENCY RESPOHSE SCENARIO BEIHG USED

86 EZEANAMZOO1 'NO EVACUATION, RELOCATION MODELS APPLY EVERYWHERE'

$\star \star \star \star \star \star \star * \star$ RECORD NUMBER B6 REPLACES RECORD NUMBER 22

* HeIGHTING fraction aPPLICABLE to thIS scENARIO

* heighting fraction applicable to this scenario

87 EZHTFRACO01 0.05

RECORD NUMBER 87 REPLACES RECORD NUMBER 24

* Last RING IN THE MOVEMENT zONE

*

88 EZLASMOVOOI 0 (A ZERO TURHS OFF THE EVACUATION MODEL)

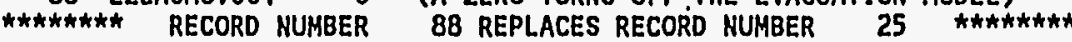

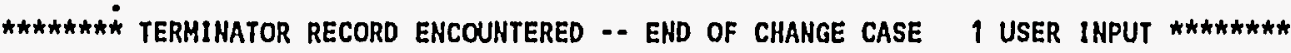

USER IHPUT PROCESSING SUMMARY - CHANGE CASE 1

$\begin{array}{ll}\text { NUMBER OF RECORDS CHANGED } & =3 \\ \text { NUMBER OF RECORDS ADDED } & =0\end{array}$

NUMBER OF RECORDS ADDED

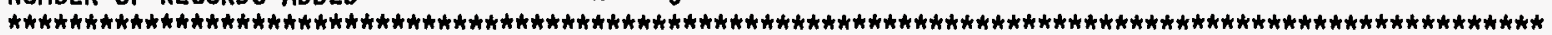

NO EVACUATION REQUESTEO

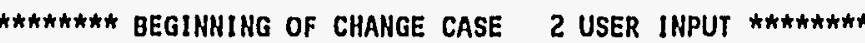

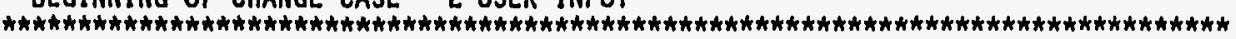

* eHERGENCY RESPONSE SCENARIO NUMBER 3

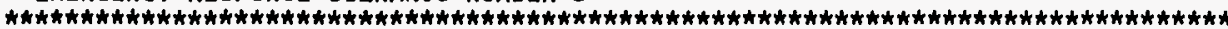

* EVACUATION ZONE DATA BLOCK, LOADED BY EVNETH, STORED IN /NETHOR/, /EOPTIO/ 
* Specific description of the emergency response scenario being used

EZEAHAM2001 ISHELTERIHG HITHIY 10 MILES, RELOCATION MODELS APPLY ELSEHHERE' 89 E2 REPLACES RECORD NUMBER 22 \%

*

* heightimg fraction applicable to this scenario

90 EŻHTFRACO01 0.0 (THIS CASE IS NOT BEING CONBINED HITH SCENARIOS 1 AND 2)

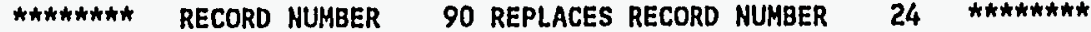

* time to take shelter IN the outer shelter zONE (SECONDS from OALARM)

91 SRTTOSH2001 2700. (45 MINUTES TO TAKE SHELTER)

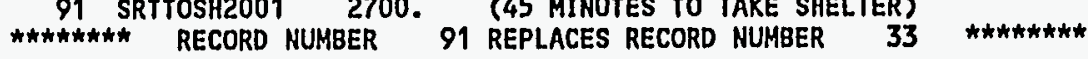

* shelter duration in the outer shelter zone (seconds froh taking shelter)

92 SRSHELT2001 43200. (12 HOUR SHELTER DURATION)

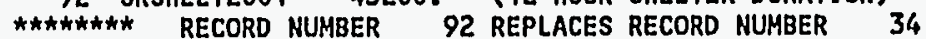

* LAST RIHG of the OUter shelter zONE

93 SRLASHE2001 12 (OUTER SHELTER ZONE EXTENDS FROM 0 TO 10 MILES)

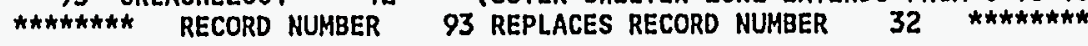

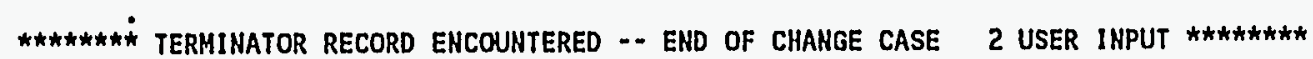

USER INPUT PROCESSING SUMMARY : CHANGE CASE 2

NUMBER OF RECORDS CHANGED $=5$

NUMBER OF RECORDS ADDED

NO EVACUATION REQUESTED

1 THIS PROGRAH CURRENTLY ALLOWS THE GENERATION OF UP TO 394 RESULTS

YOU HAVE REQUESTED 59 RESULTS FROM "EARLY" COMPOSED OF:

$$
\begin{aligned}
& 5 \text { RESULTS OF TYPE } 1 \\
& 1 \text { RESULTS OF TYPE } 2 \\
& 4 \text { RESULTS OF TYPE } 3 \\
& 4 \text { RESULTS OF TYPE } 4 \\
& 1 \text { RESULTS OF TYPE } 5 \\
& 19 \text { RESULTS OF TYPE } 6 \\
& 19 \text { RESULTS OF TYPE } 7 \\
& 2 \text { RESULTS OF TYPE } 8 \\
& 3 \text { RESULTS OF TYPE } 9 \\
& 1 \text { RESULTS OF TYPE } 10
\end{aligned}
$$




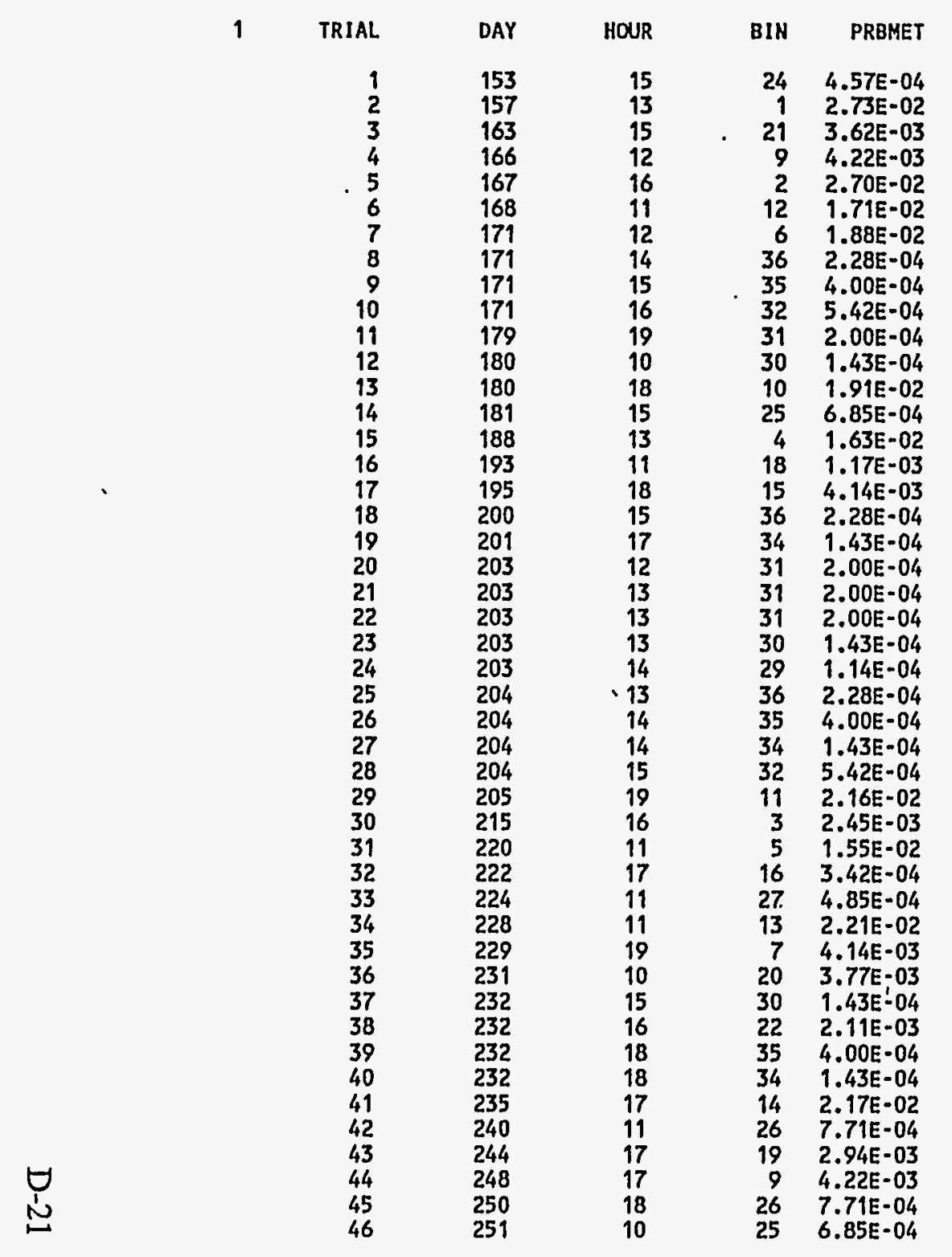


$\forall$
$N$

$\begin{array}{rrrrr}47 & 253 & 10 & 13 & 2.21 E-02 \\ 48 & 263 & 16 & 17 & 1.00 E-02 \\ 49 & 268 & 13 & 4 & 1.63 E-02 \\ 50 & 270 & 11 & 14 & 2.17 E-02 \\ \text { TRIAL } & \text { DAY } & \text { HOUR } & \text { BIN } & \text { PRBMET } \\ & & & & \\ 51 & 271 & 17 & 10 & 1.91 E-02 \\ 52 & 272 & 13 & 1 & 2.73 E-02 \\ 53 & 282 & 12 & 18 & 1.17 E-03 \\ 54 & 286 & 18 & 24 & 4.57 \mathrm{E}-04 \\ 55 & 288 & 13 & 2 & 2.70 \mathrm{E}-02 \\ 56 & 299 & 13 & 32 & 5.42 \mathrm{E}-04 \\ 57 & 299 & 14 & 27 & 4.85 \mathrm{E}-04 \\ 58 & 301 & 10 & 11 & 2.16 \mathrm{E}-02 \\ 59 & 301 & 15 & 5 & 1.55 \mathrm{E}-02 \\ 60 & 307 & 11 & 6 & 1.88 \mathrm{E}-02 \\ 61 & 309 & 14 & 21 & 3.62 \mathrm{E}-03 \\ 62 & 309 & 15 & 19 & 2.94 \mathrm{E}-03 \\ 63 & 309 & 17 & 9 & 4.22 \mathrm{E}-03 \\ 64 & 311 & 16 & 3 & 2.45 \mathrm{E}-03 \\ 65 & 315 & 17 & 20 & 3.77 \mathrm{E}-03 \\ 66 & 319 & 11 & 10 & 1.91 \mathrm{E}-02 \\ 67 & 321 & 13 & 4 & 1.63 \mathrm{E}-02 \\ 68 & 330 & 13 & 19 & 2.94 \mathrm{E}-03 \\ 69 & 330 & 13 & 18 & 1.17 \mathrm{E}-03 \\ 70 & 330 & 16 & 36 & 2.28 \mathrm{E}-04 \\ 71 & 335 & 18 & 8 & 3.14 \mathrm{E}-04 \\ 72 & 335 & 19 & 8 & 3.14 \mathrm{E}-04 \\ 73 & 336 & 12 & 22 & 2.11 \mathrm{E}-03 \\ 74 & 336 & 17 & 23 & 1.14 \mathrm{E}-04 \\ 75 & 342 & 16 & 3 & 2.45 \mathrm{E}-03 \\ 76 & 346 & 14 & 1 & 2.73 \mathrm{E}-02 \\ 77 & 347 & 18 & 11 & 2.16 \mathrm{E}-02 \\ 78 & 348 & 13 & 9 & 4.22 \mathrm{E}-03 \\ 79 & 357 & 17 & 17 & 1.00 \mathrm{E}-02 \\ 80 & 358 & 14 & 22 & 2.11 \mathrm{E}-03 \\ 81 & 358 & 15 & 27 & 4.85 \mathrm{E}-04 \\ 82 & 358 & 16 & 32 & 5.42 \mathrm{E}-04 \\ 83 & 365 & 16 & 3 & 2.45 \mathrm{E}-03 \\ 84 & 8 & 16 & 5 & 1.55 \mathrm{E}-02 \\ 85 & 9 & 12 & 15 & 4.14 \mathrm{E}-03 \\ 86 & 12 & 18 & 12 & 1.71 \mathrm{E}-02 \\ 87 & 12 & 19 & 16 & 3.42 E-04 \\ 88 & 13 & 11 & 14 & 2.17 \mathrm{E}-02 \\ 89 & 15 & 13 & 2 & 2.70 \mathrm{E}-02 \\ 90 & 23 & 13 & 7 & 4.14 \mathrm{E}-03 \\ 91 & 25 & 12 & 20 & 3.77 \mathrm{E}-03 \\ 92 & 25 & 13 & 18 & 1.17 \mathrm{E}-03 \\ & & & & \end{array}$




$\begin{array}{rrrrr}93 & 29 & 15 & 17 & 1.00 E-02 \\ 94 & 32 & 11 & 13 & 2.21 E-02 \\ 95 & 37 & 16 & 8 & 3.14 E-04 \\ 96 & 47 & 14 & 4 & 1.03 E-02 \\ 97 & 48 & 10 & 16 & 3.42 E-04 \\ 98 & 49 & 13 & 26 & 7.71 E-04 \\ 99 & 50 & 16 & 24 & 4.57 E-04 \\ 99 & 51 & 17 & 5 & 1.55 E-02 \\ 100 & \text { PRBMET }\end{array}$

$\begin{array}{lllrl}101 & 53 & 10 & 6 & 1.88 \mathrm{E}-02 \\ 102 & 53 & 14 & 21 & 3.62 \mathrm{E}-03 \\ 103 & 57 & 18 & 17 & 1.00 \mathrm{E}-02 \\ 104 & 59 & 17 & 13 & 2.21 \mathrm{E}-02 \\ 105 & 65 & 15 & 7 & 4.14 \mathrm{E}-03 \\ 106 & 69 & 10 & 12 & 1.71 \mathrm{E}-02 \\ 107 & 71 & 19 & 25 & 6.85 \mathrm{E}-04 \\ 108 & 72 & 11 & 25 & 6.85 \mathrm{E}-04 \\ 109 & 72 & 11 & 23 & 1.14 \mathrm{E}-04 \\ 110 & 72 & 15 & 20 & 3.77 \mathrm{E}-03 \\ 111 & 74 & 17 & 23 & 1.14 \mathrm{E}-04 \\ 112 & 78 & 13 & 8 & 3.14 \mathrm{E}-04 \\ 113 & 83 & 16 & 6 & 1.88 \mathrm{E}-02 \\ 114 & 84 & 17 & 10 & 1.91 \mathrm{E}-02 \\ 115 & 85 & 10 & 14 & 2.17 \mathrm{E}-02 \\ 116 & 87 & 17 & 15 & 4.14 \mathrm{E}-03 \\ 117 & 101 & 11 & 16 & 3.42 \mathrm{E}-04 \\ 118 & 107 & 15 & 19 & 2.94 \mathrm{E}-03 \\ 119 & 107 & 17 & 21 & 3.62 \mathrm{E}-03 \\ 120 & 112 & 18 & 7 & 4.14 \mathrm{E}-03 \\ 121 & 115 & 14 & 2 & 2.70 \mathrm{E}-02 \\ 122 & 122 & 17 & 11 & 2.16 \mathrm{E}-02 \\ 123 & 124 & 18 & 15 & 4.14 \mathrm{E}-03 \\ 124 & 125 & 12 & 12 & 1.71 \mathrm{E}-02 \\ 125 & 127 & 16 & 26 & 7.71 \mathrm{E}-04 \\ 126 & 133 & 19 & 24 & 4.57 \mathrm{E}-04 \\ 127 & 134 & 10 & 22 & 2.11 \mathrm{E}-03 \\ 128 & 139 & 18 & 35 & 4.00 \mathrm{E}-04 \\ 129 & 139 & 18 & 34 & 1.43 \mathrm{E}-04 \\ 130 & 139 & 19 & 33 & 1.14 \mathrm{E}-04 \\ 131 & 140 & 10 & 30 & 1.43 \mathrm{E}-04 \\ 132 & 140 & 11 & 29 & .1 .14 \mathrm{E}-04 \\ 133 & 140 & 12 & 27 & 4.85 \mathrm{E}-04 \\ 134 & 140 & 17 & 29 & 1.14 \mathrm{E}-04 \\ 135 & 151 & 13 & 1 & 2.73 \mathrm{E}-02\end{array}$

1 DATE AND TIME OF RUN = MACCS 06/23/95 12:09:59 CHEM MACCS VERSION 1S, F. ERIC HASKIN, 2/3/95 "ATMOS" DESCRIPTION = VX_A_1.1NP, CHEH_MACCS EXAMPLE PROBLEH VX_A, ATHOS INPUT "EARLY" DESCRIPTION = VX_A_2.INP, CHEM_MACCS EXAMPLE PROBLEH VX_A, EARLY INPU 
SOURCE TERM i OF 1:

CHEH_MACCS EXAMPLE SOURCE TERM VX

OVERALL. RESULTS OBTAINED BY COMBINING 3 EMERGENCY RESPONSE COHORTS FRON "EARLY" HITH THE WEIGHTING FRACTIONS BELOW APPLIED TO THEM:

FRACTIOH OF THE PEOPLE

$$
0.950
$$

COHORTT 1 = EVACUATIOH HITHIN $10^{\circ}$ MILES, RELOCATION MODELS APPLY ELSEHHERE

COHORT 2 = NO EVACUATIOH, RELOCATION MODELS APPLY EVERYHHERE

0.000

COHORT 3 = SHELTERING HITHIN 10 MILÉS, RELOCATION MODELS APPLY ELSEHHERE

\begin{tabular}{|c|c|c|c|c|c|c|c|c|c|c|c|}
\hline 12:09:59 & 1 & $\begin{array}{c}\text { PROB } \\
\text { NON-ZERO }\end{array}$ & MEAN & 50TH & $\begin{array}{l}\text { QUANT } \\
\text { 9OTH }\end{array}$ & $\begin{array}{l}\text { IILES } \\
\text { 95TH }\end{array}$ & 99TH & $99.9 \mathrm{TH}$ & $\begin{array}{l}\text { PEAK } \\
\text { COHS }\end{array}$ & $\begin{array}{l}\text { PEAK } \\
\text { PROB }\end{array}$ & $\begin{array}{l}\text { PEAK } \\
\text { TRIAL }\end{array}$ \\
\hline $\begin{array}{l}\text { HEALTH EFFECTS CASES } \\
\text { ERL FAT/TOTAL } \\
\text { ERL INJ/CLP, PRL, CONVL } \\
\text { ERL INJ/MIOSIS/RHINORRH } \\
\text { ERL INJ/SWEATING/FASCIC } \\
\text { CAN INJ/CANCER }\end{array}$ & $\begin{array}{l}0-1609 \mathrm{KM} \\
0-1609 \mathrm{KM} \\
0-1609 \mathrm{KM} \\
0-1609 \mathrm{KM} \\
0-1609 \mathrm{KM}\end{array}$ & $\begin{array}{l}1.0000 \\
1.0000 \\
1.0000 \\
1.0000 \\
1.0000\end{array}$ & $\begin{array}{l}1.32 E+01 \\
3.65 E+01 \\
1.97 E+01 \\
3.49 E-02 \\
1.24 E-06\end{array}$ & $\begin{array}{l}2.41 E+00 \\
1.72 E+00 \\
8.23 E-01 \\
1.67 E-03 \\
1.08 E-06\end{array}$ & $\begin{array}{l}3.80 E+01 \\
3.84 E+01 \\
7.80 E+00 \\
1.01 E-01 \\
1.63 E-06\end{array}$ & $\begin{array}{l}6.42 E+01 \\
7.24 E+01 \\
9.48 E+00 \\
1.24 E-01 \\
1.94 E-06\end{array}$ & $\begin{array}{l}8.06 E+01 \\
\text { HOT-FOUND } \\
\text { NOT-FOUHD } \\
\text { NOT-FOUND } \\
\text { NOT-FOUND }\end{array}$ & $\begin{array}{l}9.89 E+01 \\
\text { NOT-FOUND } \\
\text { NOT-FOUND } \\
\text { NOT-FOUND } \\
\text { NOT-FOUND }\end{array}$ & $\begin{array}{l}7.14 E+02 \\
2.42 E+03 \\
1.72 E+03 \\
1.03 E+00 \\
2.58 E-06\end{array}$ & $\begin{array}{l}1.14 E-04 \\
1.00 E-02 \\
1.00 E-02 \\
1.00 E-02 \\
2.16 E-02\end{array}$ & $\begin{array}{l}74 \\
79 \\
79 \\
79 \\
77\end{array}$ \\
\hline $\begin{array}{l}\text { EARLY FATALITY DISTAHCE (KM } \\
\text { ERL FAT/TOTAL RISK }>0.001\end{array}$ & , & 1.0000 & $2.39 E+00$ & $6.29 E-01$ & $7.29 E+00$ & $1.08 \mathrm{E}+01$ & $1.37 E+01$ & NOT-FOUND & $1.56 E+01$ & $4.14 E-03$ & 3123 \\
\hline $\begin{array}{l}\text { POPULATION EXCEEDING DOSE } \\
\text { ERL ACU VINI }>8.220 \mathrm{mg}-\mathrm{mI} \\
\text { ERL ACU VSK2 }>100.000 \mathrm{mg}-\mathrm{m} \\
\text { ERL. ACU LSK3 }>2.500 \mathrm{mg} / \mathrm{mE} \\
\text { ERL ACU VCOD }>0.000\end{array}$ & $\lim _{\min / m 3}$ & $\begin{array}{l}1.0000 \\
0.5134 \\
1.0000 \\
0.0000\end{array}$ & $\begin{array}{r}5.00 E+00 \\
8.70 E-02 \\
\cdot 1.80 E+02 \\
0.00 E+00\end{array}$ & $\begin{array}{l}1.36 E+00 \\
7.44 E-03 \\
6.92 E+00 \\
0.00 E+00\end{array}$ & $\begin{array}{l}1.35 E+01 \\
3.10 E-01 \\
8.73 E+02 \\
0.00 E+00\end{array}$ & $\begin{array}{l}1.84 E+01 \\
3.35 E-01 \\
1.35 E+03 \\
0.00 E+00\end{array}$ & $\begin{array}{l}2.29 E+01 \\
4.03 E-01 \\
2.04 E+03 \\
0.00 E+00\end{array}$ & $\begin{array}{r}2.84 E+01 \\
\text { HOT }- \text { FOUND } \\
2.16 E+03 \\
0.00 E+00\end{array}$ & $\begin{array}{l}5.11 E+01 \\
4.50 E-01 \\
2.29 E+03 \\
0.00 E+00\end{array}$ & $\begin{array}{l}4,57 E-04 \\
3.77 E-03 \\
1: 14 E-04 \\
0.00 E+00\end{array}$ & $\begin{array}{rr}14 & 54 \\
13 & 65 \\
14 & 111 \\
10 & 0\end{array}$ \\
\hline $\begin{array}{l}\text { AVERAGE INDIVIDUAL RISK } \\
\text { ERL. FAT/TOTAL } \\
\text { ERL. INJ/CLP, PRL, CONVL. } \\
\text { ERL. INJ/MIOSIS/RHINORRH } \\
\text { ERL INJ/SWEATING/FASCIC }\end{array}$ & $\begin{array}{l}0-0.2 \mathrm{KM} \\
0.2-0.5 \mathrm{KM} \\
0.5-1.2 \mathrm{KM} \\
1.2-1.6 \mathrm{KM}\end{array}$ & $\begin{array}{l}1.0000 \\
1.0000 \\
0.5677 \\
0.0828\end{array}$ & $\begin{array}{l}2.08 \mathrm{E}-01 \\
3.45 \mathrm{E}-02 \\
5.41 \mathrm{E}-03 \\
1.22 \mathrm{E}-05\end{array}$ & $\begin{array}{l}2.01 E-01 \\
2.65 E-02 \\
9.05 E-10 \\
0.00 E+00\end{array}$ & $\begin{array}{l}2.38 E-01 \\
6.39 E-02 \\
2.15 E-02 \\
0.00 E+00\end{array}$ & $\begin{array}{l}2.56 E-01 \\
7.33 E-02 \\
2.44 E-02 \\
2.51 E-11\end{array}$ & $\begin{array}{l}\text { NOT-FOUND } \\
\text { NOT-FOUND } \\
\text { NOT-FOUND } \\
\text { NOT-FOUND }\end{array}$ & $\begin{array}{l}\text { NOT-FOUND } \\
\text { NOT-FOUND } \\
\text { NOT-FOUND } \\
\text { NOT-FOUND }\end{array}$ & $\begin{array}{l}2.72 E-01 \\
9.70 E-02 \\
5.94 E-02 \\
1.22 E-03\end{array}$ & $\begin{array}{l}2.73 \mathrm{E}-02 \\
1.55 \mathrm{E}-02 \\
1.00 \mathrm{E}-02 \\
1,00 \mathrm{E}-02\end{array}$ & $\begin{array}{r}2 \\
31 \\
79 \\
79\end{array}$ \\
\hline $\begin{array}{l}\text { POPULATION DOSE (SV) } \\
\text { VCDD TOT LIF }\end{array}$ & $0-16.1 \mathrm{kM}$ & 1.0000 & 1.32E-07 & $5.33 E-08$ & 5.07E-07 & $5.98 E-07$ & NOT-FOUND & NOT-FOUND & $8.96 E-07$ & $2.16 \mathrm{E}-02$ & 77 \\
\hline $\begin{array}{ll}\text { CENTERLINE } & \text { DOSE AT SOHE DIS } \\
\text { LSK3 } & \text { TOT ACU } \\
\text { LSK3 } & \text { TOT ACU } \\
\text { LSK3 } & \text { TOT ACU } \\
\text { LSK3 } & \text { TOT ACU }\end{array}$ & $\begin{array}{r}\text { STANCES (SV) } \\
0-0.2 \mathrm{KM} \\
0.2-0.5 \mathrm{KM} \\
0.5-1.2 \mathrm{KM} \\
1.2-1.6 \mathrm{KM}\end{array}$ & $\begin{array}{l}1.0000 \\
1.0000 \\
1.0000 \\
1.0000\end{array}$ & $\begin{array}{l}1.92 E+02 \\
3.81 E+01 \\
1.21 E+01 \\
6.42 E+00\end{array}$ & $\begin{array}{l}1.70 E+02 \\
8.84 E+00 \\
1.40 E+00 \\
7.74 E-01\end{array}$ & $\begin{array}{l}3.20 E+02 \\
1.10 E+02 \\
3.92 E+01 \\
2.25 E+01\end{array}$ & $\begin{array}{l}3.40 E+02 \\
1.22 E+02 \\
5.03 E+01 \\
2.74 E+01\end{array}$ & $\begin{array}{l}3.90 E+02 \\
1.57 E+02 \\
6.02 E+01 \\
3.39 E+01\end{array}$ & $\begin{array}{r}4.75 E+02 \\
\text { NOT-FOUND } \\
\text { NOT-FOUND } \\
4.20 E+01\end{array}$ & $\begin{array}{l}5.03 E+02 \\
1.81 E+02 \\
6.65 E+01 \\
4.52 E+01\end{array}$ & $\begin{array}{l}5.42 E-04 \\
4.11 E-03 \\
4.11 E-03 \\
4.57 E-04\end{array}$ & $\begin{array}{ll}4 & 56 \\
3 & 68 \\
3 & 68 \\
4 & 54\end{array}$ \\
\hline
\end{tabular}

RESULTS, HHICH ARE PRODUCED ONLY BY "EARLY" OR ONLY BY "CHRONC" ARE PRESENTED IN LATER SECTIONS. 


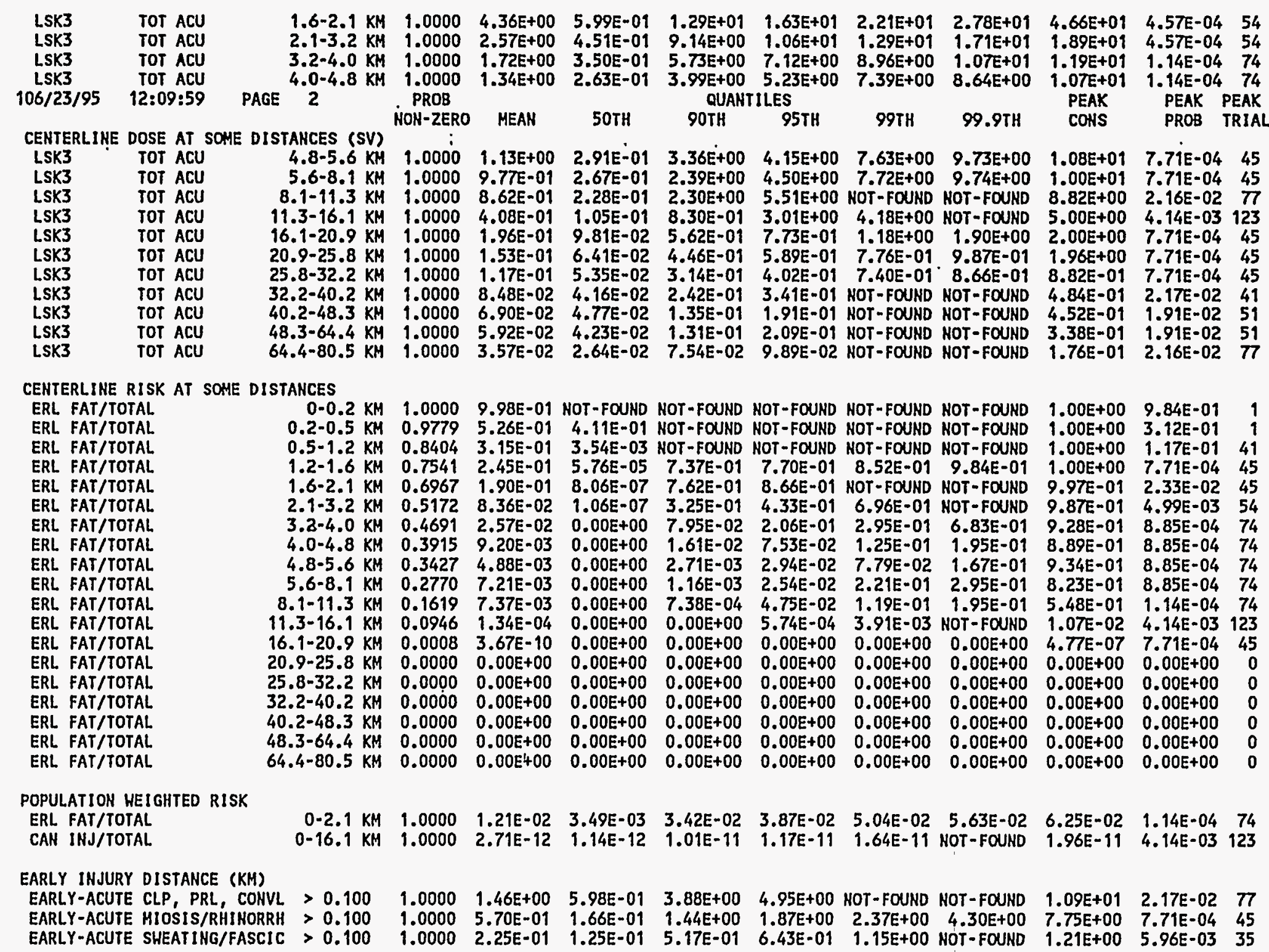


AREA DEPOSITED AGENT EXCEEDS (KMZ)

EARLY-ACUTE VX-L.IQ >.100E-09 kg/m2

"ATMOS" DESCRIPTION $\approx$ VX_A_1.INP, CHEM HACCS EXAMPLE PROBLEM VX_A, ATHOS INPUT

"EARLY" DESCRIPTION = VX_A_2.INP, CHEM_MACCS EXAMPLE PROBLEM VX_A, EARLY INPUT

SOURCE TERH 1 OF 1:

CHEM_MACCS EXAMPLE SOURCE TERH VX

RESULTS FOR A SINGLE EMERGENCY RESPONSE COHORT HITHOUT ANY WEIGHTING FRACTIONS BEING APPLIED

COHORT 1 = EVACUATION HITHIN 10 MILES, RELOCATION MOOELS APPLY ELSEWHERE

\begin{tabular}{|c|c|c|c|c|c|c|c|c|c|c|c|}
\hline $12: 09: 59$ & 3 & $\begin{array}{c}\text { PROB } \\
\text { NOH-ZERO }\end{array}$ & MEAN & 50TH & $\begin{array}{l}\text { QUANT } \\
\text { 9OTH }\end{array}$ & $\begin{array}{l}\text { 95TH } \\
\text { 95TH }\end{array}$ & 99TH & 99.9TH & $\begin{array}{l}\text { PEAK } \\
\text { CONS }\end{array}$ & $\begin{array}{l}\text { PEAK } \\
\text { PROB }\end{array}$ & $\begin{array}{l}\text { PEAK } \\
\text { TRIAL }\end{array}$ \\
\hline $\begin{array}{l}\text { HEALTH EFFECTS CASES } \\
\text { ERL FAT/TOTAL } \\
\text { ERL INJ/CLP, PRL, CONVL } \\
\text { ERL INJ/MIOSIS/RHINORRH } \\
\text { ERL. INJ/SWEATING/FASCIC } \\
\text { CAN INJ/CANCER }\end{array}$ & $\begin{array}{l}0-1609 \mathrm{KH} \\
0-1609 \mathrm{KM} \\
0-1609 \mathrm{KH} \\
0-1609 \mathrm{KH} \\
0-1609 \mathrm{KM}\end{array}$ & $\begin{array}{l}1.0000 \\
1.0000 \\
1.0000 \\
0.9933 \\
1.0000\end{array}$ & $\begin{array}{l}1.32 E+01 \\
3.77 E+01 \\
2.06 E+01 \\
3.45 E-02 \\
1.24 E-06\end{array}$ & $\begin{array}{l}2.36 E+00 \\
1.65 E+00 \\
8.07 E-01 \\
9.92 E-04 \\
1.09 E-06\end{array}$ & $\begin{array}{l}3.78 E+01 \\
3.84 E+01 \\
7.80 E+00 \\
1.01 E-01 \\
1.77 E-06\end{array}$ & $\begin{array}{l}6.42 \mathrm{E}+01 \\
7.25 \mathrm{E}+01 \\
9.48 \mathrm{E}+00 \\
1.24 \mathrm{E}-01 \\
2.14 \mathrm{E}-06\end{array}$ & $\begin{array}{l}8.19 E+01 \\
\text { NOT-FOUND } \\
\text { NOT-FOUND } \\
\text { NOT-FOUND } \\
\text { NOT-FOUND }\end{array}$ & $\begin{array}{l}1.63 E+02 \\
\text { NOT-FOUND } \\
\text { NOT-FOUND } \\
\text { NOT-FOUND } \\
\text { NOT-FOUND }\end{array}$ & $\begin{array}{l}7.49 E+02 \\
2.54 E+03 \\
1.81 E+03 \\
1.08 E+00 \\
2.61 E-06\end{array}$ & $\begin{array}{l}1.14 E-04 \\
1.00 E-02 \\
1.00 E-02 \\
1.00 E-02 \\
2.16 E-02\end{array}$ & $\begin{array}{l}74 \\
79 \\
79 \\
79 \\
77\end{array}$ \\
\hline $\begin{array}{l}\text { EARLY FATALITY DISTANCE (KM } \\
\text { ERL FAT/TOTAL RISK }>0.001\end{array}$ & & 1.0000 & $2.39 E+00$ & $6.29 E-01$ & $7.29 E+00$ & $1.09 E+01$ & $1.40 E+01$ & NOT-FOUND & $1.61 E+01$ & $4.14 E-03$ & 123 \\
\hline $\begin{array}{l}\text { POPULATION EXCEEDING DOSE } \\
\text { ERL ACU VINI }>8.220 \mathrm{mg}-\mathrm{mi} \\
\text { ERL ACU VSK2 }>100.000 \mathrm{mg}-\mathrm{m} \\
\text { ERL ACU LSK3 > }>2.500 \mathrm{mg} / \mathrm{mb} \\
\text { ERL ACU VCDD }>0.000\end{array}$ & $\begin{array}{l}\operatorname{lin}_{\mathrm{min} / \mathrm{m} 3} \\
\text { an }\end{array}$ & $\begin{array}{l}0.9975 \\
0.3819 \\
1.0000 \\
0.0000\end{array}$ & $\begin{array}{l}4.78 E+00 \\
8.19 E-02 \\
1.86 E+02 \\
0.00 E+00\end{array}$ & $\begin{array}{l}1.27 E+00 \\
0.00 E+00 \\
6.18 E+00 \\
0.00 E+00\end{array}$ & $\begin{array}{l}1.20 E+01 \\
3.07 E-01 \\
8.73 E+02 \\
0.00 E+00\end{array}$ & $\begin{array}{l}1.45 E+01 \\
3.24 E-01 \\
1.46 E+03 \\
0.00 E+00\end{array}$ & $\begin{array}{l}2.11 E+01 \\
3.69 E-01 \\
2.07 E+03 \\
0.00 E+00\end{array}$ & $\begin{array}{r}2.80 E+01 \\
\text { NOT-FOUND } \\
2.23 E+03 \\
0.00 E+00\end{array}$ & $\begin{array}{l}5.30 E+01 \\
3.95 E-01 \\
2.40 E+03 \\
0.00 E+00\end{array}$ & $\begin{array}{l}4.57 E-04 \\
4.31 E-03 \\
1.14 E-04 \\
0.00 E+00\end{array}$ & $\begin{array}{r}54 \\
56 \\
111 \\
0\end{array}$ \\
\hline $\begin{array}{l}\text { AVERAGE INDIVIDUAL RISK } \\
\text { ERL FAT/TOTAL } \\
\text { ERL. INJ/CLP, PRL, CONVL } \\
\text { ERL. INJ/HIOSIS/RHINORRH } \\
\text { ERL. INJ/SHEATING/FASCIC }\end{array}$ & $\begin{array}{rl}0-0.2 & \mathrm{KH} \\
0.2-0.5 \mathrm{KH} \\
0.5-1.2 \mathrm{KM} \\
1.2-1.6 \mathrm{KH}\end{array}$ & $\begin{array}{l}1.0000 \\
0.8577 \\
0.3674 \\
0.0414\end{array}$ & $\begin{array}{l}2.08 E-01 \\
3.36 E-02 \\
5.35 E-03 \\
1.29 E-05\end{array}$ & $\begin{array}{l}2.01 E-01 \\
2.48 E-02 \\
0.00 E+00 \\
0.00 E+00\end{array}$ & $\begin{array}{l}2.22 E-01 \\
6.39 E-02 \\
2.14 E-02 \\
0.00 E+00\end{array}$ & $\begin{array}{l}2.32 E-01 \\
7.33 E-02 \\
2.43 E-02 \\
0.00 E+00\end{array}$ & $\begin{array}{l}2.56 E-01 \\
\text { NOT-FOUND } \\
\text { NOT-FOUND } \\
\text { NOT-FOUND }\end{array}$ & $\begin{array}{l}\text { NOT-FOUND } \\
\text { NOT-FOUND } \\
\text { NOT-FOUND } \\
\text { NOT-FOUND }\end{array}$ & $\begin{array}{l}2.72 E-01 \\
9.69 E-02 \\
6.25 E-02 \\
1.28 E-03\end{array}$ & $\begin{array}{l}3.77 E-03 \\
1.55 E-02 \\
1.00 E-02 \\
1.00 E-02\end{array}$ & $\begin{array}{r}110 \\
31 \\
79 \\
79\end{array}$ \\
\hline $\begin{array}{l}\text { POPULATION DOSE (SV) } \\
\text { VCDD TOT LIF }\end{array}$ & $0-16.1 \mathrm{KM}$ & 1.0000 & $1.32 E^{\prime}-07$ & $4.81 E-08$ & $5.14 E-07$ & $6.86 E-07$ & NOT-FOUND & NOT-FOUND & $9.26 \mathrm{E}-07$ & $2.16 \mathrm{E}-02$ & 77 \\
\hline $\begin{array}{ll}\text { CENTERLINE } & \text { DOSE AT SOHE D } \\
\text { LSK3 } & \text { TOT ACU } \\
\text { LSK3 } & \text { TOT ACU } \\
\text { LSK3 } & \text { TOT ACU } \\
\text { LSK3 } & \text { TOT ACU } \\
\text { LSK3 } & \text { TOT ACU } \\
\text { LSK3 } & \text { TOT ACU } \\
\text { LSK3 } & \text { TOT ACU }\end{array}$ & $\begin{array}{r}\text { STANCES (SV) } \\
0-0.2 \mathrm{KH} \\
0.2-0.5 \mathrm{KH} \\
0.5-1.2 \mathrm{KH} \\
1.2-1.6 \mathrm{KH} \\
1.6-2.1 \mathrm{KH} \\
2.1-3.2 \mathrm{KH} \\
3.2-4.0 \mathrm{KH}\end{array}$ & $\begin{array}{l}1.0000 \\
1.0000 \\
1.0000 \\
1.0000 \\
1.0000 \\
0.9900 \\
0.9900\end{array}$ & $\begin{array}{l}1.89 E+02 \\
3.75 E+01 \\
1.18 E+01 \\
6.30 E+00 \\
4.27 E+00 \\
2.51 E+00 \\
1.69 E+00\end{array}$ & $\begin{array}{l}1.70 E+02 \\
8.80 E+00 \\
1.27 E+00 \\
6.34 E-01 \\
4.93 E-01 \\
3.63 E-01 \\
2.74 E-01\end{array}$ & $\begin{array}{l}3.19 E+02 \\
1.07 E+02 \\
3.92 E+01 \\
2.25 E+01 \\
1.29 E+01 \\
9.14 E+00 \\
5.73 E+00\end{array}$ & $\begin{array}{l}3.38 E+02 \\
1.20 E+02 \\
5.03 E+01 \\
2.74 E+01 \\
1.63 E+01 \\
1.06 E+01 \\
7.12 E+00\end{array}$ & $\begin{array}{l}3.88 E+02 \\
1.56 E+02 \\
6.01 E+01 \\
3.42 E+01 \\
2.21 E+01 \\
1.30 E+01 \\
8.96 E+00\end{array}$ & $\begin{array}{r}4.73 E+02 \\
\text { NOT-FOUND } \\
\text { NOT-FOUND } \\
4.31 E+01 \\
2.78 E+01 \\
1.73 E+01 \\
1.08 E+01\end{array}$ & $\begin{array}{l}4.98 E+02 \\
1.80 E+02 \\
6.63 E+01 \\
4.67 E+01 \\
4.81 E+01 \\
1.91 E+01 \\
1.22 E+01\end{array}$ & $\begin{array}{l}5.42 E-04 \\
4.11 \mathrm{E}-03 \\
4.11 \mathrm{E}-03 \\
4.57 \mathrm{E}-04 \\
4.57 \mathrm{E}-04 \\
4.57 \mathrm{E}-04 \\
1.14 \mathrm{E}-04\end{array}$ & $\begin{array}{l}56 \\
68 \\
68 \\
54 \\
54 \\
54 \\
74\end{array}$ \\
\hline
\end{tabular}




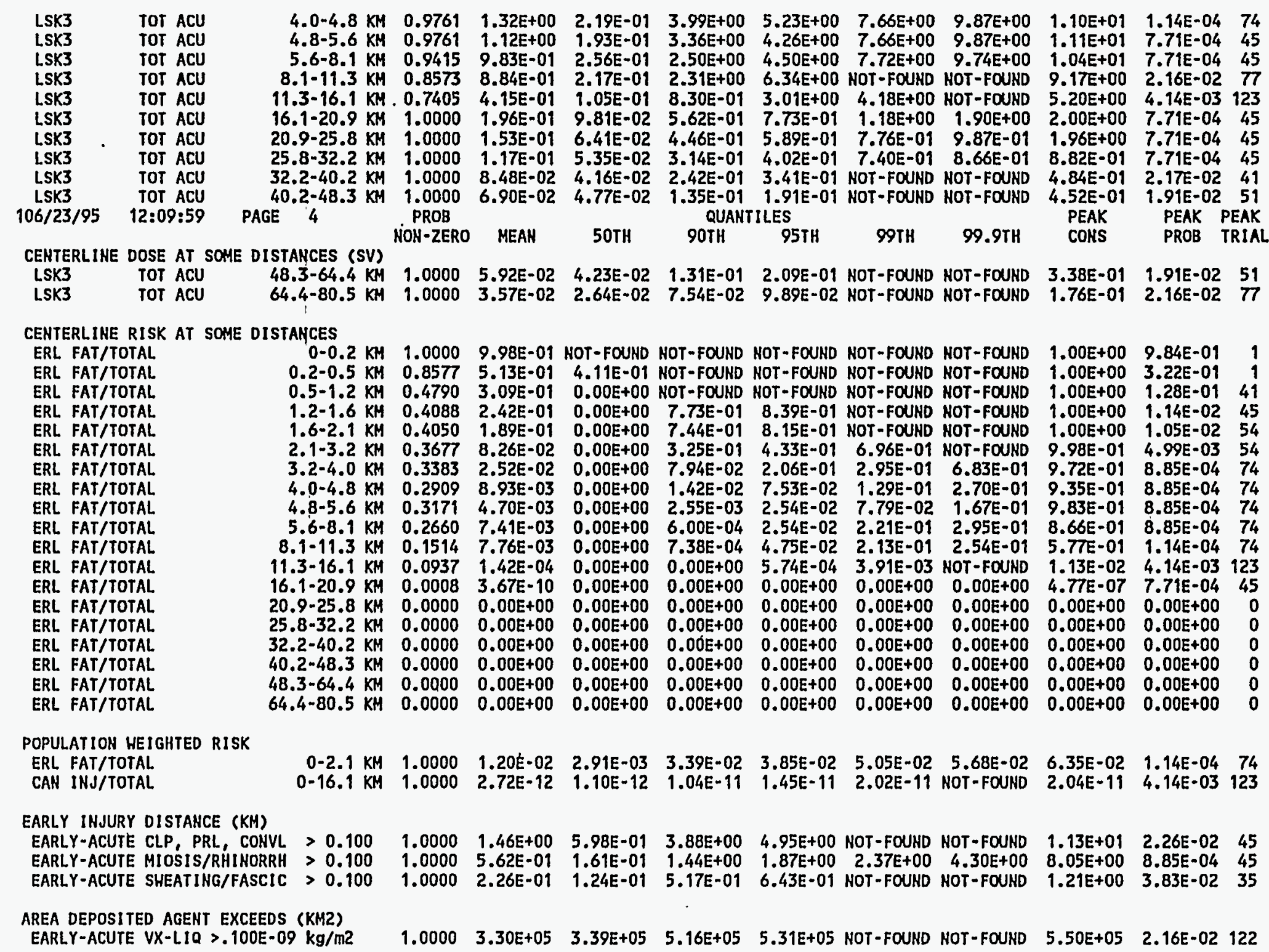


1 DATE AND TIME OF RUH = MACCS 06/23/95 12:09:59 CHEM MACCS VERSION 1S, F. ERIC HASKIH, 2/3/95 "ATMOS" DESCRIPTIOH = VX_A_1.1NP, CHEM_MACCS EXAMPLE PROBLEM VX_A, ATMOS INPUT "EARLY" DESCRIPTION = VX_A_2.INP, CHEM_MACCS EXAMPLE PROBLEM VX_A, EARLY INPUT

SOURCE TERM 1 OF 1

CHEM_HACCS EXAMPLE SOURCE TERM VX

RESULTS FOR A SINGLE EMERGENCY RESPONSE COHORT HITHOUT AHY WEIGHTING FRACTIONS BEIHG APPLIED

COHORT 2 = NO EVACUATION, RELOCATION MODELS APPLY EVERYHHERE

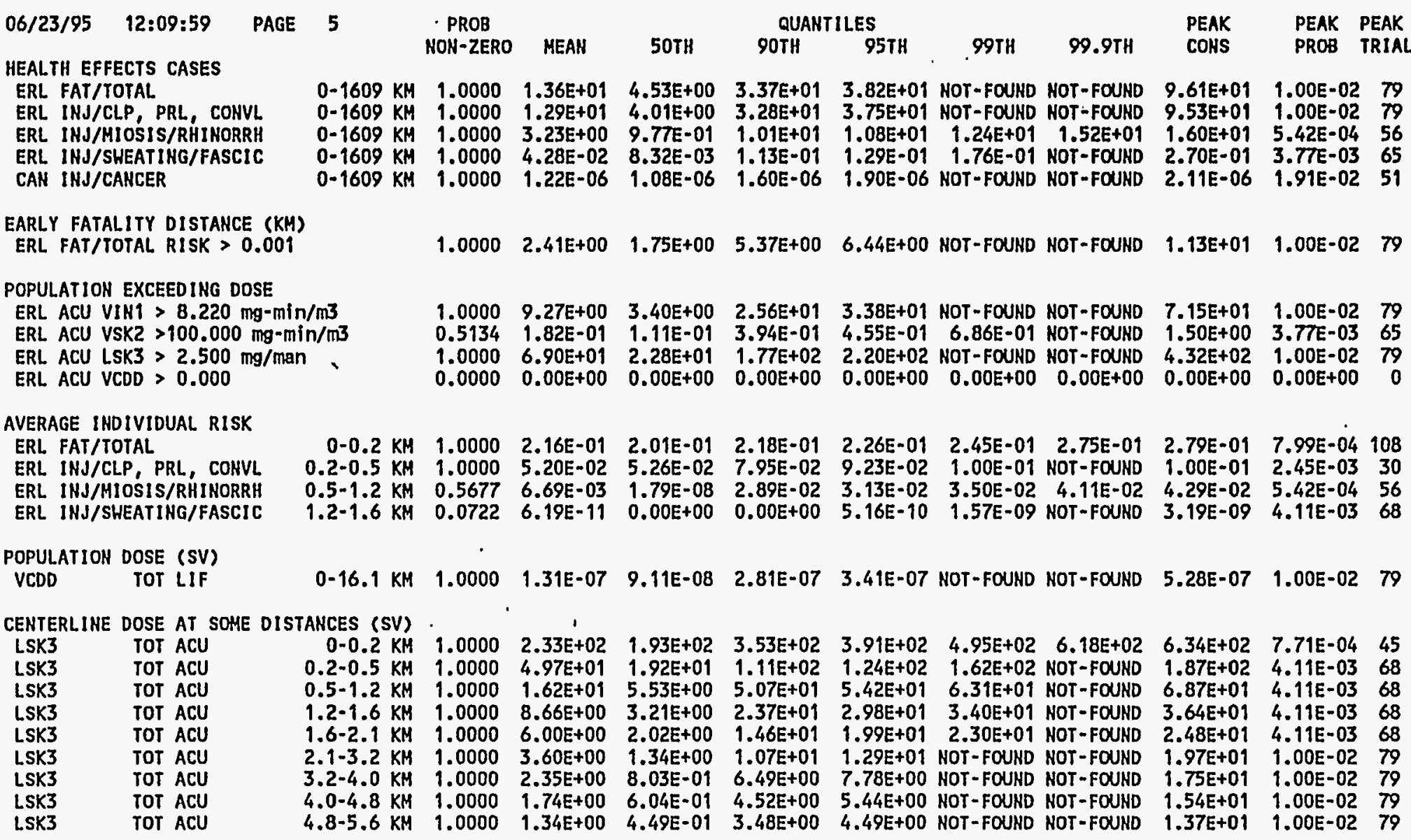




\begin{tabular}{|c|c|c|c|c|c|c|c|c|c|c|c|c|}
\hline $\begin{array}{l}\text { LSK3 } \\
\text { LSK3 } \\
\text { LSK3 } \\
\text { LSK3 } \\
\text { LSK3 } \\
\text { LSK3 } \\
\text { LSK3 } \\
\text { LSK3 } \\
\text { 106/23/95 }\end{array}$ & $\begin{array}{l}\text { TOT ACU } \\
\text { TOT ACU } \\
\text { TOT ACU } \\
\text { Tot ACU } \\
\text { TOT ACU } \\
\text { TOT ACU } \\
\text { TOT ACU } \\
\text { TOT ACU } \\
12: 09: 59\end{array}$ & $\begin{array}{rl}5.6-8.1 & \mathrm{KH} \\
8.1-11.3 \mathrm{KH} \\
11.3-16.1 \mathrm{KH} \\
16.1-20.9 \mathrm{KH} \\
20.9-25.8 \mathrm{KH} \\
25.8-32.2 \mathrm{KH} \\
32.2-40.2 \mathrm{KH} \\
40.2-48.3 \mathrm{KH} \\
\text { PAGE } 6\end{array}$ & $\begin{array}{c}1.0000 \\
1.0000 \\
1.0000 \\
1.0000 \\
.1 .0000 \\
1.0000 \\
1.0000 \\
1.0000 \\
\text { PROB } \\
\text { NON-ZERO }\end{array}$ & $\begin{array}{r}8.64 E-01 \\
4.46 E-01 \\
2.69 \mathrm{E}-01 \\
.1 .96 \mathrm{E}-01 \\
1.53 \mathrm{E}-01 \\
1.17 \mathrm{E}-01 \\
8.48 \mathrm{E}-02 \\
6.90 \mathrm{E}-02 \\
\text { MEAN }\end{array}$ & $\begin{array}{l}4.10 E-01 \\
2.11 \mathrm{E}-01 \\
1.40 \mathrm{E}-01 \\
9.81 \mathrm{E}-02 \\
6.41 \mathrm{E}-02 \\
5.35 \mathrm{E}-02 \\
4.16 \mathrm{E}-02 \\
4.77 \mathrm{E}-02 \\
50 \mathrm{TH}\end{array}$ & $\begin{array}{c}2.17 \mathrm{E}+00 \\
1.19 \mathrm{E}+00 \\
7.50 \mathrm{E}-01 \\
5.62 \mathrm{E}-01 \\
4.46 \mathrm{E}-01 \\
3.14 \mathrm{E}-01 \\
2.42 \mathrm{E}-01 \\
1.35 \mathrm{E}-01 \\
\text { QUANT } \\
\text { 90TH }\end{array}$ & $\begin{array}{r}2.72 E+00 \\
1.65 \mathrm{E}+00 \\
1.05 \mathrm{E}+00 \\
7.73 \mathrm{E}-01 \\
5.89 \mathrm{E}-01 \\
4.02 \mathrm{E}-01 \\
3.41 \mathrm{E}-01 \\
1.91 \mathrm{E}-01 \\
\text { TILES } \\
95 \mathrm{TH}\end{array}$ & $\begin{array}{r}0 \text { NOT-FOUND } \\
\text { HOT-FOUND } \\
1.24 E+00 \\
1.18 E+00 \\
7.76 E-01 \\
7.40 E-01 \\
\text { HOT-FOND } \\
\text { NOT-FOUND }\end{array}$ & $\begin{array}{r}\text { NOT-FOUND } \\
\text { NOT-FOUND } \\
1.59 E+00 \\
1.90 E+00 \\
9.87 E-01 \\
8.66 E-01 \\
\text { NOT-FOND } \\
\text { HOT-FOUND }\end{array}$ & $\begin{array}{l}1.00 E+01 \\
4.17 E+00 \\
2.28 E+00 \\
2.00 E+00 \\
1.96 E+00 \\
8.82 E-01 \\
4.84 E-01 \\
4.52 E-01 \\
\text { PEAK } \\
\text { CONS }\end{array}$ & $\begin{array}{l}1.00 E-02 \\
1.00 E-02 \\
1.14 E-04 \\
7.71 E-04 \\
7.71 E-04 \\
7.71 E-04 \\
2.17 E-02 \\
1.91 E-02 \\
\text { PEAK P } \\
\text { PROB }\end{array}$ & $\begin{array}{l}79 \\
79 \\
74 \\
45 \\
45 \\
45 \\
49 \\
51 \\
\text { PEAK } \\
\text { TRIAL }\end{array}$ \\
\hline $\begin{array}{l}\text { CENTERIINE } \\
\text { LSK3 } \\
\text { LSK3 }\end{array}$ & $\begin{array}{l}\text { DOSE AT S } \\
\text { TOT ACU } \\
\text { TOT ACU }\end{array}$ & $\begin{array}{r}\text { IE DISTANCES (SV) } \\
48.3-64.4 \mathrm{KM} \\
64.4-80.5 \mathrm{KM}\end{array}$ & $\begin{array}{l}1.0000 \\
1.0000\end{array}$ & $\begin{array}{l}5.92 E-02 \\
3.57 E-02\end{array}$ & & & & & & & & $\begin{array}{l}51 \\
77\end{array}$ \\
\hline $\begin{array}{l}\text { CENTERLIIIE } \\
\text { ERL FAT/TO } \\
\text { ERL FAT/TO } \\
\text { ERL FAT/TO } \\
\text { ERL FAT/TO } \\
\text { ERL FAT/TO } \\
\text { ERL FAT/TO } \\
\text { ERL FAT/TO } \\
\text { ERL FAT/TO } \\
\text { ERL FAT/TO } \\
\text { ERL FAT/TO } \\
\text { ERL FAT/TO } \\
\text { ERL FAT/TO } \\
\text { ERL FAT/TO } \\
\text { ERL FAT/TO } \\
\text { ERL FAT/TO } \\
\text { ERL FAT/TO } \\
\text { ERL FAT/TO } \\
\text { ERL FAT/TO } \\
\text { ERL FAT/TO }\end{array}$ & $\begin{array}{l}\text { RISK AT So } \\
\text { JTAL } \\
\text { TAL } \\
\text { TAL } \\
\text { TAL } \\
\text { TAL } \\
\text { TAL } \\
\text { TAL. } \\
\text { TAL } \\
\text { TAL } \\
\text { TAL } \\
\text { TAL } \\
\text { TAL } \\
\text { TAL } \\
\text { TAL } \\
\text { TAL }\end{array}$ & $\begin{array}{r}\text { DISTANCES } \\
0-0.2 \mathrm{KM} \\
0.2-0.5 \mathrm{KM} \\
0.5-1.2 \mathrm{KM} \\
1.2-1.6 \mathrm{KM} \\
1.6-2.1 \mathrm{KM} \\
2.1-3.2 \mathrm{KM} \\
3.2-4.0 \mathrm{KM} \\
4.0-4.8 \mathrm{KM} \\
4.8-5.6 \mathrm{KM} \\
5.6-8.1 \mathrm{KM} \\
8.1-11.3 \mathrm{KM} \\
11.3-16.1 \mathrm{KM} \\
16.1-20.9 \mathrm{KM} \\
20.9-25.8 \mathrm{KM} \\
25.8-32.2 \mathrm{KM} \\
32.2-40.2 \mathrm{KM} \\
40.2-48.3 \mathrm{KM} \\
48.3-64.4 \mathrm{KM} \\
64.4-80.5 \mathrm{KM}\end{array}$ & $\begin{array}{l}1.0000 \\
0.9779 \\
0.8404 \\
0.7370 \\
0.6575 \\
0.4780 \\
0.4299 \\
0.3682 \\
0.2640 \\
0.2047 \\
0.0818 \\
0.0009 \\
0.0008 \\
0.0000 \\
0.0000 \\
0.0000 \\
0.0000 \\
0.0000 \\
0.0000\end{array}$ & $\begin{array}{l}1.00 E+00 \\
7.67 E-01 \\
4.16 E-01 \\
3.05 E-01 \\
2.16 E-01 \\
1.02 E-01 \\
3.44 E-02 \\
1.43 E-02 \\
8.16 E-03 \\
3.39 E-03 \\
2.90 E-05 \\
1.29 E-09 \\
3.67 E-10 \\
0.00 E+00 \\
0.00 E+00 \\
0.00 E+00 \\
0.00 E+00 \\
0.00 E+00 \\
0.00 E+00\end{array}$ & $\begin{array}{l}\text { NOT-FOUAD } \\
8.51 E-01 \\
7.35 E-02 \\
1.22 E-03 \\
1.78 E-05 \\
0.00 E+00 \\
0.00 E+00 \\
0.00 E+00 \\
0.00 E+00 \\
0.00 E+00 \\
0.00 E+00 \\
0.00 E+00 \\
0.00 E+00 \\
0.00 E+00 \\
0.00 E+00 \\
0.00 E+00 \\
0.00 E+00 \\
0.00 E+00 \\
0.00 E+00\end{array}$ & $\begin{array}{l}\text { NOT-FOUND } \\
\text { NOT-FOUND } \\
\text { NOT-FOUHD } \\
7.57 E-01 \\
7.43 E-01 \\
4.15 E-01 \\
1.04 E-01 \\
2.18 E-02 \\
3.14 E-03 \\
2.27 E-05 \\
0.00 E+00 \\
0.00 E+00 \\
0.00 E+00 \\
0.00 E+00 \\
0.00 E+00 \\
0.00 E+00 \\
0.00 E+00 \\
0.00 E+00 \\
0.00 E+00\end{array}$ & $\begin{array}{c}\text { NOT-FOUND } \\
\text { NOT-FOUND } \\
\text { NOT-FOUND } \\
8.04 E-01 \\
7.90 E-01 \\
6.36 E-01 \\
1.74 E-01 \\
4.35 E-02 \\
7.86 E-03 \\
2.27 E-04 \\
7.51 E-07 \\
0.00 E+00 \\
0.00 E+00 \\
0.00 E+00 \\
0.00 E+00 \\
0.00 E+00 \\
0.00 E+00 \\
0.00 E+00 \\
0.00 E+00\end{array}$ & $\begin{array}{l}\text { NOT-FOUND } \\
\text { NOT-FOUND } \\
\text { NOT-FOUND } \\
9.25 E-01 \\
9.10 E-01 \\
9 . T \text { - } \\
\text { NOT-FOUND } \\
\text { NOT-FOUND } \\
\text { NOT-FOUND } \\
\text { NOT-FOUND } \\
\text { NOT-FOUND } \\
\text { NOT-FUND } \\
0.00 E+00 \\
0.00 E+00 \\
0.00 E+00 \\
0.00 E+00 \\
0.00 E+00 \\
0.00 E+00 \\
0.00 E+00 \\
0.00 E+00\end{array}$ & $\begin{array}{l}\text { HOT-FOUND } \\
\text { NOT-FOUND } \\
\text { NOT-FOUND } \\
\text { NOT-FOUND } \\
\text { NOT-FOUND } \\
\text { NOT-FOUND } \\
\text { NOT-FOUND } \\
\text { HOT-FOUND } \\
\text { NOT-FOUND } \\
\text { NOT-FOUND } \\
\text { NOT-FOUND } \\
0.00 E+00 \\
0.00 E+00 \\
0.00 E+00 \\
0.00 E+00 \\
0.00 E+00 \\
0.00 E+00 \\
0.00 E+00 \\
0.00 E+00\end{array}$ & $\begin{array}{l}1.00 E+00 \\
1.00 E+00 \\
1.00 E+00 \\
1.00 E+00 \\
9.85 E-01 \\
9.42 E-01 \\
8.87 E-01 \\
7.96 E-01 \\
6.77 E-01 \\
3.31 E-01 \\
2.84 E=03 \\
8.6 E-06 \\
4.77 E-07 \\
0.00 E+00 \\
0.00 E+00 \\
0.00 E+00 \\
0.00 E+00 \\
0.00 E+00 \\
0.00 E+00\end{array}$ & & $\begin{array}{r}1 \\
1 \\
41 \\
68 \\
68 \\
79 \\
79 \\
79 \\
79 \\
79 \\
79 \\
74 \\
45 \\
0\end{array}$ \\
\hline $\begin{array}{l}\text { POPULATION } \\
\text { ERL FAT/TO } \\
\text { CAN INJ/TO }\end{array}$ & & $\begin{array}{r}0-2.1 \mathrm{KH} \\
0-16.1 \mathrm{KH}\end{array}$ & $\begin{array}{l}.0000 \\
.0000\end{array}$ & & & & & $\begin{array}{r}3.89 E-02 \\
\text { NOT-FOUND }\end{array}$ & $\begin{array}{r}4.74 E-02 \\
\text { NOT-FOUND }\end{array}$ & $\begin{array}{l}5.25 E-02 \\
9.89 E-12\end{array}$ & & $\begin{array}{l}56 \\
79\end{array}$ \\
\hline $\begin{array}{l}\text { EARLY INJUR } \\
\text { EARLY-ACUT } \\
\text { EARLY-ACUT } \\
\text { EARLY-ACUT }\end{array}$ & $\begin{array}{l}\text { EIOSIS, } \\
\text { EWEATII }\end{array}$ & $\begin{array}{ll}\text { HVL } & >0.100 \\
\text { RRH } & >0.100 \\
\text { CIC }>0.100\end{array}$ & $\begin{array}{l}.0000 \\
.0000 \\
.0000\end{array}$ & $\begin{array}{l}1.52 \mathrm{E} \\
7.15 \mathrm{E} \\
2.07 \mathrm{E}\end{array}$ & & & & & & & & $\begin{array}{l}79 \\
79 \\
83\end{array}$ \\
\hline $\begin{array}{l}\text { EARLY-ACL } \\
\text { DATE AND }\end{array}$ & & 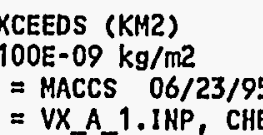 & 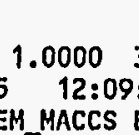 & & & & & & & 5.50E+05 & $2.16 \mathrm{E}-02$ & 122 \\
\hline
\end{tabular}


"EARLY" DESCRIPTION $=$ VX____.INP, CHEM_MACCS EXAMPLE PROBLEM VX_A, EARLY INPUT

SOURCE TERM 1 OF 1 :

CHEM_MACCS EXAMPLE SOURCE TERM VX

RESULTS FOR A SINGLE EMERGENCY RESPONSE COHORT HITHOUT ANY HEIGHTING FRACTIONS BEING APPLIED

COHORT 3 × SHELTERING HITHIN 10 MILES, RELOCATION MODELS APPLY ELSEHHERE

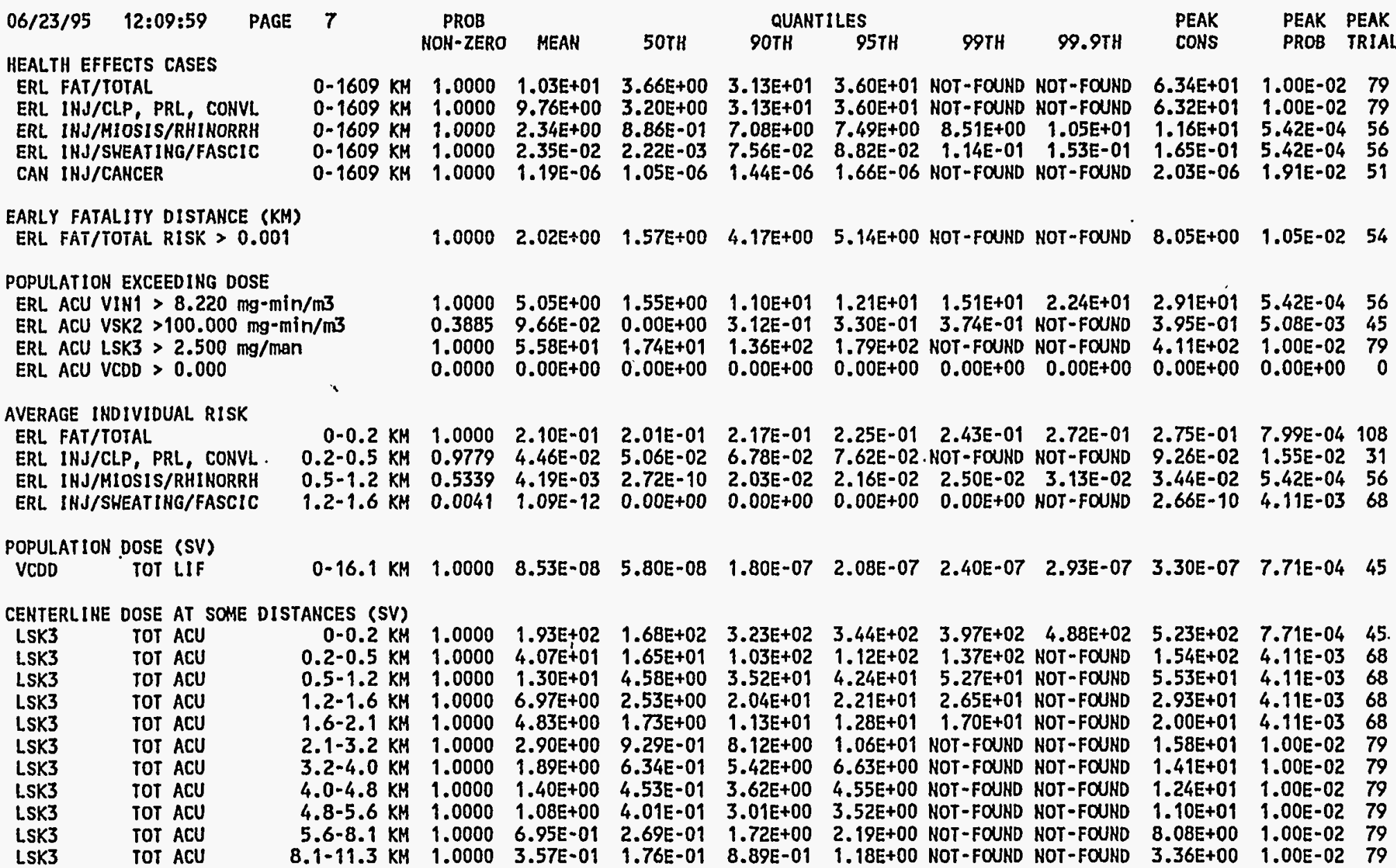




\begin{tabular}{|c|c|c|c|c|c|c|c|c|c|c|c|}
\hline $\begin{array}{l}\text { LSK3 } \\
\text { LSK3 } \\
\text { LSK3 } \\
\text { LSK3 } \\
\text { LSK3 }\end{array}$ & $\begin{array}{l}\text { TOT ACU } \\
\text { TOT ACU } \\
\text { TOT ACU } \\
\text { TOT ACU } \\
\text { TOT ACU } \\
\text { TOr ACU }\end{array}$ & $\begin{array}{l}11.3-16.1 \mathrm{KM} \\
16.1-20.9 \mathrm{KH} \\
20.9-25.8 \mathrm{KM} \\
25.8-32.2 \mathrm{KM} \\
32.2-40.2 \mathrm{KM} \\
40.2-48.3 \mathrm{KH}\end{array}$ & $\begin{array}{l}1.0000 \\
1.0000 \\
1.0000 \\
1.0000 \\
1.0000 \\
1.0000\end{array}$ & $\begin{array}{l}2.13 E-01 \\
1.96 E-01 \\
1.53 E-01 \\
1.17 E-01 \\
8.48 E-02 \\
6.90 E-02\end{array}$ & $\begin{array}{l}1.05 E-01 \\
9.81 E-02 \\
6.41 E-02 \\
5.35 E-02 \\
4.16 E-02 \\
4.77 E-02\end{array}$ & $\begin{array}{l}6.11 E-01 \\
5.62 E-01 \\
4.46 E-01 \\
3.14 E-01 \\
2.42 E-01 \\
1.35 E-01\end{array}$ & $\begin{array}{l}7.96 E-01 \\
7.73 E-01 \\
5.89 E-01 \\
4.02 E-01 \\
3.41 E-01 \\
1.91 E-01\end{array}$ & $\begin{array}{r}1.10 E+00 \\
1.18 E+00 \\
7.76 E-09 \\
7.40 E-01 \\
\text { NOT-FOUND } \\
\text { NOT-FOUND }\end{array}$ & $\begin{array}{r}1.43 E+00 \\
1.90 E+00 \\
9.87 E-01 \\
8.66 E-01 \\
\text { NOT-FOUND } \\
\text { NOT-FOUND }\end{array}$ & $\begin{array}{l}1.83 E+00 \\
2.00 E+00 \\
1.96 E+00 \\
8.82 E-01 \\
4.84 E-01 \\
4.52 E-01\end{array}$ & $\begin{array}{l}1.14 \mathrm{E}-04 \\
7.71 \mathrm{E}-04 \\
7.71 \mathrm{E}-04 \\
7.71 \mathrm{E}-04 \\
2.17 \mathrm{E}-02 \\
1.91 \mathrm{E}-02\end{array}$ \\
\hline & & PAGE 8 & $\begin{array}{c}\text { PROB } \\
\text { NON-ZERO }\end{array}$ & HEAN & & & & & & & \\
\hline $\begin{array}{l}\text { CENTER } \\
\text { LSK3 } \\
\text { LSK3 }\end{array}$ & $\begin{array}{l}\text { TOT ACU } \\
\text { TOT ACU }\end{array}$ & KM & $\begin{array}{l}1.0000 \\
1.0000\end{array}$ & & & $\begin{array}{l}1.31 E-09 \\
7.54 E-02\end{array}$ & & $\begin{array}{l}\text { FOUND } \\
\text { FOUND }\end{array}$ & $\begin{array}{l}\text { NOT-FOUND } \\
\text { NOT-FOUND }\end{array}$ & & \\
\hline
\end{tabular}

CENTERLINE RISK AT SOME DISTANCES

\begin{tabular}{|c|c|c|c|c|c|c|c|c|c|c|}
\hline $\begin{array}{l}\text { ERL } \text { FAT/TOTAL } \\
\text { ERL. FAT/TOTAL } \\
\text { ERL. FAT/TOTAL. } \\
\text { ERL. FAT/TOTAL } \\
\text { ERL. FAT/TOTAL } \\
\text { ERL FAT/TOTAL } \\
\text { ERL. FAT/TOTAL } \\
\text { ERL. FAT/TOTAL } \\
\text { ERL. FAT/TOTAL } \\
\text { ERL FAT/TOTAL } \\
\text { ERL FAT/TOTAL } \\
\text { ERL FAT/TOTAL } \\
\text { ERL. FAT/TOTAL } \\
\text { ERL. FAT/TOTAL } \\
\text { ERL FAT/TOTAL } \\
\text { ERL. FAT/TOTAL } \\
\text { ERL FAT/TOTAL } \\
\text { ERL FAT/TOTAL } \\
\text { ERL FAT/TOTAL }\end{array}$ & $\begin{array}{r}0-0.2 \mathrm{KM} \\
0.2-0.5 \mathrm{KM} \\
0.5-1.2 \mathrm{KM} \\
1.2-1.6 \mathrm{KM} \\
1.6-2.1 \mathrm{KM} \\
2.1-3.2 \mathrm{KM} \\
3.2-4.0 \mathrm{KM} \\
4.0-4.8 \mathrm{KM} \\
4.8-5.6 \mathrm{KM} \\
5.6-8.1 \mathrm{KM} \\
8.1-11.3 \mathrm{KM} \\
11.3-16.1 \mathrm{KM} \\
16.1-20.9 \mathrm{KH} \\
20.9-25.8 \mathrm{KM} \\
25.8-32.2 \mathrm{KM} \\
32.2-40.2 \mathrm{KM} \\
40.2-48.3 \mathrm{KM} \\
48.3-64.4 \mathrm{KM} \\
64.4-80.5 \mathrm{KM}\end{array}$ & $\begin{array}{l}1.0000 \\
0.9779 \\
0.8165 \\
0.6912 \\
0.5978 \\
0.4606 \\
0.4078 \\
0.2913 \\
0.2483 \\
0.1945 \\
0.0553 \\
0.0001 \\
0.0008 \\
0.0000 \\
0.0000 \\
0.0000 \\
0.0000 \\
0.0000 \\
0.0000\end{array}$ & $\begin{array}{l}1.00 E+00 \\
6.91 E-01 \\
3.84 E-01 \\
2.58 E-01 \\
1.68 E-01 \\
6.41 E-02 \\
1.86 E-02 \\
8.19 E-03 \\
5.06 E-03 \\
1.69 E-03 \\
5.02 E-06 \\
8.17 E-11 \\
3.67 E-10 \\
0.00 E+00 \\
0.00 E+00 \\
0.00 E+00 \\
0.00 E+00 \\
0.00 E+00 \\
0.00 E+00\end{array}$ & $\begin{array}{r}\text { NOT-FOUND } \\
7.76 E-01 \\
2.12 E-02 \\
1.20 E-04 \\
1.44 E-06 \\
0.00 E+00 \\
0.00 E+00 \\
0.00 E+00 \\
0.00 E+00 \\
0.00 E+00 \\
0.00 E+00 \\
0.00 E+00 \\
0.00 E+00 \\
0.00 E+00 \\
0.00 E+00 \\
0.00 E+00 \\
0.00 E+00 \\
0.00 E+00 \\
0.00 E+00\end{array}$ & $\begin{array}{c}\text { NOT-FOUND } \\
\text { NOT-FOUND } \\
9.30 E-01 \\
7.50 E-01 \\
7.39 E-01 \\
2.54 E-01 \\
4.05 E-02 \\
5.36 E-03 \\
5.94 E-04 \\
2.27 E-06 \\
0.00 E+00 \\
0.00 E+00 \\
0.00 E+00 \\
0.00 E+00 \\
0.00 E+00 \\
0.00 E+00 \\
0.00 E+00 \\
0.00 E+00 \\
0.00 E+00\end{array}$ & $\begin{array}{l}\text { NOT-FOUND } \\
\text { NOT-FOUND } \\
\text { NOT-FOUND } \\
7.97 E-01 \\
8.49 E-01 \\
4.32 E-01 \\
6.42 E-02 \\
1.36 E-02 \\
1.26 E-03 \\
2.28 E-05 \\
5.34 E-08 \\
0.00 E+00 \\
0.00 E+00 \\
0.00 E+00 \\
0.00 E+00 \\
0.00 E+00 \\
0.00 E+00 \\
0.00 E+00 \\
0.00 E+00\end{array}$ & $\begin{array}{l}\text { NOT-FOUND } \\
\text { NOT-FOUND } \\
\text { NOT-FOUND } \\
9.20 E-01 \\
\text { NOT-FOUND } \\
\text { NOT-FOUND } \\
\text { NOT-FOUND } \\
\text { NOT-FOUND } \\
\text { NOT-FOUND } \\
\text { NOT-FOUND } \\
\text { NOT-FOUND } \\
0.00 E+00 \\
0.00 E+00 \\
0.00 E+00 \\
0.00 E+00 \\
0.00 E+00 \\
0.00 E+00 \\
0.00 E+00 \\
0.00 E+00\end{array}$ & $\begin{array}{l}\text { NOT-FOUND } \\
\text { NOT-FOUND } \\
\text { NOT-FOUHD } \\
\text { NOT-FOUND } \\
\text { NOT-FOUND } \\
\text { NOT-FOUND } \\
\text { NOT-FOUND } \\
\text { NOT-FOUNO } \\
\text { NOT-FOUND } \\
\text { NOT-FOUND } \\
\text { NOT-FOUND } \\
0.00 E+00 \\
\text { O.OOE+OD } \\
\text { O.OOE+0O } \\
0.00 E+00 \\
0.00 E+00 \\
0.00 E+00 \\
0.00 E+00 \\
0.00 E+00\end{array}$ & $\begin{array}{l}1.00 E+00 \\
1.00 E+00 \\
1.00 E+00 \\
9.96 E-01 \\
9.38 E-01 \\
8.43 E-01 \\
7.45 E-01 \\
6.12 E-01 \\
4.70 E-01 \\
1.67 E-01 \\
4.95 E-04 \\
7.15 E-07 \\
4.77 E-07 \\
0.00 E+00 \\
0.00 E+00 \\
0.00 E+00 \\
0.00 E+00 \\
0.00 E+00 \\
0.00 E+00\end{array}$ & $\begin{array}{l}1.00 E+00 \\
3.34 E-01 \\
7.23 E-02 \\
4.11 E-03 \\
3.03 E-02 \\
1.00 E-02 \\
1.00 E-02 \\
1.00 E-02 \\
1.00 E-02 \\
1.00 E-02 \\
1.00 E-02 \\
1.14 E-04 \\
7.71 E-04 \\
0.00 E+00 \\
0.00 E+00 \\
0.00 E+00 \\
0.00 E+00 \\
0.00 E+00 \\
0.00 E+00\end{array}$ \\
\hline
\end{tabular}

POPULATION WEIGHTED RISK

ERL FAT/TOTAL

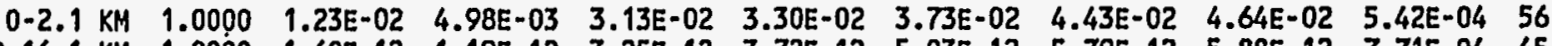
CAN INJ/TOTAL

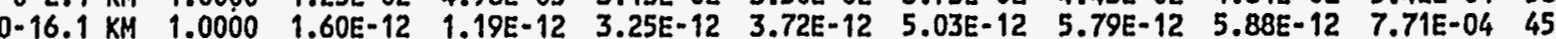

EARLY IHJURY DISTANCE (KH)

EARLY-ACUTE CLP, PRL, CONVL $>0.100 \quad 1.0000 \quad 1.35 E^{\prime}+00 \quad 6.58 E-01 \quad 3.36 E+00 \quad 3.79 E+00$ NOT-FOUND NOT-FOUND $\quad 8.05 E+00 \quad 1.00 E-02 \quad 79$ EARLY-ACUTE MIOSIS/RHIHORRH $>0.100 \quad 1.0000 \quad 5.82 E-01 \quad 1.82 E-01 \quad 1.36 E+00 \quad 1.67 E+00$ NOT-FOUND NOT-FOUND $2.13 E+00$ 2.72E-02 45 EARLY-ACUTE SHEATIHG/FASCIC $>0.100 \quad 1.0000 \quad 2.28 E-01 \quad 1.26 E-01 \quad 5.30 E-01 \quad 6.32 E-01$ NOT-FOUND NOT-FOUND $1.21 E+00$ 3.36E-02 35

\begin{abstract}
AREA DEPOSITED AGENT EXCEEDS (KM2)
EARLY-ACUTE VX-LIQ > 100E-09 kg/m2 $1.0000 \quad 3.30 E+05 \quad 3.39 E+05 \quad 5.16 E+05 \quad 5.31 E+05$ NOT-FOUND NOT-FOUND $5.50 E+05 \quad 2.16 E-02 \quad 122$ IMACCS 06/23/95 12:09:59 CHEM MACCS VERSION 1S, F. ERIC HASKIN, 2/3/95 PAGE 9
\end{abstract}

SOURCE TERM 1 OF 1 .

CHEM_MACCS EXAMPLE SOURCE TERM VX 
RESULT NAME = HEALTH EFFECTS CASES

ERL. FAT/TOTAL

$$
\text { PEOPLE FRACTION = }
$$

0.9500
EMER. RESP. \# $i$ $x$

PROB> $\times X$

$2.00 E-05 \quad 1.00 E+00$

$2.00 E-05 \quad 1.00 E+00$

$3.00 E-05 \quad 1.00 E+00$

5.00E-05 1.00E+00

7.00E-05 1.00E +00

$1.00 E-04 \quad 1.00 E+00$

2.00E-04 1.00E+00

$3.00 \mathrm{E}-04 \quad 1.00 \mathrm{E}+00$

5.00E-04 1.00E+00

$7.00 E-04 \quad 1.00 E+00$

$1.00 \mathrm{E}-03$ 1.00E+00

$2.00 \mathrm{E}-03 \quad 1.00 \mathrm{E}+00$

$3.00 E-03 \quad 1.00 E+00$

5.00E-03 1.00E+00

7.00E-03 1.00E+00

$1.00 E-02 \quad 1.00 E+00$

2.00E-02 9.98E-01

$3.00 \mathrm{E}-02 \quad 9.98 \mathrm{E}-01$

5.00E-02 9.98E-01

$7.00 E-02 \quad 9.98 E-01$

$1.00 \mathrm{E}-01 \quad 9.98 \mathrm{E}-01$

2.00E-01 9.98E-01

3.00E-01 9.93E-01

5.00E-01 9.84E-01

7.00E-01 8.58E-01

$1.00 E+00 \quad 7.86 E-01$

2.00E+00 5.94E-01

$3.00 E+00 \quad 3.90 E-01$

$5.00 E+00 \quad 3.63 E-0$

$7.00 \mathrm{E}+00$ 3.26E-01

1.00E+01 2.68E-01

2.00E+01 2.16E-01

3.00E+01 1.70E-01

$5.00 \mathrm{E}+01 \quad 5.28 \mathrm{E}-02$

7.00E+01 4.91E-02

$1.00 \mathrm{E}+02 \quad 1.34 \mathrm{E}-03$

$2.00 E+02 \quad 8.85 E-04$

$3.00 E+02 \quad 8.85 E-04$

5.00E+02 8.85E-04

$7.00 E+02 \quad 1.14 E-04$
$0-1609 \mathrm{KM}$

0.0500

0.0000

EMER. RESP. \# 2 $x \quad$ PROB> $>X$ 1.00E-05 1.00E+00 2.00E-05 1.00E+00

3.00E-05 1.00E+00

5.00E-05 1.00E+00

$7.00 \mathrm{E}-05 \quad 1.00 \mathrm{E}+00$

$1.00 E-04 \quad 1.00 E+00$

2.00E-04 1.00E+00

3.00E-04 1.00E+00

$5.00 \mathrm{E}-04 \quad 1.00 \mathrm{E}+00$

7.00E-04 1.00E+00

1.00E-03 1.00E+00

2.00E-03 1.00E+00

$3.00 E-03 \quad 1.00 E+00$

5. O0E-03 1.00E+00

7.00E-03 1.00E+00

1.00E-02 1.00E+00

2.00E-02 1.00E+00

3.00E-02 1.00E+00

5. O0E-02 1.00E+00

7.00E-02 1.00E+00

$1.00 \mathrm{E}-01 \quad 1.00 \mathrm{E}+00$

2.00E-01 1.00E+00

3.00E-01 1.00E+00

5.00E-01 1.00E+00

7.00E-01 1.00E+00

$1.00 \mathrm{E}+00 \quad 9.74 \mathrm{E}-01$

2. OOE+00 8.14E-01

3.00E+00 6.47E-01

5. $00 \mathrm{E}+00$ 4.70E-01

7.00E+00 4.09E-01

1. $00 E+01 \quad 3.82 E-01$

2.00E+01 2.64E-01

5.00E+01 1.14E-02

7.00E+01 1.00E-02

9.61E+01 1.00E-02

H.D. H.D.

N.D. H.D.

N.D. N.D.
EHER. RESP. \# 3

$X \quad P R O B>=X$

$1.00 E-06 \quad 1.00 E+00$

2.00E-06 1.00E+00

$3.00 \mathrm{E}-06 \quad 1.00 \mathrm{E}+00$

5.00E-06 1.00E+00

7.00E-06 1.00E+00

$1.00 \mathrm{E}-05 \quad 1.00 \mathrm{E}+00$

2.00E-05 1.00E+OD

3.00E-05 1.00E+00

$5.00 \mathrm{E}-05 \quad 1.00 \mathrm{E}+00$

2.00E-04 1 OOE+OO

$3.00 E-04 \quad 1.00 E+00$

5.00E-04 1.00E+00

7.00E-04 1.00E+00

1.00E-03 1.00E+00

2.00E-03 1.00E+00

3.00E-03 1.00E+00

$5.00 E-03 \quad 1.00 E+00$

$7.00 E-03 \quad 1.00 E+00$

$1.00 E-02 \quad 1.00 E+00$

2.00E-02 1.00E+00

3.00E-02 1.00E+00

5.00E-02 1.00E+00

$7.00 \mathrm{E}-02 \quad 1.00 \mathrm{E}+00$

$1.00 \mathrm{E}-01 \quad 1.00 \mathrm{E}+00$

2.00E-01 1.00E+00

3.00E-01 1.00E+00

5.00E-01 1.00E+00

7.00E-01 9.74E-01

$1.00 E+00$ 9.38E-0

$2.00 E+00 \quad 7.06 E-01$

$3.00 E+00$ 5.50E-01

5. $00 E+00 \quad 4.31 E-01$

$7.00 E+00$ 3.86E-01

$2.00 \mathrm{E}+01 \quad 1.98 \mathrm{E}-01$

01 1.22E-01

$6.34 \mathrm{E}+01$ 1.00E-02

$5.00 E+02 \quad 1.14 E-04$

7.00E+02 1.14E-04

$7.005+02$ 1.14E-04

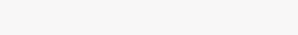




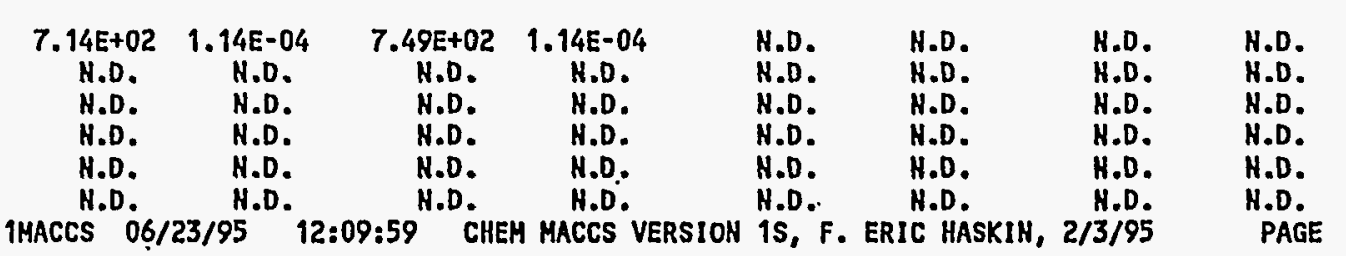

SOURCE TERH 1 OF 1 :

CHEM_HACCS EXAMPLE SOURCE TERM VX

RESULT NAME = EARLY FATALITY DISTANCE (KM)

ERL FAT/TOTAL RISK > 0.001

PEOPLE FRACTION =

OVERALL

$\begin{array}{cc}\text { X } & \text { PROB>=X } \\ 1.00 E-06 & 1.00 E+00 \\ 2.00 E-06 & 1.00 E+00 \\ 3.00 E-06 & 1.00 E+00 \\ 5.00 E-06 & 1.00 E+00 \\ 7.00 E-06 & 1.00 E+00 \\ 1.00 E-05 & 1.00 E+00 \\ 2.00 E-05 & 1.00 E+00 \\ 3.00 E-05 & 1.00 E+00 \\ 5.00 E-05 & 1.00 E+00 \\ 7.00 E-05 & 1.00 E+00 \\ 1.00 E-04 & 1.00 E+00 \\ 2.00 E-04 & 1.00 E+00 \\ 3.00 E-04 & 1.00 E+00 \\ 5.00 E-04 & 1.00 E+00 \\ 7.00 E-04 & 1.00 E+00 \\ 1.00 E-03 & 1.00 E+00 \\ 2.00 E-03 & 1.00 E+00 \\ 3.00 E-03 & 1.00 E+00 \\ 5.00 E-03 & 1.00 E+00 \\ 7.00 E-03 & 1.00 E+00 \\ 1.00 E-02 & 1.00 E+00 \\ 2.00 E-02 & 1.00 E+00 \\ 3.00 E-02 & 1.00 E+00 \\ 5.00 E-02 & 1.00 E+00 \\ 7.00 E-02 & 1.00 E+00 \\ 1.00 E-01 & 1.00 E+00 \\ 2.00 E-01 & 9.74 E-01 \\ 3.00 E-01 & 8.57 E-01 \\ 5.00 E-01 & 8.57 E-01\end{array}$

0.9500

EMER. RESP. \# 1 C. $\begin{array}{cc}X & \text { PROB> }=X \\ 1.00 E-06 & 1.00 E+00\end{array}$ $\begin{array}{ll}1.00 E-06 & 1.00 E+00 \\ 2.00 E-06 & 1.00 E+00\end{array}$ $\begin{array}{ll}2.00 E-06 & 1.00 E+00 \\ 3.00 E-06 & 1.00 E+00\end{array}$ 5.00E $=06 \quad 1.00 E+00$ 7.00E-06 1.00E +00 $1.00 E-05 \quad 1.00 E+00$ 2.00E-05 1.00E+00 3. $00 E-05 \quad 1.00 E+00$ $\begin{array}{ll}3.00 E-05 & 1.00 E+00\end{array}$ 7.00E-05
$1.00 \mathrm{O}-00 \mathrm{E}+00$ $\begin{array}{ll}\text { 7.0OE-05 } & 1.00 E+00 \\ 1.00 E-04 & 1.00 E+00\end{array}$ 1.00E-04 1.00E+00 2. $00 E-04 \quad 1.00 E+00$ 3.00E-04 $1.00 E+00$ 5. $00 E-04 \quad 1.00 E+00$ 7.00E-04 1.00E+00 1.00E-03 1.00E+00 2.00E-03 1.00E+00 $3.00 E-03 \quad 1.00 E+00$ 5.00E-03 1.00E+00 7.00E-03 1.00E+00 $1.00 \mathrm{E}-02 \quad 1.00 \mathrm{E}+00$ 2.00E-02 1.00E+00 3.00E-02 1.00E+00 $5.00 E-02 \quad 1.00 E+00$ 5.00E-02 1.00E+00 7.00E-02 1.00e+00 1.00E-O $1.00 \mathrm{E}+00$ 2.00E-01 8.57E-01 3.00E-01 8.57E-0 5.00E-01 8.57E-0

\subsection{0}

0.0000

EMER. RESP. \# 2 $X \quad P R O B>=X$ 1.00E-06 1.00E+00 .00E-06 1.00E+00 $3.00 E-06 \quad 1.00 E+00$ 5.00E-06 1.00E+00 7.00E-06 1.00E+00 1.00E-05 1.00E+00 . $00 E-05,1.00 E+00$ $3.00 E-05 \quad 1.00 E+00$ 5.00E-05 1.00E+00 7.00E-05 $1.00 E+00$ $1.00 \mathrm{E}-04 \quad 1.00 \mathrm{E}+00$ $2.00 E-04 \quad 1.00 E+00$ 3.00E-04 1.00E+00 $5.00 E-04 \quad 1.00 E+00$ 7.00E-04 1.00E+00 $1.00 E-03 \quad 1.00 E+00$ . :.00E-03 1.00E+00 $5.00 E-03 \quad 1.00 E+00$ 7.00E-03 1.00E+00 $1.00 E-02 \quad 1.00 E+00$ 2.00E-02 1.00E+00 3.00E-02 1.00E+00 5.00E-02 1.00E+00 7.00E-02 1.00E+00 . $00 E-01 \quad 1.00 E+00$ .00E-01 9.78E-0 3.00E-01 9.78E-0 5.00E-01 $9.78 \mathrm{E}-0$
EHER, RESP. \# 3

$X \quad P R O B>=X$

1.00E-06 1.00E+00 2.00E-06 $1.00 E+00$ 3.00E-06 $1.00 \mathrm{E}+00$ 5.00E-06 1.00E+00 7.00E-06 1.00E+00 1.00E-05 1.00E+00 2.00E-05 1.00E+00 3.00E-05 1.00E+00 5.00E-05 1.00E+00 7.00E-05 $\quad 1.00 \mathrm{E}+00$ $1.00 \mathrm{E}-04 \quad 1.00 \mathrm{E}+00$ 2.00E-04 1.00E+00 3.00E-04 1.00E+00 5.00E-04 $1.00 E+00$ 7.00E-04 1.00E+00 $1.00 E-03 \quad 1.00 E+00$ 2.00E-03 1.00E+00 3. $00 E-03 \quad 1.00 E+00$ 5.00E-03 1.00E+00 7.00E-03 1.00E+00 $1.00 E-02$ 1.00E+00 2.00E-02 1.00E+00 3.00E-02 1.0OE+00 7.00E-02 1.00E+00 1.00E-01 1.00E+00 2.00E-01 9.78E-01 $3.00 \mathrm{E}-01 \quad 9.78 \mathrm{E}-01$

5.00E-01 9.78E-0 


\begin{tabular}{|c|c|c|c|c|c|c|c|}
\hline $\begin{array}{c}7.00 E-01 \\
1.00 E+00 \\
2.00 E+00 \\
3.00 E+00 \\
5.00 E+00 \\
7.00 E+00 \\
1.00 E+01 \\
1.56 E+01 \\
\text { N.D. } \\
\text { N.D. } \\
\text { N.D. } \\
\text { H.D. } \\
\text { N.D. } \\
\text { N.D. } \\
\text { N.D. } \\
\text { H.D. } \\
\text { N.D. } \\
\text { ACCS 06 }\end{array}$ & $\begin{array}{c}\text { 3.89E-01 } \\
3.89 E-01 \\
3.26 E-01 \\
2.58 E-01 \\
1.55 E-01 \\
1.02 E-01 \\
8.54 E-02 \\
4.14 E-03 \\
\text { H.D. } \\
\text { N.D. } \\
\text { N.D. } \\
\text { N.D. } \\
\text { N.D. } \\
\text { N.D. } \\
\text { H.D. } \\
\text { N.D. } \\
\text { H.D. } \\
23 / 95 \quad 12\end{array}$ & $\begin{array}{c}7.00 E-01 \\
1.00 E+00 \\
2.00 E+00 \\
3.00 E+00 \\
5.00 E+00 \\
7.00 E+00 \\
1.00 E+01 \\
1.61 E+01 \\
\text { H.D. } \\
\text { H.D. } \\
\text { H.D. } \\
\text { H.D. } \\
\text { H.D. } \\
\text { H.D. } \\
\text { N.D. } \\
\text { H.D. } \\
\text { N.D. } \\
9: 59 \text { CHEF }\end{array}$ & $\begin{array}{c}3.89 E-01 \\
3.89 E-01 \\
3.26 E-01 \\
2.58 E-01 \\
1.55 E-01 \\
1.02 E-01 \\
8.54 E-02 \\
4.14 E-03 \\
\text { N.D. } \\
\text { N.D. } \\
\text { N.D. } \\
\text { N.D. } \\
\text { N.D. } \\
\text { N.D. } \\
\text { H.D. } \\
\text { H.D. } \\
\text { N.D. } \\
\text { MACCS VE }\end{array}$ & $\begin{array}{c}7.00 E-01 \\
1.00 E+00 \\
2.00 E+00 \\
3.00 E+00 \\
5.00 E+00 \\
7.00 E+00 \\
1.00 E+01 \\
1.13 E+01 \\
\text { N.D. } \\
\text { N.D. } \\
\text { N.D. } \\
\text { N.D. } \\
\text { N.D. } \\
\text { N.D. } \\
\text { N.D. } \\
\text { H.D. } \\
\text { N.D. } \\
\text { ION IS, F. }\end{array}$ & $\begin{array}{c}6.88 E-01 \\
6.88 E-01 \\
4.63 E-01 \\
3.61 E-01 \\
1.31 E-01 \\
3.62 E-02 \\
1.00 E-02 \\
1.00 E-02 \\
\text { N.D. } \\
\text { N.D. } \\
\text { N.D. } \\
\text { N.D. } \\
\text { N.D. } \\
\text { N.D. } \\
\text { N.D. } \\
\text { N.D. } \\
\text { N.D. } \\
\text { ERIC HASKI }\end{array}$ & $\begin{array}{c}7.00 E-01 \\
1.00 E+00 \\
2.00 E+00 \\
3.00 E+00 \\
5.00 E+00 \\
7.00 E+00 \\
\text { B.05E+00 } \\
\text { N.D. } \\
\text { N.D. } \\
\text { N.D. } \\
\text { N.D. } \\
\text { N.D. } \\
\text { N.D. } \\
\text { N.D. } \\
\text { N.D. } \\
\text { N.D. } \\
\text { N.D. } \\
\text { 2/3/95 }\end{array}$ & $\begin{array}{c}6.13 E-01 \\
6.13 E-01 \\
4.49 E-01 \\
2.74 E-01 \\
5.73 E-02 \\
1.05 E-02 \\
1.05 E-02 \\
\text { H.D. } \\
\text { H.D. } \\
\text { H.D. } \\
\text { H.D. } \\
\text { H.D. } \\
\text { H.D. } \\
\text { H.D. } \\
\text { H.D. } \\
\text { H.D. } \\
\text { H.D. }\end{array}$ \\
\hline
\end{tabular}

SOURCE TERM 1 OF $1:$

CHEM_MACCS EXAMPLE SOURCE TERM VX

RESULT NAME = POPULATION WEIGHTED RISK ERL FAT/TOTAL

$0-2.1 \mathrm{KM}$

$$
0.0500
$$

0.0000

EMER. RESP. \# 2 $X \quad$ PROB $>=X$ 1.00E-08 $1.00 E+00$ 2.00E-08 $1.00 E+00$ 3. $00 \mathrm{E}-08 \quad 1.00 \mathrm{E}+00$ $5.00 E-08 \quad 1.00 E+00$ 7.00E $1.00 \mathrm{C}$ $\cdot \begin{array}{ll}7.00 E-08 & 1.00 E+00 \\ 1.00 E-07 & 1.00 E+00\end{array}$ 2.00E-07 1.00E+00 3.00E-07 1.00E+00 5.00E-07 $1.00 E+00$ $7.00 E-07 \quad 1.00 E+00$ 1.00E-06 1.00E+00 2.00E-06 1.00E +00 $3.00 E-06 \quad 1.00 E+00$ $\begin{array}{ll}3.00 E-06 & 1.00 E+00 \\ 5.00 E-06 & 1.00 E+00\end{array}$ $\begin{array}{ll}5.00 E-06 & 1.00 E+00 \\ 7.00 E-06 & 1.00 E+00\end{array}$ $1.00 E-05 \quad 1.00 E+00$ 2. 00 E $-05 \quad 1.00 E+00$ 3.00E-05 1.00E+00
EMER. RESP. \# 3

$X \quad$ PROB $>=X$ 1.00E-08 1.00E+00 2.00E-0B $1.00 E+00$ 3.00E- 08 1.00E+ 00 5.00E-0B 1.00E+00 7.00E-08 1.00E+00 $1.00 E-07$ 1.00E+00 2.0OE-07 1.DOE+00 3.00E-07 1.00E+00 5.00E-07 1.00E+00 7.00E-07 1.00E+00 $1.00 \mathrm{E}-06 \quad 1.00 \mathrm{E}+00$ 2. $00 \mathrm{E}-06 \quad 1.00 \mathrm{E}+00$ 3.00E-06 1.00E+00 5.00E-06 1.00E+00 7.00E-06 $1.00 E+00$ $\begin{array}{ll}1.00 E-05 & 1.00 E+00 \\ 2.00 E-05 & 1.00 E+00\end{array}$ 3.00E-05 1.00E+00 


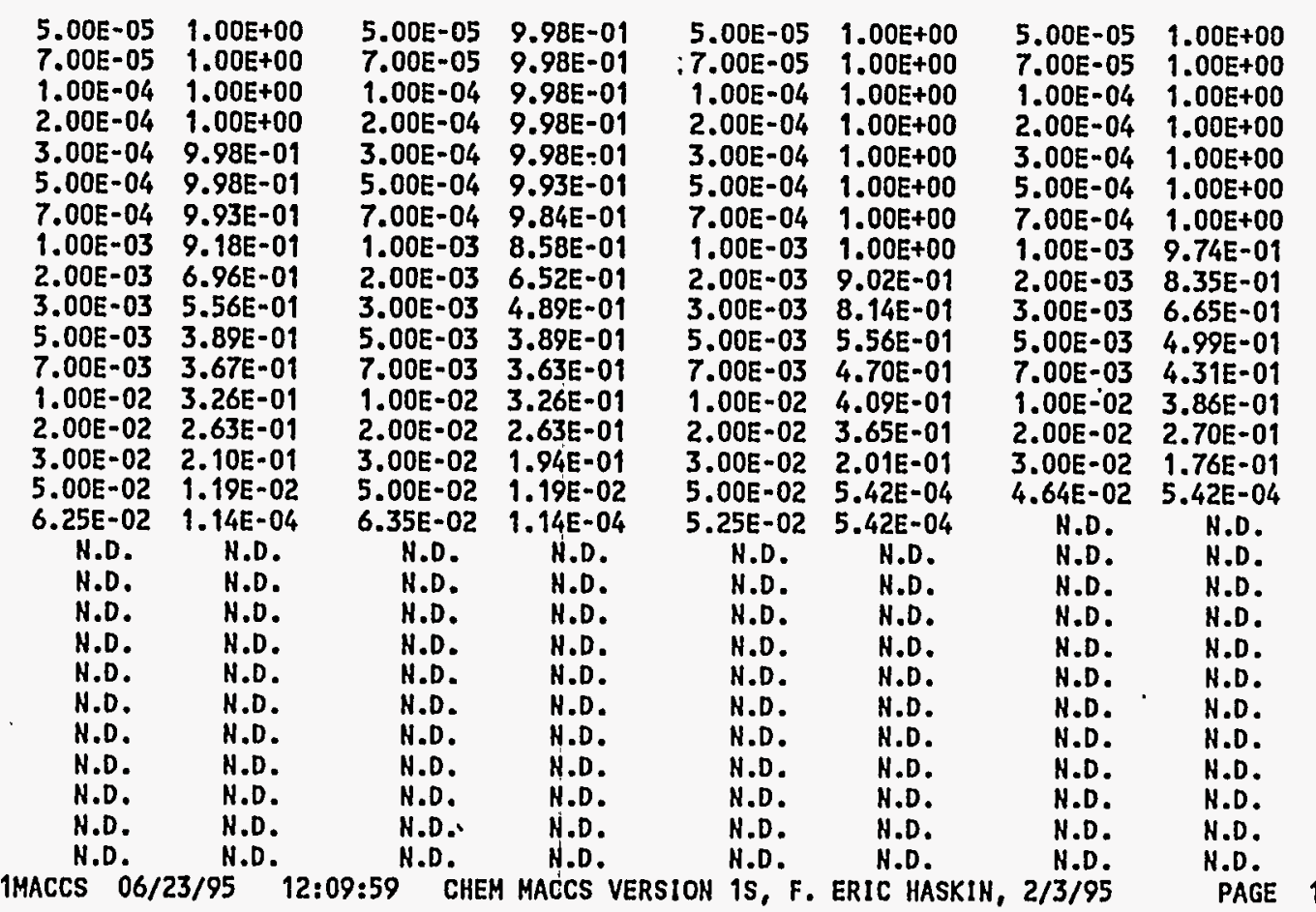

SOURCE TERH 1 OF 1 :

CHEM_HACCS EXAMPLE SOURCE TERM VX

RESULT HAME = POPULATION HEIGHTED RISK CAN INJ/TOTAL.

$0-16.1 \mathrm{KM}$

$$
\text { PEOPLE FRACTION = }
$$

$$
0.9500
$$

$$
0.0500
$$

0.0000

EMER. RESP. \# 2

$$
\begin{array}{cr}
X & \text { PROB }>=X \\
1.00 E-18 & 1.00 E+00
\end{array}
$$
$2.00 E-18$ 1. $1.00 E+00$ $3.00 E-18,1.00 E+00$ $5.00 E-18$ 1.0OE+0O $\begin{array}{ll}5.00 E-18 & 1.00 E+00 \\ 7.00 E-18 & 1.00 E+00\end{array}$ $\begin{array}{ll}7.00 E-18 & 1.00 E+00 \\ 1.00 E-17 & 1.00 E+00\end{array}$ $2.00 \mathrm{E}-17 \quad 1.00 \mathrm{E}+00$

EMER. RESP. \# 3

$\begin{array}{cc}X & \text { PROB }>=X \\ 1.00 E-18 & 1.00 E+00 \\ 2.00 E-18 & 1.00 E+00 \\ 3.00 E-18 & 1.00 E+00 \\ 5.00 E-18 & 1.00 E+00 \\ 7.00 E-18 & 1.00 E+00 \\ 1.00 E-17 & 1.00 E+00 \\ 2.00 E-17 & 1.00 E+00\end{array}$




\begin{tabular}{|c|c|c|c|}
\hline & $\begin{array}{c}1.00 E+00 \\
1.00 E+00 \\
1.00 E+00 \\
1.00 E+00 \\
1.00 E+00 \\
1.00 E+00 \\
1.00 E+00 \\
1.00 E+00 \\
1.00 E+00 \\
1.00 E+00 \\
1.00 E+00 \\
1.00 E+00 \\
1.00 E+00 \\
1.00 E+00 \\
1.00 E+00 \\
1.00 E+00 \\
9.73 E-01 \\
9.68 E-01 \\
9.44 E-01 \\
9.00 E-01 \\
7.95 E-01 \\
7.60 E-01 \\
6.88 E-01 \\
5.53 E-01 \\
3.26 E-01 \\
1.66 E-01 \\
1.20 E-01 \\
1.12 E-01 \\
1.07 E-01 \\
4.14 E-03 \\
N . D . \\
N . D . \\
N . D . \\
N .0 . \\
N . D \\
N . D . \\
N . D . \\
N . D . \\
N . D \\
N . D \\
N . D .\end{array}$ & $\begin{array}{l}3.00 E-17 \\
5.00 E-17 \\
7.00 E-17 \\
1.00 E-16 \\
2.00 E-16 \\
3.00 E-16 \\
5.00 E-16 \\
7.00 E-16 \\
1.00 E-15 \\
2.00 E-15 \\
3.00 E-15 \\
5.00 E-15 \\
7.00 E-15 \\
1.00 E-14 \\
2.00 E-14 \\
3.00 E-14 \\
5.00 E-14 \\
7.00 E-14 \\
1.00 E-13 \\
2.00 E-13 \\
3.00 E-13 \\
5.00 E-13 \\
7.00 E-13 \\
1.00 E-12 \\
2.00 E-12 \\
3.00 E-12 \\
5.00 E-12 \\
7.00 E-12 \\
1.00 E-11 \\
2.00 E-11 \\
2.04 E-11 \\
N . D . \\
N . D . \\
N . D . \\
N . D . \\
N . D . \\
N . D . \\
N . D . \\
N . D .\end{array}$ & $\begin{array}{l}1.00 \mathrm{E}+0 \\
1.00 \mathrm{E}+0 \\
1.00 \mathrm{E}+0 \\
9.59 \mathrm{E}-0 \\
9.59 \mathrm{E}-0 \\
9.59 \mathrm{E}-0 \\
9.59 \mathrm{E}-0 \\
9.37 \mathrm{E}-0 \\
9.37 \mathrm{E}-0 \\
9.30 \mathrm{E}-0 \\
9.28 \mathrm{E}-0 \\
9.25 \mathrm{E}-0 \\
9.22 \mathrm{E}-0 \\
9.18 \mathrm{E}-0 \\
9.18 \mathrm{E}-0 \\
9.08 \mathrm{E}-0 \\
8.89 \mathrm{E}-0 \\
8.89 \mathrm{E}-0 \\
8.68 \mathrm{E}-0 \\
7.83 \mathrm{E}-0 \\
7.51 \mathrm{E}-0 \\
6.83 \mathrm{E}-0 \\
5.37 \mathrm{E}-0 \\
3.26 \mathrm{E}-0 \\
1.60 \mathrm{E}-0 \\
1.20 \mathrm{E}-0 \\
1.12 \mathrm{E}-0\end{array}$ \\
\hline
\end{tabular}

$3.00 E-17 \quad 1.00 E+00$ $5.00 E-17 \quad 1.00 E+00$ $7.00 E-17 \quad 1.00 E+00$ $1.00 E-16 \quad 1.00 E+00$ 2.00E-16 $1.00 E+00$ $3.00 E-16 \quad 1.00 E+00$ $5.00 E-16 \quad 1.00 E+00$ \begin{tabular}{l}
$7.00 E-16 \quad 1.00 E+00$ \\
\hline
\end{tabular} $1.00 \mathrm{E}-15 \quad 1.00 \mathrm{E}+00$ 2.00E-15 $1.00 \mathrm{E}+00$ 3. $00 \mathrm{E}-15$ 1.00E+00 $\begin{array}{ll}3.00 E-15 & 1.00 E+00 \\ 5.00 E-15 & 1.00 E+00\end{array}$ $\begin{array}{ll}5.00 E-15 & 1.00 E+00 \\ 7.00 E-15 & 1.00 E+00\end{array}$ $1.00 E-14 \quad 1.00 E+00$ $2.00 E-14 \quad 1.00 E+00$ $3.00 E-14 \quad 1.00 E+00$ $5.00 E-14 \quad 1.00 E+00$ $7.00 E-14 \quad 1.00 E+00$ $1.00 \mathrm{E}-13$ 1. $1.00 \mathrm{E}+00$ $2.00 \mathrm{E}-13 \quad 1.00 \mathrm{E}+00$ $\begin{array}{ll}2.00 E-13 & 1.00 E+00 \\ 3.00 E-13 & 1.00 E+00\end{array}$ 5.00E-13 9.24E-01 7.00E-13 8.72E-01 1.00E-12 7.34E-01 2.00E-12 4.44E-01 $3.00 E-12 \quad 3.20 E-01$ 5.00E-12 1.04E-01 5.00E-12 1.04E-01 $9.89 E-12 \quad 1.00 E-02$ $\begin{array}{cc}9.89 E-12 & 1.00 E-02 \\ \text { N.D. } & \text { H.D. }\end{array}$ N.D. N.D. N.D. N.D. N.D. N.D.

N.D. N.D.

N.D. N.D. N.D. N.D. N.D. N.D. N.D.' N.D.
$3.00 E-17 \quad 1.00 E+00$ $5.00 E-17 \quad 1.00 E+00$ $7.00 E-17 \quad 1.00 E+00$ $1.00 \mathrm{E}-16 \cdot 1.00 \mathrm{E}+00$ 2.00E-16 1.00E+00 $3.00 E-16 \quad 1.00 E+00$ 5.00E-16 1.00E+00 7.00E-16 1.00E+00 $1.00 E-15,9.00 E+00$ $2.00 E-15,00 E+00$ $2.00 E-15$ 1.00E+00 3.00E-15 1.00E+00 5.00E-15 1.00E+00 $7.00 \mathrm{E}-15 \quad 1.00 \mathrm{E}+00$ $1.00 \mathrm{E}-14 \quad 1.00 \mathrm{E}+00$ 2.00E-14 1.00E+00 $3.00 \mathrm{E}-14 \quad 1.00 \mathrm{E}+00$ 5.00E-14 1.00E+00 7.00E-14 1.00E+00 $1.00 E-13 \quad 1.00 E+00$ 2. $00 E-13 \quad 1.00 E+00$ 3.00E-13 9.47E-01 5.00E-13 8.28E-01 7.00E-13 7.30E-01 1.00E-12 5.80E-01 2. $00 \mathrm{E}-12$ 3.24E-01 3.00E-12 1.51E-01 5. 00E-12 1.09E-02 5. $88 E-12$ $7.09 E-02$ N.D. N.D. N.D. N.D. N.D. N.D. H.D. H.D. N.D. N.D.

N.D. N.D.

N.D. N.D.

N.D. N.D

N.D. N.D

SUCCESSFUL COMPLETION OF MACCS HAS ACHIEVED

THIS JOB REQUIRED A TOTAL OF 350.918 CPU SECONDS

INPUT PROCESSING REQUIRED $4.668 \mathrm{CPU}$ SECONDS SIMULATIOU REQUIPED $323.684 \mathrm{CPU}$ SECONDS

OUTPUT PROCESSING REQUIRED 22.566 CPU SECONDS 


\section{E.1 CHEH_MACCS INPUT/OUTOUT LISTIKG FOR COHPARISOH}

MACCS 07/12/95 22:04:59 CHEM MACCS VERSION 1S, F. ERIC HASKIN, 2/3/95

P1: ATMOS USER INPUT (UNIT 24) $=$ D2PC 1 -INP

P2: EARLY USER INPUT (UNIT 25) $=$ D2PC_2.IHP

P3: CHRONC USER INPUT (UNIT 26) $=$ "II

DOSE FACTORS (UNIT 27) $=$ DOSDATA.INP

P4: METEOROLOGY DATA (UNIT 28) $=$ "'!

$\begin{array}{ll}\text { P5: } & \text { SITE DATA INPUT (UNIT 29) }=\text { III } \\ \text { P6: } & \text { LIST OUTPUT (UNIT O6) }=\text { D2PC.OUT }\end{array}$

USER INPUT IS READ FROM UNIT 24

RECORD IDENTIFIER FIELDS 11 CHARACTERS LONG ARE EXPECTED.

THE FIRST 100 COLUHMS OF EACH INPUT RECORD ARE PROCESSED.

THE MAXIMUM NUMBER OF IDENTIFIER RECORDS THAT HAY BE SAVED AS THE BASE CASE IS 1000.

RECORD

NUHBER

RECORD

* GENERAL DESCRIPTIVE TITLE DESCRIBING THIS "ATMOS" INPUT

1 RIATNAM1001 'CHEM MACCS run for comparison, ATMOS INPUT'

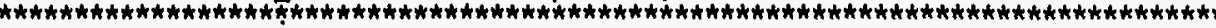
* gEOMETRY DATA BLOCK, LOADED BY INPGEO, STORED IN /GEOH/ * number of radial spatial elements GENUMRADO01 16

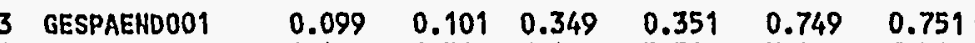

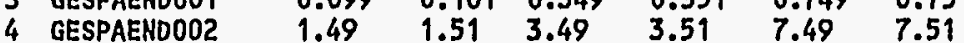

5 GESPAENDOO3 14.8 GESPAENDO03 $14.9 \quad 15.1 \quad 34.9 \quad 35.1$ * CHEMICAl SPECIES DATA blOCK, LOADED BY INPISO, STORED IN /ISONAM/

* number of chemical species

6 ISHUHISOOOI 1

* chenical species data

* chenical species data

* CHEHICAL SPECIES WETDEP DRYDEP SKIN DEP.

ISOTPGRP001 GA-LIQ .FALSE. FALSE. 0.

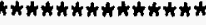

* WET DEPOSITION DATA BLOCK, LOADED BY INPWET, STORED IN /WETCON/ 
8 HDCHASHYOO1 9.5E-5 (JON HELTON AFTER JONES, 1986) * hashout coefficient number tho. exponential factor

9 HDCHASH2O01 0.8 (JON HELTON AFTER JONES, 1986)

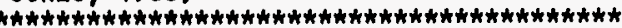
* DRY DEPOSITION DATA BLOCK, LOADED BY INPDRY, STORED IN /DRYCON/

* number of particle size groups

10 DDNPSGRPO01 1

* Deposition velocity of each particle size group (M/S) *

11 DDVDEPOS001 0.001

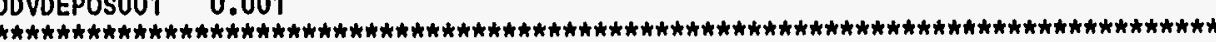
* DISPERSION PARAMETER DATÁ BLOCK, LOADED BY INPDIS, STORED IN /OISPY/, /DISPZ/

* SIGMA $=A X * *$ B hHERE A AND 8 VALUES ARE FROM TADMOR AND GUR (1969)

* linear term of the expression for sigma-y, 6 stability classes

* Stability class: A B C $\quad$ D $\quad$ E

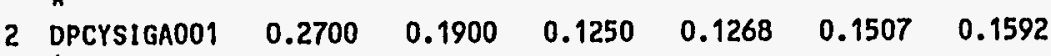

* exponential tern of the expression fOR SIGMA-Y, 6 Stability Classes

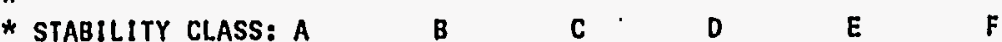

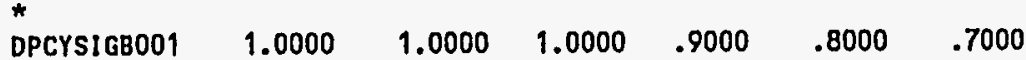

* LINEAR TERH OF THE EXPRESSION fOR SIGMA-Z, 6 STABILITY CLASSES

* stabillitr class: A B C , D

* STabillity class: a

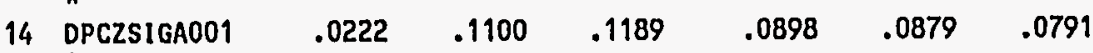

* EXPONENTIAL TERH OF THE EXPRESSION FOR SIGMA-Z, 6 STABILITY CLASSES

* stabillity class; A B C D

$\begin{array}{llllllll} & \text { DPCZSIGBO01 } & 1.400 & 1.0000 & .9000 & .8500 & .8000 & .7500\end{array}$

* LIHEar scaling factor for sIGMa-y function, nORMally 1 


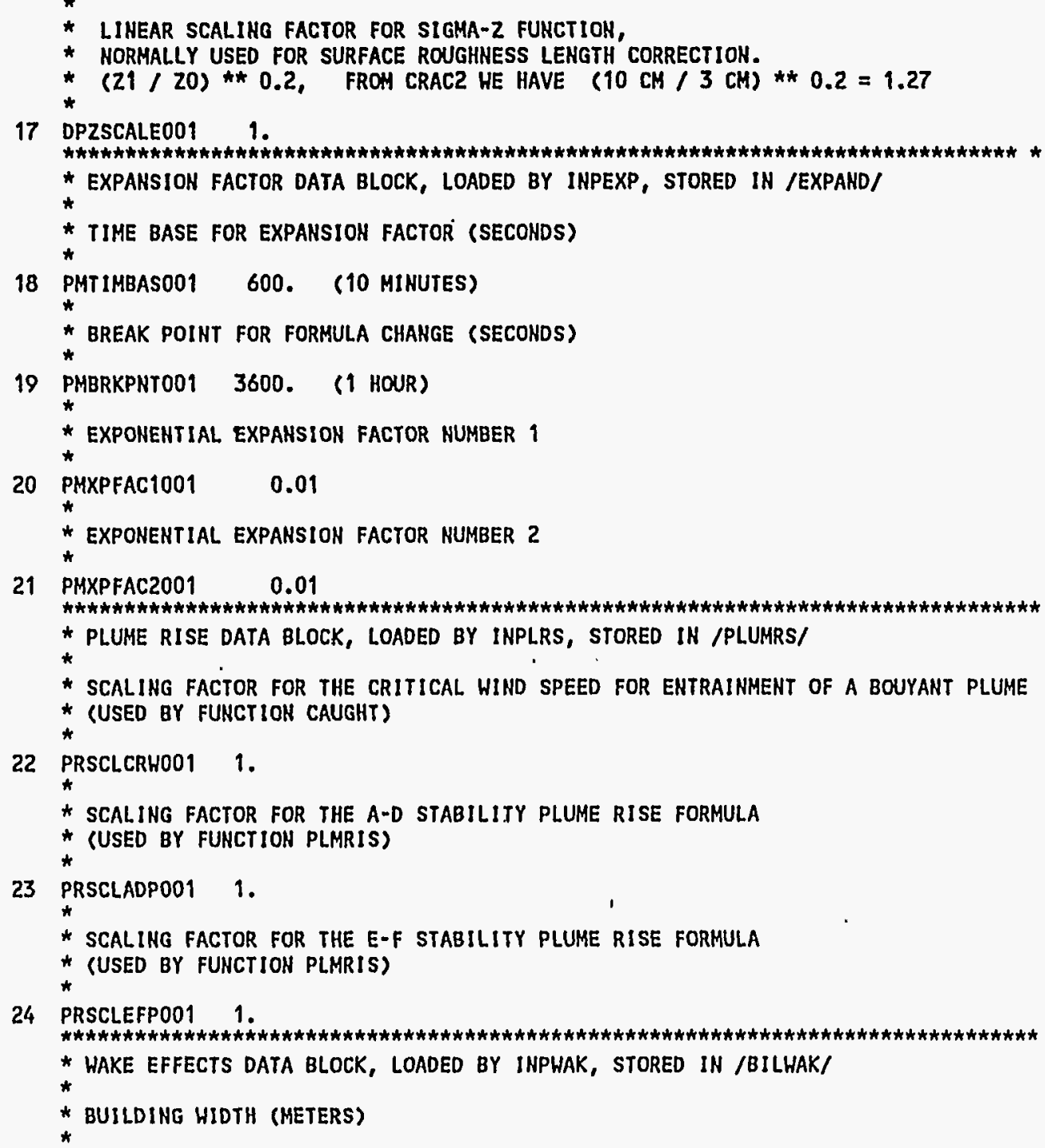


25 HEBUILDHOO1 i. * SURRY<smiles>[131IH]</smiles>

* BUILDING HEIGHT (METERS)

26 HEQUILDH001 1. * SURRY

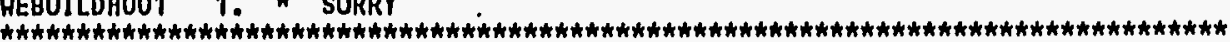

* Release data block, loaded by inPrel, stored in /atham2/, /MUlRel/

*

27 RDATNAM2001 'CHEM_MACCS GA EXAMPLE PROBLEM, SOURCE TERM'

* time after accident initiation hHEN the accident reaches general emergency

* CONDITIONS (AS Defined in NUREg-0654), OR hHEH PLANT PERSONNEL. CAN RELIABLY

* PREDict THAT GEMERAL. EMERGENCY CONDITIONS HILL BE ATTAINED

28 RDOALARMOO1 1300 .

* number of plume segments that are released

29 RDNUMRELOO

* Selection of Risk dominant plume

30 RDMAXRIS001 1

* reference time for dispersion

31

ODRETIMO01 0.00 V

* heat content of the release segments (H)

* A VALUE SPECIFIED FOR EACH OF THE RELEASE SEgMEHTS

32 RDPLHEATOO

3.35

* HEIGHT OF THE PLUME SEGMENTS AT RELEASE (M)

* A VALUE SPECIFIED For EACH OF tHe RELEASE SEgMents

33 RDPLHITEOOI

0.

* DURATION of the pluME SEgMENTS (S)

* A VALUE SPECIFIEd FOR EACH OF THE RELEASE SEGMEHTS

34

3600

* time of release for each pluMe

* a VAlUE SPECIFIEd for EACH OF the Release segments

35 RDPDELAYOO1 0. 
* particle size distribution of each chemical species

* THE fRACTIONS fOR EACH CHEMICAL SPECIES (ROH) MUST SUM tO ONE.

*

36 RDPSDISTOO1

$0.001 \mathrm{~m} / \mathrm{s}$

* inventory of each chemical species subject to release

* CHEhICAL QUANTITY

SPECIES RELEASED

(kg)

37 RDCORIHVOOI GA-LIQ 1.22

* scaling factor to adjust the Inventory

38 RDCORSCA001 1.000

*

* Release fractions for chemical species by plume

*

* PLUME: GA-LIQ

39 RDRELFRCOO1 1.0

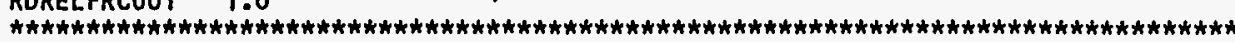

* OUTPUT CONTROL DATA BLOCK, LOADED BY INPOPT, STORED IN /STOPME/, /ATMOPT/

* Flag to indicate that this is the last program in the series to be run

40 OCENDAT 1001 .FALSE. (SET THIS VALUE TO .TRUE. TO SKIP EARLY AND CHRONC)

OCIDEBUG001 0

* name of the chemical species to be listed on the dispersion listings

*

*OCNUCOUTOO1 VX-VAP

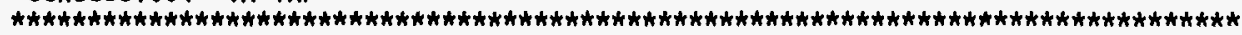

* Meteorological sampling data block

*

* meteorological sampling option code:

* METCOD $=1$, USER SPECIFIED DAY AND hOUR IN THE yEAR (FROM MET FILE),

* 2, heATHER CATEgory bin SAMPLing,

3. 120 HOURS OF WEATHER SPECIFIED ON THE ATHOS USER INPUT FILE,

* 4, CONSTANT MET (BOUNDARY hEATHER USED FRON THE START)

M1METCOD001 4 (BOUNDARY WEATHER USED FROM THE START) 
43 M1HRINITOOI $1.0 \quad 24.0$

* last spatial interval for measured heather

* Last spatial in

44 M2LIMSPAOO1 8

* boundary heather hixing layer height

*

45 M2BNDHXHOO1 220. (METERS)

* boundary heather stability class index

46 M2IBDSTBOO1 1 (D-STABILITY)

* Boundary heather rain rate

47 M2BNDRAMOO1 0. (MM/HR)

*

* BOUNDARY heather hINo SPEEd

48 MZBHDHNDOO1 2. (M/S)

* start day in the year for the single heather sequence

*

49 H3ISTRDYO01 157 (START TIME FOR PEAK ECONONIC COST OF SAMPLE PROBLEM A)

* start hour in the day for the single heather sequence

* START hOUR IN THE DAY FOR the SINGLE heATHER SEQUENCE

50 M3ISTRHRO01 10 (START TIME FOR PEAK ECONOMIC COST OF SAMPLE PROBLEM A)

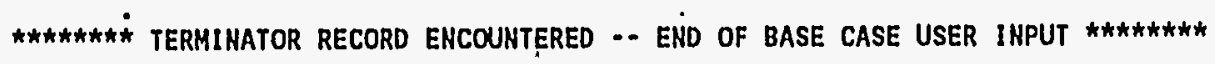

USER INPUT PROCESSING SUMMARY - BASE CASE

NUMBER OF RECORDS READ

NUMBER OF BLANK OR COMMENT RECORDS READ $=204$

NUMBER OF TERMINATOR RECORDS

NUMBER OF RECORDS PROCESSED

NUMBER OF PROCESSED RECORDS DUPLICATED

NUMBER OF PROCESSED RECORDS SORTED

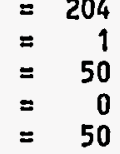


1 RELEASED INVENTORY OF ALL PLUMES

GA-LIQ

$1.22 E+00$

USER INPUT IS READ FRON UNIT 25

RECORD IDENTIFIER FIELDS 11 CHARACTERS LONG ARE EXPECTED.

THE FIRST 100 COLUMNS OF EACH INPUT RECORD ARE PROCESSED.

THE MAXIMUM NUMBER OF IDENTIFIER RECORDS THAT MAY BE SAVED AS THE BASE CASE IS 1000.

RECORD

NUMBER

RECORD

* general descriptive title describing this "Early" input file

1 MIEANAM1001 'D2PC_2.INP, CHEM_MACCS EXAMPLE PROBLEM GA_A, EARLY INPUT'

* DISPERSion moDel option cODE: 1 * straight liNe

* 2 * Hind-ShiFt hith rotation

*

3 * HIND-SHIFT HITHOUT ROTATION

2 milplume001 1 (STRAight LINE PLUME)

* nUMBer of fine grid subdivisions USED by the model

3 MinUMFINOO1 7 (3, 5 OR 7 ALLOHED $)$

* LeVel of debug outPUt required, normal RUNS shoUld speCify zero

4 MIIPRINTOO1 0 (TURN OFF THE DEBUG PRINT)

* logical flag signifying that the breakdown of risk by heather category

* bin are to be presented to shoh their relative contribution to the mean

* RISBIN

MIRISCATOO1 FALSE.

*

* flag indicating if hind-roses froh athos are to be overridden

6 MIOVRRTO0O1. FALSE. (USE THE WIHD ROSE CALCULATED FOR EACH WEATHER BIN)

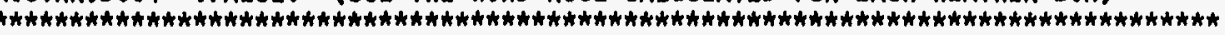

* POPULATION DISTRIBUTION DATA BLOCK, LOADED BY INPOPU, STORED IN /POPDAT/

7 PDPOPFLGOO1 UNIFORH

8 PDIBEGINOO1 1 (SPATIAL INTERVAL AT WHICH POPULATION BEGINS)

9 PDPOPDENOO1 50. (POPULATION DENSITY (PEOPLE PER SQUARE KILOMETER))

D

* DOSE DEFINITION DATA BLOCK, LOADED BY INORGA, STORED IN /EARDIM/ ANO /ORGNAM/ 
ODNUMORGOOI 3

*

11 ODORGMAMOO1

13

$\begin{array}{llll}\text { ODORGNAMO02 'LSK4' 'SKN ACU' } & \text { 'GA' } & 1.0 \\ \text { ODORGNAMOO3 'VCOD' IINH LIF' } & \text { 'GA' } & 1.0\end{array}$

* SHIELDING AND EXPOSURE FACTORS, LOADED BY INDFAC, STORED IN /EADFAC/

* three values of each protection factor are supplied,

* ONE FOR EACH TYPE OF ACTIVTY:

\section{* ACTIVITY TYPE:}

* 1 - EVACUEES WHILE MOVING

2 - MORHAL ACTIVITY IH SHELTERING AND EVACUATION ZONE

3 - SHELTERED ACTIVITY

* Protection factor for inhalation

*

14
ROTIN001
1.
1.
0.33 * VALUES FOR NORMAL ACTIVITY AND

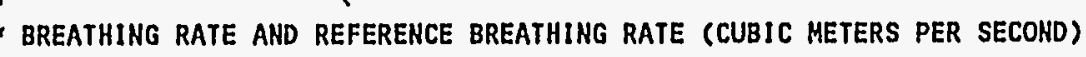

15 SEBRRATE001 2.66E-4 2.66E-4 2.66E-4 * BREATHING RATE

16 SEBRRATEOD2 2.66E-4 2.66E-4 2.66E-4 * REFERENCE BREATHING RATE

* SKIN PROTECTION FACTOR

* VALUES FOR NORMAL ACTIVITY AND SHELTERING SELECTED BY NRC STAFF

17 SESKPFACO01 $1.0 \quad 1$. 0.33 * FOR LIQUID

18 SESKPFACOO2 1.0 1. 0.33 * FOR VAPOR

* RESUSPENSION INHALATION MODEL CONCENTRATION COEFFICIENT (/METER)

RESCON $=1, E-4$ IS APPROPRIATE FOR MECHANICAL RESUSPENSION BY VEHICLES

RESHAF $=2.11$ DAYS CAUSES 1.E-4 TO DECAY IN ONE HEEK TO 1.E-5, THE VALUE

OF RESCON USED IN THE FIRST TERH OF THE LONG-TERM RESUSPENSION EQUATION

OF RESCON USED
USED IN CHRONC.

19 SERESCONOO1 1.E-4 (RESUSPENSION IS TURNED ON)

* RESUSPENSION CONCENTRATION COEFFICIENT HALF-LIFE (SEC) 
20 SERESHAFO01 $1.82 E 5$ (2.11 DAYS)

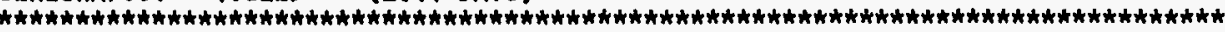

* EVACUATION ZONE DATA BLOCK, LOADED BY EVNETH, STORED IN /METHOR/, /EOPTIO/

* SPECIFIC DESCRIPTION OF THE EṀERgEnCY RESPONSE SCENARIO BEING USED

*

21 ezeanamzoo1 'no evacuation '

* THE TYPE OF WEIGHTING tO BE APPLIED tO THE EMERGENCY RESPONSE SCENARIOS

* YOU MUST SUPPLY A VALUE OF 'TIME' OR 'PEOPLE'

22 EZHTHAMEO01 'PEOPLE'

* heighting fraction applicable to this scenario

23 EZHTFRACO01 1.

* Last Ring IN the movement zone

24 EZLASMOVO01 0 (A ZERO TURNS OFF THE EVACUATION MODEL)

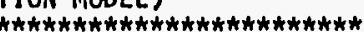

* shelter and Relocation zone dATA block, loAded by INPEMR,

*

* time to take shelter in the Inher shelter zone (seconds frow oalarm)

25 SRTTOSH1001 0 . '(THERE IS NO INNER SHELTER ZONE)

* Shelter duration IN the INNER Shelter zoNe (SEConds from taking shelter)

SRSHELT1001 0 . (THERE IS NO INNER SHELTER 2ONE)

* Last ring of the outer shel.Ter zONE

27 SRLASHE2001 0 (THERE IS NO OUTER SHELTER ZONE)

* tIME to take slielter In the oUter shelter zONE (SECONDS from OALARH)

* tIME to take sielter in the outer shelter zone (SEconds

28 SRTTOSHzOO1 0 (THERE IS NO OUTER SHELTER ZONE)

* Shelter duration in the outer shelter zone (SEconds fron taking shelter)

29 SRSHELT2001 0. (THERE IS NO OUTER SHELTER ZONE)

* duRATION of the emergency phase (SECONDS from PLUME arRival.)

* DURATION OF THE EMERGENCY PHASE (SECONDS FROH PLUME ARRIVAL)

30 SRENDEMP001 604800. (ONE HEEK) 
* critical dose for relocation decisions

31 SRCRIORGO01 'LSK3'

* hot spot relocation time (SECÓNDS from plume arRival)

*

32 SRTIMHOTO01 43200. (ONE-HALF DAY)

* Normal relocation time (SECONDS fron plume arRival)

33 SRTIMNRMO01 86400. (ONE DAY)

* hot SPOT RELOCATION DOSE CRITERION THRESHOLd (SIEVERTS)

34 SRDOSHOTO01 0.5 (50 REM DOSE TO WHOLE BOOY IN 1 HEEK TRIGGERS RELOCATION) * NORMal RElocation dose CRITERION THREshold

35 SRDOSNRMO01 0.045 (25 MG/M^3-S VAP AIR CONC DOSE IN 1 HK TRIGGERS RELOCATIOH)

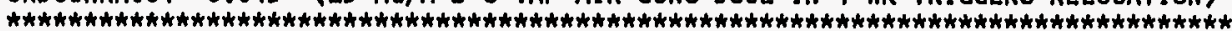
* EARLY FATALITY MODEL PARAMETERS, LOADED BY IHEFAT, STORED IN /EFATAL/ * number of early fatality effects

36 EFNUMEFAO01 2

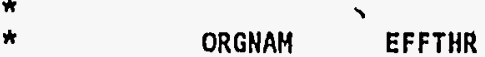

37 EFATAGRPOO1 LSK3

8 EFATAGRPO02 'LSK4

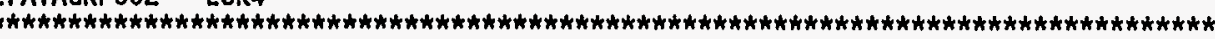
* EARLY INJURY MODEL. PARAMETERS, LOADED BY INEINJ, STORED IN /EINJUR/

* NUMber of early INJURY efFEcts

39 EINUMEINOOI 1
*
EINAME
ORGNAM
EISUSC EITHRE

40 EINJUGRP001 'CLP, PRL, CONVL' 'LSK3'

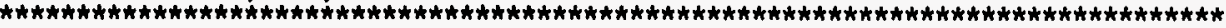

* ACUTE EXPOSURE CANCER PARAMETERS, LOADED by INACAN STORED IN /ACANCR/.

* number of acute exposure cancer effects

41 LCNUMACAOO1 1 
* THRESHOLO DOSE FOR APPLYING DDREFA

42 LCDDTHRE001 0.

* dose threshold for limear dose Response

LCACTHRE001 0.

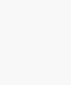

* aCNAME ORgnam acsusc dosefa dosefb cFrisK cirisk dDREFa

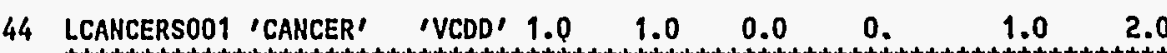

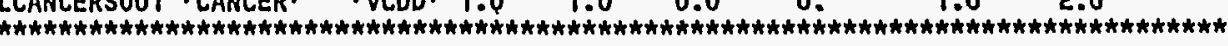

* RESULT 1 OPTIONS BLOCK, LOADED BY INOUT1, STORED IN /INOUT1/

* total number of a given efFect (LATENt cancer, early deAth, early inJury)

* Number of desired Results of this type

45 TYPEINUMBER

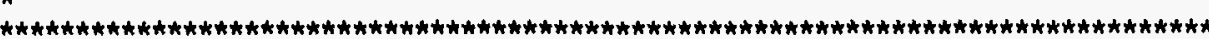

* RESULT 2 OPTIONS BLOCK, LOADED BY INOUT2, STORED IN /INOUT2/

* FURTHEST DISTANCE AT WHICH A GIVEN RISK OF EARLY DEATH IS EXCEEDED.

* NUMBER of DESIREd RESULTS OF THIS TYPE

46 TYPEZNUMBER 0

*

* RESULT 3 OPTIONS BLOCK, LOADED BY INOUT3, STORED IN /INOUT3/

* NUMBER OF PEOPLE HHOSE DOSE EXCEEDS A GIVEN THRESHOLD.

* number of desired results of this type

47 TYPE3NUMBER 0

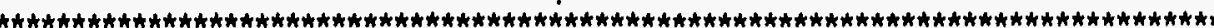

* RESULT 4 OPTIONS BLOCK, LOADED BY INOUT4, STORED IN /INOUT4/

* 360 DEGREe AVERAGE RISK OF a GIVEN EFFECT AT A GIVEN DISTANCE.

* POSSIBLE TYPes of EFFECTS ARE:

'erl fat/total'

'ERL INJ/INJURY NAME'

'CAN FAT/CANCER NAME'

'CAN FAT/TOTAL'

* number of desired results of this type 
53 TYPEBHUMBER 0

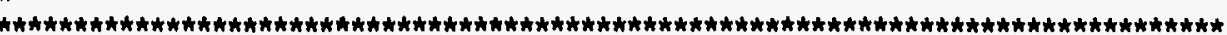

* RESUlT 9 OPTIONS BLOCK, LOADED BY INOUT9, STORED IN /INOUT9/

* FURTHEST distahCE at hHICH a GIVEN RISK Of EARLY INJURY IS EXCEEDED.

* number of desired results of this type

54 TYPESNUMBER 0

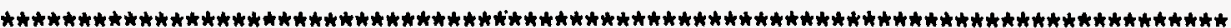

* RESULT 10 OPTIONS BLOCK, LOADED BY INOUTO, STORED IN /INOUTO/

* AREA IN WHICH GROUND CONCENTRATION OF A GIVEN AGENT EXCEEDS A GIVEN THRESHOLD.

* nUMber of desired resUlts of thIS tYPe

5 TYPEONUMBER 0

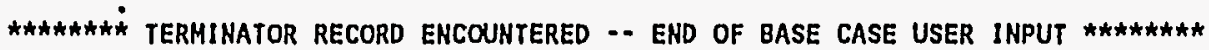

USER INPUT PROCESSING SUMMARY - BASE CASE

NUMBER OF RECORDS READ

NUMBER OF BLANK OR COMMENT RECORDS READ

NUMBER OF TERMINATOR RECORDS

UUMBE OF TERHINATOR RECORDS

NUMBER OF PROCESSED RECORDS DUPLICATED

NUMBER OF PROCESSED RECORDS SORTED

NO EVACUATION REQUESTED

CALCULATING A UNIFORM POPULATION DISTRIBUTION

READING FROM A DOSE CONVERSION FILE HITH THE FOLLOWING HEADER:

CHEM_MACCS File DOSDATA.INP: Changed bY E. HASKIN $10 A P R 95$

Dose conversion factors for CHEM MACCS Version 1.0

1 THIS PROGRAM CURRENTLY ALLOWS THE GENERATION OF UP TO 394 RESULTS

YOU HAVE REQUESTED 16 RESULTS FROM "EARLY" COMPOSED OF:

0 RESULTS OF TYPE

0 RESULTS OF TYPE 
0 RESULTS OF TYPE 3

0 RESULTS OF TYPE 4

0 RESULTS OF TYPE 5

16 RESULTS OF TYPE 6

O RESULTS OF TYPE 7

0 RESULTS OF TYPE 8

O RESULTS OF TYPE 9

0 RESULTS OF TYPE 10

1 TRIAL DAY

HOUR BIN PRBMET
1
157
10
O $1.00 \mathrm{E}+00$

1 DATE AND TIME OF RUN = MACCS 07/12/95 22:04:59 CHEM MACCS VERSION 1S, F. ERIC HASKIN, 2/3/95

"ATMOS" DESCRIPTION = CHEM_MACCS run for comparison, ATHOS INPUT

"EARLY" DESCRIPTION = D2PCZ2. INP, CHEM MACCS EXAMPLE PROBLEM GA A, EARLY INPUT

SOURCE TERM 1 OF 1 :

CHEM_MACCS GA EXAMPLE PROBLEH, SOURCE TERM

RESULTS FOR A SINGLE EMERGENCY RESPONSE COHORT HITHOUT ANY HEIGHTING FRACTIONS BEING APPLIED

COHORT 1 = NO EVACUATION

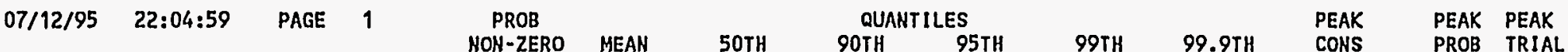

CENTERLINE DOSE AT SOME DISTANCES (mg-mi

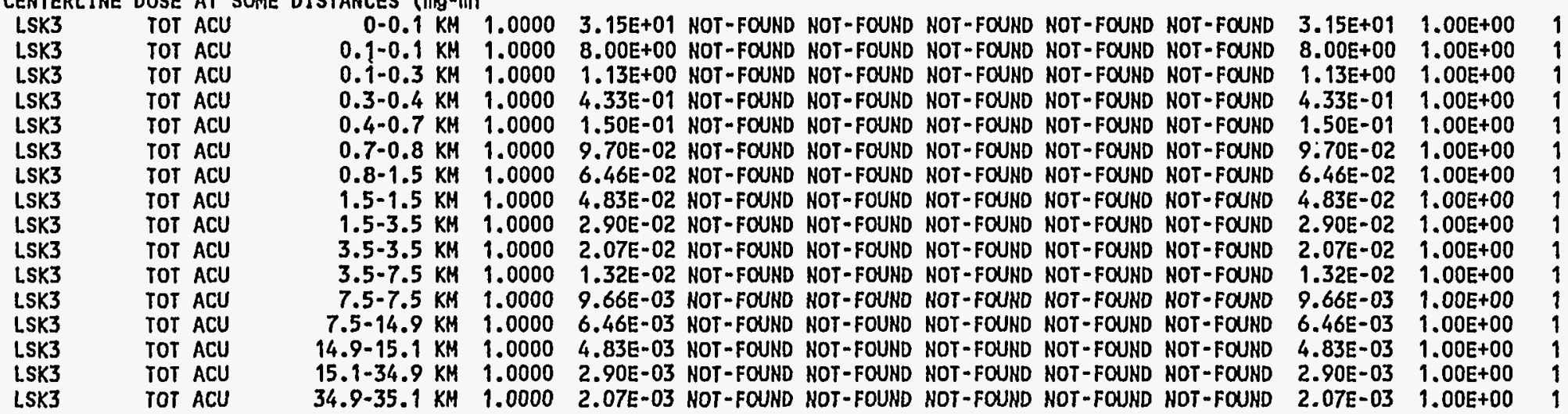

SUCCESSFUL COMPLETION OF MACCS HAS ACHIEVED

THIS JOB REQUIRED A TOTAL OF 2.586 CPU SECONDS

INPUT PROCESSING REQUIRED

SIMULATION REQUIRED

$2.313 \mathrm{CPU}$ SECONDS

CPU SECONDS

0.055 CPU SECONDS 


\section{E.2 D2PC IMPUT/OUTPUT LISTIKG FOR COAPARISOH}

$$
\text { [ DOHNHIND HAZARD PROGRAM DZPC | }
$$

TYPE ? FOR DEFINITIOHS

1. YOUR NOVICE LEVEL: $3,2,1$ OR 0 NOV

INPUT:

2. LOCATION LOC

$A A D, D P G, E H A, J H I, L B G, H A P, P B A, P A D, R H A, U A D, E U R, N D F$

INPUT:

4. HEIGHT OF MIXING LAYER HML

INPUT:

5. MUNITION TYPE

MUN $105,155,81 \mathrm{IN}, 500,750, M 55,525,139, M 23,4.2$, NON

INPUT:

6. AGENT TYPE AGN $G A, G B, G D, G F, V X, B Z, H Y, U D, H D, H 1, H 3, H T, L L, A C$, INPUT: $C G, C K, D H, E G, Q L, D F, D C, T C, P R, I P, Z S, K B, N A$
8. RELEASE TYPE
REL

INPUT: INS , EVP, SEM, VAR, STK, STJ, FLS, FIR, IGL, EVS

9. STABILITY TYPE $A, B, C, D, E, F, U, S, H$

INPUT:

10. WINDSPEED (M/SEC)

STB

INPUT:

HND

13. $Q()(M G), T Q()(H I N)$

INPUT:ALL OTHER INPUT

INPUT: DIC)S (ASCENDING)

ALL OTHER INPUT

1 MUN:NON AGN:NA REL:SEM HND $=2.0(M / S)$ TMP $=.0(C)$ NDF- STB:A

Q(MG) TS(HIH) HTS(H) HML(M) SXS(M) SYS(M)' SZS(M)

$\begin{array}{lllllllll}1.220 E+06 & 6.00 E+01 & .00 E+00 & 2.20 E+02 & .00 E+00 & .00 E+00 & .00 E+00 & A\end{array}$

103. (H) IS DISTANCE TO .800E+01 (MG-MIN/M^3)

347. (M) IS DISTANCE TO .433E+00 (MG-MIN/M^3)

715. (H) IS DISTANCE TO .970E-01 (MG-MIN/H^3)

1414. (H) IS DISTANCE TO .483E-01 (MG-MIN/M^3) 
3299. (M) IS DISTANCE TO .207E-01 (MG-MIN/M^3)

7068. (H) IS DISTANCE TO .966E-02 (HG-MIN/M^3)

14137. (M) IS DISTANCE TO .483E-02 (MG-MIH/M^3)

32986. (M) IS DISTANCE TO .207E-02 (MG-MIN/M^3)

ALL OTHER INPUT

Stop - Program terminated. 


\section{APPENDIX F \\ CHEM_MACCS Developmental Verification Efforts}

\section{F.1 Verification}

MACCS was extensively checked and tested during the development of the code. Since the initial release of MACCS in 1990, it has been continuously maintained by Sandia National Laboratories under contract with the NRC. Maintenance activities have included documentation of code errors as reported by users, the correction of code errors, and the updating of code features as requested by users and funded by the NRC. It has been inspected line by line at the Idaho National Engineering Laboratory at the request of the NRC. ${ }^{1}$ The results of MACCS calculations have also been benchmarked against European, Japanese, and U.S. nuclear accident consequence codes and found to be in good agreement with other established consequence codes. ${ }^{2,3,4}$ MACCS has been used in many probabilistic risk assessments, including the NUREG-1150 study, ${ }^{5}$ and is widely used by Department of Energy facilities for safety analysis reports.

CHEM_MACCS was developed from MACCS 1.5.11.1. Some 58 subroutines associated with CHRONC and with radioactive decay calculations were deleted. Five common blocks were changed to include files simply to facilitate the code modifications. Minor changes were made to several routines simply to replace common blocks by include files. Section F.4 provides a list of all MACCS and CHEM MACCS subroutines and indicates the changes made to develop CHEM_MACCS from MACCS 1.5.

In the process of developing CHEM_MACCS from MACCS 1.5.11.1, several verification strategies were used to ensure compliance with the functional requirements in the software development plan. First, the development process was undertaken in stages with an example problem used at the end of each stage to verify that the coding tasks had been completed successfully. The test problem used for a particular stage varied according to the tasks undertaken, but the focus was on maintaining a working interim version of the code at all times. In this way, one could always go back to the working version from the previous stage if a particular coding effort became too difficult to debug.

To illustrate, one of the first stages undertaken was to strip out those subroutines that were required only for the CHRONC module of MACCS 1.5.11.1, since the CHRONC module was not to be a part of CHEM_MACCS. For this purpose, the test problems distributed with the MACCS User's Guide were run on MACCS 1.5.11.1 without CHRONC (EARLY input ENDAT2 set to .TRUE.). The runs were repeated after the CHRONC subroutines were deleted and the results of the before and after runs were compared to verify that the output results were identical.

In the next stages of development, the focus was-on getting CHEM_MACCS to calculate doses and health effects associated with chemical pathways. For these stages MACCS 1.5.11.1 was basically tricked into calculating chemical doses for a simple 
example problem by developing an appropriate DOSDATA.INP file and setting the skin and inhalation protection factors to appropriate values. Doses calculated by CHEM_MACCS at each stage of its development could then be compared with doses calculated by MACCS 1.5.11.1. A DOSDATA.INP file that was developed for this purpose is included in Section F.5.

At the same time, to verify correct implementation of probit equations into CHEM MACCS, the probit equations were approximated by the exponential hazard curve form required by MACCS 1.5.11.1 simply by matching the 5th and 50th percentiles. In conjunction with the user-defined DOSDATA.INP file, this permitted MACCS 1.5.11.1 to perform approximate chemical health effect calculations. Comparisons between MACCS 1.5.11.1 and CHEM_MACCS health effects predictions could then be made. The two codes did not (and were not expected to) give identical results; however, results usually agreed to 1-2 significant digits.

In developing the output option for land contamination, an analogous approach was taken. First, the existing EARLY output option 3, which gives the population exceeding a dose threshold, was exercised with a population density of one person per square kilometer. Then, in developing a new output option, a basis for comparison was maintained.

Finally, in January of 1995, when draft documentation and a test version of CHEM_MACCS had been distributed, a line-by-line check of all coding used to implement CHEM_MACCS models was undertaken. A few minor changes were made to the released version of CHEM_MACCS as a result of this line-by-line inspection.

\section{F.2 Validation}

Validation is concerned with whether a properly coded and functioning code fulfills its required specifications. Validation activities often include a comparison of code predictions with experimental results. No experiments are available against which to compare CHEM_MACCS predictions. This is fortunate because such experiments or accidents would have had to result in injuries or fatalities in order to have a basis for the most relevant comparisons.

Validation activities may also include running test problems provided with acquired codes and comparing the results with corresponding output files already provided. In this sense, the results of test calculations with CHEM_MACCS and comparisons with results obtained from another (SACRUNCH) model are relevant to the CHEM_MACCS validation.

Comparisons between CHEM_MACCS and SACRUNCH are documented in SAF-452-95-0022, which compares results of both codes for the vapor inhalation pathway given as $10,000-\mathrm{kg}$ VX-Vap release. ${ }^{6}$ In this case, acute lethality is the only consequence of concern. SAF-452-95-0022 demonstrates that air concentrations and early fatalities calculated by SACRUNCH and CHEM_MACCS are consistent with one another. 
To check the other pathways, the following approach was taken. The input deck in Sections F.6 and F.7 was developed to characterize a single-plume-segment release of $1,000 \mathrm{~kg}$ of VX-VAP and $1,000 \mathrm{~kg}$ of VX-LIQ. CHEM_MACCS was run with the ATMOS input IDEBUG $=1$ to obtain the centerline air and ground concentrations required in each of the pathway dose equations. Separate runs were required using VXVAP then VX-LIQ as the reference species. No evacuation or sheltering was modeled. Three EARLY input TYPE6 outputs were set to VIN1VX, VSK2VX, and LSK3VX to print the corresponding ground-level plume centerline doses.

The dose associated with the plume vapor inhalation pathway is found by Equation (3.3). As indicated in the input deck, $\mathrm{SFI}=0.41, \mathrm{BR}=\mathrm{BR}_{\mathrm{ACu}}=2.66 \mathrm{E}-04$ $\mathrm{m}^{3} / \mathrm{s}$, and $\mathrm{n}_{\mathrm{j}}=1$. RESCON was set to 0 to shut off the resuspension pathway. In the DOSDATA.INP file for dose VIN1VX and species VX-VAP, $W_{i j}=1$. For the modeled stationary individual, the exposure time TE is equal to the plume passage time TO. The doses were evaluated on the centerline, $J=1$. As indicated in Table F-1, the plumevapor inhalation doses calculated by CHEM_MACCS agree exactly with hand-calculated plume-vapor inhalation doses.

Table F-1 - CHEM_MACCS versus hand-calculated plume vapor inhalation doses

\begin{tabular}{|c|c|c|c|}
\hline $\begin{array}{c}\text { Downwind } \\
\text { Distance } \\
(\mathrm{km})\end{array}$ & $\begin{array}{c}\mathrm{ACt}_{\mathrm{i}} \\
\left(\mathrm{kg}-\mathrm{s} / \mathrm{m}^{3}\right)\end{array}$ & $\begin{array}{c}\text { CHEM_MACCS } \\
\text { VIN1VX }\end{array}$ & $\begin{array}{c}\text { Hand-Caiculated } \\
\text { VIN1VX }\end{array}$ \\
\hline 0.34 & $4.72 \mathrm{E}-02$ & $3.22 \mathrm{E}+02$ & $3.22 \mathrm{E}+02$ \\
1.41 & $8.77 \mathrm{E}-03$ & $5.99 \mathrm{E}+01$ & $5.99 \mathrm{E}+01$ \\
4.43 & $1.74 \mathrm{E}-03$ & $1.19 \mathrm{E}+01$ & $1.19 \mathrm{E}+01$ \\
13.7 & $3.17 \mathrm{E}-04$ & $2.17 \mathrm{E}+00$ & $2.17 \mathrm{E}+00$ \\
\hline
\end{tabular}

The CHEM_MACCS-calculated dose associated with the plume skin-vapor inhalation pathway is given by Equation (3.4). Noting from the EARLY input that on the protection factor SFVS $=0.41$ for vapor-skin contact, CHEM_MACCS-calculated plume-vapor skin doses agree exactly with hand-calculated plume-vapor inhalation doses as shown in Table F-2. 
Table F-2 - CHEM_MACCS versus hand-calculated plume vapor skin doses

\begin{tabular}{|c|c|c|c|}
\hline $\begin{array}{c}\text { Downwind } \\
\text { Distance } \\
(\mathrm{km})\end{array}$ & $\begin{array}{c}\mathrm{ACt}_{\mathrm{i}} \\
\left(\mathrm{kg}-\mathrm{s} / \mathrm{m}^{3}\right)\end{array}$ & CHEM_MACCS Dose & Calculated Dose \\
\hline 0.34 & $4.72 \mathrm{E}-02$ & $3.22 \mathrm{E}+02$ & $3.22 \mathrm{E}+02$ \\
1.41 & $8.77 \mathrm{E}-03$ & $5.99 \mathrm{E}+01$ & $5.99 \mathrm{E}+01$ \\
4.43 & $1.74 \mathrm{E}-03$ & $1.19 \mathrm{E}+01$ & $1.19 \mathrm{E}+01$ \\
13.7 & $3.17 \mathrm{E}-04$ & $2.17 \mathrm{E}+00$ & $2.17 \mathrm{E}+00$ \\
\hline
\end{tabular}

The CHEM MACCS-calculated dose associated with the liquid skin-vapor inhalation pathway is given by Equation (3.5). Noting from the EARLY input that on the protection factor SFLS $=0.41$ for liquid-skin contact while the skin deposition velocity is $\operatorname{SDV}_{\hat{i}}=0.01$, the CHEM_MACCS-calculated plume liquid-skin doses agree exactly with hand-calculated plume-vapor inhalation doses as shown in Table F-3.

Table F-3 - CHEM_MACCS versus hand-calculated plume liquid-skin doses

\begin{tabular}{|c|c|c|c|}
\hline $\begin{array}{c}\text { Downwind } \\
\text { Distance } \\
(\mathrm{km})\end{array}$ & $\begin{array}{c}\mathrm{ACt}_{\mathrm{i}} \\
\left(\mathrm{kg}-\mathrm{s} / \mathrm{m}^{3}\right)\end{array}$ & CHEM_MACCS Dose & Calculated Dose \\
\hline 0.34 & $4.64 \mathrm{E}-02$ & $3.42 \mathrm{E}+02$ & $3.42 \mathrm{E}+02$ \\
1.41 & $8.31 \mathrm{E}-03$ & $6.14 \mathrm{E}+01$ & $6.13 \mathrm{E}+01$ \\
4.43 & $1.56 \mathrm{E}-03$ & $1.15 \mathrm{E}+01$ & $1.15 \mathrm{E}+01$ \\
13.7 & $2.59 \mathrm{E}-04$ & $1.91 \mathrm{E}+00$ & $1.91 \mathrm{E}+00$ \\
\hline
\end{tabular}

To evaluate the effects of resuspension, the resuspension coefficient RESCON was arbitrarily set equal to 1.0 in the EARLY input. At this level the inhalation dose associated with resuspension far exceeds that associated with plume passage. The time at which the person enters into the spatial interval, $t_{1}$, is zero because the person is assumed to be in the interval at the time of plume arrival. The time at which the person leaves the spatial interval after plume departure, $t_{2}$, is 7 days because this is the upper limit for EARLY effects. The 7-day limit is used because there is no evacuation or sheltering modeled with these trials. To obtain the initial ground-level concentration, $G C_{i}$, the variable NUCOUT was set to VX-VAP for one run and was set to VX-LIQ for another run. Then the two values for the ground-level concentration were summed for a given distance. The reason for this is that the total ground-level concentration is the sum of the ground-level concentrations for both the vapor and liquid concentrations, because both contribute to the total concentration found at ground level at the initial time. 
The resuspended vapor inhalation dose calculated in CHEM_MACCS is given by Equation (3.7). Again, the doses were calculated on the centerline so $\mathrm{J}=1$. In the input deck, $\mathrm{SFI}=0.41, \mathrm{BR}=\mathrm{BR}_{\mathrm{ACU}}=2.66 \mathrm{E}-04 \mathrm{~m}^{3} / \mathrm{s}$, and $\mathrm{n}_{\mathrm{j}}=1$. The resuspension decay constant, $\lambda_{R}$, is calculated from the resuspension coefficient half-life, RESHAF. The calculated value used was $3.81 \mathrm{E}-06 \mathrm{~s}^{-1}$.

As indicated in Table F-4, CHEM_MACCS calculated resuspended plume-vapor inhalation doses are in agreement with hand-calculated resuspended plume-vapor inhalation doses.

Table F-4 CHEM_MACCS versus hand-calculated resuspended plume vapor inhalation doses

\begin{tabular}{|c|c|c|c|}
\hline $\begin{array}{c}\text { Downwind } \\
\text { Distance } \\
(\mathrm{km})\end{array}$ & $\begin{array}{c}\mathrm{GC}_{\mathrm{i}} \\
\left(\mathrm{kg} / \mathrm{m}^{2}\right)\end{array}$ & $\begin{array}{c}\text { CHEM_MACCS } \\
\text { VIN1VX }\end{array}$ & $\begin{array}{c}\text { Hand-Calculated } \\
\text { VIN1VX }\end{array}$ \\
\hline 0.34 & $5.11 \mathrm{E}-04$ & $8.25 \mathrm{E}+05$ & $8.25 \mathrm{E}+05$ \\
1.41 & $9.19 \mathrm{E}-05$ & $1.48 \mathrm{E}+05$ & $1.48 \mathrm{E}+05$ \\
4.43 & $1.73 \mathrm{E}-05$ & $2.79 \mathrm{E}+04$ & $2.80 \mathrm{E}+04$ \\
13.7 & $2.91 \mathrm{E}-06$ & $4.70 \mathrm{E}+03$ & $4.69 \mathrm{E}+03$ \\
\hline
\end{tabular}

The resuspended vapor-skin dose calculated in CHEM_MACCS is found by Equation (3.8). Following the same computational scheme described above for the resuspended vapor inhalation dose, Table F-5 shows that the CHEM_MACCS calculated resuspended plume-vapor skin doses agree with hand-calculated resuspended plume-vapor skin doses.

Table F-5 CHEM_MACCS versus hand-calculated resuspended plume vapor skin doses

\begin{tabular}{|c|c|c|c|}
\hline $\begin{array}{c}\text { Downwind } \\
\text { Distance } \\
(\mathrm{km})\end{array}$ & $\begin{array}{c}\mathrm{GC}_{\mathrm{i}} \\
\left(\mathrm{kg} / \mathrm{m}^{2}\right)\end{array}$ & $\begin{array}{c}\text { CHEM MACCS } \\
\text { VIN2VX }\end{array}$ & $\begin{array}{c}\text { Hand-Calculated } \\
\text { VIN2VX }\end{array}$ \\
\hline 0.34 & $5.11 \mathrm{E}-04$ & $8.25 \mathrm{E}+05$ & $8.25 \mathrm{E}+05$ \\
1.41 & $9.19 \mathrm{E}-05$ & $1.48 \mathrm{E}+05$ & $1.48 \mathrm{E}+05$ \\
4.43 & $1.73 \mathrm{E}-05$ & $2.79 \mathrm{E}+04$ & $2.80 \mathrm{E}+04$ \\
13.7 & $2.91 \mathrm{E}-06$ & $4.70 \mathrm{E}+03$ & $4.69 \mathrm{E}+03$ \\
\hline
\end{tabular}


To confirm the results produced by CHEM_MACCS for the continuous daily dose for the plume inhalation pathway, it was necessary to calculate the results by hand using Equation (3.9). The values for each of the variables are the same as those found for the plume vapor inhalation pathway calculations. However, an error in the code was detected. The answers generated by CHEM_MACCS were a factor of 60 below those calculated by hand. The error was traced to the variable UCFID in the routine EDOSIN.FOR and corrected. After the correction was made, the results generated by the code agreed with the hand calculations as shown in Table F-6.

Table F-6 - CHEM_MACCS versus hand-calculated continuous daily dose for plume vapor inhalation doses

\begin{tabular}{|c|c|c|c|}
\hline $\begin{array}{c}\text { Downwind } \\
\text { Distance } \\
(\mathrm{km})\end{array}$ & $\begin{array}{c}\mathrm{ACt} \\
\left(\mathrm{kg}-\mathrm{s} / \mathrm{m}^{3}\right)\end{array}$ & $\begin{array}{c}\text { CHEM_MACCS } \\
\text { VCCDVX }\end{array}$ & $\begin{array}{c}\text { Hand-Calculated } \\
\text { VCCDVX }\end{array}$ \\
\hline 0.34 & $4.72 \mathrm{E}-02$ & $2.87 \mathrm{E}-06$ & $2.87 \mathrm{E}-06$ \\
1.41 & $8.77 \mathrm{E}-03$ & $5.35 \mathrm{E}-07$ & $5.35 \mathrm{E}-07$ \\
4.43 & $1.74 \mathrm{E}-03$ & $1.06 \mathrm{E}-07$ & $1.06 \mathrm{E}-07$ \\
13.7 & $3.17 \mathrm{E}-04$ & $1.39 \mathrm{E}-08$ & $1.39 \mathrm{E}-08$ \\
\hline
\end{tabular}

The calculations for the continuous daily dose for resuspended vapor inhalation were also verified. Equation (3.10) is used to calculated this. Again, the variables are the same as those used for the previous calculations for a resuspended vapor where $\mathrm{GC}_{\mathrm{i}}$ is the sum of the ground-level concentrations for both of the liquid and vapor concentrations. After setting the TYPE6 output organ name to VCCDVX and its associated pathway to 'TOT LIF', the CHEM_MACCS-generated values matched those calculated by hand as indicated in Table F-7.

Table F-7 - CHEM_MACCS versus hand-calculated continuous daily dose for resuspended vapor inháatation doses

\begin{tabular}{|c|c|c|c|}
\hline $\begin{array}{c}\text { Downwind } \\
\text { Distance } \\
(\mathrm{km})\end{array}$ & $\begin{array}{c}\mathrm{ACt}_{\mathrm{i}} \\
\left(\mathrm{kg}-\mathrm{s} / \mathrm{m}^{3}\right)\end{array}$ & $\begin{array}{c}\text { CHEM_MACCS } \\
\text { VCCDVX }\end{array}$ & $\begin{array}{c}\text { Hand-Calculated } \\
\text { VCCDVX }\end{array}$ \\
\hline 0.34 & $4.72 \mathrm{E}-02$ & $2.87 \mathrm{E}-06$ & $2.87 \mathrm{E}-06$ \\
1.41 & $8.77 \mathrm{E}-03$ & $5.35 \mathrm{E}-07$ & $5.35 \mathrm{E}-07$ \\
4.43 & $1.74 \mathrm{E}-03$ & $1.06 \mathrm{E}-07$ & $1.06 \mathrm{E}-07$ \\
13.7 & $3.17 \mathrm{E}-04$ & $1.39 \mathrm{E}-08$ & $1.39 \mathrm{E}-08$ \\
\hline
\end{tabular}




\section{F.3 Conclusions}

Based on the extensive, well-documented checking and testing done during the development of MACCS 1.5.11.1, the fact that CHEM_MACCS is based on MACCS 1.5.11.1, with changes being carefully verified as discussed in Section F.2, and the favorable code-comparisons discussed in Section 6, it is concluded the CHEM_MACCS code adequately meets the requirements in its software development plan. 
F.4 Subroutine Changes from MACCS 1.5 to CHEM_MACCS

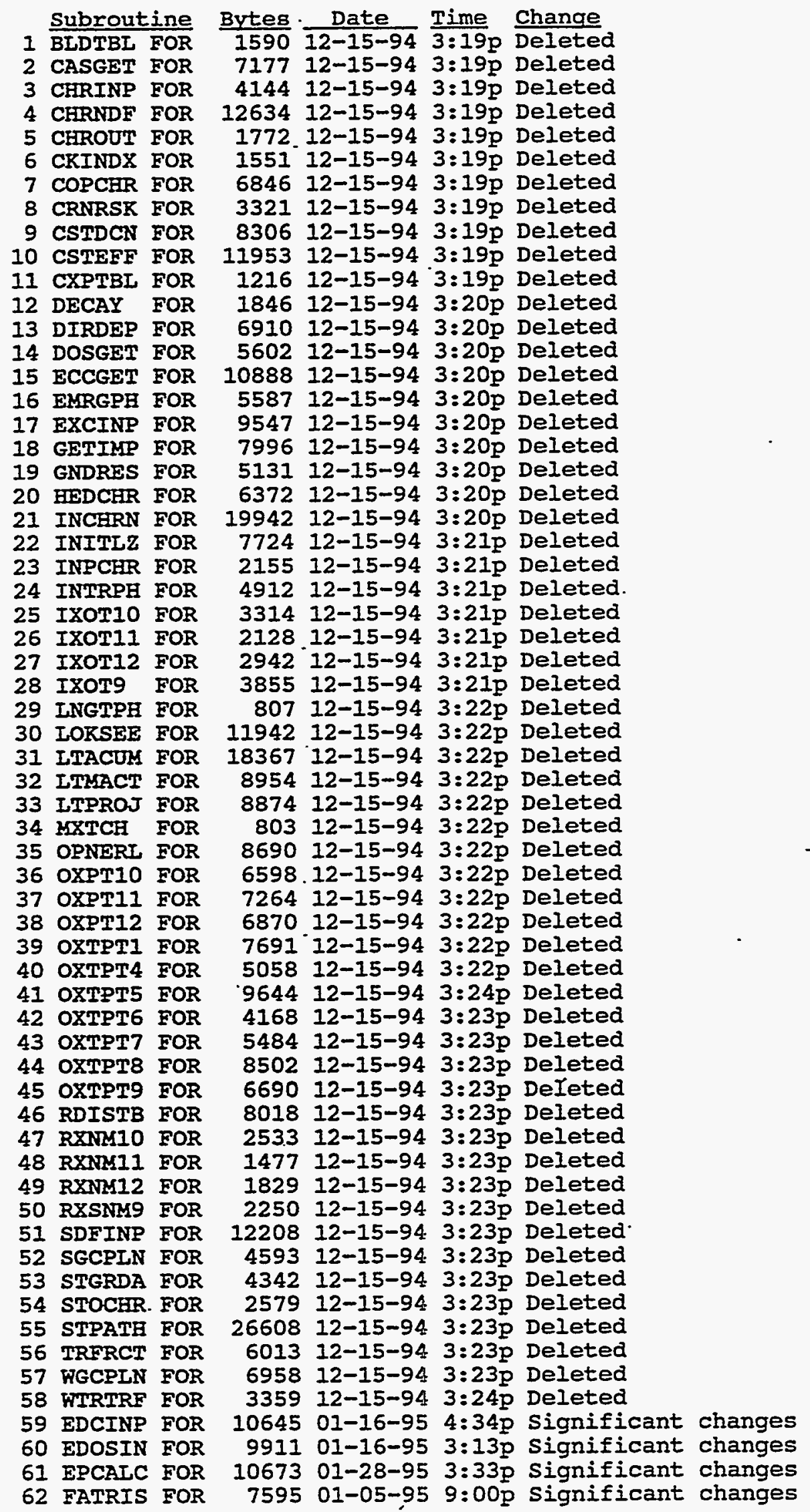


63 HEDEAR FOR 64 INJRIS FOR 65 INORGA FOR 66 INPISO FOR 67 RESNM3 FOR 68 CHEMACCFOR 69 ATMOUT FOR 70 CONTRT FOR 71 EARINP FOR

72 INOUT3 FOR

73 INOUT6 FOR

74 INPREI FOR

75 INPUT FOR

76 MACCS FOR

77 OUTCON FOR

78 STOEAR FOR

79 ERF FOR

80 GAMMIN FOR

81 GAMMP FOR

82 GCF FOR

83 GSER FOR

84 INOUTO FOR

85 INOUT9 FOR

86 OUTPTO FOR

87 OUTPT9 FOR

88 RESNMO FOR

89 RESNM9 FOR

90 INCDOS FOR

91 ABORT FOR

92 ADJTIM FOR

93 AREA FOR

94 ATMODL FOR

95 ATPROB FOR

96 BINSAM FOR

97 CANRIS FOR

98 CAUGRT FOR

99 CENACU FOR

100 CGETI FOR

101 CLSEIN FOR

102 CMPTBL FOR

103 COMPRS FOR

104 CONMET FOR

105 DAYHOU FOR

106 DISRAN FOR

107 DISTI FOR

108 DOICDF FOR

109 DOCCDF FOR

110 EAROUT FOR

111 EFFGET FOR

112 EGEOM FOR

113 EMOVE FOR

114 ERRFIL FOR

115 ERRLOC FOR

116 ESTAT FOR

117 EVNETW FOR

118 EVRADI FOR

119 EVROOT FOR

120 EXPINT FOR

121 FSGY FOR

122 FSGZ FOR

123 GNBINI FOR

124 GNBIN2 FOR

125 IGETI FOR

126 IGETN FOR

127 ILOG10 FOR
9904 01-16-95 12:28psignificant changes

8086 01-01-95 11:32asignificant changes

4408 01-04-95 2:25p Significant changes

7207 01-07-95 8:33a significant changes

2319 01-06-95 4:36p Significant changes

24286 01-17-95 9:25a Minor changes from MACCS.FOR

30218 12-21-94 12:14pMinor changes

7030 12-20-94 7:09p Minor changes

5516 01-13-95 3:23p Minor changes

5458 01-16-95 4:32p Minor changes

6872 01-16-95 4:32p Minor changes

11121 12-21-94 12:17pMinor changes

6108 12-20-94 7:08p Minor changes

24239 12-18-94 4:52p Minor changes to CHEMACCS.FOR

1043 12-21-94 10:14aMinor changes

2789 01-09-95 3:33p Minor changes

560 10-26-94 2:51p New

1423 10-26-94 2:51p New

1036 10-26-94 2:52p New

1841 10-26-94 2:52p New

1452 10-26-94 2:52p New

4827 01-16-95 2:37p New

4755 01-16-95 5:22p New

5842 01-16-95 2:33p New

2806 01-13-95 5:36p New

1476 01-16-95 2:35p New

1496 01-16-95 2:36p New

6159 01-02-95 4:49p Unchanged

1553 12-15-94 3:19p Unchanged

1866 12-15-94 3:19p Unchanged

629 12-15-94 3:19p Unchanged

1823 12-15-94 3:19p Unchanged

2088 12-15-94 3:19p Unchanged

5166 12-15-94 3:19p Unchanged

8211 01-01-95 11:35aUnchanged

1676 12-15-94 3:19p Unchanged

2919 01-02-95 4:38p Unchanged

5575 12-15-94 3:19p Unchanged

2625 12-15-94 3:19p Unchanged

913 12-15-94 3:19p Unchanged

962 12-15-94 3:19p Unchanged

1795 12-15-94 3:19p Unchanged

2259 12-15-94 3:20p Unchanged

756 12-15-94 3:20p Unchanged

1497 12-15-94 3:20p Unchanged

4786 12-15-94 3:20p Unchanged

2699 12-15-94 3:20p Unchanged

9322 01-01-95 12:32pUnchanged

3997 12-15-94 3:20p Unchanged

7730 01-01-95 12:05pUnchanged

9890 01-02-95 4:40p Unchanged

527 12-15-94 3:20p Unchanged

519 12-15-94 3:20p Unchanged

10047 01-01-95 12:05pUnchanged

7307 12-15-94 3:20p Unchanged

3102 12-15-94 3:20p Unchanged

2946 12-15-94 3:20p Unchanged

648 12-15-94 3:20p Unchanged

1444 12-15-94 3:20p Unchanged

1303 12-15-94 3:20p Unchanged

1680 12-15-94 3:20p Unchanged

3395 12-15-94 3:20p Unchanged

6005 12-15-94 3:20p Unchanged

2781 12-15-94 3:20p Unchanged

251 12-15-94 3:20p Unchanged 
128 IMDIGT FOR 129 IMIGCL FOR 130 IMNTGR FOR 131 IMREAI FOR 132 INACAN FOR 133 INCREM FOR 134 INDFAC FOR 135 INEFAT FOR 136 INEINJ FOR 137 INEVAC FOR 138 INMISC FOR 139 INOUT1 FOR 140 INOUT2 FOR 141 INOUT4 FOR 142 INOUT5 FOR 143 INOUT7 FOR 144 INOUT8 FOR 145 INPBEG FOR 146 INPDIS FOR 147 INPDRY FOR 148 INPEMR FOR 149 INPEND FOR 150 INPEXP FOR 151 INPGEO FOR 152 INPIRS FOR 153 INPMI FOR 154 INPM2 FOR 155 INPM 3 FOR 156 INPM4 FOR 157 INPM5 FOR 158 INPMET FOR 159 INPOPT FOR 160 INPOPU FOR 161 INPWAR FOR 162 INPWET FOR 163 IGETI FOR 164 IGETN FOR 165 NATCH FOR 166 MXXCLR FOR 167 MXXCPU FOR 168 MXXDAT FOR 169 MXXOPN FOR 170 NOTFOU FOR I71 OPNFII FOR 172 OUTPTI FOR 173 OUTPT2 FOR 174 OUTPT3 FOR 175 OUTPT4 FOR 176 OUTPT5 FOR 177 OUTPTE FOR 178 OUTPT7 FOR 179 OUTPTS FOR 180 OUTPUT FOR 181 PIMIRIS FOR 182 POL2 EOR 183 PRINT FOR 184 PUTSTG FOR 185 PUTSTM FOR 186 QUANTI FOR 187 RANDOM FOR 188 RANSAM FOR 189 RDSTRG FOR 190 READI FOR 191 READ2 FOR 192 REDSTG FOR
799 12-15-94 3:20p Unchanged 420 12-15-94 3:20p Unchanged 648 12-15-94 3:20p Unchanged 3323 12-15-94 3:20p Unchanged 6311 12-15-94 3:20p Unchanged 5335 01-02-95 4:51p Unchanged 3285 01-01-95 11:51aUnchanged 3999 12-18-94 4:35p Unchanged 4774 12-18-94 4:39p Unchanged 7547 12-15-94 3:21p Unchanged 5938 12-15-94 3:21p Unchanged 7856 12-15-94 3:21p Unchanged 2394 12-15-94 3:21p Unchanged 6943 12-15-94 3:21p Unchanged 4556 12-15-94 3:21p Unchanged 8070 12-15-94 3:21p Unchanged 7861 12-15-94 3:21p Unchanged 11022 12-15-94 3:21p Dnchanged 2792 12-15-94 3:21p Unchanged 1771 12-15-94 3:21p Unchanged 6681 12-15-94 3:21p Unchanged 2529 12-15-94 3:21p Unchanged 2140 12-15-94 3:21p Unchanged 2763 12-15-94 3:21p Unchanged 2125 12-15-94 3:21p Unchanged 2398 12-15-94 3:21p Unchanged 2615 12-15-94 3:21p Unchanged 1357 12-15-94 3:21p Unchanged 8924 12-15-94 3:21p Unchanged 2907 12-15-94 3:21p Unchanged 2761 12-15-94 3:21p Unchanged 3204 12-15-94 3:21p Unchanged 7926 12-15-94 3:21p Unchanged 1829 12-15-94 3:21p Unchanged 1547 12-15-94 3:21p Unchanged 5353 12-15-94 3:22p Unchanged 2428 12-15-94 3:22p Unchanged

729 12-15-94 3:22p Unchanged 2677 12-15-94. 3:22p Unchanged 1937 12-15-94. 3:22p Unchanged 3223 12-15-94 3:22p Unchanged 2477 12-15-94. 3:22p Unchanged 487 12-15-94: 3:22p Unchanged 912 12-15-94. 3:22p Unchanged 7113 12-15-94. 3:22p Unchanged 4106 12-15-94: 3:22p Unchanged 8988 12-15-94. 3:22p Unchanged 6008 12-15-94. 3:22p Unchanged 6649 12-15-94: 3:22p Unchanged 5515 01-01-95 11:29aUnchanged 6309 12-15-94 3:22p Unchanged 7025 12-15-94 3:22p Jnchanged 1860 12-15-94 3:22p Unchanged 4502 12-15-94 3:23p Unchanged 1691 12-15-94 3:23p Unchanged 15522 12-15-94 3:23p Unchanged 4345 12-15-94 3:23p Unchanged 3626 12-15-94 3:23p Unchanged 3072 12-15-94 3:23p Unchanged 2489 12-15-94 3:23p Unchanged 4003 12-15-94 3:23p Unchanged 6199 12-15-94 3:23p Unchanged 5786 12-15-94 3:23p Unchanged 6576 12-15-94 3:23p Unchanged 564 12-15-94 3:23p Unchanged 
193 RELZON FOR

194 RESNMI FOR

195 RESNM2 FOR

196 RESNM4 FOR

197 RESNM5 FOR

198 RESNM6 FOR

199 RESNM7 FOR

200 RESNMB FOR

201 RGETI FOR

202 RGETN FOR

203 SEARCE FOR

204 SIGTEX FOR

205 SOIID FOR

206 SORT FOR

207 USRSUP FOR

208 VELADJ FOR

209 WASHOU FOR

210 WBNDRY FOR

211 WBNMET FOR

212 WGTMET FOR

213 WINCTM FOR

214 WNDRZB FOR

215 HRANBN FOR

216 WRDMET FOR

217 WRIT80 FOR

218 WSAMPI FOR

219 ZERREM FOR
7714 01-01-95 12:04pUnchanged 1717 12-15-94 3:23p Unchanged 1147 12-15-94 3:23p Unchanged 1528 12-15-94 3:23p Unchanged 1649 12-15-94 3:23p Unchanged 1630 01-03-95 4:30p Unchanged 1342 12-15-94 3:23p Unchanged 1601 12-15-94 3:23p Unchanged 5997 12-15-94 3:23p Unchanged 2787 12-15-94 3:23p Unchanged 2033 12-15-94 3:23p Unchanged 435 12-15-94 3:23p Unchanged 579 12-15-94 3:23p Unchanged 1469 12-15-94 3:23p Unchanged 1912 12-15-94 3:23p Unchanged 997 12-15-94 3:23p Unchanged 537 12-15-94 3:23p Unchanged 2090 12-15-94 3:23p Unchanged 13285 12-15-94 3:23p Unchanged 3235 12-15-94 3:23p Unchanged 497 12-15-94 3:23p Unchanged 2861 12-15-94 3:23p Unchanged 1544 12-15-94 3:24p Unchanged 4726 12-15-94 3:24p Unchanged 2330 12-15-94 3:24p Unchanged 1551 12-15-94 3:24p Unchanged 1269 12-15-94 3:24p Unchanged 
F.5 DOSDATA.INP File Used for Developmental Verification

HACCS File DOSDATA.IHP: Changed by E. HASKIH18-JAK-94, 13:00:00

VX dose conversion factors for CHEM MACCS Version 1.0.2 3 DOSES (ORGAKS) DEFIHED IN THIS FILE:

DE SKH VXL

IH SKN VXV

IH CDD $V X V$

$v x-210$

2 CHEMICALS (HUCLIDES) DEFINED IN THIS FILE:

VX-VAP

CLOUDSHINE GROUHD GROUHD GROUHD INHALED 'IHHALED SHIME 8HR SHINE TDAY SHINE RATE ACUTE CHRONIC

$\mathrm{VX}$-LIO

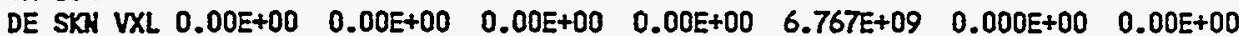

IH SKA VXV $0.00 E+00 \quad 0.00 E+00 \quad 0.00 E+00 \quad 0.00 E+00 \quad 0.000 E-00 \quad 0.000 E+00 \quad 0.00 E+00$

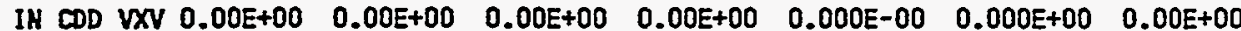

VX-VAP

DE SKN VXL $0.00 E+00 \quad 0.00 E+00 \quad 0.00 E+00 \quad 0.00 E+00 \quad 0.000 E-00 \quad 0.000 E+00 \quad 0.00 E+00$

$\begin{array}{llllllll}\text { IM SKN VXV } & 0.00 E+00 & 0.00 E+00 & 0.00 E+00 & 0.00 E+00 & 6.250 E+07 & 0.000 E+00 & 0.00 E+00\end{array}$

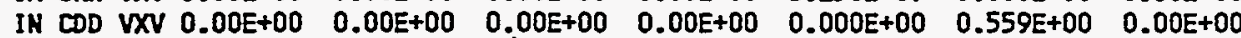




\section{F.6 ATMOS Input File}

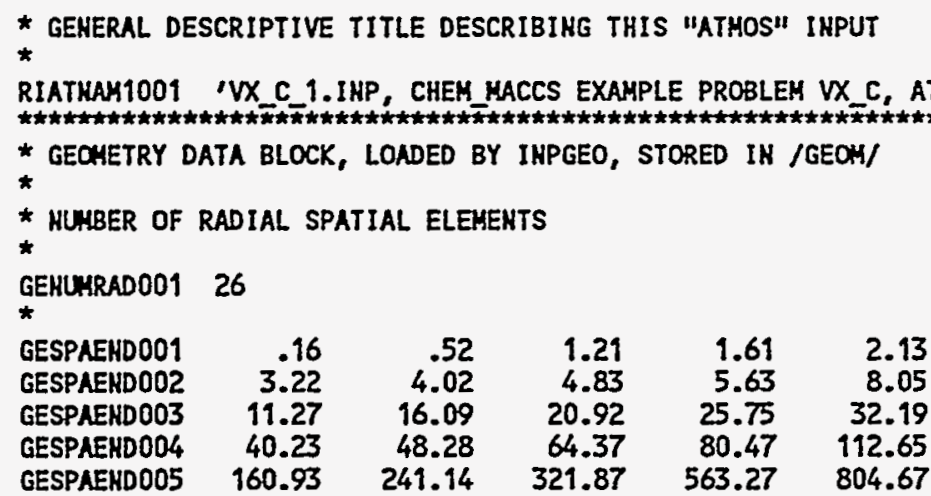

$\begin{array}{llll}241.14 & 321.87 & 563.27 & 804.67\end{array}$

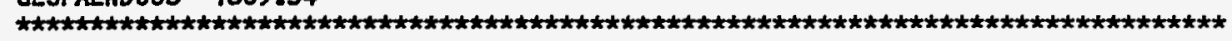

* CHeRICAL SPECIES DATA BLOCK, LOADED BY IMPISO, STORED IH /ISOHAH/

* NuMBer of chemical species

*

ISHUHISOOOI 2

*

* CHEMICAL SPECIES DATA

$\star$

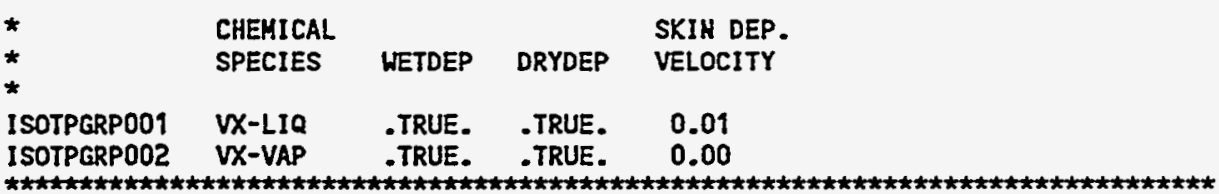

* YET DEPOSITIOH DATA BLOCK, LOADED BY IHPWET, STORED IN /WETCON/

$\star$

* HASHOUT COEFFICIENT hLABER OHE, LIMEAR FACTOR

WDCUASHTO01 9.5E-5 (JON HELTON AFTER JONES, 1986)

*

* UASHOUT COEFFICIENT NUMBER TWD, EXPOHENTIAL FACTOR

HDCUASH2001 0.8 (JON HELTON AFTER JOHES, 1986)

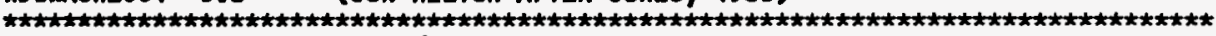

* DRY DEPOSITION DATA BLOCK, LOADED BY INPDRY, STORED IN /DRYCON/ .

* humber of particle size groups

DDNPSCRP001 2

* DEPOSITIOH VELOCITY OF EACH PARTICLE SIZE GROUP (H/S)

DDVEPOSOOT $0.001 \quad 0.01$

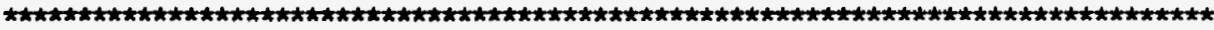

* DISPERSION PARAMETER DATA BLOCK, LOADED BY IHPDIS, STORED IN /DISPY/, /DISPZ/

$\star$ SIGH $=A x \star \star$ B WHERE A AND B VALUES ARE FROM TADHOR AND GUR (1969)

*

* LIMEAR TERH OF THE EXPRESSIOH FOR SIGMA-Y, 6 STABILITY CLASSES

*

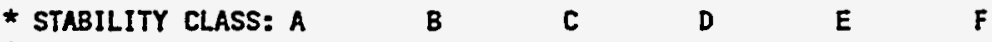

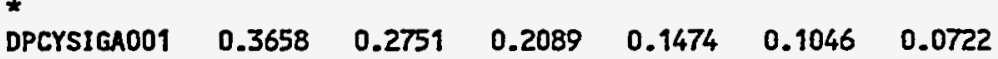

* EXPONENTIAL TERH OF THE EXPRESSION FOR SIGHA-Y, 6 STABILITY CLASSES

* EXPONENTIAL TERH OF THE EXPRESSION FOR SIQXA-Y, 6 STABILITY CLASSES

* STABILITY Class: A B B $\quad$ D E

$\begin{array}{lllllll}\text { DPCYSIGBO01 } & .9031 & .9031 & .9031 & .9031 & .9031 & .9031\end{array}$

* LIHEAR TERH OF THE EXPRESSION FOR SIGHA-Z, 6 STABILITY CLASSES

$+$

* STABILITY Class: A B C , D E E 


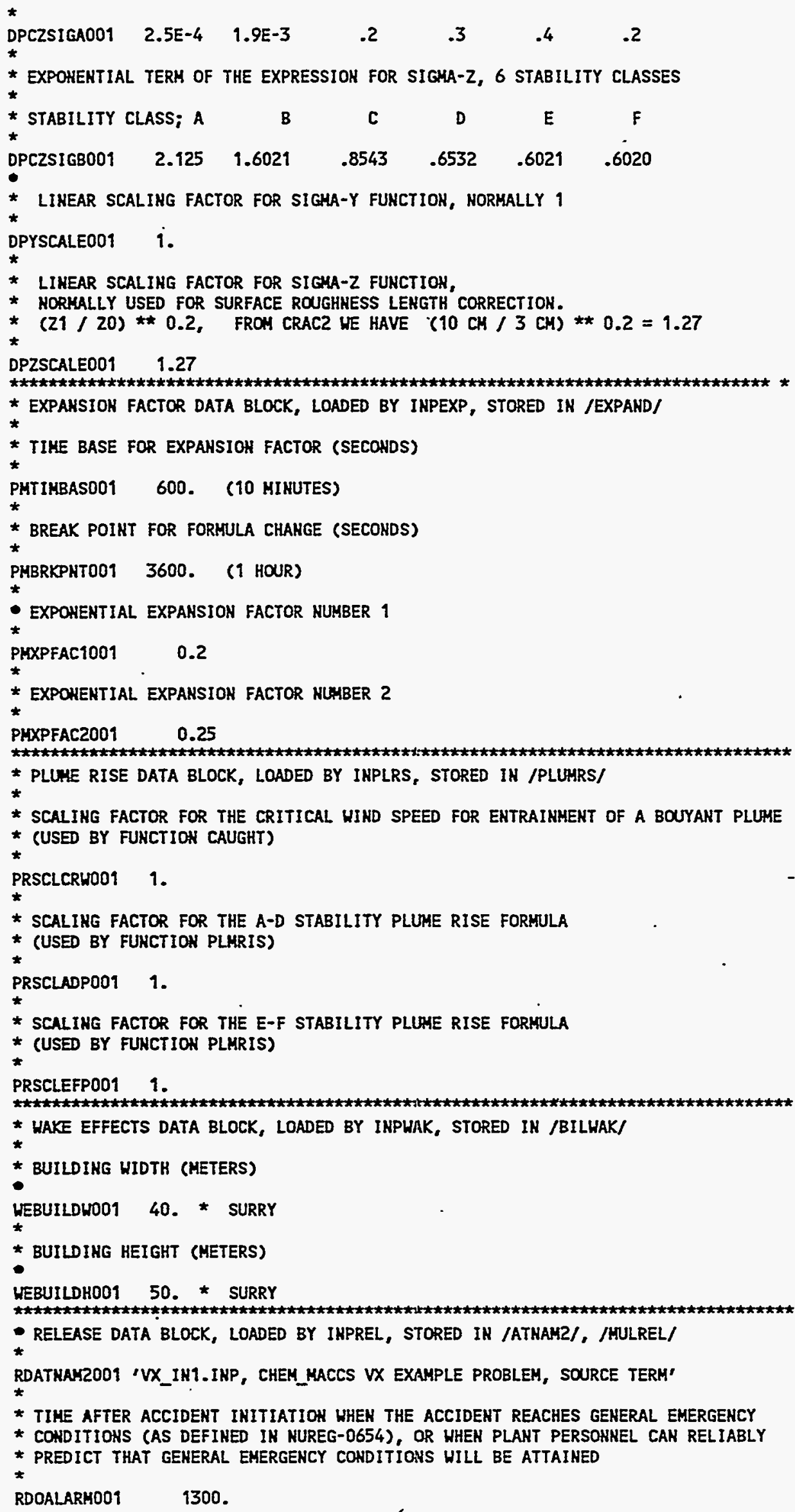


* number of pluhe segments that are released RDNUMRELO01

* SElection of RISK dominakt PLLhe

RDMAXRISOO1 1

* REFEREHCE TIHE fOR DISPERSION

*

RDREFTIHOO1 $\quad 0.50$

* heAT CONTENT OF THE RELEASE SEGHentS (H)

* a Value specified for each of the release seghents

RDPLHEATOO1 1.E+6

* height of the PLUMe SEgments at RELEASE (h)

* a value specified for each of the release segments

RDPLHITE001

0.

* DURATION OF THE PLUME SEGMENTS (S)

* a VAlue SPECIfIEd for EaCH of the RELEASE SEguentS

*

RDPLUDUR001 1800

*

* time of release for each pluae

* a value specified for each of the release seghents

RDPDELAYOOT 0 .

* Particle size distribution of each chemical species

* THE fRACTIOHS FOR EACH CHEHICAL SPECIES (ROW) hUST SUH TO OHE.

*

*

*

RDPSDISTOOI

RDPSDISTOD2

$0.001 \mathrm{~m} / \mathrm{s} \quad 0.01 \mathrm{~m} / \mathrm{s}$

0.0

1.0

$* V X-L I Q$

1.0

0.0

$\star V X-V A P$

* inventory of each chemical species subject to release

* CHEMICAL QUANTITY

* SPECIES RELEASED

* (kg)

RDCORIHVOOI VX-LIQ $1.000 E+03$

RDCORIHVOO2 VX-VAP. $\quad 1.000 E+03$

* scalihg factor to adjust the inventory

RDCORSCAOO1 1.000

* RELEASE fRActIONS FOR CHEMICAL SPECIES bY PLUHE

$\star$

* Pllater VX-liq vX-VAP

RDRELFRCOO1 1.0E+0 1.0E+0

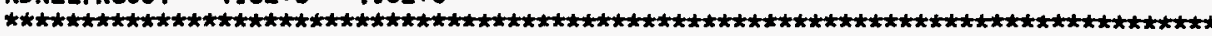

* OUTPUT CONTROL DATA BLOCK, LOADED BY INPOPT, STORED IN /STOPME/, /ATMOPT/

$\star$

* flag to indicate that this is the last program in the series to be RUN

OCENDAT1001 . FALSE. (SET THIS VALUE TO .TRUE. TO SKIP EARLY AND CHRONC)

*

OCIDEBUGOOI 1

* haMe of the chemical species to be listed on the dispersion listings

OCNUCOUTOD1 VX-VAP

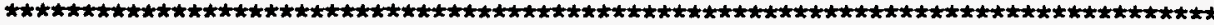

* heteorological SAMPLIMg DATA BLOCK 
*

* meteorological sahpling option coDe:

-

* HETCOO $=1$, USER SPECIFIED DAY AHD HOUR IH THE YEAR (FROH MET FILE),

- 2, WeATHER chtegory bIN SAMPLING,

* 3, 120 hOURS OF HEATHER SPECIFIED ON THE ATMOS USER INPUT FILE,

* 4, COHSTAHT HET (BOUNDARY WEATHER USED FROA THE START),

* 5, STRATIFIED RANDON SAMPLES FOR EACH DAY OF THE YEAR.

HIHETCOO001 4 (BOUNDARY WEATHER USED FROH THE START)

-

- hour of day InTERVAl IN hHICH accident mUSt begin

* HRIN1A HRINTB

*

HIHRINITOOI $\quad 1.0 \quad 24.0$

* Last spatial IHTERVAl for heasured weatier

-

HZLIHSPAOOI 25

$\star$

* BOUHDARY WEATHER HIXING LAYER HEIGHT

*

M2BHDHXHOO1 1000 . (HETERS) -

*

* BOUMDARY uEATHER STABILITY CLASS IHDEX

$\star$

HZIBDSTBOOI 4 (D-STABILITY)

$\rightarrow$

* BOUNDARY vEATHER RAIH RATE

MZBNDRANOOI O. (MH/HR)

*

* BOUHDARY WEATHER UIHD SPEED

HZBKDHND0015. (A/S)

- start day IH the year for the single heather sequence

MBISTRDYOOI 157 (START TIME FOR PEAK ECONOMIC COST OF SAHPLE PROBLEM A)

- start hour In the DAy for the single ueather sequence

MBISTRHROO1 10 (START TIHE FOR PEAK ECONOMIC COST OF SAMPLE PROBLEM A) 


\section{F.7 EARLY Input File}

* genERAL DESCRIPTIVE TITLE DESCRIBING thIS "EARLY" IHPUT fILE

MIEAKRM1001 'VX_A_2.IHP, CHEH_HACCS EXAMPLE PROBLEH VX_A, EARLY IHPUT'

$\bullet$

* DISPERSIOH hOOEL OPTIOH COOE: 1 * STRAight LIHE

$\star$

MIIPLLMEOO1 1 (STRAIGHT LINE PLLAE)

$\star$

* number of fihe grid subdivisions used by the hoOel

*

MINLHFIHOO1 7 (3,5 OR 7 ALLONED)

* LEVEl of deBUG OUTPUT REQUIREd, hORHAL RUNS SHOULD SPECIFY ZERo

*

MIIPRIIHTOOI 0 (TURN OFF THE DEBUG PRIKT)

* logical flag signifyimg that the breakdonn of risK by weather category

* BIN ARE TO BE PRESENTED TO SHON THEIR RELATIVE CONTRIBUTION TO THE MEAK

$\star$

*

*

MIRISCATOOI .FALSE.

$\star$

* flag imdicatikg if uind-roses froh athos are to be overriddeh

*

MIOVRRIDOOI .FALSE. (USE THE HIND ROSE CALCULATED FOR EACH WEATHER BIN)

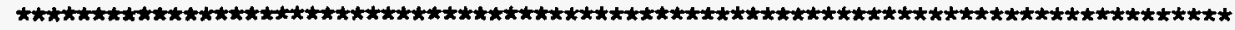
* POPULATION DISTRIBUTIOH DATA BLOCK, LOADED BY INPOPU, STORED IN /POPDAT/

*

PDPOPFLGOO UNIFORH

PDIBEGIMO01 1 (SPATIAL INTERVAL AT HHICH POPULATIOH BEGINS)

PDPOPDEMOO1 50. (POPULATIOH DEMSITY (PEOPLE PER SQUARE KILOMETER))

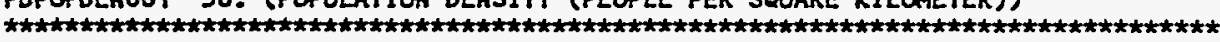
* DOSE DEFIHITION DATA BLOCK, LOADED BY INORGA, STORED IH /EARDIH/ AND /ORGHAM/

*

* huHber of doses defined for health efFects

$\star$

CONUHORGOO1 4

$\star$

* DOSE PATHHAY CONCEHTRATIOH

* HAME fOR DOSE EXPOHENT

COORGYAMDO1 'VINIVX' 'INH ACU' 1.0

COORGHAMOO2 'VSK2VX'. 'CLD' 1.0

COORGHAMOO3 'LSK3VX' 'SKH ACU' 1.0

COORGHAOO4 'VCDDVX' 'INH LIF' 1.0

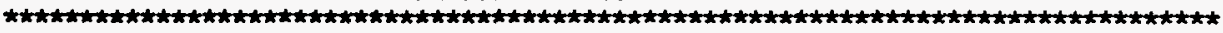

* SHIELDIHg AKD EXPOSURE FACTORS, LOADED BY IHDFAC, STORED IK /EADFAC/

* three values of each protection factor are supplied,

* OHE FOR EACH TYPE OF ACTIVTY:

* ACTIVITY TYPE:

* 1 - EVACUEES HHILE HOVIHG

* 2 - hORMAL aCTIVITY IH SHELTERING aHD EVACUATIOK zONE

* 3 - SHELTERED ACTIVITY

* PROTECTION FACTOR FOR INHALATION

SEPROTIHOO1 $\quad 1 . \quad 0.41 \quad 0.33$ * VALUES FOR NORMAL ACTIVITY AND

$\star \quad$ SHELTERIHG SELECTED BY HRC STAFF

$\star$

- BREATHIHG RATE AND REFEREHCE BREATHIHG RATE (CUBIC HETERS PER SECOND)

SEBRRATE001 2.66E-4 2.66E-4 2.66E-4 * BREATHIHG RATE

SEBRRATEOO2 2.66E-4 2.66E-4 2.66E-4 * REFERENCE BREATHING RATE

* SKIN PROTECTIOH FACTOR 
* VALUES FOR NORMAL ACTIVITY AND SHELTERIHG SELECTED BY NRC STAFF 
* SHELTER DURATIOH IH THE OUTER SHELTER ZONE (SECONDS FROH TAKING SHELTER)

SRSHELT2001 86400. (Entire plume duration plus)

* DURATIOH OF THE EHERGEHCY PHASE (SECONDS FROM PLUME ARRIVAL)

*

SREKDEMPOD1 604800. (ONE VEEK)

$\star$

* critical dose for relochtion decisions

*

SRCRIORG001 'VIKIVX'

*

* hOT SPOT RELOCATION TIHE (SECONDS FROH PLUHE ARRIVAL)

*

SRTIHHOTO01 43200. (OHE-HALF DAY)

*

* horhal RELOcatioh time (SECONDS froh PLUMe arRIVAL)

*

SRTIHHRHO01 86400. (OHE DAY)

$*$

* HOT SPOT RELOCATION DOSE CRITERION THRESHOLD (SIEVERTS)

SRDOSHOTO01 0.5 (50 REH DOSE TO YHOLE BOOY IN 1 WEEK TRIGGERS RELOCATION)

*

* normal relocation dose CRITERIOH THRESHOLd

*

SRDOSHRHOO1 0.045 (25 MG/M^3-S VAP AIR COHC DOSE IN 1 WK TRIGGERS RELOCATION)

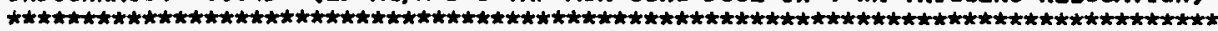

* EARLY FATALITY MOOEL PARAMETERS, LOADED BY INEFAT, STORED IH /EFATAL/

* MUMBER of EARLY FATALITY EFFECTS

EFHLIEFAOO1 3

$\begin{array}{llrcc}* & \text { ORGHAY } & \text { EFFACA } & \text { EFFACB } & \text { EFFTHR } \\ * & & & & \\ \text { EFATAGRP001 } & \text { 'VIHIVX' } & 15.0 & 6.3 & 8.22 \\ \text { EFATAGRP002 } & \text { 'VSK2VX' } & 200.0 & 5.5 & 100 .\end{array}$

EFATAGRPO03 'LSK3VX' $\quad 5.0 \quad 5.5 \quad 2.5$

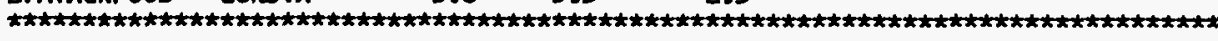

* EARLY IHJURY hODEL PARAHETERS, LOADED BY INEINJ, STORED IH /EIHJUR/

$\star$

* MUHBER OF EARLY INJURY EFFECTS

$\star$

EIHLAEIHOO1 3

* EIHAHE ORGKAH EISUSC EITHRE EIFACA EIFACB

EIHJUGRPOO1 'CLP, PRL, COKVL' 'LSKBVX'

EIHJUGRPOO2 'HIOSIS/RHIKORRH' 'VIHIVX'

EIHJUGRPOO3 'SWEATIHG/FASCIC' 'VSK2VX' $1.05 .0 \quad 10.0 \quad 5.5$

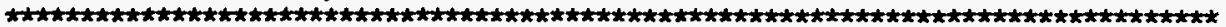

* aCUte exposure cahcer paraheters, loaded by INaCAN STORED IN /ACANCR/.

* humber of acute exposure cahcer effects

LCHUHACAOOI 1

t

* THRESHOLD DOSE FOR APPLYING DDREFA

LCODTHREOO1 0.

* DOSE THRESHOLd FOR LINEAR DOSE RESPOHSE

LCACTHREOOT

* ACHAME ORGMAH ACSUSC DOSEFA DOSEFB CFRISK CIRISK DDREFA

LCAMCERSO01 'CAHCER' 'VCODVX' $1.0 \quad 1.0 \quad 0.0 \quad 0.00$

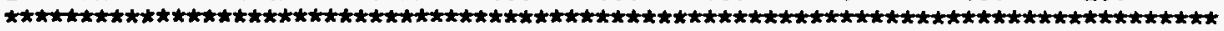

* RESULT 1 OPTIONS BLOCK, LOADED BY INOUT1, STORED IN /INOUT1/

* TOTAL NUMBER OF A GIVEN EFFECT (LATENT CAHCER, EARLY DEATH, EARLY INJURY)

$\star$ 
* hUHBER OF DESIRED RESULTS OF THIS TYPE TYPEIMUMBER 5

TYPEIOUTOO1

TYPE1OUTO02

TYPE1OUTO03

TYPE10UT004

TYPE1OUTOO9

'ERL FAT/TOTAL'

126 CCDF (O TO 1000 MILES)

'ERL INJ/CLP, PRL, CONVL' 126

'ERL INJ/MIOSIS/RHIHORRH' 926

'ERL INJ/SWEATING/FASCIC' 126

'CAN INJ/CANCER'

126

* RESULT 2 OPTIONS BLOCK, LOADED BY IMOUT2, STOPED IH IIHOUT2/

* FURTHEST DISTANCE AT WHICH A GIVEN RISK ÓF EARLY DEATH IS EXCEEDED.

* muiber of desired results of this type

TYPEZNUHBER 1

$\star$

*

$\star$

TYPEZOUTO01 0. CCDF *DISTANCE AT WHICH ANY FATALITIES OCCURRED

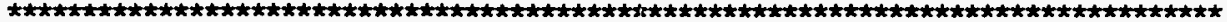

- RESULT 3 OPTIOHS BLOCK, LOADED BY IHOUT3, STORED IH /IHOUT3/

* HUABER OF PEOPLE WHOSE DOSE EXCEEDS A GIVEN THRESHOLD.

-

* NUHBER OF DESIRED RESULTS OF THIS TYPE

$\star$

TYPEBNUABER 4

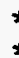

*

TYPE3OUTOOT 'VIHIVX' 8.22

TYPE3OUTO02 'VSK2VX' 100.

TYPESOUTO03 'LSK3VX' 2.5

TYPE3OUT004 'VCDDVX' 0.0

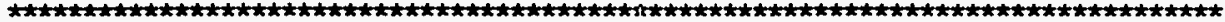

* RESULT 4 OPTIOHS BLOCK, LOADED BY IHOUTf, STORED IN /INOUT4/

* 360 DEGREE AVERAGE RISK OF A GIVEN EFFECT AT A GIVEN DISTANCE.

$\star$

* POSSIBLE TYPES OF EFFECTS aRE:

*

- 'erl fat/total'

- 'ERL IHJ/IHJURY NAHE'

* 'CAH FAT/CAHCER HAHE'

* 'cah fat/total'

- huaber of desired. REsults. of this_tyPE

*

TYPE4NUABER 4

$+$

*

$\begin{array}{lcl}\text { TYPE4OUTO01 } & 1 & \text { 'ERL FAT/TOTAL' } \\ \text { TYPE4OUT002 } & 2 & \text { 'ERL IHJ/CLP, PRL, COHVL' } \\ \text { TYPE4OUTO03 } & 3 & \text { 'ERL IHJ/MIOSIS/RHIHORRH' } \\ \text { TYPE4OUT004 } & 4 & \text { 'ERL IHJ/SHEATIHG/FASCIC' } \\ \text { *TYPE4OUT005 } & 5 & \text { 'CAN IHJ/CANCER' }\end{array}$

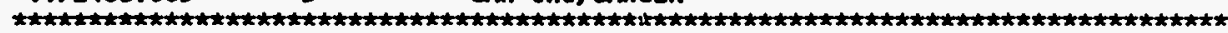

- RESULT 5 OPTIONS BLOCK, LOADED BY INOUT5, STORED IH /INOUT5/

$\star$

* total population dose betheEn tho distances.

$\star$

* husBer of DESIRED RESULTS OF THIS TYPE

*

TYPESHUMBER 1

?

* DOSE IIDIS5 I20IS5

TYPE5OUTOO1 'VCDDVX' 1 12 (0-10 HILES)

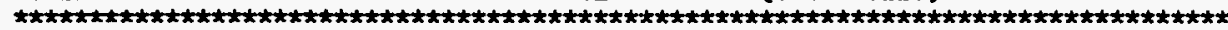

* RESULT 6 OPTIONS BLOCK, LOADED BY IHOUT6, STORED IN /INOUT6/

$\star$

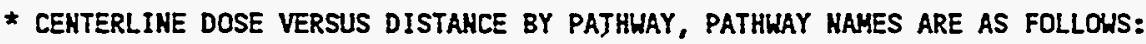




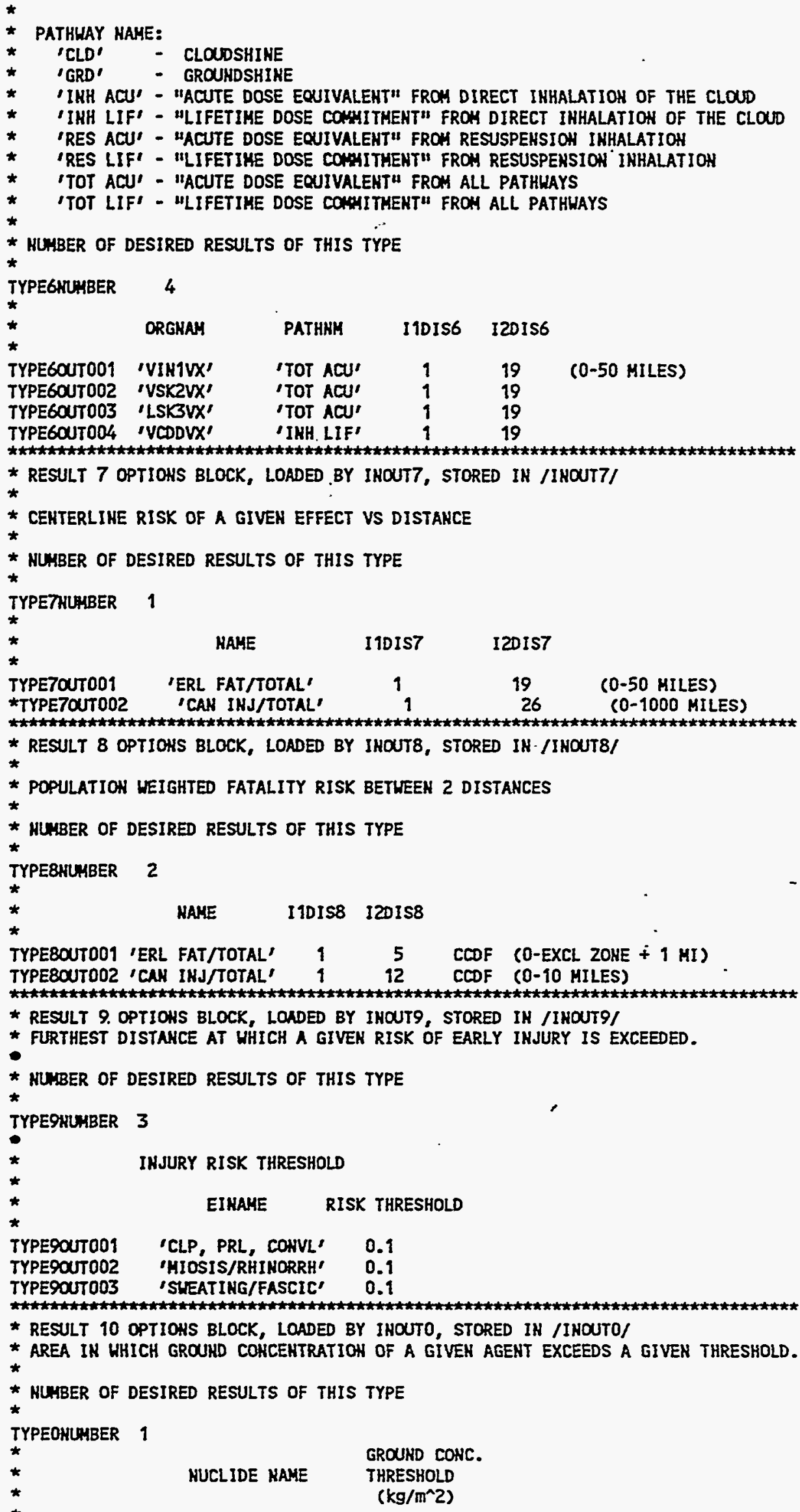


TYPEOOUTOO 'VX-LIR' 'VX-VAP' O. IE-9

\section{References}

1. C. A. Dobbe, E. R. Carison, N. H. Marshall, E. S. Marwil, and J. E. Tolli, Quality Assurance and Verification of the MACCS Code, Version 1.5, Idaho National Engineering Iaboratory, Idaho Falls, ID, NUREG/CR-5376, February 1990.

2. L. Neymotin, Comparison of MACCS Users Calculations for the International Comparison Exercise on Probabilistic Accident Consequence Assessment Codes, Brookhaven National Laboratory, Upton, NY, NUREG/CR-6053, BNL-NUREG52380, April 1994.

3. U. Tveten, Review of the Chronic Exposure Pathway Models in MACCS and Several Other Well-Known Probabilistic Risk Assessment Models, Institut for Energiteknikk, Norway, June, 1990.

4. V. L. Peterson, R. W. Patlovany, and G. A. Ennis, Comparisons Between MACCS and GENII, NSTR-017-92, EG\&G Rocky Flats, Inc., Golden, CO, October 1992.

5. U.S. Nuclear Regulatory Commission, Severe Accident Risks: An Assessment for Five U.S. Nuclear Power Plants, NUREG-1150, December 1990.

6. Lane Robbins, SAIC Approach to Verification of CHEM_MÄCCS Version $1 \mathrm{~s}$, SAF-452-95-0022, Science Application International Corp., Abingdon, MD, March 1995. 


\section{APPENDIX G}

\section{Data for Probit Equations}

The health impacts associated with various chemical agent release scenarios must be estimated for workers and members of the general population living in the vicinity. The acute health effects of greatest concern include lethality following vapor inhalation or skin contact with vapor or liquid, and nonlethal effects, such as miosis, rhinorrhea or skin irritation following vapor exposure. Chronic health effects are not addressed by the probit equations in these calculation notes.

The data used to construct the probit equations originate from the Reutter report. ${ }^{1}$ This report supplied the bliss slopes and median lethal/median effective doses for each route of exposure from which the various percentile human estimates were constructed. Dose-response probit equations for these acute and nonlethal effects are developed for exposures to inhalation and percutaneous contact for nerve agents GA, GB, VX, and the blister agent $\mathrm{HD}$. A variety of toxicologic and exposure assumptions were necessary for the construction and application of these probit equations. These assumptions include the following:

1. All vapor doses will be expressed in milligram-minutes per cubic meter; all liquid doses will be expressed as milligrams per 70-kg man.

2. Acute inhalation exposures will average $2 \mathrm{~min}$ in duration; alveolar minute ventilation for humans is assumed to be 15 liters/min.

3. Acute percutaneous vapor exposures may last up to $30 \mathrm{~min}$. -

4. Acute percutaneous liquid exposures are normalized to a 70-kg man, with body surface area of $1.8 \mathrm{~m}^{2}$.

5. The slopes of the dose-response curves for acute, nonlethal effects are comparable to those seen for the same routes of exposure for acute lethal effects.

6. Unless otherwise specified, all dose-response data are constructed for moderate temperatures (60-80 degrees Fahrenheit).

7. The vapor inhalation and percutaneous toxicity of HT is comparable to $\mathrm{HD}$.

Table G-1 summarizes the D50 and Bliss slopes for

1. Acute lethality by vapor inhalation,

2. Acute lethality by percutaneous vapor, 
3. Acute lethality by percutaneous liquid,

4. Acute non-lethal severe effects by vapor nose/eye contact,

5. Acute non-lethal mild effects by vapor nose/eye contact,

6. Acute non-lethal mild effects by vapor skin contact.

All D50 values are taken directly from Ref. 1 , which also provides the Bliss slopes for items 1 through 4.

Although Ref. 1 provides median effective doses for vapor contact with the eye, nose, and skin, resulting in miosis/rhinorrhea/sweating for nerve agents or skin/eye irritation for mustard agent, no Bliss slopes are available. In order to construct probit equations for these health effects, it is necessary to make assumptions regarding the slope of the dose-response curves for these health effects. This is done by using the Bliss slopes for acute nonlethal effects from liquid contact with skin. The risk associated with this sort of approximation is the following: If the true slopes are shallower than the severe nonlethal slopes, one may be slightly underestimating the percent of the population responding to the doses that are less than the median dose. This is probably acceptable for the risk assessment, given that these endpoints are reversible, acute mild health effects.

The data presented in Table G-1 are included in the DOSDATA.INP file, which is read by CHEM_MACCS. This DOSDATA.INP file is reproduced as Table G-2. It is possible that evaporation of deposited liquid droplets could contribute to resuspended doses because the vapor and liquid components of a given chemical are treated separately in CHEM_MACCS. Consequently, the DOSDATA.INP file contains one column for doses received directly from the plume and an adjacent column for doses due to resuspended chemicals. A zero in either column signifies that the chemical species identified on the left does not contribute to the dose in question.

Chronic health effects are not addressed by the probit equations. The potency factors associated with CDD in the DOSDATA.INP file are currently set to one. 
Table G-1. Probit constants for CHEM_MACCS

\begin{tabular}{|c|c|c|c|c|c|}
\hline Health Effect & $\begin{array}{l}\text { DOSDATA.INP } \\
\text { Dose Name }\end{array}$ & $\begin{array}{c}\text { Chemical } \\
\text { Agent }\end{array}$ & D50 & $\begin{array}{l}\text { Bliss } \\
\text { Slope }\end{array}$ & Notes \\
\hline $\begin{array}{l}\text { Acute Lethality by } \\
\text { Vapor Inhalation }\end{array}$ & VIN1 & $\begin{array}{l}\text { GA } \\
\text { GB } \\
\text { VX } \\
\text { HD }\end{array}$ & $\begin{array}{r}70 \\
35 \\
15 \\
900\end{array}$ & $\begin{array}{l}12 \\
12 \\
6.3 \\
5.7\end{array}$ & $\begin{array}{l}\text { All doses given in mg-min/m } \\
\text { Two-minute exposure duration. }\end{array}$ \\
\hline $\begin{array}{l}\text { Acute Lethality by } \\
\text { Percutaneous Vapor }\end{array}$ & VIN2 & $\begin{array}{l}\text { GA } \\
\text { GB } \\
\text { VX } \\
\text { HD }\end{array}$ & $\begin{array}{r}15,000 \\
10,000 \\
200 \\
5,000\end{array}$ & $\begin{array}{l}4.8 \\
4.8 \\
5.5 \\
6.9\end{array}$ & $\begin{array}{l}\text { All doses given in mg-min } / \mathrm{m}^{3} \text {. } \\
\text { Thity minute exposure. }\end{array}$ \\
\hline $\begin{array}{l}\text { Acute Lethality by } \\
\text { Percutaneous Liquid }\end{array}$ & LSK3 & $\begin{array}{l}\text { GA } \\
\text { GB } \\
\text { VX } \\
\text { HD }\end{array}$ & $\begin{array}{r}1,500 \\
1,700 \\
5 \\
1,400\end{array}$ & $\begin{array}{l}4.8 \\
4.8 \\
5.5 \\
6.9\end{array}$ & $\begin{array}{l}\text { All doses given in } \mathrm{mg} / 70-\mathrm{kg} \\
\text { man (body surface area }=1.8 \\
\mathrm{~m}^{2} \text { ). }\end{array}$ \\
\hline $\begin{array}{l}\text { Acute Nonlethal } \\
\text { Severe Effects by } \\
\text { Percutaneous Liquid }\end{array}$ & LSK4 & $\begin{array}{l}\text { GA } \\
\text { GB } \\
\text { VX } \\
\text { HD }\end{array}$ & $\begin{array}{l}880 \\
1,000 \\
2.5 \\
610\end{array}$ & $\begin{array}{l}4.8 \\
4.8 \\
5.5 \\
2.2\end{array}$ & $\begin{array}{l}\text { All doses given in mg/70-kg- } \\
\text { man (body surface area }=1.8 \\
\mathrm{~m}^{2} \text { ). GA, GB, and VX cause } \\
\text { collapse, paralysis, and } \\
\text { convulsions. HD causes marked } \\
\text { skin blistering and redness. }\end{array}$ \\
\hline \multirow[t]{2}{*}{$\begin{array}{l}\text { Acute Nonlethal Mild } \\
\text { Effects by Vapor } \\
\text { Nose/Eye Contact }\end{array}$} & VN5 & $\begin{array}{l}G A \\
G B \\
V X\end{array}$ & $\begin{array}{l}0.5 \\
0.5 \\
0.09\end{array}$ & $\begin{array}{l}4.8 \\
4.8 \\
5.5\end{array}$ & \multirow{2}{*}{$\begin{array}{l}\text { All doses given in mg-min/ms. } \\
\text { Two-minute exposure. GA, GB, } \\
\text { and VX cause miosis/minorthea. } \\
\text { HD causes eye irritation. Bliss } \\
\text { slopes not cited in Reference } 1 \text {, } \\
\text { taken to be same as for LSK4 } \\
\text { (see text). }\end{array}$} \\
\hline & VE5 & HD & 25 & 2.2 & \\
\hline \multirow[t]{2}{*}{$\begin{array}{l}\text { Acute Nonlethal Mild } \\
\text { Effects by Vapor Skin } \\
\text { Contact }\end{array}$} & vsw6 & $\begin{array}{l}G A \\
G B \\
V X\end{array}$ & $\begin{array}{c}2,000 \\
1,200 \\
10\end{array}$ & $\begin{array}{l}4.8 \\
4.8 \\
5.5\end{array}$ & \multirow{2}{*}{$\begin{array}{l}\text { All doses given in mg-min/m } \\
\text { Thirty-minute exposure. GA, } \\
\text { GB, and VX cause } \\
\text { sweating/fasciculation. HD } \\
\text { causes mild slin redness. Bliss } \\
\text { slopes not cited in Reference } 1 \text {, } \\
\text { taken to be same as for LSK } 4 \\
\text { (see text). }\end{array}$} \\
\hline & VSK6 & $\mathrm{HD}$ & 25 & 2.2 & \\
\hline
\end{tabular}


Table G-2. DOSDATA.INP file for CHEM_MACCS

CHEM_MACCS File DOSDATA.INP: Changed by E. HASKIN, 10APR95

Dose conversion factors for CHEM MACCS version 1.0

9 DOSES DEFINED IN THIS FILE

VINI

VSK2

ISK3

ISK4

VN5

VE5

VSW6

VSK6

VCDD

GA-VAP

8 CHEMICAL SPECIES IN THIS FILE

GB-VAP

VX-VAP

HD-VAP

GA-IIQ

GB-LIQ

VX-IIQ

HD-LIQ

VIN1

GA-VAP

$G B-V A P$

VX-VAP

HD-VAP

GA-IIQ

GB-IIQ

VX-IIQ

HD-LIQ

VSK2

GA-VAP

GB-VAP

VX-VAP.

HD-VAP

GA-IIQ

GB-IIQ

VX-IIQ

HD-IIQ

ISK3

GA-VAP

GB-VAP

VX-VAP

HD-VAP

GA-IIQ

GB-IIQ

VX-LIQ

HD-IIQ

ISK 4

GA-VAP

IPWAY

P-D50/Q R-D50/Q BLISS

GB-VAP

70
3
15
900

70

35

15

900

0

0

0

0

1

15000

10000

200

5000

0

0

0

70

12

12

$\begin{array}{rr}15 & 6.3 \\ 900 & 5.7\end{array}$

70

35

12

12

15

900

6.3

5.7

$\begin{array}{rr}15000 & 4.8 \\ 10000 & 4.8 \\ 200 & 4.8 \\ 5000 & 4.8 \\ 15000 & 4.8 \\ 10000 & 4.8 \\ 200 & 4.8 \\ 5000 & 4.8\end{array}$

$-1$

0

0

0

0

1500

1700

5

1400

$-1$

0

0
0

4.8

4.8

4.8

4.8

4.8

4.8

4.8

4.8

0

0

G-4 


\begin{tabular}{|c|c|c|c|}
\hline$V X-V A P$ & 0 & 0 & 0 \\
\hline HD-VAP & 0 & 0 & 0 \\
\hline GA-IIQ & 880 & 0 & 4.8 \\
\hline GB-LIQ & 1000 & 0 & 4.8 \\
\hline$V X-I I Q$ & 2.5 & 0 & 5.5 \\
\hline HD-IIQ & 610 & 0 & 2.2 \\
\hline VN5 & 5 & & \\
\hline GA-VAP & 0.5 & 0.5 & 4.8 \\
\hline GB-VAP & 0.5 & 0.5 & 4.8 \\
\hline VX-VAP & 0.09 & 0.09 & 5.5 \\
\hline $\mathrm{HD}-\mathrm{VAP}$ & 0 & 0 & 0 \\
\hline GA-IIQ & 0 & 0.5 & 4.8 \\
\hline GB-IIQ & 0 & 0.5 & 4.8 \\
\hline VX-IIQ & 0 & 0.09 & 5.5 \\
\hline HD-IIQ & 0 & 0 & 0 \\
\hline VE5 & 5 & & \\
\hline GA-VAP & 0 & 0 & 0 \\
\hline GB-VAP & 0 & 0 & 0 \\
\hline VX-VAP & 0 & 0 & 0 \\
\hline $\mathrm{HD}-\mathrm{VAP}$ & 25 & 25 & 2.2 \\
\hline GA-IIQ & 0 & 0 & 0 \\
\hline GB-IIQ & 0 & 0 & 0 \\
\hline VX-LIQ & 0 & 0 & 0 \\
\hline HD-LIQ & 0 & 25 & 2.2 \\
\hline VSW6 & 1 & & \\
\hline GA-VAP & 2000 & 2000 & 4.8 \\
\hline GB-VAP & 1200 & 1200 & 4.8 \\
\hline$V X-V A P$ & 10 & 10 & 5.5 \\
\hline $\mathrm{HD}-\mathrm{VAP}$ & 0 & 0 & 0 \\
\hline GA-IIQ & 0 & 2000 & 4.8 \\
\hline GB-IIQ & 0 & 1200 & 4.8 \\
\hline VX-IIQ & 0 & 10 & 5.5 \\
\hline HD-IIQ & 0 & 0 & 0 \\
\hline VSK6 & 1 & & \\
\hline GA-VAP & 0 & 0 & 0 \\
\hline GB-VAP & 0 & 0 & 0 \\
\hline$V X-V A P$ & 0 & 0 & 0 \\
\hline $\mathrm{HD}-\mathrm{VAP}$ & 50 & 50 & 0 \\
\hline GA-IIQ & 0 & 0 & 0 \\
\hline GB-IIQ & 0 & 0 & 0 \\
\hline VX-IIQ & 0 & 0 & .0 \\
\hline HD-IIQ & 0 & 50 & 2.2 \\
\hline $\mathrm{VCDD}$ & 6 & & \\
\hline GA-VAP & 1 & 1 & 0 \\
\hline GB-VAP & 1 & $\overline{1}$ & 0 \\
\hline$V X-V A P$ & 1 & 1 & 0 \\
\hline $\mathrm{HD}-\mathrm{VAP}$ & 1 & 1 & 0 \\
\hline GA-IIQ & 0 & 1 & 0 \\
\hline GB-IIQ & 0 & 1 & 0 \\
\hline VX-LIQ & 0 & 1 & 0 \\
\hline $\mathrm{HD}-\mathrm{IIQ}$ & 0 & 1 & 0 \\
\hline
\end{tabular}


Reference for Appendix G

1. Sharon Reutter, et. a1., Review of the Available Data and Existing Human Toxicity Estimates for Selected Chemical Agents and Human Toxicity Estimates for Appropriate for the Protection of the Soldier, U.S. Army Research, Development, and Engineering Center Report of CDE-PAT Toxicology Subgroup, In Press. 


\section{CHEM_MACCS DISTRIBUTION LIST}

Christiana Lui

T10E50

RES/DST/PRAB

U.S. Nuclear Regulatory Commission

Washington, DC 20555

Mr. Steve Fisher

MS-8057

Oak Ridge National Laboratory

P.O. Box 2009

Oak Ridge, TN 37831

Mr. Vern Peterson

EG\&G Rocky Flats, Inc.

Rocky Flats Plant

P.O. Box 464

Bldg. T886B

Golden, CO 80402-0464

Mr. Joe Shinn

Mailstop L-453

Lawrence Livermore National Laboratory

P.O. Box 808

Livermore, CA $94551-9900$

Mr. Anthony Savino

Westinghouse Hanford Company

P.O. Box 1970 (MSIN H4-64)

2355 Stevens Drive

Richland, WA 99352

Prof. Eric Haskin

Department of Chemical and Nuclear Eng.

Farris Engineering Center

University of New Mexico

Albuquerque, NM 87131-1341

Louis Restrepo

Organization 9364

Sandia National Laboratories

P.O. Box 5800

Albuquerque, NM 87185-1175

Dr. Kamiar Jamali

Office of Engineering and Operations Support

DP-62/GTN

Department of Energy

Washington, DC 20585

David Chanin

3209 Campus Blvd., NE

Albuquerque, NM 87106
Roy Karimi

SAIC

20201 Century Blvd

3rd Floor

Germantown, Maryland 20874

Dae Chung

Office of Engineering \& Operations

DP-62/GTN

Department of Energy

Washington, D.C. 20585

Marcia Middleton

SAIC

9 Aberdeen Shopping Plaza

Aberdeen, MD 21001

Gary Boyd

SAIC

1309 Continental Dr.

Suite $F$

Abingdon, MD 21009

Douglas Woody

SAIC

1309 Continental Dr.

Suite F

Abingdon, MD 21009

Rob Bailey

SAIC

1309 Continental Dr.

Suite F

Abingdon, MD 21009

Chuanyi Ding

Chemical \& Nuclear Engineering

Farris Engineering Center

University of New Mexico

Albuquerque, NM 87131

Kenneth J. Summa

Chemical \& Nuclear Engineering

Farris Engineering Center

University of New Mexico

Albuquerque, NM 87131 


\section{CHEM_MACCS DISTRIBUTION LIST}

INTERNAL DISTRIBUTION

MS0748 Mary L. Young, 6413

MS9018 Central Technical Files, 8523-2 (1)

MS0899 Technical Library, 4414 (5)

MS0619 Review \& Approval Desk, 12630

For DOE/OSTI

(2) 